10.90 .818 $30 \%=0$ $0 \times 2 \% 07: 000 \%$ Tof 200 6t $y=0.00$ D. $9: 0 \% 0$ - $2 \%$. 0.0 $\therefore \quad(.3-9)$

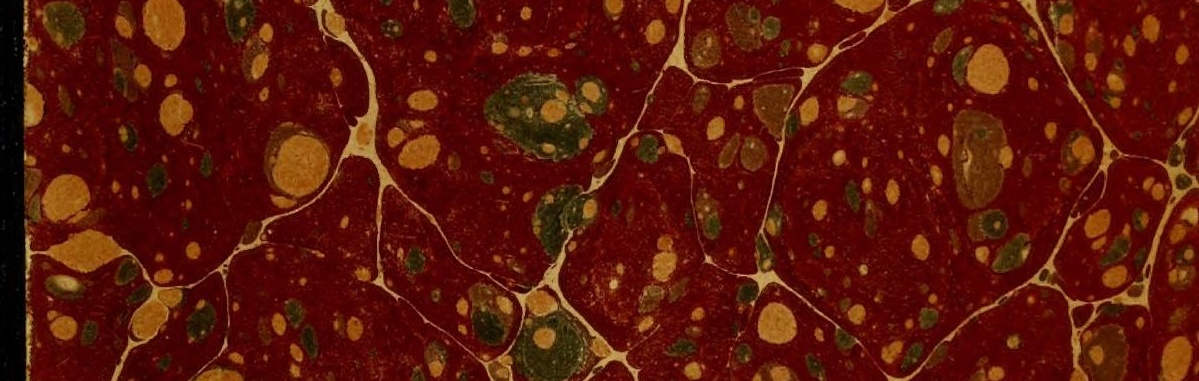
$-400.0 \div 0$ $610+000 \%$

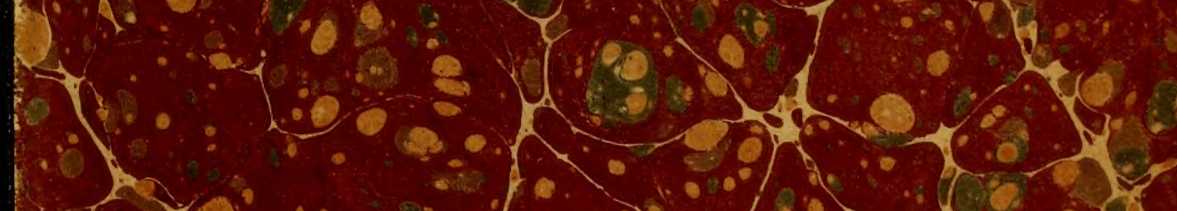
$\left(\begin{array}{cc}0 \\ 0\end{array}\right.$ 


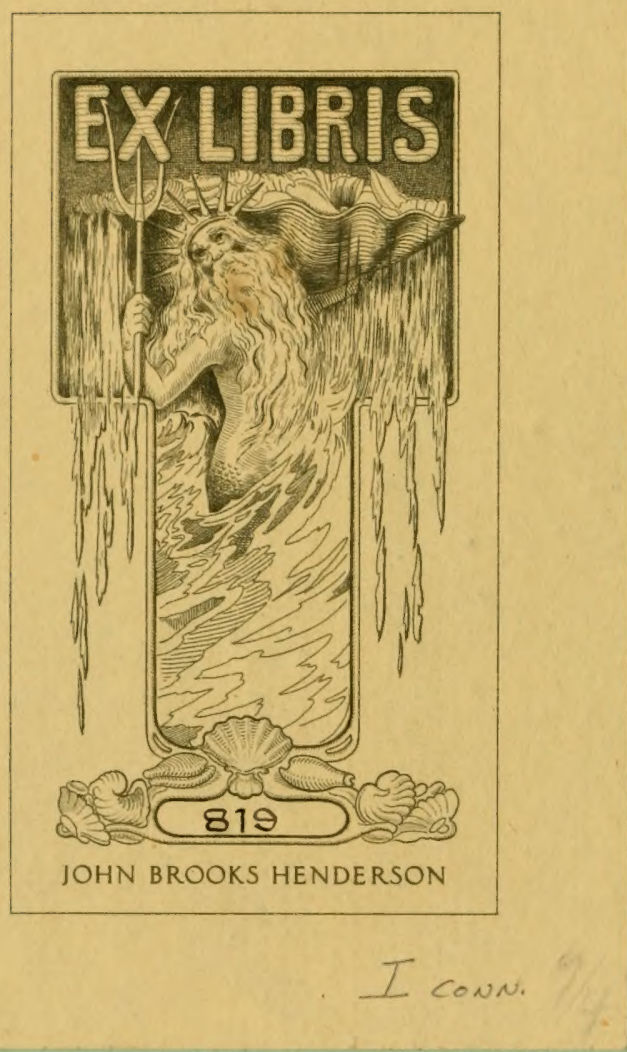


Devision of Molluskses

Seotianal Library

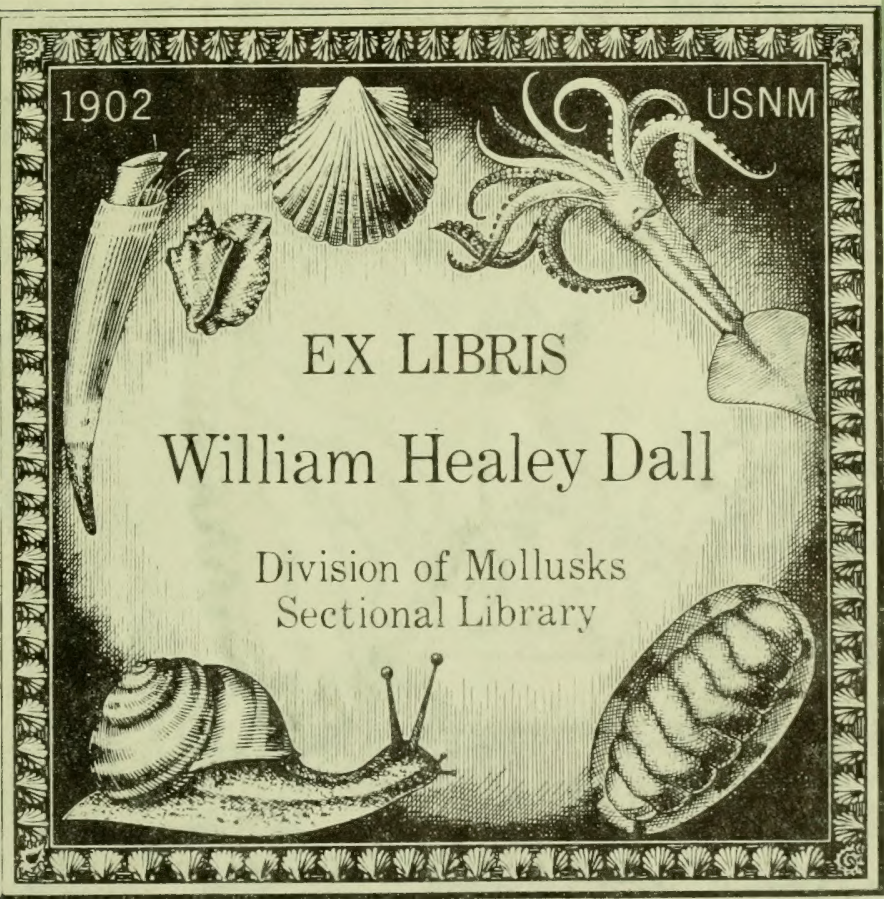







\title{
ANNALS
}

OF THE

\section{SOUTH AFRICAN MUSEUM}

\author{
VOLUME XI.
}

PART III. containing:-

3. - A Revised Reference List of South African Non-marine Mollusca; with Descriptions of New Species in the South African Museum. By M. Connoluy (Plate II.)

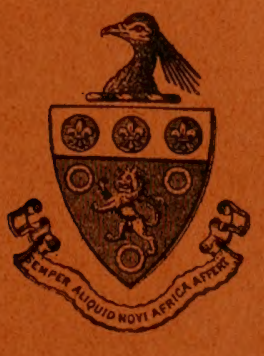

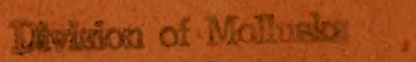

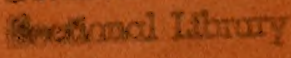

ISSUED OCTOBER 24th, 1912. PRICE 12:

PRINTED FOR THE

TRUSTEES OF THE SOUTH AFRICAN MUSEUM By West, Newman \& Co., London. 



\section{3.-A Revised Reference List of South African Non-marine Mollusca; with Descriptions of New Species in the South African Museum.-By M. Connoluy.}

\section{INTRODUCTION.}

LitTLE more than thirteen years have elapsed since the publication of Messrs. Melvill and Ponsonby's "Contribution towards a CheckList of the Non-marine Molluscan Fauna of South Africa" (Proc. Mal. Soc., 1898, iii., p. 166-184), which included all species then known south of the Tropic of Capricorn; and of Dr. Sturany's "Catalog der . . südafrikanischen Land-und Süsswasser-Mollusken" (Wien, 1898), in which he described several new forms, and listed, with few exceptions, all older ones which had been up to that date reported from south of the line roughly formed by the Zambesi and Kunene Rivers.

In this short time, however, the number of non-marine shells known in South Africa has increased by nearly one-half, the 408 species listed by Sturany having grown to 569, despite the fact that many forms then considered distinct have since been altogether expunged or placed in synonymy.

No apology, therefore, is needed for the appearance of a revised reference list, and it only remains to add a few words of explanation as to special features introduced in the new work.

At Dr. Péringuey's desire, I have adopted Sturany's more extended geographical boundaries. Although Messrs. Melvill and Ponsonby's invaluable series of articles in the Ann. and Mag. Nat. History has left comparatively little to be cleared up regarding the non-marine mollusca to the south of the Tropic of Capricorn, very little is known, conchologically, of much of the region just north of that limit, and the present list must necessarily leave much room for amplification in this direction.

Very few South African non-marine fossils are yet known, and most of them were described in papers dealing also with recent shells. I have therefore included both recent and fossil species in 
the body of my work, but, to facilitate distinction, the reference number is printed in ordinary type before recent species, and in itatics before those which have only been found, so far, in a fossil condition in South Africa.

A list of authors and works consulted, with the abbreviations used, is given on p. 288. Owing to the great increase of recent literature on the subject, the list of references under each species has been lightened by the omission of all those of a purely "check-list" character, or of no actual value to the student.

Many comparatively unimportant references to classification or locality only are also omitted, the authority quoted after each locality being, in the latter case, considered sufficient.

I have also omitted many of the older references to European and American species, as the descriptions contained in them are of little value.

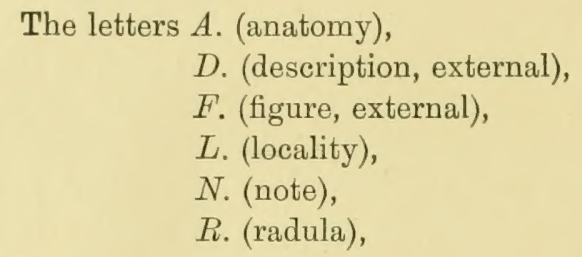

are appended respectively to each reference to denote the class of information it contains.

With regard to Férussac's Tabl. Syst. des Moll., part iii., of which there were two editions in the same year, with different pagination, the alternative pages are given.

The species described and figured by Sturany in Südafr. L.- und S.-W.-Moll., November, 1898, were first published by him in a "Preliminary Note" in the Anz. kais. Akad. Wiss. Wien, in June of the same year. I have not, in this instance, quoted the earlier reference, except in the case of Chondrocychus isipingöensis, where priority of nomenclature is involved.

The present work is intended, primarily, to be of as much use as possible to students in South Africa. This accounts for my having appended full references to Genera and the higher divisions, many of which I have found it quite impossible to trace in any library in that country; and also for the addition of as many localities for each species as I have been able to gather; but it has been, naturally, quite impossible to verify the correctness of all of the latter. Many of the older specific names have been misapplied, at various times, to other species, while many recently described shells 
have wrongly figured, prior to their description, under one of the older names. In giving all these localities, however, it is my hope that future collectors, on finding a certain shell in a certain neighbourhood, will at least have some guide as to the known species with which their specimens may be compared.

No single map is yet published which includes all the localities mentioned, but to simplify matters as far as possible I have given the province in which each is situated, and subdivided the larger or more intricate districts. Thus, German South-West Africa is divided into Ovampoland (north), Damaraland (or Hereroland) (central), and Great Namaland (south). Little Namaland is in the Cape of Good Hope Province, just south of the mouth of the Orange, and Griqualand West is that portion of the colony north of the same river. Cape Peninsula, where specially mentioned, refers to the small, but conchologically rich, district between Cape Town and Cape Point.

British Bechuanaland, on the north, is divided from Bechuanaland by a line nearly following the direction of the Molopo River. Lorenzo Marques represents the whole Portuguese territory in East Africa south of the Zambesi, as distinct from Mozambique to its north ; while the Northern Transvaal comprises the district north of Pretoria.

An effort has been made to state where the type of each species is preserved, and through the kindness of the custodians of the collections mentioned, I have been able to locate about nine-tenths of the total number. In cases where the type cannot be traced or was never specified, the whereabouts of original specimens has, when possible, been given.

For the information of local students, the letters S.A.M. are affixed to the names of species contained in the collection of the South African Museum. I may add that the magnificent Burnup Collection is exhibited in the Natal Government Museum, Pietermaritzburg, while there are good collections of non-marine mollusca at Grahamstown and Port Elizabeth, and a smaller one at Kimberley.

It only remains for me to express my most grateful thanks to the many kind friends who have been ever ready to assist me in the many difficulties which have cropped up in the course of even the present unassuming work. It is almost impossible to mention all by name, and I must therefore confine myself on this page to acknowledging my extreme indebtedness to Messrs. J. H. Ponsonby and H. C. Burnup for an enormous amount of invaluable help and advice, to which are due, in great measure, any merits which may eventually be found in the ensuing pages. 
The arrangement of Families and Genera is in accordance with the latest views of leading authorities on the subject. That of the higher orders is a slightly modified adaptation of Paul Pelseneer's classification of the Mollusca in Part V. of Sir E. Ray Lankester's "Treatise on Zoology." (London, 1906.)

In the use of Family names, I have followed those writers who employ a few only, in a wide sense, rather than a larger and possibly unnecessary number of smaller families.

April, 1912.

\section{REFERENCE LIS'I.}

\section{Sub-Kingdoni IMOLLUSCA, Cuvier, 1795.}

(La Décade Philos. Litt. et Politique, Paris, v. p. 391, as Mollusques.)

Giade PRORHIPIDOGLOSSOMORPHA, Grobben, 1894.

(Sitz.-Ber. Akad. Wiss. Wien, p. 86.)

Chass GASTROPODA, Cuvier, 1795.

(Magasin Encycl, ii. p. 448, as Gastéropodes.)

Sub-Class EUTHYNEURA, Spengel, 1881.

(Zeitschr. f. wissens, Zool. Leipzic, p. 372.)

Order PULMonata, Cuvier, 1817.

(Règne Anim. ii. pp. 387, 401, as Pulmonés.)

Sub-Order STYLOMMATOPHORA, Schmidt, 1855.

(Abhandl. Naturwiss, vereins. Sachs. Halle, p. 7.)

'Tribe AGNATHA, Mörch, 1859.

(Mal. Blätt. vi. p. 109.)

FAMiLy APERIDE, Möllendorff, 1903.

(Conch. Cab. p. 5.)

Genus APERA, Heynemann, 1885.

(Jahrb. d. Deutsch. Mal. Ges. xii. p. 20.)

(=Chlamydephorus, Binney, 1879, not Chlamydophorus, Harlan, in Mammalia 1825.)

Type of Genus A. gibbonsi (Binn.)

1. Apera burnupi, Smith.

1892 Apera bumupi, Smith, A.M.N.H.x. p. $466 . \quad D$. 1897

Cllge., A.M.N.H. xx. p. 221. pl. 5, f. 1-6. $A$. 
1898 Apera bumupi, Smith, Pilsb., Nautilus, xii. p. $12 . \quad N$.

1900 " " Cllge., Ann. S.A. Mus. ii. p. 4. pl. 1, f. $5,6 . N \cdot F$.

1901 " " $\quad$ Cllge., Journ, of Mal. viii. p. 71, f. 1. A. $1910 \quad$ ", $\quad$ " Cllge., Ann. Natal Mus. ii. p. 166. D.

Type in British Museum.

Hab. Natal. Pietermaritzburg (Burnup).

\section{Apera gibbonsi (Binney).}

1879 Chlamydephorus gibbonsi, Binn., Bull. Mus. Comp. Zool. Harvard, v. p. 331. pl. 2, f. $a, b$. D.F.

1884 Chlamydephorus gibbonsi, Binn., Ann. N.Y. Acad. Sci. iii. p. 81. pl. 17, f. A. Ii.

1884 Chlamydophoms gibbonsi, Binn., Tryon, Struct. and Syst. Conch. iii. p. 13. pl. 101, f. 47. D.F.

1885 Chlamydephoms gibbonsi, Binn., Tryon, Man. of Conch. i. pp. 17, 251. pl. 2, f. 95. D.F.

1885 Apera gibbonsi, Binn., Heynem., Jahrb. d. Deutsch. Mal. Ges. xii. p. 17. pl. 2, f, 5-7. D.F.

1890 Chlamydephoms gibbonsi, Binn., Ckll., A.M.N.H. vi. p. 390. D. 1900 Apera gibbonsi, Binn., Cllge., Ann. S.A. Mus. ii. p. 4. N. $1910 \quad$ " " Cllge., Ann. Natal Mus. ii. p. 165. D. Type in Academy of Natural Sciences, Philadelphia.

Hab. Natal. Umgeni Valley (Gibbons). Equeefa ; Port Shepstone; Thornybush, near Pietermaritzburg (Burnup).

Cape of Good Hope (IVeale).

\section{Apera natalensis, Collinge.}

1900 Apera natalensis, Cllge., Ann. S.A. Mus. ii. p. 3. pl. 1, f. 3, 4. pl. 2 , f. 14, 15. D.F.A.

1910 Ann. Natal Mus. ii. p. 167. D.

Type in South African Museum.

Hab. Natal. Richmond (Ward).

4. Apera purcelti, Collinge.

1901 Apera purcelli, Cllge., Ann. S.A. Mus. ii. p. 230. pl. 14, f. 1, 2. D.F.

Type in South African Museum.

Hab. Cape Peninsula. Table Mountain (Lightfoot). 
FAMILY TESTACELLID ZE, Gray, 1853.

(A.M.N.H. xii. p. 330.)

Genus TESTACELLA, Cuvier, 1800.

(Leç. d'Anat. comp. Paris, i. Table 5.)

Type of Genus T. haliotidea, Drap.

5. Testacelda aurigaster, Layard.

1893 Testacella aurigaster, Lay., Ckll., Conchologist, ii. p. 205. N. 1902 ", (as Syn. of T. mangei, Fér.) Tayl., Mon. Brit. Moll. pp. 25, 27. L.

Hab. Cape Peningula. Cape Town (Layard; Lightfoot).

I believe I am correct in stating that Layard actually published the description of this species, but in what periodical cannot be traced. It is nearly related to $T$. mangei, Fér., with which it may probably prove to be synonymous.

Family STREPTAXID无, Gray, 1860.

(A.M.N.H. vi. p. 268.)

Sub-Family STREPTAXINÆ, Godwin-Austen, 1908.

(Fauna Brit. India, Moll. p. 1.)

Genus STREPTAXIS, Gray, 1837.

(A.M.N.H. i. p. 484.)

Type of Genus, S. nobilis, Gray.

6. Streptaxis gwandaensis, Preston.

1912 Streptaxis gwandaensis, Prest., A.M.N.H. ix. p. 69, f. 1. D.F. Type in coll. Preston.

Hab. Rhodesia. Near Geelong Mine, Gwanda District (S. B. Cox).

Sub-Family ENNEIN Æe, Kobelt, 1904.

(Conch. Cab. p. 92.)

Genus ENNEA, H. and A. Adams, 1855.

(Gen. Rec. Moll. ii. p. 171.)

Type of Genus, E. elegantula (Pfr.).

The South African portion of this Genus is undoubtedly in need of severe critical revision. Many species have been differentiated on 
very slight peculiarities of form, sculpture, or dentition. However, in the recently described $E$. johannesburgensis, $M$. and $\mathrm{P}$., it will be found that, while the form and sculpture change but little, the presence or complete absence of the basal tooth, even among shells from under the same stone, is a matter of indifference; while in E. xysila, M. and P., though the dentition remains practically unchanged, the length of full-grown specimens varies from 5 to $8 \mathrm{~mm}$, and the sculpture is sometimes almost lirate, sometimes almost invisible, quite irrespective of the size of the shell.

It can be easily understood, therefore, that, in more widely distributed species, the range of variation increases with the extended geographical distribution; but in many such cases intermediate varieties are found in intermediate localities, which link up a complete chain of connection between comparative extremes of form.

Taking these facts into consideration, it is highly probable that many species at present reckoned distinct will, on fuller comparison, have to be referred to merely varietal rank.

As no satisfactory method of subdividing the South African Enneinæ has yet been contrived, the subjoined list of species is given in alphabetical order, omitting all reference to any subgenera or sections hitherto proposed.

7. Ennea adamsiana, Pfeiffer.

1859 Ennea adamsiana, Pfr., Novit. Conch. i.p. 114.pl.32, f.9-11. D.F. 1859

$1885 \quad$ " "Tryon, Man. of Conch. i. p. 98. pl. 19, " Mon. Hel. iv. p. 339. D. f. $85 . D$ D.F.

$1904 \quad$, $\quad$, Kob., Conch. Cab. p. 192.pl.24, f.8. D.F.

Type in British Museum.

Hab. Natal. Port Natal (in coll. Pfeiffer). Widely distributed.

Cape of Good Hope. "Said to be found at Korsten, Port Elizabeth" (Crawford).

var. impervia, Melv. \& Pons.

1896 Ennea impervia, M. \& P., A.M.N.H. xviii. p. 315. pl. 16,f. 1. D.F' 1904 Kob., Conch.Cab.p.233.pl.32,f.21. D.F.

Type in British Museum.

Hab. Nata (fide M. \& P.). Lower Umkomaas; Equeefa ; Port Shepstone (Burnup).

APE OF Good Hope. Port St. John's (Shortridge). 
It appears inadvisable to regard impervia as other than a wellmarked variety of adamsiana, being a stouter form with magnified dentition. Impervia is found generally along the coast-belt of Natal, with a very small sprinkling of the narrower form. At Pine Town it is difficult to differentiate the two; at Botha's Hill they are enigmatical ; while on reaching Pietermaritzburg and the surrounding district only the narro:ver form is found.

8. ENnea albersi (Pfeitfer).

1854 Pupa albersi, Pfr., P.Z.S. p. 295. D.

1855 Ennea albersi, Pfr., Mal. Blätt. ii. p. 61. D.

\begin{tabular}{|c|c|c|c|}
\hline 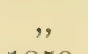 & $"$ & $"$ & Novit. Conch. i. p. 38. pl. 10, f. 15-17. D.F. \\
\hline 1859 &, & , & Mon. Hel. iv. p. $338 . \quad D$. \\
\hline 1885 & $"$, & $"$ & $\begin{array}{l}\text { Tryon, Man. of Conch. i. p. 97. pl. 19, f. 83, } \\
\text { 84. D.F. }\end{array}$ \\
\hline 190 & "' & "' & M. \& P., A.M.N.H. vii. p. 596 . N.F. \\
\hline & " & & Jonch. C \\
\hline
\end{tabular}

Type in British Museum.

Hab. Natal. Port Natal (Strangier, fide Pfeiffer 1859). Port Shepstone, both sides of the River Umzimkulu (Burnup).

9. Ennea alicie, Melv。 \& Pons.

1907 Ennea alicia, M. \& P., A.M.N.H. xix. p. 95. pl. 6, f. 1. D.F. Type in British Museum.

Hab. Zululind. Makowe (Burnup). Dukuduku Forest (Toppin).

This may possibly be conspecific with $E$. dadalea, M. and P. Size is the chief distinguishing characteristic, and intermediate grades exist.

10. Ennea aperostoma, Melv. \& Pons.

1892 Enneacaperostoma,M.\&P., A.M.N.H. ix. p. 93. pl. 6, f. 10. D.F. 1904 Kob.,Conch.Cab.p.219.pl.27,£.10.D.F.

Type in British Museum.

Hab. Natal (Layard).

var. Lissophanes, Melv. \& Pons.

1892 Ennea aperostoma var. lissophanes, M. \& P., A.M.N.H. ix. p. $93 . N$.

Type in British Museum.

Hab. Natal (Layard). 
11. Ennea arnoldi, Sturany.

[S.A.M.]

1898 Ennea amoldi, Stur., S. A. Moll. p. 28. pl. 2, f. 26-30. D.F. $1904 \quad$ " $\quad$ Kob.,Conch.Cab.p.203.pl.25,f.10-13. D.F. Type in Naturh. Hofmus. Vienna.

Hab. Natal. Isipingo; Durban (Penther). Tongaat; Lower Umkomaas (Burnup).

\section{var. ELONGATA, Sturany.}

1898 Ennea annoldi var. elongata, Stur., S. A. Moll. p. 29. pl. 2, f. 31 . D.F.

Type in Naturh. Hofmus. Vienna.

Hab. Natal. Isipingo (Penther).

12. Ennea auris-leporis, Melv. \& Pons.

1898 Ennea auris-leporis, M. \& P., A.M.N.H. i.p. 25. pl. 8, f. 3. D.F. $1904 \quad$ ", "Kob., Conch. Cab. p. 182. pl. 23, f. 10 . D.F.

Type in British Museum.

Hab. Natal (fide M. and P.).

\section{Ennea Berthe, Melv. \& Pons.}

1901 Ennea berthe, M. \& P., A.M.N.H. i. p. 315. pl. 2, f. 1. D.F. $1904 \quad$ " $\quad$ Kob., Conch. Cab.p. 235.pl.32,f.20. D.F.

Type in British Museum.

Hab. Natal. Karkloof Bush (McBean). Ntimbankulu (Burnup).

14. Ennea bowkere, Melv. \& Pons.

1892 Ennea bowkerce, M. \& P., A.M.N.H. ix. p. 92. pl. 6, f. 9. D.F. $1904 \quad$ " $\quad$, Kob., Conch.Cab.p.219.pl.27,f.9. D.F.

Type in British Museum.

Hab. Cape of Goon Hope. East London (Miss Bowker).

15. Ennea burnupi, Melv. \& Pons.

1897. Ennea burnupi, M. \& P., A.M.N.H. xix. p. 634. pl.17, f.2. D.F. $1904 \quad$ " $\quad$ Kob., Conch.Cab.p.217.pl.27, f.5. D.F.

Type in British Museum.

Hab. Natal. Town Bush, Pietermaritzburg; Gordon Falls (Burnup). 
16. Ennea cairnsi, Melv. \& Pons.

1897 Ennea caimsi, M. \& P., A.M.N.H. xix. p. 634. pl. 17, f. 1. D.F. $1904 \quad$ " $\quad$ Kob., Conch. Cab.p.216.pl.27, f.4. D.F.

Type in British Museum.

Hab. Cape of Good Hope. Mouth of Buffalo River (in coll. Cairns ; Burnup).

17. Ennea cathista, Melv. \& Pons.

1909 Ennea callista, M. \& P., A.M.N.H.iv. p. 485. pl. 8, f. 1. D.F.

Type in British Museum.

Hab. Cape of Good Hope. Dassie Krantz, Grahamstown (Farquhar).

18. Ennea calopasa, Melv. \& Pons.

1903 Ennea calopasa, M. \& P., A.M.N.H. xii. p. 596. D.F.

Type in British Museum.

Hab. Natal. Port Shepstone, both sides of the River Umzinkulu (Burnup).

19. Ennea candidula, Morelet.

1889 Ennea candidula, Morel., J. de C. xxxvii.p. 12. pl. 2, f. 2. D.F. 1890 " layardi, Ancey, Bull. Soc. Mal. Fr, vii. p. 159. D.

1904 " , " , Kob., Conch. Cab. p. 242. D.

" " candidula, Morel., Kob., Conch. Cab. p. 240. pl. 29,

Type in British Museum.

f. 6-8. D.F.

Hab. Cape of Good Hope. Port Elizabeth, South End, and Kragga Kama, 17 miles south of Port Elizabeth (Crawford). Port Elizabeth and Grahamstown (layardi, fide Layard).

NataL. Upper Tugela (Quekett).

20. Ennea caryatis, Melv. \& Pons.

1898 Ennea caryatis, M. \& P., A.M.N.H. i. p. 24. pl. 8, f. 2. D.F. $1904 \quad$ " $\quad$ Kob.,Conch.Cab.p.134.pl.19,f.13. D.F'.

Type in British Museum.

Hab. Cape of Good Hope. Cradock (Farquhar). Prieska (Gibbons; Gould).

21. Ennea cimolia, Melv. \& Pons.

1895 Ennea cimolia, M. \& P., A.M.N.H. xvi.p. 478. pl. 18, f. 2. D.F. 1904 Kob., Conch. Cab. p. 185. pl. 23, f. 15. D.F. 
Type in British Museum.

Hab. Cape of Good Hope. Grahamstown (Farquhar). Pirie Forest (var., Godfrey).

Natal. Dargle; Nottingham Road; Karkloof; Edendale; Backworth and Ntimbankulu, Mid-Illovo (Burnup).

$E$. pentheri, Sturany, though much smaller, seems otherwise identical.

22. Ennea cionis, Melv. and Pons.

1898 Ennea cionis, M. \& P., A.M.N.H. i. p. 25. pl. 8, f. 4. D.F.

$1904 \quad$ " $\quad$ Kob., Conch. Cab.p. 135̄.pl. 19, f. 15. D.F.

Type in British Museum.

Hab. Cape of Good Hope. Van Staadens River, Port Elizabeth (Crawford).

Zululand. Melmoth (Miss Hickey).

23. Ennea claustraria, Melv. \& Pons.

1903 Ennea claustraria, M. \& P., A.M.N.H. xii.p. 597.pl. 31,f.16. D.F. 1907 "stauroma, " " $\quad$ xix. p. 96. pl.6,f.4. D.F.

Both types in British Museum.

Hab. Zululand. Lower Umfolosi Drift (Burnup). Melmoth (stauroma, Miss Hickey).

Almost identical with infrendens, von Mts., except that the latter has a small plait on the columellar lip, just above the basal tooth, where claustraria has only a slight swelling.

24. Ennea columnella, Melv. \& Pons.

1901 Ennea columnella, M. \& P., A.M.N.H. viii. p. 316. pl. 2, f. 2. D.F. $1904 \quad$ " " Kob.,Conch.Cab.p.235.pl.32,f.22.D.F.

Type in British Museum.

Hab. Natal. Karkloof Bush (McBean). Dargle; Edendale; Ntimbankulu (Burnup).

25. Ennea connoluyt, Melv. \& Pons.

1909 Ennea connollyi, M. \& P., A.M.N.H.iv. p. 486. pl. 8, f. 2. D.F. Type in British Museum.

Hab. Natal, Majuba (Connolly).

Transvaal. Hennop's River; Buis Kop (Connolly).

Generally a much narrower shell than the figure would suggest. 
26. Ennea consobrina, Ancey.

1892 Ennea consobrina, Ancey, Brit. Nat. p. 125. D.

1898

1904

,

" M.\&P., A.M.N.H.i.p.24.pl.8,f.9. N.F.

" Kob., Conch. Cab. pp. 232, 289. pl. 28, f. 19 . D.F.

Type in coll. Tomlin.

Hab. Cape of Good Hope. Albany (Miss Glanville).

27. Ennea crassidens, Pfeiffer.

1859 Ennea crassidens, Pfr., Novit. Conch. i. p. 114. pl. 32, f. 6-8. D.F. " " " Mon. Hel. iv. p. 340 . D.

1885 " „ Tryon, Man. of Conch. i. p. 97. pl. 19, f. $100 . D . F$.

1898 Ennea durbanensis, Stur., S. A. Moll. p. 25. pl. 1, f. 17. D.F. 1904 " " Kob., Conch. Cab. p. 203. pl. 25, f. 14 . D.F.

" Ennea crassidens, Pfr., Kob., Conch. Cab. p. 192. pl. 24, f. 7. D.F.

Type of crassidens in British Museum; durbanensis in Naturb. Hofmus. Vienna.

Hab. Natal. Port Natal (Plant). Durban Bluff; Isipingo (durbanensis, Penther). Pinetown; Equeefa (Burnup).

28. Ennea crassilabris, Craven.

1880 Ennea crassilabris, Crvn., P.Z.S. p. 616. pl. 57, f. 5. D.F.

1885 , " Tryon, Man. of Conch. i. p. 102. pl. 20, f. 50, 51. D.F.

$1904 \quad$, " Kob., Conch. Cab. p. 136. pl. 19, f. $16 . \quad D . F$.

1909 Ennea enschemon, M. \& P., A.M.N.H. iv. p. 487. pl.8, f. 5. D.F.

Both types in British Museum.

Hab. Transvaal. Lydenburg (crassilabris, Craven). Pietpotgietersrust; Pruizen (euschemon, Connolly).

The type of euschemon is rather smaller than that of crassilabris, but is not specifically distinct.

29. Ennea craterodon, Melv. \& Pons.

1903 Ennea craterodon, M. \& P.,A.M.N.H. xii. p. 597. pl. 31, f. 9. D.F. Type in British Museum.

Hab. Cape of Good Hope. Maestrom Forest, Bedford (Farquhar). 
30. Ennea crawfordi, Melv. \& Pons.

1898 Ennea craufordi, M. \& P., A.M.N.H. i. p. 26. pl. 8, f. 5. D.F. 1904

Kob., Conch. Cab. p. 135. pl. 19,

Type in British Museum.

$$
\text { f. } 17 . D . F \text {. }
$$

Hab. Cape of Good Hope. Mouth of Van Staadens River, Port Elizabeth (Crawford).

31. Ennea crispula, Melv. \& Pons.

1909 Ennea crispula, M. \& P., A.M.N.H. iv. p. 486. pl. 8, f. 3. D.F. Type in British Museum.

Hab. Cape of Good Hope. Grahamstown (per Fulton).

32. Ennea crossleyana, Melv. \& Pons.

1893 Ennea crossleyana, M. \& P., A.M.N.H. xii.p. 106. pl. 3, f. 8. D.F. 1904

" Kob., Conch. Cab. p. 196. pl. 24,

Type in British Museum.

$$
\text { f. } 16 \text {. D. } F^{\prime} \text {. }
$$

Hab. Natal, Pietermaritzburg (Mrs. Crossley; Burnup).

A small inland form of the coastal $E$. gouldi, Pfr. Size is almost the only distinction, and as intermediate degrees are known from intermediate localities-Pinetown; Botha's Hill; Table Mountain (Burnup) -it is probable that the two species may eventually prove to be identical.

33. Ennea dedalea, Melv. \& Pons.

1903 Ennea dedalea, M. \& P., A.M.N.H. xii. p. 598, pl, 31, f. 12. D.F.

Type in British Museum.

Hab. Zululand. Lower Umfolosi Drift (Burnup).

Ennea aticia, M. \& P., is very nearly allied to this species.

34. Ennea dargleñsis, Melv. \& Pons.

[S.A.M.]

1908 Ennea darglensis, M. \& P., A.M.N.H. i. p. 130. pl. 7, f. 1. D.F. Type in British Museum.

Hab. NataL. Dargle; Richmond; Inhluzani Mountain (Burnup). Karkloof ; Nottingham Road (Taynton).

Cape of Good Hope. Gowies Kloof, Grahamstown (Farquhar).

35. Ennea delicatula, Pfeiffer.

1856 Ennea delicatula, Pfr., Mal. Blätt. iii. p. 259. D.

1859

"Novit. Conch. i. p. 117. pl. 32, f. 21-23. D.F. 
1859 Ennea deticatula, Pfr., Mon. Hel. iv. p. 340. D.

1885 ", "Tryon, Man. of Conch. i. p. 96. pl. 19, f. 2. D.F.

$1904 \quad$ ", " Kob., Conch. Cab. p. 190.pl.24, f.5. D.F.

Original, probably type, in British Museum.

Hab. Natal. Port Natal (Plant).

36. Ennea distincta, Melv. \& Pons.

1893 Ennea distincta, M. \& P., A.M.N.H. xi. p. 22. pl. 3, f. 10. D.F. 1904 „ Kob., Conch. Cab. p. 187. pl. 23, f. 19 . D.F.

Type in British Museum.

Hab. Transvan. Middelburg (Crawford).

37. Ennea docimasta, Melv. \& Pons.

[S.A.M.]

1898 Ennea dokimasta, M. \& P., A.M.N.H. i. p. 27. pl. 8, f. 7. D.F. 1898. " docimasta, " Proc. Mal. Soc. iii. p. 167. (Emend. Nom.)

1904 " dokimasta, " Kob., Conch. Cab. p. 183. pl. 23, f. 11 . D.F.

Type in British Museum,

Hab. Natal (MeKen, per Layard).

38. Ennea dolichoskia, Melv. \& Pons.

1892 Ennea dolichoskia, M. \& P., A.M.N.H.ix. p. 86. pl. 6, f. 6. D.F. 1904 " Kob., Conch. Cab. p. 218. pl. 27, f. $7 . D . F$.

Type in British Museum.

Hab. CAPE of Good Hope. Near Port Elizabeth (Langley). Mouth of Van Staadens River (Crawford).

39. Ennea drakensbergensis, Melv. \& Pons.

1893 Ennea drakensbergensis, M. \& P., A.M.N.H. xii. p. 107. pl. 3, f. 9. D.F.

1904 Kob., Conch. Cab. p. 196. pl. 24, f. 17. D.F.

Type in British Museum.

Hab. Natal. Pietermaritzburg (fide M. \& P.).

Founded on a single specimen. 
40. Ennea dunkeri, Pfeiffer.

1855 Ennea dunkeri, Pfr., Mal. Blätt. ii. p. 173. D.

1856

1859

1885
" Novit. Conch. i. p. 73. pl. 20, f. 11-13. D.F.

" Mon. Hel. iv. p. 339. D.

" Tryon, Man. of Conch. i. p. 98. pl. 19,

1898 Ennea differens, Stur., S.A. Moll. p. 20. pl. 1, f. 5, 6. D.F.

1904 , Kob., Conch. Cab. p. 202. pl. 25, f. 6,7 . D.F.

„Ennea dunkeri, Pfr., Kob., Conch. Cab. p. 193.pl.24, f. 9, 10. D.F. Type of dunkeri in Stettin Museum; differens in Naturh. Hofmus. Vienna.

Hab. Natal. Port Natal (Plant). Durban (differens, Penther). Tyeloti, near Botha's Hill; Pinetown; Table Mountain; Lower Umkomaas (Burnup).

41. Ennea elliptica, Melv. \& Pons.

[S.A.M.]

1898 Ennea elliptica, M. \& P., A.M.N.H. ii. p. 126. pl. 7, f. 2. D.F. 1904 Kob., Conch. Cab. p.180.pl.23,f.4. D.F.

Type in British Museum.

Hab. NATAL. Pietermaritzburg; Nottingham Road; Dargle; Karkloof; Hilton Road; Tyeloti, near Botha's Hill; Zwaartkop; Richmond; Edendale (Burnup).

42. Ennea eshowensis, Melv. \& Pons.

1909 Ennea eshowensis, M. \& P., A.M.N.H.iv. p. 487. pl. 8, f. 4. D.F. Type in British Museum.

Hab. Zululand. Eshowe (Lady Saunders).

43. Ennea euthyma, Melv. \& Pons.

1893 Ennea enthymia, M. \& P., A.M.N.H. xii. p. 107. pl. 3, f.10. D.F. $1898 \quad$ ", $\quad$ Stur., S.A. Moll. p. 21. N.

$1901 \quad$ " " $\quad$ " Kob.,Conch.Cab.p.197.pl.24,f.19.D.F.

Type in British Museum.

Hab. Natal. Thornybush, near Pietermaritzburg (Burnup).

A larger variety is found at Lower Umkomaas (Burnup). 
44. Ennea eximin, Melv. \& Pons.

1898 Ennca eximia, M. \& P., A.M.N.H. i. p. 28. pl. 8, f. 8. D.F. $1904 \quad$ " " Kob., Conch.Cab.p.184.pl.23,f.13. D.F. $1909 \quad$ " $\quad$ A.M.N.H.iv. p. 487. pl. 8, f. 6. N.F.

Type in British Museum.

Hab. Transvaal. Between Barberton and Delagoa Bay (fide M. and P.).

45. Ennea farquhari, Melv. \& Pons.

1895 Ennea farquhari, M. \& P., A.M.N.H. xvi. p. 478. pl. 18, f. 3-5. D.F.

1904

Kob., Conch. Cab. p. 186. pl. 23, f. 17. D.F.

Type in British Museum.

Hab. Cape of Good Hope. Grabamstown (Farquhar).

E. microthanma, M. \& P., is very nearly allied to this species.

46. Ennea foriclusa, Melv. \& Pons.

1901 Ennea forichusa, M. \& P., A.M.N.H. viii. p. 316. pl. 2, f. 3. D.F. 1904 Kob., Conch. Cab.p.236.pl.32,f.23. D.F.

Type in British Museum.

Hab. Natal. Table Mountain, about 12 miles from Pietermaritzburg (Burnup).

47. Ennea formosa, Melv. \& Pons.

1898 Ennea formosa, M. \& P., A.M.N.H. ii. p. 126. pl. 7, f. 3. D.F. 1904 Kob., Conch. Cab. p. 183. pl. 23, f. 12 . D.F.

Type in British Museum.

Hab. Natal. Durban (Quekett). Pietermaritzburg (Burnup; Connolly). Karkloof (Taynton).

Some doubt exists as to whether the locality originally givenDurban -is correct.

48. Ennea genialis, Melv. \& Pons.

[S.A.M. ]

1903 Ennea genialis, M. \& P., A.M.N.H. xii. p. 598. pl. 31, f. 14. D.F. 1907 " vallaris, " " $\quad$ xix.p. 96. pl.6, f. 5. D.F.

Types in British Museum.

Hab. Zululand. Patana; Lower Umfolosi Drift (Burnup). Melmoth (vallaris, Miss Hickey). 
Though the type of vallaris is a little smaller and more coarsely ribbed than that of genialis, the two species appear to be inseparable.

49. Ennea Gouldi, Pfeiffer.

[S.A.M.]

1855 Ennea gouldi, Pfr., Mal. Blätt. ii. p. 174. D.

1856

1859

" Novit. Conch. i. p. 72. pl. 20, f. 7-10. D.F.

1885

" Mon. Hel. iv. p. 339. D.

,

"Tryon, Man. of Conch. i. p. 98. pl. 19, f. $13 . D . F$.

1904

Type in Stettin Museum.

Kob., Conch. Cab.p. 193.pl. 24,f.11,12. D.F.

Hab. Natal. Durban (Plant). Isipingo (Penther). Lower Umkomaas; Equeefa; Alexandra Junction; Umbogintwini; Tongaat; Pinetown; Ntimbankulu (Burnup).

var. Excedens, Sturany.

1898 Ennea gouldi, Pfr., var. excedens, Stur., S.A. Moll. p. 18. pl. 1, f. 3. D.F.

Type in Naturh. Hofmus. Vienna.

Hab. Natal. Durban (Penther). Lower Umkomaas (Burnup).

50. Ennea hickeyana, Melv. \& Pons.

1901 Ennea hickeyana, M.\& P., A.M.N.H. viii.p. 317. pl. 2, f. 4. D.F. $1904 \quad$ " $\quad$ Kob., Conch. Cab.p.243.pl.33,f.5. D.F.

Type in British Museum.

Hab. Natal. Biggarsberg (Miss Hickey).

51. Ennea himerothales, Melv. \& Pons.

1903 Ennea himerothales, M. \& P., A.M.N.H. xii. p. 599. pl. 31, f. 13 . D.F.

Type in British Museum.

Hab. Natal. Port Shepstone (Burnup). Durban (in coll. Ponsonby).

52. Ennea hypsona, Melv. \& Pons.

1909 Ennea hypsoma, M. \& P., A.M.N.H. iv. p. 488. pl. 8, f. 7. D.F Type in British Museum.

Hab. Cape of Good Hope. Traps Valley, Bathurst (Farquhar). 
53. Ennea infans, Craven.

1880 Ennea infans, Crvn., P.Z.S. p. 616. pl. 57, f. 6. D.F.

1885

"Tryon, Man. of Conch. i. p. 102.pl. 20, f. $55,56, D . F$.

1893 Ennea collicri, M. \& P., A.M.N.H. xi. p. 23. pl. 3, f. 13 . D.F. 1904 Kob., Conch. Cab. p. 188.pl.23,f.21. D.F. "Ennea infans, Crvn., Kob., Conch. Cab. p. 136. pl. 19, f. 18. D.F. Types in British Museum.

Hab. Transvaal. Lydenburg (Craven). Pretoria District (infans and collieri) (plentiful). Johannesburg (Gould).

Although the type of collieri is longer than that of infans, the two species merge into one another; E. wottoni, M. \& P., is also nearly allied to Craven's shell.

54. Ennea infrendens, von Martens.

[S.A.M.]

1866 Pupa (Ennea) infrendens, von Mts., Mal. Blätt. xiii. p. 110. pl. 3, f. 10-12. D.F.

1868 Ennea infrendens, von Mts., Pfr. Mon. Hel. v. p. 454. D.

1885 ", "Tryon, Man. of Conch. i. p. 98 pl. 19, f. 86. D.F.

$1898 \quad$ " $\quad$ Stur., S.A. Moll. p. 23. N.

$1904 \quad$ " $\quad$ Kob., Conch. Cab. p. 215. pl. 27, f. 1. D.F.

Type-ubi?

Hab. Natal (Queinzius). Durban (Penther). Greenwood Park (Miss Hickey). Equeefa; Tongaat Beach (Burnup).

Ennea claustraria, M. \& P., is very closely allied to this species if not merely a variety of it.

55. Ennea ingens, Sturany.

1898 Ennea ingens, Stur., S.A. Moll. p. 23. pl. 1, f. 9. D.F.

$1904 \quad$ " $\quad$ Kob., Conch. Cab. p. 137.pl. 19, f. 23. D.F. Type in Naturh. Hofmus. Vienna.

Hab. Natal. Durban (Penther).

56. Ennea instabilis, Sturany.

[S.A.M.]

1898 Ennea instabilis, Stux., S.A. Moll. p. 24. pl. 1, f. 13. D.F.

$1904 \quad$ " Kob., Conch. Cab.p. 184. pl.23,f.14. D.F. 
Type in Naturh. Hofmus. Vienna.

Hab. Natal. Isipingo; Durban (Penther). Equeefa; Lower Umkomaas; Coast Bush generally, from Port Shepstone to Tongaat; Pinetown; Table Mountain (Burnup).

57. Ennea isipingöensis, Sturany.

1898 Ennea isipingöensis, Stur., S.A. Moll. p. 27. pl. 1, f. 19. D.F. 1904 " Kob., Conch. Cab. p. 201. pl. 25, f. 2-5. D.F.

Type in Naturh. Hofmus. Vienna.

Hab. Natal. Isipingo (Penther). Dargle; Equeefa; Karkloof; Inhluzani ; Howick; Mid-Illovo ; Ntimbankulu ; Pietermaritzburg ; Edendale; Table Mountain; Tyeloti, near Botha's Hill (Burnup).

varr. Discrepans, similinim, and CYLINDrica, Sturany.

1898 Ennea isipingöensis, var. discrepans, simillima, and cylindrica, Stur., S.A. Moll. p. 27. pl. 1, ff. 20, 21, and 22 respectively. D.F. Types in Naturh. Hofmus. Vienna.

Hab. Natal. Isipingo (Penther).

58. Ennea johannesburgensis, Melv. \& Pons, [S.A.M.] 1907 Ennea johannesburgensis, M. \& P., A.M.N.H. xix. p. 95. pl. 6, f. 2. D.F.

Type in British Museum.

Hab. Transvaal. Johannesburg (Johnson; McBean). Potchefstroom (Miss Cachet). Standerton (Connolly).

Orange Free State. Bloemfontein (Connolly).

59. Ennea Juxtidens, Melv. \& Pons.

1899 Ennea juxtidens, M. \& P., A.M.N.H. iv. p. 195. pl. 3, f. 2. D.F. $1904 \quad " \quad$ Kob., Conch. Cab, p. 234. pl. 32,

Type in British Museum. f. $14 . D . F$.

Hab. Orange Free State. Van Reenen (Mrs. Quekett).

60. Ennea kosiensis, Melv. \& Pons.

1908 Ennea kosiensis, M. \& P., A.M.N.H. i. p. 130. pl. 7, f. 2. D.F. Type in British Museum.

Hab. Zululand. Kosi Bay (Burnup). 
1855 Ennea kraussi, Pfr., Mal. Blätt. ii. p. 174. D.

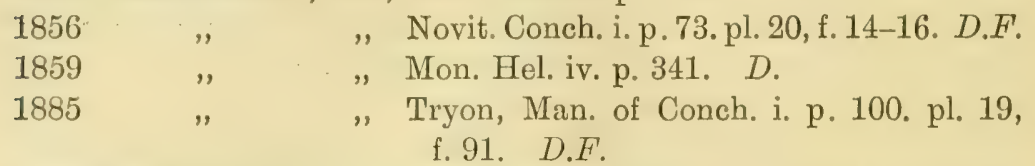

1898 Ennea sejuncta, Stur., S.A. Moll. p. 24. pl. 1. f. 10-12. D.F. 1904 " " Kob.,Conch.Cab.p.204.pl.25,f.15-17.D.F. "Ennea kraussi, Pfr., Kob., Conch. Cab. p. 134. pl. 19, f. 19, 20. D.F.

Type of kraussi in Stettin Museum; sejuncta in Naturh. Hofmus. Vienna.

Hab. Natal. Port Natal (Plant). Durban (sejuncta, Penther). Lower Umkomaas; Tongaat Beach; Umbogintwini; Pinetown (Burnup).

62. Ennea Labyrinthea, Melv. \& Pons.

1895 Ennea labyrinthea, M. \& P., A.M.N.H. xvi. p. 479. pl. 18, f. $7,8$. D.F.

1898 " labyrinthica, M. \& P., Stur., S.A. Moll.p. 29 (Err.typ.). 1904 ", labyrinthea, M. \& P., Kob., Conch. Cab. p. 224. pl. 28, f. 2, 3. D.F.

Type in British Museum.

Hab. CaPe of Good Hope. Grahamstown (Farquhar).

\section{Ennea leppani, Sturany.}

1898 Ennea leppani, Stur., S.A. Moll. p. 28. pl. 2, f. 23-25. D.F. $1904 \quad, \quad, \quad$ Kob., Conch.Cab.p.137.pl.19,f.21,22. D.F.

Type in Naturh. Hofmus. Vienna.

Hab. CAPE of Good Hope. Albany District (Penther).

64. Ennea magnolia, sp. nov., pl. 2, f. 1. [S.A.M.]

Shell small, elongate, cylindriform, subrimate, smooth, glossy, vitreous, transparent. Spire narrow, produced, sides parallel, apex rounded. Whorls 6 , flattish, with practically no sculpture; the apical small, remainder almost equal in circumference and increasing very slowly in distance between sutures. Suture shallow, margined below. Aperture subquadrate; peristome white, slightly thickened and reflexed, with 2 weak teeth; the parietal, short and pointed; the other, little more than a protuberance caused by a. slight 
straightening, half-way down, of the outer lip, which forms an obtuse forward angle at this point.

Shell $4.75 \times 1.4$; aperture $95 \times \cdot 85$; last whorl $1.8 \mathrm{~mm}$.

Type in British Museum.

Hab. Rhodesia. Victoria Falls (Connolly; Warren).

The contour of the spire, regular in the type, is frequently irregular, being sometimes crooked, and sometimes. swollen towards the apex. The present species resembles E. cimolia, M. \& P., in form and substance, but is more than twice as large, with much weaker dentition.

65. Ennea margarette, Melv. \& Pons.

1895 Ennea margaretta, M. \& P., A.M.N.H. xvi. p. 479. pl. 18, f. 1. D.F.

1904 Kob., Conch. Cab. p. 185. pl. 23, f. 16. D.F.

Hab. Cape of Good Hope. Grahamstown (Farquhar).

Founded on a single specimen, not fully mature, whose present whereabouts has not been ascertained.

66. Ennea mariæ, Melv. \& Pons,

1892 Enrea maria, M. \& P., A.M.N.H. ix. p. 92. pl. 6, f. 12. D.F. $1904 \quad$, $\quad$ "Kob., Conch. Cab.p.220.pl.27,f.11. D.F.

Type in British Museum.

Hab. Cape of Good Hope. Craigie Burn, Somerset East (Miss Bowker).

67. Ennea maritzburgensis, Melv. \& Pons. [S.A.M.] 1893 Ennea maritzburgensis, M. \& P., A.M.N.H. xii. p. 107.pl. 3, 1904 , f. 11. D.F. " Kob., Conch. Cab. p. 198. pl. 24, f. 21 . D.F.

Type in British Museum.

Hab. Natal. Pietermaritzburg (Quekett; Burnup; Ponsonby).

\section{Ennea menkeana (Pfeiffer).}

1853 Pupa mentreana, Pfr., Mon. Hel. iii. p. 551. D.

1859 Ennea menkeance "N Novit. Conch. i.p.113.pl.32, f.3-5. D.F. 1878 Pupa menkeana ", Sorv., Conch. Icon. pl. 19, f. 176. D.F. (Err. Loc.). 
1885 Ennea menkeana, Pfr., Tryon, Man. of Conch. i. p. 97. pl. 18, f. 79. D.F.

$1904 \quad$ " $\quad$ Kob., Conch.Cab.p.191.pl.24,f.6. D.F'.

Hab. Natal. Port Natal (in coll. Menke).

69. Ennea microthauma, Melv. \& Pons.

1899 Ennea microthauma, M. \& P., A.M.N.H. iv.p. 194. pl. 3, f. 1. D.F. 1904 " Kob., Conch. Cab. p. 234. pl. 32, f. 13. D.F.

Type in British Museum.

Hab. Cape of Good Hope. Grahamstown (Langley).

Extremely closely allied to the variable $E$. farquhari, M. \& P.

70. Ennea minuta, Morelet.

1889 Ennea pusilla, Morel., J. de C. xxxvii. p. 13. pl. 2, f. 3. D.F. " " minuta (=pusilla, Morel., 1889, nec 1881), Morel., J. de C. xxxvii. p. 200.
1898 " ambigua, Stur., S.A. Moll. p. 29. N.

1904 " minuta, Morel., Kob., Conch. Cab. p. 212. pl. 26, f. 2224. D.F.

Type in British Museum.

Hab. Cape of Good Hope. Port Elizabeth (Crawford). Tharfield (Miss Bowker).

71. Ennea montana, Melv. \& Pons.

1903 Ennea montana, M. \& P., A.M.N.H. xii. p. 599. pl. 31, f. 15. D.F.

Type in British Museum.

Hab. CAPE of Good Hope. Mountain Drive, Grahamstown (Farquhar).

72. Ennea multidentata, Sturany.

1898 Ennea multidentata, Stur., S.A. Moll. p. 25. pl. 1, f. 16. D.F'. $1904 \quad$ ", Kob., Conch. Cab. p. 195. pl. 24, f. 15 . D.F.

Type in Naturh. Hofmus. Vienna.

Hab. Natal. Isipingo (Penther). Equeefa; Durban; Alexandra Junction (Burnup).

73. Ennea uunita, Melv. \& Pons.

1892 Ennea munita, M. \& P., A.M.N.H. ix. p. 86. pl. 6, f. 5. D.F. $1904 \quad$ " $\quad$ Kob., Conch. Cab.p.217.pl.27, f.6. D.F. 
Type in British Museum.

Hab. CAPE of Good Hope. Griqualand East (Sykes).

74. Ennea natalensis, Craven.

[S.A.M.]

1880 Ennea natalensis, Crvn., P.Z.S. p. 619. pl. 57, f. 7. D.F.

1885

1904
"Tryon, Man. of Conch. i. p. 100. pl. 20, f. 57, 58. D.F.

" Kob., Conch. Cab. p. 232. pl. 28, f. 17, 18. D.F.

Type in British Museum.

Hab. Natal. Durban Harbour (Craven; Penther). Isipingo (Penther). Scottburgh; Lower Umkomaas; Port Shepstone; Equeefa; Alexandra Junction; Umbogintwini; Ntimbankulu; Tongaat Beach (Burnup).

Zululand. Dukuduku Forest (Toppin).

75. Ennea nonotiensis, Melv. \& Pons.

1894 Ennea nonotiensis, M. \& P., A.M.N.H. xiv. p. 95. pl. 1, f. 15. D.F. 1904 " Kob., Conch. Cab. p. 181. pl. 23, f. 6 . D.F.

Type in British Museum.

Hab. Natal. Nonoti (Burnup).

76. Ennea obovata, Pfeiffer.

1855 Ennea obovata, Pfr., P.Z.S. p. 9. D.

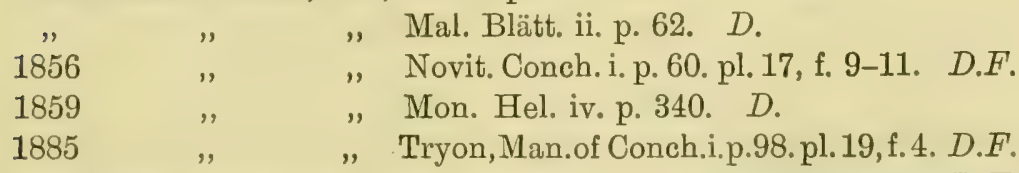
1898 Ennea ampullacea, Stur., S.A. Moll. p. 24. pl. 1, f. 14, 15. D.F. $1904 \quad$ " $\quad$ Kob., Conch. Cab. p. 204. pl. 25, f. 18, 19. D.F.

"Ennea obovata, Pfr., Kob., Conch. Cab.'p. 194.pl. 24, f. 13. D.F. Type of obovata in British Museum; ampullacea in Naturh. Hofmus. Vienna.

Hab. Natal. Isipingo; Durban (Penther). Equeefa; Lower Umkomaas; Pinetown; Ntimbankulu ; Inchanga; Table Mountain ; Karkloof (Burnup).

Originally described as from Liberia, but apparently in error, as no authentic record of its appearance there is in existence. 
77. Ennea oppugnans, Melv. \& Pons.

1909 Emnea oppugnans, M. \& P., A.M.N.H.iv. p. 488. pl. 8, f. 8. D.F. Type in British Museum.

Hab. CAPE OF Good Hore. Traps, Valley, Bathurst (Farquhar).

78. Ennea parallela, Melv. \& Pons.

1909 Ennea parallela, M. \& P., A.M.N.H. iv. p. 489. pl. 8, f. 9. D.F. Type in British Museum.

Hab. CAPE of Good Hope. Grahamstown (Farquhar).

79. Ennea Pentheri, Sturany.

1898 Ennea pentheri, Stur., S.A. Moll. p. 30. pl. 2, f. 32, 33. D.F. 1904 " " Kob., Conch. Cab. p. 242. pl. 28, f. 22, 23. D.F.

Type in Naturh. Hofmus. Vienna.

Hab. NataL. Isipingo (Penther).

Cape of Good Hope. Traps Valley, Bathurst (Farquhar).

Closely allied to, but much smaller than, E. cimolia, M. \& P.

80. Ennea pentodon, Morelet.

[S.A.M:]

1889 Ennea natalensis, Morel., J. de C., xxxvii. p. 11. pl. 2, f. 1. D.F.

", " pentodon (=natalensis, Morel., 1889, nec Crvn., 1880), Morel., J. de C. xxxvii. p. 200.

1898 " binominis, Stur., S.A. Moll. p. 18. N.

1904 " pentodon, Morel., Kob., Conch. Cab. p. 211. pl. 26, f. 1921. D.F.

Type in British Museum.

Hab. Cape of Good Hope. Port Elizabeth (Crawford).

81. Ennea periploca, Melv. \& Pons.

1909 Ennea periploca, M. \& P., A.M.N.H. iv. p. 489. pl. 8, f. 10. D.F. Type in British Museum.

Hab. Cape of Good Hope. Boschberg Mountain, Somerset East (Farquhar).

82. Ennea Perissodonta, Sturany.

1898 Ennea perissodonta, Stur., S. A. Moll. p. 26. pl. 1, f. 18. D.F. $1904 \quad$ „ Kob., Conch. Cab.p. 201. pl. 25, f. 1. D.F.

Type in Naturh. Hofmus. Vienna.

Hab. Lorenzo Marques. Delagoa Bay (Penther).

Founded on a single example. 
83. Ennea Perspicua, Melv. \& Pons.

1893 Entrea perspicua, M. \& P., A.M.N.H. xi. p. 23. pl. 3, f. 12. D.F. 1904 Kob., Conch. Cab. p. 188. pl. 23, f. 22. D.F.

Type in British Museum.

Hab. Transvaal. Middelburg (Crawford).

84. Ennea Perspicueformis, Sturany.

1898 Ennea perspicuaformis, Stur., S.A. Moll.p. 17.pl. 1, f. 2. D.F. 1904 " Kob., Conch. Cab. p. 189. pl. 23, f. 23. D.F.

Type in Naturh. Hofmus. Vienna.

Hab. Lorenzo Marques. Delagoa Bay (Penther)

Described from a single specimen, only differing from E. perspicua, M. \& P., in its smaller size and fewer whorls.

\section{EnNea Pfeifferi (Krauss).}

1841 Pupapfeifferi, Krs., Küst., Conch. Cab.p.87.pl.12, f. 17-19. D.F. 1848 " Südafr. Moll. p. 79. $D$.

" $\quad$ " $\quad$ " Pfr., Mon. Hel. ii. p. 352. D.

1885 Ennea pfeifferi, Krs., Tryon, Man. of Conch. i. p. 101. pl. 19, f. 98 . D.F.

$1904 \quad$ " $\quad$ Kob., Conch. Cab.p.133.pl.19, f.12. D.F.

Type in Stuttgart Museum.

Hab. Cape of Good Hope. Potteberg; Swellendam (Krauss).

var. Mrniata, Krauss.

1848 Pupa pfeifferi, var. miniata, Krs., Südafr. Moll. p. 79. D.

" " " " , Pfr., Mon. Hel. ii. p. 353. D. Hab. ? Transvaal. Mount Mohapaani (Wahlberg).

86. Ennea phragma, Melv. \& Pons.

1907 Ennea phragma, M. \& P., A.M.N.H. xix. p. 95. pl. 6, f. 3. D.F. Type in British Museum.

Hab. Cape of Good Hope. Waku, Cathcart District, near the Klipplatz River (Miss Hickey).

87. Ennea planti, Pfeiffer.

1855 Ennea plonti, Pfr., Mal. Blätt. ii. p. 173. D. 1856

"Novit. Conch. i. p. 72. pl. 20, f. 5, 6. D.F. 
1859 Ennea planti, Pfr., Mon. Hel. iv. p. 337. D.

1878 Pupa planti " Sow., Conch. Icon. pl. 18, f. 169. D.F.

1885 Ennea planti ," Tryon, Man. of Conch.i.p.90.pl.17,f. 25. D.F. $1898 \quad$ " $\quad$ Stur., S.A. Moll. p. 16. N.

$1904 \quad$ " Kob., Conch. Cab.p.178.pl.22, f. 23, 24. D.F. Type in Stettin Museum.

Hab. Natal (Plant). Durban (Penther). Tongaat Beach (Burnup).

88. Ennea Polita, Melv. \& Pons.

1893 Ennea polita, M, \& P., A.M.N.H. xii. p. 108. pl. 3, f. 12. D.F. $1904 \quad$ " $\quad$ Kob., Conch. Cab. p. 195. pl.24,f.14. D.F.

Type in British Museum.

Hab. Cape of Good Hope. Tharfield (Miss Bowker).

89. Ennea premnodes, Sturany.

1901 Ennea premnodes, Stur., Ann. Hofmus. Wien, xvi.p. 69, f. 5. D.F. 1905 " Kob., Conch. Cab. p. 344. pl. 32 (1904), f. 19. D.F.

Type in Naturh. Hofmus. Vienna.

Hab. Cape of Good Hope. Albany District (Penther).

90. Ennea pulchella, Melv. \& Pons.

1893 Ennea pulchella, M. \& P., A.M.N.H. xii. p. 108. pl. 3, f. 13. D.F. 1904 ", Kob., Conch. Cab. p. 197. pl. 24,

. Type in British Museum. f. $18 . D . F$.

Hab. Natal. Pietermaritzburg (Burnup).

91. Ennea Queketti, Melv. \& Pons.

[S.A.M.]

1896 Ennea queketti, M. \& P., A.M.N.H. xviii. p. 315. pl. 16, f, 2. D.F. $1898 \quad$ " $\quad$ Stur., S.A. Moll. p. 16. N.

1904 Ennea quecketti ," Kob., Conch. Cab. p. 241. pl. 29,

Type in British Museum.

f. 20. D.F.

Hab. Natal. Umzinto (Canon Pennington). Isipingo(Penther). Equeefa; Lower Umkomaas; Durban (Burnup). 
92. Ennea regularis, Melv. \& Pons.

[S.A.M.]

1893 Ennea regularis, M. \& P., A.M.N.H. xi. p. 22. pl. 3, f. 11. D.F. 1904

Kob., Conch. Cab. p. 187. pl. 23, f. 20. D.F.

Type in British Museum.

Hab. Natal. Pietermaritzburg; Albert Falls, Umgeni River (Burnup). Howick (Cregoe).

93. Ennea Rhodesiana, sp. nov., pl. 2, f. 2. [S.A.M.]

Shell small, shortly cylindrical, rimate, rather glossy, translucent, milky olivaceous. Spire short, apex rounded. Whorls 5, convex, very gradually increasing, covered, after the first two, with welldefined, subdistant striæ. Suture crenulate. Aperture subquadrate, furnished with four dental processes; one tooth, thick and blunted, deeply inset at the upper angle of the columellar region; one, small and pointed, slightly to the left centre of the base; another, of greater length, pointing inwards and downwards, halfway up the outer lip; and a prominent, somewhat protruding plait, slightly concave on its right side, at the junction of the outer lip and paries. Peristome white, thickened and reflexed.

Shell $3.7 \times 1.8$; aperture $90 \times 1 \cdot 0$; last whorl $2 \mathrm{~mm}$.

Type in South African Museum.,

Hab. RHodesra. Victoria Falls (Connolly).

Easily distinguishable from $E$. johannesburgensis, M. \& P., whose dentition is very similar.

\section{Ennea Rogersi, Melv. \& Pons.}

1898 Ennea rogersi, M. \& P., A.M.N.H. i. p. 26. pl. 8, f. 6. D.F. $1904 \quad$ " $\quad$ Kob., Conch. Cab.p. 179.pl.23,f.1. D.F.

Type in British Museum.

Hab. CaPe of Good Hope. Cradock Commonage (Farquhar, in coll. Rogers). Teafontein, Grahamstown (in coll. Fulton).

95. Ennea scrobiculata, Melv. \& Pons.

1892 Ennea scrobiculata, M. \& P., A.M.N.H. ix. p. 93.pl.6, f. 8. D.F. 1904 " Kob., Conch. Cab. p. 218. pl. 27, f. $8 . D . F$.

Type in British Museum.

Hab. Natal (Layard). 
1898 Ennea separata, Stur., S.A. Moll. p. 20. pl. 1, f. 7, 8. D.F.

1904 " Kob., Conch. Cab.p. 202.pl.25,f.8,9. D.F.

Type in Naturh. Hofmus. Vienna.

Hab. Natal. Isipingo; Durban Bluff (Penther). Equeefa; Pietermaritzburg; Table Mountain; Lower Umkomaas; Umbogintwini ; Port Shepstone; Ntimbankulu (Burnup).

97. Ennea socratica, Melv. \& Pons.

1893 Ennea socratica, M. \& P., A.M.N.H. xii. p. 109. pl. 3, f.-14. D.F. $1904 \quad$ " . Kob.,Conch.Cab.p.198.pl.24,f.20. D.F.

Type in British Museum.

Hab. NataL. Pietermaritzburg (fide M. \& P.).

Founded on a single specimen.

98. Ennea syuvia, Melv. \& Pons.

1903 Ennea sylvia; M. \& P., A.M.N.H. xii. p. 599. pl. 31, f. 4. D.F.

Type in British Museum.

Hab. CAPE of Good Hope. Maeström Forest, Bedford (Farquhar).

Natal. Examples from Natal, which have been attributed to this species, are now proved to be distinct.

99. Ennea tharfieldensis, Melv. \& Pons.

1893 Ennea tharfieldensis, M. \& P., A.M.N.H.xii.p. 109.pl.3, f.15. D.F. 1904 " Kob., Conch. Cab. p. 200. pl. 24, f. 24 . D.F.

Type in British Museum.

Hab. Cape of Good Hope. Tharfield (in coll. Layard). Kowie (Falquhar).

100. Ennea themodonta, Melv. \& Pons.

1892 Ennea thelodonta, M. \& P., A.M.N.H. ix. p. 85. pl. 6, f. 4. D.F. $1904 \quad$ " Kob., Conch. Cab. p. 216. pl. 27,

Type in British Museum.

$$
\text { f. 3. D.F. }
$$

Hab. Cape of Good Hope. Port Elizabeth (Langley; Crawford). 
101. Ennea transiens, Sturany.

1898 Ennea transiens, Stur., S.A. Moll. p. 19. pl. 1, f. 4. D.F. 1904

Type in Naturh. Hofmus. Vienna. ", Kob., Conch. Cab.p. 228. pl. 28,f,10. D.F.

Hab. Natal. Durban and neighbourhood (Penther).

Very nearly allied to, if not identical with, wahlbergi, Krs.

102. Ennea triglochis, Melv. \& Pons.

1903 Ennea triglochis, M. \& P., A.M.N.H. xii. p. 600. pl. 31, f. 11. D.F. " $"$ virgo $" \quad " \quad$ ", f. 10. D.F. Types in British Museum.

Hab. Natal. Botha's Hill (Burnup).

Zululand, Lower Umfolosi Drift (Burnup). Melmoth (Miss Hickey). Dukuduku Forest and Kosi Bay (Toppin).

Although the types differ slightly, intermediate forms are found of these two shells, which are now admitted to be conspecific.

103. Ennea vandenbroecki, Melv. \& Pons.

1893 Ennea vandenbroeckii, M. \& P., A.M.N.H. xii. p. 110. pl. 3, f. $16 . \quad D . F$.

1904

Kob., Conch. Cab. p. 199. pl. 24, f. 22. D.F.

Type in British Museum.

Hab. Natal (ex, coll. Vandenbroeck).

Founded on two specimens.

104. Ennea vanstaadensis, Melv. \& Pons.

1893 Ennea vanstaadensis, M. \& P., A.M.N.H.xii.p.110.pl.3,f.17. D.F. 1904 . " Kob., Conch. Cab. p. 199. pl. 24,

Type in British Museum. f. 23 . D.F.

Hab. Cape of Good Hope. Van Staadens River, Port Elizabeth (Crawford).

105. Ennea vitreola, Melv. \& Pons.

1908 Ennea vitreola, M. \& P., A.M.N.H. i. p. 130. pl. 7, f. 3. D.F. Type in Natal Museum, Pietermaritzburg.

Hab. Natal. Hilton Road (Burnup). 


\section{ENNeA WAHLBERgi (Krauss).}

1848 Pupa wahlbergi, Krs., Südafr. Moll. p. 80. pl. 5, f. 5. D.F.

," ", wahlbergii ," Pfr., Mon. Hel. ii. p. 352. D.

1855 " wahlbergi " Küst., Conch. Cab. p.158.pl.19,f.6-9. D.F.

1878 " walbergi, Pfr., Sow., Conch. Icon. pl. 20, f. 187. D.F.

1885 Ennea wahlbergi, Krs., Tryon, Man. of Conch. i. p. 96. pl. 19, f. 99 . D.F.

$1904 \quad$ ", Kob.,Conch.Cab.p.190.pl.24,f.3,4. D.F.

Type in Stuttgart Museum.

Hab. NataL (Wahlberg). Durban and neighbourhood; Isipingo (Penther, fide Sturany).

Founded on two specimens, which differed from each other both in sculpture and dentition.

107. Ennea warrent, Melv. \& Pons.

[S.A.M.]

1903 Ennea warrenii, M. \& P., A.M.N.H. xii.p. 601. pl. 31, f. 7. D.F. Type in British Museum.

Hab. Zululand. Lower Umfolosi Drift (Warren). Makowe (Crosly). Melmoth (Miss Hickey).

Cape of Good Hope. Port St. John's (Shortridge).

108. Ennea wottoni, Melv. \& Pons.

1895 Ennea wottoni, M. \& P., A.M.N.H. xvi.p. 479. pl. 18, f. 6. D.F. $1898 \quad$ " " Stur., S.A. Moll. p. 17. N.

$1904 \quad$ " $\quad$ Kob., Conch.Cab.p.186.pl.23,f.18. D.F.

Type in British Museum.

Hab. Cape of Good Hope. Grahamstown (Farquhar). Albany District (Penther).

Very closely allied to, if not inseparable from, infans, Crvn.

109. Ennea xysila, Melv. \& Pons.

[S.A.M.]

1907 Ennea xysila, M. \& P., A.M.N.H. xix. p. 97. pl. 6, f. 6. D.F. "Types in British Museum. var. hyalina, M.\&P.,ibid.pl.6, f.6a. N.F.

Hab. Transvaal. Johannesburg (Johnson; McBean). Pretoria and surrounding district (McBean; Connolly). Heidelberg (Miss Livingston). 
Although the extremes of form vary greatly, almost every intermediate grade can be traced, so that the varietal name is hardly retainable.

110. Ennea zelota, Melv. \& Pons.

[S.A.M.]

1907. Ennea zelota, M. \& P., A.M.N.H. xix. p. 97. pl. 6, f. 7. D.F.

Type in British Museum.

Hab. Natal. Port Shepstone; Equeefa (Burnup).

\section{Genus STREPTOSTELE, Dohrn, 1866.}

(Mal. Blätt. xiii. p. 128.)

Type of Genus, S. lotophaga (Morel).

11. Streptostele heria, sp. nov., pl. 2, f. 3. [S.A.M.]
[S.M.

Shell very small, elongate, narrowly rimate, slightly glossy, translucent, milky olivaceous. Spire produced, tapering, very narrow, apex rounded. Whorls 7, gradually increasing, little convex, but impressed at the suture; the first 2 smooth, remainder beautifully sculptured with rather coarse, irregular, almost straight striæ. Suture deep. Aperture short, piriform, virtually edentulate, the only processes being an almost imperceptible parietal callosity, and an incurvation of the outer lip about one-third of its length below the suture. Peristome not thickened, thinly reflexed.

Shell $4.5 \times 1.3$; aperture $9 \times \cdot 8$; last whorl $1.5 \mathrm{~mm}$.

Type in British Museum.

Hab. RHodesia. Victoria Falls (Connolly).

This beautiful little species belongs to a group of Central African shells which have been placed of late years in Streptostele. As their columella is not achatinoid, as it should be in Dohrn's genus, it is probable that a new section will have to be founded for them.

FAмirx RHYTIDID A , Pilsbry, 1893.

(Man. of Conch. viii. p. 135.)

Genus NATALiNA, Pilsbry, 1893.

(Man. of Conch, viii. p. 135.)

(=Aerope, Albers, 1860, non Leach, 1813.)

Type of Genus, N. caffra (Fér.).

The species which follow have been variously attributed to Natalina, or to Rhytida, Albers (Die Helic., 1860, p. 89. Type greenwoodi, Gray), and its subgenera, Macrocycloides, von Martens 
(Preuss. Exp. nach. Ost.-Asien., 1867, Zool. ii. p. 259. Type obscurata, Ad. \& Rve.), and Afrorhytida, Möllendorff (Conch. Cab., 1903, p. 61. Type knysnacnsis, Pfr.).

Very little is known of most of the animals, and subdivision of the shells, on conchological grounds alone, is by no means satisfactory. Future research will doubtless provide anatomical grounds for reclassification, and establish the suggested subgenera on a permanent basis. Meanwhile, to facilitate reference, it is more convenient to place the species in alphabetical sequence under Natalina.

\section{Natalina arguta, Melv. \& Pons.}

1907 Natalina arguta, M. \& P., A.M.N.H. xix. p. 98. pl. 6, f. 8. D.F. Type in British Museum.

Hab. Cape of Good Hope. East London (Burnup).

\section{Natalina beyrichi (von Martens).}

1890 Aerope beyrichi, von Mts., Sitz.-Ber. Ges. Nat. Fr. Berlin, p. 85. $D$.

Conch. Mitth. iii, 3. p. 1. D.

1894

1897.

\section{,}

,

1903 Natalina, beyrichi

Type in Zool. Mus. Berlin.

Hab. Cape of Good Hope. Pondoland (Beyrich).

\section{Natalina caffra (Férussac).}

1821 Helix cafra, Fér., Tabl., Syst. Moll. pt. 3. p. 29 (or 25.) N. 1838 „ Desh., Hist. Nat. An. s. Vert. viii. p. 107. D. 1848 Helix caffra ", Krs., Südafr. Moll. p. 75. N. " $\quad$ " " Pfr., Mon. Hel. i. p. 40. D. 1850 Helix cafra ," Desh., Hist. Nat. Moll. i. p. 198. pl. 9A, f. 8. D.F.

1851 Helix caffra ," Rve., Conch. Icon. pl. 40, f. 179. D.F. 1851, 52 " ‥ ", Pfr., Conch. Cab. p. 52. pl. 74, f. 1-3. D.F. 1880 Rhytida caffra, Fér., Gibb., Journ, of Conch. iii. p. 95. D. 1885 Aerope " "Tryon, Man. of Conch. i. p. 131, pl. 25, f. 13. D.F. 
1895 Natalina caffra, Fér., M. Woodw., Proc. Mal. Soc. i. p. 270. pl. 17, f. 1-9. $A$.

1903 " " " Mlldff., Conch.Cab.p.20.pl.3,f.4-7. D.F.

Type-ubi?

Hab. Cape of Good Hope. Caffraria (Delalande). Uitenhage (fide Férussac). Algoa Bay (Krauss). Port Elizabeth (Crawford). Grahamstown; Bedford; Bathurst; Kowie (Farquhar). Somerset East (Miss Borvker). Pirie Mountain (Godfrey).

Transvaal, Between Delagoa Bay and Lydenburg (Wilms).

NataL. Woods near Natal Bay (Krauss). Along the coast from Port Shepstone to Tongaat; Pinetown ; Pietermaritzburg ; Karkloof; Curry's Post (Burnup).

Zululand (in. coll, Layard, who wrote: "This species was sent to me from Natal by the late Mr. H. J. McKen, who told me he procured it on a battlefield in Zululand, feeding on the putrefying corpses of the natives slain in one of their tribal fights.")

var. wesseliana, Maltzan.

1876 Helix caffra, Fér., var. wesseliana, Maltz., Kobelt, Jahrb. d. deutsch. Mal. Ges, iii. p. 149. pl. 5, f. 1. D.F.

1877 Helix caffra, Fér., var. wesseliana, Maltz., Pfr., Mon. Hel. viii. p. $558 . \quad D$.

1885 Aerope caffra, Fér., var. wesselliana, Maltz., Tryon, Man. of Conch. i. p. 131. pl. 25, f. 14. D.F.

1903 Natalina caffra, Fér., var. wesseliana, Maltz., Mlldff., Conch. Cab. p. 21. pl. 3, f. 8. D.F.

Hab. South Africa (fide Kobelt).

Zululand (Toppin).

Lorenzo Marques. Rikatla (Junod).

115. Natalina caffrula, Melv. \& Pons.

1898 Natalina caffrula, M. \& P., A.M.N.H. i. p. 24. pl. 8, f. 1. D.F. $1903 \quad$ " " Stur., S.A. Moll. p. 32. N.

$1903 \quad$ " " Mlldff., Conch. Cab. p. 23. pl. 4,

Type in British Museum.

$$
\text { f. } 4 \text {, 5. D.F. }
$$

Hab. CAPE of Good Hope. Knysna (Pureell). North bank of Zwartkops River, Port Elizabeth (Crawford).

Lorenzo Marques. Matolla (Penther, fide Sturany).

Natal. Durban (Penther, fide Sturany). 
116. Natalina chaptini, Melv. \& Pons.

1894 Natalina chaplini, M. \& P., A.M.N.H. xiv. p. 91.pl.1, f. 3. D.F. 1895 xv. p. 165. pl. 12, f. 5. $F$. 1903 Rhytida (Macrocycloides) chaplini, M. \& P., Mlldff., Conch. Cab. p. 58. pl. 10, f. 15-17. D.F.

Type in British Museum.

Hab. Cape of Good Hope. Kamachs, 5 miles west of Uitenhage (Crawford).

117. Natalina cemotera (Melv. \& Pons).

1892 Helix (Macrocyclis) canotera, M. \& P., A.M.N.H. х. p. 238. pl. 13, f. 2. D.F.

1903 Rhytida (Macrocycloides) canotera, M. \& P., Mlldff., Conch. Cab. p. 59. pl. 10, f. 19. D.F.

Type in British Museum.

Hab. "S. AFRIOA" (Trimen).

CAPE OF Good Hope. Tharfield (Schönland). Grahamstown (Farquhar). Somerset East (Miss Bowker; Purcell). Port Alfred (Crawford). Pirie Mountain (Godfrey).

Natal. Pietermaritzburg; Port Shepstone; Howick (Burnup). Majuba (Connolly).

Zululand. Hlabisa (Burnup). Dukuduku (Toppin).

118. Natalina coerneyensis, Melv. \& Pons.

1894 Natalina coemeyensis, M. \& P., A.M.N.H., xiv. p. 91. pl. 1, f. 2. D.F.

1903 Rhytida (Afrorhytida) coerneyensis, M. \& P., Mlldff., Conch. Cab. p. 64. pl. 11, f. 8. D.F.

Type in British Museum.

Hab. CAPE OF Good Hope. Coerney; Sandflats Station (Crawford).

Natal (in coll. Layard, fide M. \& P.).

$$
\text { 119. Natalina cosmia (Pfeiffer). }
$$

1850 Helix munda, Benson, A.M.N.H. vi. p. 253. D.

1851 " cerea, Pfr. (=munda, Bs. 1850, nec Adams 1849), Pfr., Zeitschr. f. Malak. viii. p. $16 . \quad N$.

1852 " cosmia, Pfr. (=cerea, Pfr. 1851, nec Gould. 1850), Pfr., Zeitschr. f. Malak. ix. p. 112 . N.

1853 " , " , Mon. Hel, iii. p. 94. D. 
1853 Helix cosmia, Pfr., Rve., Conch. Icon. pl. 173, f. $1174 . \quad$ D.F.

1854 " , , Conch.Cab.p.389.pl.143(1852), f.6-8. D.F.

1864 " omphation, Bs., A.M.N.H. xiii. p. $494 . \quad D$.

$1868 \quad$ " $\quad$ " $\quad$ Pfr., Mon. Hel. v. p. 153. D.

1887 „, (Pella) cosmia, Pfr., Tryon, Man. of Conch. iii. p. 106. pl. 20, f. 80. D.F.

1898 Natalina cosmia, Pfr. (=omphation, Bs.), M. \& P., Proc. Mal. Soc. iii. p. 170.

1903 Rhytida (Macrocycloides) cosmia, Pfr., Mlldff., Conch. Cab. p. 60 . pl. 10, f. 23-25. D.F.

Type of cosmia-ubi? Specimens of omphalion, ex auct., in University Museum of Zoology, Cambridge.

Hab. Cape Peninsula. Lion's Head, near Camp's Bay (munda, Benson). Top of Table Mountain, overlooking Cape Town (cerea, Layard). Kalk Bay (Lightfoot). Simonstown (omphalion, "fide Hawkins," Benson).

120. Natalina dumeticola (Benson).

1851 Helix dumeticola, Bs., A.M.N.H. vii. p. 106. D.

1854 „Pfr., Conch. Cab. p. 390. pl. 143 (1852), f. 16-18. D.F.

1853 ,"

Mon. Hel. iii. p. 93.

$\begin{array}{lll}\prime \prime & " & \\ 1856 & , & \text { Rve., Conch. Icon. pl.173, f.1172. D.F. }\end{array}$

1887 Helix (Pella) dumeticola, Bs., Tryon, Man. of Conch. iii.p. 106. pl. 20, f. 79. D.F.

1903 Rhytida (Macrocycloides) dumeticola, Bs., Mlldff., Conch. Cab. p. 59. pl. 10, f. 20-22. D.F.

Type in British Museum.

Hab. Cape Peninsula. Green Point; Simonstown; Simon's Bay (Benson). Camp's Bay (Layard). Hout Bay; Milnerton; Kommetje (Connolly); Robben Island (Fisk).

121. Natatina eumacta (Melv. \& Pons.).

1892 Helix (Aerope) eumacta, M. \& P., A.M.N.H. x. p. 237. pl. 13, f. 4 . D.F.

1903 Natalina eumacta, M. \& P., Mlldff., Conch. Cab. p. 22. pl. 3, f. $9 . \quad D . F$.

Type in British Museum.

Hab. Cape of Good Hope (not Natal). Bashee River, Idutywa (Crawford). 
122. Natalina inhluzana (Melv, \& Pons.).

1894 Helix (Dorcasia) inhluzana, M. \& P., A.M.N.H.xiv. p.91.pl.1, f. 4. D.F.

1895 Dorcasia inhlizana, M.\&P., A.M.N.H.xv. p. 165.pl.12, f.6. F. 1903 Rhytida (Afrorhytida) inhluziana, M. \& P., Mlldff., Conch. Cab. p. 64. pl. 11, f. 7. D.F.

1907 " inhluzana, M. \& P., A.M.N.H. xix. p.99. N.

'Type in British Museum.

Hab. Natal. Inhluzani Mountain (Mrs. Shaw).

"Nearly allied to, if not a variety of, $R$. kraussi, Pfr." (M. \& P., 1907).

123. Natalina insignis, Melv. \& Pons.

1907 Natalina insignis, M. \& P., A.M.N.H.xix. p. 98. pl. 6, f.9. D.F. Type in British Museum.

Hab. Cape of Good Hope. Teafontein, Grahamstown (Farquhar).

124. Natalina knysnaensis (Pfeiffer).

[S.A.M.]

1845 Helix knysnaensis, Pfr., P.Z.S. p. 131. D.

1846

1847

1848

1852

1853

,
" A.M.N.H. xvii. p. 439 . D.

"Phil., Abb. u. Beschr. ii. p. 85. pl. 7, f. 5. D.F.

„ Mon. Hel. i. p. 84. D.

" Rve., Conch. Icon. pl. 177, f. 403. D.F.

" Conch. Cab. p. 343. pl. 133 (1852), f. 5, 6. D.F.

1887 Helix (Pella) knysnaensis, Pfr., Tryon, Man. of Conch. iii. p. 106. pl. 20, f. 75. D.F.

1889 Aerope knysnaensis, Pfr., Pilsb., Proc. Acad. Nat. Sci. Phila. p. 277. pl. 9, f. A-G. F.A.R.

1890 " Pilsb., Proc. Acad. Nat. Sci. Phila. p. 41. N.

1903 Rhytida (Afrorhytida) knysnaensis, Pfr., Mlldff., Conch. Cab. p. 61. pl. 11, f. 1, 2. D.F.

Type in British Museum.

Hab. Cape of Good Hope. Knysna (coll. Cuming). Port Elizabeth (Crawford; Farquhar). Somerset East (Miss Bowker).

125. Natalina kraussi (Pfeiffer).

1846 Helix kraussi, Pfr., Symb. iii.p.70. D.

1848 " , " Mon. Hel. i. p. 197. D. 
1848 Helix kraussi, Pfr., Krs., Südafr. Moll. p. 77: pl. 4, f. 24. D.F. 1851 ", sturmiana, Pfr., P.Z.S. p. 253. D.

1853 ," , " , Mon. Hel. iii. p. 150. D.

" " " " $\quad$ Rve., Conch. Icon. pl.168, f. 1132. D.F.

1854 ." kraussi, Pfr., Rve., Conch. Icon. pl. 198, f. 1391. D.F.

" $\quad$ " sturmiana, Pfr., A.M.N.H. xiii. p. 142 . D.

, Conch. Cab. p. 397.pl. 144 (1852), f. 3ُ, 4. D.F.

1888 „, (Acusta) kraussi, Pfr., Tryon, Man. of Conch. iv. p. 50. pl. 10, f. 30. D.F.

1890 „, sturmiana, Pfr., Pilsb., Man. of Conch. vi. pl. 36, f. 28, 29. $F$.

1895 Rhytida kraussii, Pfr., Cooke, Camb. Nat. Hist., Moll. \& Brach. p. 232 , f. 139 . $R$.

1898 „, kraussi , (= sturmiana), M: \& P., Proc. Mal. Soc. iii. p. 170.

1903 ,, (Afrorhytida) kraussi, Pfr., Mlldff., Conch. Cab. p. 63. pl. 11, f. 5, 6. D.F.

Type of sturmiana in British Museum; kraussi-ubi?

Hab. Cape of Good Hope. Outeniqua, George District (Krauss). Knysna (fide Pfeiffer). Tharfield (Miss Bowker) and Grahamstown (Miss Glanville, fide Layard).

Orange Free State. Van Reenen, Drakensberg (Burnup).

Sturmiana was described from a shell in the Cuming collection,

"Hab. ?" but in the Nomenclator Pfeiffer gives the locality Delagoa Bay.

N. inhluzana (M. \& P.) is possibly a variety of kraussi.

126. Natalina lightfootiana, Melv. \& Pons. [S.A.M.]
[S. 1909 Natalina lightfootiana, M. \& P., A.M:N.H. iv. p. 490. pl. 8; f. $13 . D \cdot F$.

Type in British Museum.

Hab. Cape of Good Hope. Swellendam (Lightfoot). Montagu (Connolly).

As the shell of this species is not glossy, but dull, and the animal, to the best of my belief, not carnivorous, it probably belongs to a different family.

127. Natalina liliacea, Preston.

1912 Natalina liliacea, Prest., Proc. Mal. Soc. x. p. 17. D.F.

Type in coll. Dautzenberg.

Hab. Cape of Good Hope. Knysna Forest (Cox). 
128. Nataima Liparoxantha (Melv, and Pons.). [S.A.M.] 1892 Helix (MFacrocyclis) liparoxantha, MI. \& P., A.M.N.H. x. p. 238. pl. 13, f. 3. D.F.

1903 Rhytida (Macrocycloides) liparoxantha, M. \& P., Mlldff., Conch. Cab. p. 58. pl. 10, f. 18. D.F.

Type in British Museum.

Hab. NataL. Pietermaritzburg; Pinetown; Table Mountain; Port Shepstone (Burnup).

Zululand, Hlabisa (Burnup).

CAPE OF Good Hope. Transkei (Weisbecker).

Very closely allied to $N$ : coenotera (M. \& P.) and vernicosa (Krs.).

129. Natalina oconnori, Preston.

1912 Natalina oconnori, Prest., A.M.N.H. ix. p. 445. D.F.

Type in coll. Preston.

Hab. Cape of Good Hope. Ceres District (O'Connor).

130. Natalina Quekettiana (Melv. \& Pons.).

1893 Helix (Macrocyclis) quekettiana, MI. \& P., A.M.N.H. xii. p. 103. pl. 3, f. 1. D.F.

1903 Rhytida (Afrorhytida) queckettiana, M. \& P., Mlldff., Conch. Cab. p. 63. pl. 11, f. 4. D.F.

Type in British Museum.

Hab. Natal. Town Bush, Pietermaritzburg (Quekett).

131. Natalina schaerfie (Pfeiffer).

1861 Helix schaerfice, Pfr., Mal. Blätt. viii. p. 73. pl. 2, f. 1-3. D.F. 1864

1868 „ Bs., A.M.N.H. xiii. p. $494 . \quad N$.

1886

" Mon. Hel. v. p. $242 . \quad$ D.

" Kob., Conch. Cab. p. 615. pl. 178, f. 1-6. D.F.

1890 Helix (Ampelita) schaerfice, Pfr., Pilsb., Man. of Conch. vi. p. 43. pl. 7 , f. $95,96,97,1,2$. D.F.

Type in Stettin Museum.

Hab. Cape of Good Hope. Bredas Bosch, Gnadenthal District (Mrs. Schärf). Oudebosch; Bredasdorp (Layard).

132. Natalina tarachodes, sp. nov., pl, 2, f. 4. [S.A.M.]

Shell small, depressed, rounded, broadly and deeply umbilicate, very glossy, transparent, golden corneous. Spire not elevated. Whorls $4 \frac{1}{2}$, flattened above, but not carinated, rapidly increasing 
covered with very faint, regular, curved stric, which are still fainter on the under side. Suture simple, shallow. Aperture nearly circular, descending a little in front. Peristome thin, simple, receding sharply in profile from above. Columella very weak, concave, without marginal reflexion.

Diam. maj. 8 , min. 7 ; alt. 3.3 ; apert. alt. $3 \cdot 2$, lat. $3.7 \mathrm{~mm}$.

Radular formula $12+0+12 \times 35$.

Type in British Museum.

Hab. Cape Peninsula (generally distributed).

This little species has been known for fifty years, but has been generally misidentified as vernicosa, Krauss, or bullacea, Pfr., under one of which names it appears in many museum and private collections. It is a far smaller form than vernicosa, with the type of which I have compared it, while bullacea is an Australian species, non-existent in South Africa. N. vernicosa, var. minor, Pfr., from Natal, which I have not seen, may be near akin to tarachodes, but, if so, is worthy of specific rank.

133. Natalina trimeni (Melv. \& Pons.).

[S.A.M.]

1892 Helix (Aerope) trimeni, M. \& P., A.M.N.H. x. p. 237. pl. 13, f. 1. D.F.

1893 Natalina tremeni, M. \& P., Pilsb., Man. of Conch. viii. p. 135. (Err. typ.)

1895 " trimeni, M. \& P., Pace., Proc. Mal. Soc. i. p. 232. A.R. 1903 Rhytida (Afrorhytida) trimeni, M. \& P., Mlldff., Conch. Cab. p. 62. pl. 11, f. 3. D.F.

Type in British Museum.

Hab. "S. AFRICA" (Trimen).

CApe of Good Hope. Somerset East (Miss Bowker). Grahamstown; Cradock (Farquhar). Kowie, Port Alfred (Crawford). Witmoss (Reeve),

\section{Natalina vernicosa (Krauss).}

1848 Helix vernicosa, Krs., Südafr. Moll. p. 76. pl. 4, f. 23. D.F.

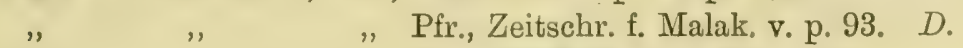

$\begin{array}{lllll}\prime \prime & " & " & \text { Prr., Zeitschr. f. Malak, v. p. 93. D. } \\ 1850 & " & \text { " } & \text { Bs., A.M.N.H. vi. p. 254. N. } & \\ 1851 & ", & \text { ", Rve., Conch. Icon. pl. 43, f. 198. D.F. } \\ 1853 & ", & \text { " } & \text { Pfr., Mon. Hel. iii. p. 95. D. }\end{array}$

1879 Rhytida vernicosa, Krs., Binn., Ann. N.Y. Acad. Sci. i. p. 355.

$$
\text { pl. 14, f. I. } R \text {. }
$$


1884 Rhytida vernicosa, Krs., Binn., Ann. N.Y. Acad. Sci. iii. p. 82. pl. 17, f. L. $R$.

1885 Elaa vernicosa, Krs., Tryon, Man. of Conch. i. p. 130. pl. 28, f. 52-54. D.F.

1903 Rhytida (Macrocycloides) vernicosa, Krs., Mlldff., Conch. Cab. p. 57. pl. 10, f. 12-14. D.F.

Type in Stuttgart Museum.

Hab. NataL (Wahlberg).

Cape of Good Hope. Port Elizabeth (fide Morelet). Grahamstown (Miss Glanville, tide Layard).

A good deal of confusion has existed with regard to this species, and it is by no means certain that some of the specimens described or figured by the foregoing authors, especially Binney and Möllendorff, are correctly identified.

The type appears to be an immature example, nearly akin to N. liparoxantha (M. \& P.).

var. MiNon, Pfeiffer.

1853 Helix vernicosa, Krs., var. minor, Pfr., Mon. Hel. iii. p. 95. D. 1885 Elaa " " " , Tryon, Man. of Conch. i. p. 131. N.

"var. $\beta$. Minor, costulis superficiei obsoletioribus" (Pfr., l.c.).

Hab. Natal (fide Pfeiffer).

135. Natalina viridescens (Melv. \& Pons.). : [S.A.M.] 1891 Helix (Patula) viridescens, M. \& P., A.M.N.H. viii. p. 238. D. $1892 \quad " \quad, \quad$, . , ix. pl. 4,f. 1. F. " , $\quad$, $\quad$, Pilsb., Man. of Conch. viii. p. 78. pl. 30, f. 32. D.F. 1903 Rhytida (Macrocycloides) viridescens, M. \& P., Mlldff., Conch. Cab. p. 61. pl. 10, f. 26 . D.F.

Type in British Museum.

Hab. Transvaal. Pretoria (Farquhar; MeBean).

NataL. Dargle; Inhluzani (Burnup).

Tribe HOLOGNATHA; W. G. Binney, 1878.

(Moll. United States, v. p. 81.)

FAmily ZONITID无, Mörch, 1863.

(Vid. Med. naturhist. Foren. Copenhagen, p. 267.) 
Sub-Family HELICARIONIN E, Godwin-Austen, 1883. (Moll. of India, pt. 4, p. 146.)

Genus ZINGIS, von Martens, 1878. (Monats-Ber. K. Akad. Wiss. Berlin, p. 290.) (=Sheldonia, Ancey, 1888.)

Type of Genus, Z. ractiolata, von Mts.

136. Zingis Crawfordi (Melv. \& Pons.). 1890 Helix (Pella) crawfordi, M. \& P., A.M.N.H. vi. p. 469. D.

$1892 \quad$ " " " ix.p.94.pl.4,f.4. $F$. 1893 Phasis (Trachycystis) cranfordi, MI. \& P., Pilsb., Man. of Conch. viii. p. 146. pl. 35 , f. 10. D.F.

Type in British Museum.

Hab. CAPE of Good Hope. Middleton; Kleinpoort, Graff Reinet line (Crawford). Cradock (Farquhar). Witmoss (Reeve). Somerset East (in S.A. Museum).

137. Zingis delicata, Melv. \& Pons.

1895 Z ingis delicata, M. \& P., A.M.N.H.xv. p. 163. pl. 12, f. 1. D.F.

Type in British Museum.

Hab. Cape of Good Hope. Knysna District (Cox; O'Connor; Purcell).

138. Zingis haygarthi, Melv. \& Pons.

1899 Zingis haygarthi, M. \& P., A.M.N.H. iv. p. 195. pl. 3, f. 3. D.F. Type in British Museum.

Hab. ZuluLand. 'Nkhandla Forest (Haygarth).

139. Zingis minythodes (Melv. \& Pons.). [S.A.M.]

1892 Helix (Pella) minythodes, M. \& P., A.M.N.H. x. p. 240. pl. 13, f. 8. D.F.

1893 Phasis (Trachycystis) minythodes, M. \& P., Pilsb., Man. of Conch. viii. p. 144. pl. 35, f. 15 . D.F.

Type in British Museum.

Hab. CaPe of Good Hope. Craigie Burn, Somerset East (Crawford).

140. Zingis perlevis, Preston.

1912 Zingis perlevis, Prest., Proc. Mal. Soc. x. p. 17. D.F.

Type in coll. Dautzenberg.

Hab. Cape of Good Hope. Knysna Forest (Cox).

Possibly better placed in Peltatina. 


\section{Zingis rosenbergi, Preston.}

1909 Zingis rosenbergi, Prest., A.M.N.H. iv. p. 498. D.F.

Type in British Museum.

Hab. Transvaal. Pietersburg (fide Preston).

142. Zingis thermarum, Melv. \& Pons.

1909 Zingis thermanum, M. \& P., A.M.N.H. iv. p. 491. pl. 8, f. 14. D.F.

Type in British Museum.

Hab. Transvaal. Warmbaths; Pietpotgietersrust (Connolly).

Rhodesia. Umtali (in S.A. Museum).

Genus HeLiCARION, Férussac, 1821.

(Trabl. Syst. Moll. pt. 3, p. 23 (or 19), as Helixarion, emend. p. 71 (or 67).

Type of Genus, H. cuvieri, Fér.

Most of the following species should doubtless be placed in Peltatince, but, without knowledge of the animal, it is impossible to determine their exact generic position.

143. Helicarion chrysoprasinus (Melv. \& Pons.).*

1892 Vitrina chrysoprasina, M.\& P., A.M.N.H.x.p.241.pl.13, f.11. D.F.

Type in British Museum.

Hab. CAPE of Good Hope. Cathcart (ex coll. Wotton).

Orange Free State. Thaba N'chu (Connolly).

The locality-Pretoria-given for the type is almost undoubtedly erroneous.

144. Helicarion coxi, Preston.

1912 Helicarion coxi, Prest., Proc. Mal. Soc. x. p. 16. D.F.

Type in coll. Dautzenberg.

Hab. Cape of Good Hope. Knysna (Cox).

145. Helicarion knysnaensis, Preston.

1912 Helicarion knysnaensis, Prest., Proc. Mal. Soc. x. p. 16. D.F. Type in coll. Dautzenberg.

Hab. Cape of Good Hope. Knysna (Cox).

146. Helicarion planti (Pfeiffer).

1856 Vitrina planti, Pfr., P.Z.S., p. 324. D.

$1859 \quad$ " $\quad$ Mon. Hel, iv. p. 794. D.

$1862 \quad " \quad$ " Rve., Conch. Icon. pl. 8, f. 57. D.F.

1885 Vitrina (Gallandia) planti,Pfr., Tryon, Man. of Conch.i.p.157. D.

* Since the above was printed, this Species has been proved to belong to Microkerkus. 
Reference List of South African Non-marine Mollusca. 101

Type in British Museum.

Hab. Natal (Plant).

A very distinct species, perhaps more nearly akin to true Helicarion than others of the present group.

147. Helicarion pumilio, Melv. \& Pons. *

1909 Helicarion pumilio, M.\& P., A.M.N.H. iv. p. 490. pl. 8, f. 11. D.F. Type in British Museum.

Hab. Transvaal. Zoutpansberg (Cregoe).

148. Helicarion russofulgens, Melv. \& Pons.

1909 Helicarion russofulgens, M.\&P.,A.M.N.H.iv.p.490.pl.8,f.12. D.F.

Type in British Museum.

Hab. Zululand. Eshowe (Lady Saunders).

Natal. Tongaat (Burnup). ? Hilton Road (fide M. \& P.)

149. Helicarion transvaalensis (Craven).

1880 Vitrina transvaalensis, Crvn., P.Z.S. p. 615. pl. 57, f. 3. D.F. 1885 " (Gallandia) transvaalensis, Crvn., Tryon, Man. of Conch. i. p. 156. pl. 34 , f. 88,89 . D.F.

1912 "New Genus?" transvaalensis, Crvn., G.-Aust., A.M.N.H. ix. p. 128.

Type in British Museum.

Hab. Transvaal. Lydenburg (Craven).

Natal. Majuba (Connolly).

Basutoland. Mont-aux-Sources (Warren).

150. Helicarion vandenbroecki (Craven).

1880 Vitrina vandenbroeckii, Crvn., P.Z.S. p. 615. pl. 57, f.4. D.F. 1885 " (Gallandia) vandenbroeckii, Crvn., Tryon, Man. of Conch. i. p. 156. pl. 34, f. 90,91 . D.F.

Type in British Museum.

Hab. Natal. Lydenburg (Craven).

\section{SUB-FAMILX TROCHONANININ 王 (nov.).}

Genus MARTENSIA, Semper, 1870.

(Reis. im Archip. Philippin., ii, 3. p. 42.) (=Ledoulxia, Bgt., 1885, par's.)

Type of Genus, M. mozambicensis (Pfr.).

* This Species has just been constituted Type of the new Genus Andrarion, Godwin-Austen. 
151. Martensia mozambicensis (Pfeiffer).

1855 Helix mozambicensis, Pfr., P.Z.S. p. 91. pl. 31, f. 9. D.F.

1859

1869

" . " Mon. Hel. iv. p. 32. D.

" , Novit. Conch. iii. p. 499. pl. 108, f. 1-3. D.F.

1870 ILartensia mossambicensis, Pfr., Semp., Reis. Archip. Philippin. ii, 3. p. 42. pl. 3, f. 5. pl. 6, f. 15. A.R.

1874 ", mozambicensis, Pfr., Jick., Fauna N.-O.-Afr. p. 49. D.

1881 Helix mozambicensis, Pfr., Dohrn, Conch. Cab. p. 609. pl. 177,

f. 7-11. D.F.

1883 Trochomorpha mozambica, Bgt., Ann. Sci. Nat. Paris, xv.p.107. L.

1885 Trochonanina mozambicensis, Pfr., Bgt., Hélixarionidées, p. 7. N.

1886 Nanina (Martensia) mozambicensis, Pfr., Tryon, Man. of Conch.

ii. p. 50. pl. 24 , f. 80 . D.F.

1889 Trochonanina mozambicensis, Pfr., Bgt., Moll. de l'Afr. équat. p. 17. $N$.

1894 "Smith., Proc. Mal. Soc. i. p. $164 . N$.

1895 Martensia mozambicensis, Pfr., G.-Aust., Proc. Mal. Soc. i. p. 281. pl. 19, f. 1. $A$.

1897 Trochonanina (Martensia) mossambicensis, Pfr., von Mts., D.-O.-Afr. p. 46. pl. 1, f. 8. D.F. p. 296. N.

1907 Martensia mossambicensis, Pfr., Melv. \& Standen, Manchester Memoirs, li, 4. p. $9 . \quad N$.

Type in British Museum.

Hab. Lorenzo Marques. Tette (Peters). Movene (Penther).

Transvaau. Barberton (Fry). Zoutpansberg (Cregoe).

RноDesia. Salisbury (Miss Weineck).

Widely distributed in East Africa.

von Martens (1897) considers Trochonanina livingstoniana, Ancey, from Nyassa, to be merely a small, high form of this species.

var. АLворістA, von Martens.

1869 Nanina mossambicensis, Pfr., var. albopicta, von Mts., von der Decken's Reisen in Ostafrica, iii. p. 56. pl. 1, f. 2. D.F.

1878 Trochonanina mossambicensis, Pfr., var. albopicta, von Mts., Monats-Ber. Akad. Wiss. Berlin, p. 289. N. $1885 \quad$ " anceyi, Bgt., Helixarionidées, p. 9. D.

"Ledoulxia albopicta, von Mts., Bgt., Helixarionidées, p. 12. N. 1886 Martensia mozambicensis, Pfr., var. albopicta, von Mts., Tryon, Man, of Conch. ii. p. 50. N. 
1889 Trochonanina anceyi, Bgt., Moll. de l'Afr. équat. p. 20. D. 1897 mossambicensis, Pfr., var. albopicta, von Mts., D.-O.-Afr. p. 47. D.

Hab. Mozambique. Tette (Peters).

Occurring, often in company with the normal form, in many parts of East Africa.

var. ELATIOR, von Martens.

1866 Trochomorpha? mossambicensis, Pfr., var. elatior, von Mts., Mal. Blätt. xiii. p. 92 . D.

1869 Helix mozambicensis, var, elatior, von Mts., Pfr., Novit. Conch. iii. p. 500. pl. 108, f. 4-6. D.F.

1874 Martensia mozambicensis, Pfr., var. elatior, von Mts., Jick., Fauna N.-O.-Afr. p. 50. N.

1897 Trochonanina mossambicensis, Pfr., var. elatior, von Mts., D.-O.-Afr. p. 47. pl. 3, f. 9. D.F.

Hab. Lorenzo Marques. Ikchongove (? Itschongove), (Schenck).

Not so plentiful, but rather more widely distributed than the normal form, occurring as far north as Abyssinia (von Martens).

Genus TROCHOMORPHA, Albers, 1850.

(Die Helic., p. 116.)

Type of Genus, T. trochiformis (Fér.).

152. Trochomorpha placenta, Melv. \& Pons.

1899 Trochomorpha placenta, M.\&P., A.M.N.H.iv.p.197.pl.3,f.9. D.F.

Type in British Museum.

Hab. Zululand. 'Nkandhla Forest (Haygarth).

Genus TROCHOZONITES, Pfeffer, 1883.

(Abhandl. Naturwiss. Verein. Hamburg, vii, 2. p. 23.)

Type of Genus, T. percarinatus (von Mts.).

153. Trochozonites dioryx, Melv. \& Pons.

1892 Helix (Trochozonites) dioryx, M. \& P., A.M.N.H. ix. p. 89. pl. 5, f. 2. D.F.

Type in British Museum.

Hab. Cape of Good Hope. Robbe Bay, Port Nolloth (Layard). 
Genus THAPSIELLA, Gude, 1911.

(Proc. Mal. Soc. ix. p. 272.)

Type of Genus, T. masakuensis (Smith).

154. Thapsiella connolityi, Preston.

1912 Thapsiella connollyi, Prest., A.M.N.H. ix. p. 70, f. 3. D.F. Type in coll. Preston.

Hab. RHodesia. Victoria Falls (Connolly).

Sub-Family PELTATIN Æ, Godwin-Austen, 1912. (A.M.N.H. ix. pp. 124, 126.)

Genus Peltatus, Godwin-Austen, 1908.

(A.M.N.H. i. p. 131.)

Type of Genus, $P$. aloicola (M. \& P.).

155. Peltatus aloicola (Melvo \& Pons.).

1890 Vitrina huttonia, Bs., var. aloicola, M.\&P., A.M.N.H.vi.p.467. D. 1908 Peltatus hudsonia, Bs., G.-Aust., A.M.N.H. i. p. 131.pl. 8, f.1. D.F.A.

1912 " aloicola, M. \& P., G.-Aust., A.M.N.H. ix. p. 130. pl. 4, f. 1. D.A.R.

Type in British Museum.

Hab. CAPE of Good Hope. Port Elizabeth, North End and Rufane Vale, living on aloes (Crawford).

156. Peltatus arnotiti (Benson).

1864 Helix arnotti, Bs., A.M.N.H. xiii. p. 491. D.

$1868 \quad$ " "Pfr., Mon. Hel. v. p. 67. D.

1909 Zingis annotti " M.\& P., A.M.N.H.iv. p. 491.pl. 8, f. 16. N.F.

1912 Peltatus " „ G.-Aust., A.M.N.H. ix. p. 137. N.

Type in British Museum.

Hab. Cape of Good Hope. Colesberg (Arnott).

157. Peltatus asthenes (Melv. \& Pons.).

1907 Helicarion asthenes, M.\& P., A.M.N.H.xix.p. 99.pl. 6, f. 10. D.F. Type in British Museum.

Hab. CAPE OF Good Hope. Cradock (Farquhar). 
158. Peltatus caledonensis, Godwin-Austen.

1912 Peltatus caledonensis, G.-Aust., A.M.N.H. ix. p. 132. pl. 2, f. 1. pl. 4, f. 3. pl. 5, f. 1. D.A.R.

Type in British Museum.

Hab. Cape of Good Hope. Houw Hoek (Connolly).

$$
\text { 159. Peltatus capsula (Benson). }
$$

1864 Helix capsula, Bs., A.M.N.H. xiii. p. 492. D.

$1868 \quad " \quad$ "Pfr., Mon. Hel, v. p. 52. D.

1892 Nanina hypochlora, M. \& P., A.M.N.H. ix. p. 87. pl. 4, f. 8. D.F. 1912 Peltatus capsula,Bs., G.-Aust., A.M.N.H.ix. p.133.pl.7, f.2. D.A.

Type of capsula in University Museum of Zoology, Cambridge; hypochlora in British Museum.

Hab. Cape Peninsula. Simonstown (Layard; Connolly).

Hypochlora was described from "Cape of Good Hope (Layard)"; Colonel Godwin-Austen has assisted me to compare the type with capsula, and agrees that the two species are inseparable.

$$
\text { 160. Peltatus cotyledonis (Benson). }
$$

1850 Helix cotyledonis, Bs., A.M.N.H. v. p. 216. D.

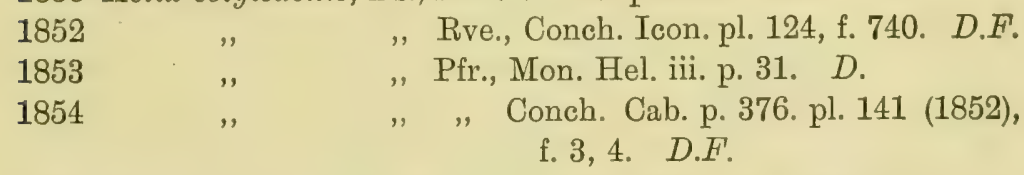

1887 Helix (Pella) cotyledonis, Bs., Tryon, Man. of Conch. iii. p. 103. pl. 20, f. 59. D.F.

1912 Peltatus cotyledonis, Bs., G.-Aust., A.M.N.H. ix. p. 134. A.R.

Specimens ex auct. in University Museum of Zoology, Cambridge.

Hab. CAPE of Good Hope. Simonstown (Benson). Kalk Bay (Layard). Fish Hoek; Hermanus; Saldanha Bay and Stumpnose, Malmesbury District (Lightfoot). Kommetje ; Buffelsfontein, near Cape Point (Connolly).

161. Peltatus hudsone (Benson).

1864 Helix hudsonia, Bs., A.M.N.H. xiii. p. 493. D.

$1868 \quad$ " "Pfr., Mon. Hel. v. p. 104. D.

1890 Vitrina huttonia " M. \& P., A.M.N.H. vi. pp. 467, 468. N. (Err. typ.)

1893 Pella (Gallandia) Inudsonie, Bs., Pilsb., Man. of Conch. viii. p. $135 . N$. 
1912 Helix hudsonia, Bs., G.-Aust., A.M.N.H. ix. p. 129. D.N. Probable type in British Museum.

Hab. Cape of Good Hope. Riversdale (Mrs. Hudson).

var. Rufofilosus, Melv. \& Pons.

1890 Vitrina huttonice,Bs.,var.,ufofilosa, M.\& P.,A.M.N.H.vi.p.467. D.

Hab. CAPE of Good Hope. Rufane Vale, Port Elizabeth (Crawford).

var. meridionalis, Melv. \& Pons.

1890 Vitrina huttonia, Bs., var. meridionalis, M. \& P., A.M.N.H. vi. p. 467. D.

Hab. CAPE OF Good Hope. Port Elizabeth, North end (Crawford).

It is by no means certain whether these two varieties should be referred to hudsonce or to aloicola, M. \& P.

\section{Peltatus natalensis (Pfeiffer).}

1846 Helix natalensis, Pfr., Symb. iii. p. 65. D.

1848

1849

1854

1887 Helix (Pella) natalensis, Pfr., Tryon, Man. of Conch. iii. p. 103. pl. 20 , f. 58. D.F.

1889 Ariophanta (Zingis) natalensis, Pfr., Pilsb., Proc. Acad. Nat. Sci. Phila. p. 279. pl. 9, f. H, I. A.R.

1912 Peltatus natalensis, Pfr., G.-Aust., A.M.N.H. ix. p. 136. pl. 3, f. 2. pl. 6, f. 2. D.F.A.R.

Type in Stettin Museum.

Hab. Natal. Port Natal (Menke).

CAPE of Good Hope. Port Elizabeth (Crawford; Farquhar).

163. Peltatus pellicula (Férussac).

1821 Helix (Helicolimax) pellicula, Fér., Tabl. Syst. Moll. pt. 3. p. 25 (or 21). L.

1842 Vitrina pellicula, Fér., Pfr., Symb. ii. p. 17. D.

1848

1851

1862

1885 Vitrina "(Gallandia) pellicula, Fér., Tryon, Man. of Conch. 
Type $u b i$ ?

Hab. Cape of Good Hope. "On aloes" (Delalande). Port Elizabeth (fide Crawford).

Not the Helix pellicula, Fér., of Pfr., 1851 (Conch. Cab. p. 47), nor of Desh., 1850 (Hist. nat. Moll. i. p. 204), which is trifasciate, and apparently a West Indian species. The true pellicula would appear to be a Peltatus, possibly identical with aloicola.

\section{Peltatus phytostylus (Benson).}

1864 Helix phytostylus, Bs., A.M.N.H. xiii. p. $492 . \quad D$.

$1868 \quad$ " " Pfr., Mon. Hel. v. p. 52. D.

1912 Peltatus phytostylus,Bs., G.-Aust., A.M.N.H.ix.pp.127, 137. N.

Type in British Museum.

Hab. Cape of Good Hope. Colesberg (Arnott). Riversdale, Swellendam District (Mrs. Hudson). Port Elizabeth (Crawford).

165. Peltatus trotterianus (Benson).

[S.A.M.]

1848 Helix trotteriana, Bs., A.M.N.H. ii. p. 161. D.

1853

" Rve., Conch. Icon. pl.124, f. 745. D.F.

1854

,, Pfr., Mon. Hel. iii. p. 29. D.

," , , Conch. Cab. p. 388. pl. 143 (1852), f. 1, 2. D.F.

1887 Helix (Pella) trotteriana, Bs., Tryon, Man. of Conch. iii.p. 103. pl. 20, f. 57. D.F.

1912 Peltatus trotteriana, Bs., G.-Aust., A.M.N.H. ix. p. 135. pl. 5, f. 2. pl. 6 , f. 1. D.A.R.

Type in British Museum.

Hab. Cape of Good Hope. Uitenhage (Trotter). Port Elizabeth (Crawford). Knysna (Purcell). Cradock (Farquhar). Bokkeveldt (Layard).

Basutoland. Maseru (Sclater).

Genus KERKOPHORUS, Godwin-Austen, 1912 .

(A.M.N.H. ix. p. 127.)

Type of Genus, $K$. imunctus (M. \& P.).

166. Kerkophorus ampliatus (Melv. \& Pons.).

1899 Zingis ampliata, M. \& P., A.M.N.H. iv. p. 196. pl. 3, f. 5. D.F.

Type in British Museum.

Hab, Natal, Durban (Burnup).

Apparently near akin to natalcnsis, Krs. 
167. Kerkophorus cingulatus (Melv. \& Pons.). [S.A.M.] 1890 Vitrina cingulata, M. \& P., A.M.N.H. vi. p. $466 . \quad D$.

1892 " ix. p. 94. pl. 5, f. 8. F.

Type in British Museum.

Hab. Cape of Good Hope. Bellevue; Tootabi, near Alicedale; Alexandria District (Crawford). Bedford (Farquhar). Port St. John's (Shortridge). Kentani (Miss Pegler).

It is questionable whether this species is separable from the Natalian poeppigi, Menke, which it much resembles.

168. Kerkophorus corneus (Pfeiffer).

1846 Vitrina cornea, Pfr., Symb. iii. p. 81. $D$.

$1848 \quad$ " " Krs., Südafr. Moll. p. 74. N.

" $\quad$ " Mon. Hel. ii. p. 505. D.

$1854 \quad$ " $\quad$ "Conch. Cab. p. 21. pl. 2, f. 31-33. D.F.

$1862 \quad$ " "Rve., Conch. Icon. pl. 4, f. 24. D.F.

1885 Vitrina (Gallandia) cornea, Pfr., Tryon, Man. of Conch. i. p. 157. pl. 34, f. 92-94. D.F.

1912 Kerkophorus comens? Pfr., G.-Aust., A.M.N.H. ix. p. 137. pl. 1, f. 1 . $A$.

Type in Stettin Museum.

Hab. Natal. Port Natal (Menke).

CAPe of Good Hope. Eastern Province (Krauss). Port Elizabeth (fide Morelet).

169. Kerkophorus fuscicolor (Melv. \& Pons.). [S.A.M.]

1892 Vitrina fuscicolor, M. \& P., A.M.N.H. x. p. 240. pl. 13, f. 10. D.F. 1903 Natalina ", " Mlldff., Conch. Cab. p. 23. pl. 3, f. 10 . D.F.

Type in British Museum.

Hab. Orange Free State. Rensberg's Kop, Drakensberg (Quekett). Platberg, Harrismith (Connolly).

170. Kerhophorus inunctus (Melv, \& Pons.). [S.A.M.] 1860 Helix congellana, Krs., von Mts., Die Helic. p. 84. L.

1899 Zingis inuncta, M. \& P., A.M.N.H. iv. p. 195. pl. 3, f. 4. D.F. 1912 Kerkophorus inunctus, M. \& P., G.-Aust., A.M.N.H. ix. pp. 127, 138. pl. 3, f. 1. F.A.

Type of inunctus in British Museum; congellana in Stuttgart Museum. 
Reference List of South African Non-marine Mollusca. 109

Hab. Natal. Umkomaas; Alexandra Junction (Burnup). Congella (congellana, Wahlberg).

Zululand. 'Nkandhla Forest (Haygarth). Umbonambi (Toppin). Makowe (Crosly).

Through the kindness of Dr. Lampert, of Stuttgart, I have been enabled to examine the type of the little-known $H$. congellana. It is identical with inunctus, but, never having been described or figured, must yield precedence to the last-named species.

171. Kerkophorus leucospira (Pfeiffer).

1856 Vitrina leucospira, Pfr., P.Z.S. p. 326. D.

[S.A.M.] 1862 " Rve., Conch. Icon. pl. 6, f. 42. D.F. 1885 Helicarion leucospirus, Pfr., Tryon, Man. of Conch. i. p. 168. pl. 28, f. 39. D.F.

1899 " leucospira, Pfr., M. \& P., A.M.N.H. iv. p. 192. N.

Type in British Museum.

Hab. Natal. Pinetown; Tongaat; Malvern (Burnup). Umgeni Rivermouth (Quekett).

Described as from South Australia.

\section{Kerkophorus melvilit, Godwin-Austen.}

1912 Kerkophorus melvilli, G.-Aust., A.M.N.H.ix.p.127.pl.7,f.1. A.R. Type in British Museum.

Hab. NataL. Equeefa (Burnup).

173. Kerkophorus natalensis (Krauss).

1848 Vitrina natalensis, Krs., Südafr. Moll. p. 74. pl. 4, f. 17. D.F.

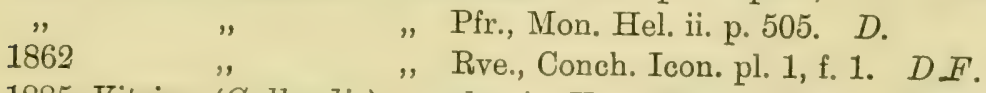

1885 Vitrina (Gallandia) natalensis, Krs., Tryon, Man. of Conch. i. p. 156. pl. 34 , f. 83,84 . D.F.

1909 Helicarion subcomea, Prest., A.M.N.H. iv. p. 498. D.F.

Type of natalensis in Stuttgart Museum; subcornea in British Museum.

Hab. NataL. Natal Bay (Krauss).

Cape of Good Hope. Somerset East and Tharfield (Miss Bowker), and Hillside, Grahamstown (Miss Glanville), fide Layard.

Subcomea was described from "Natal"; it appears quite inseparable from natalensis, Krs. Ampliatus and zonamydrus, M. \& P., are also very similar to the last-mentioned shell. 
174. Kerkophorus Ph玉dinus (Melv. \& Pons.). [S.A.M.]
[S. 1892 Vitrina phadima, M. \& P., A.M.N.H. x. p. 241. pl. 13, f. 12. D.F. $1898 \quad$, " , Stur., S.A. Moll. p. 34. N.

1912 Kertophorus phactimus, M.\& P., G.-Aust., A.M.N.H. ix. p. 139. pl. 5, f. $3 . A$.

Type in British Museum.

Hab. Natal. Pietermaritzburg; Durban; Pinetown; Dargle; Karkloof ; Port Shepstone; Ntimbankulu (Burnup).

Cape of Good Hope. Grahamstown; Bedford (Farquhar). Pirie Mountain (Godfrey).

175. Kerkophorus poeppigr (Menke).

1846 Vitrina poeppigii, Mke., Pfr., Symb. iii. p. 81. D.

1848 ", Krs., Südafr. Moll. p. 74. N.

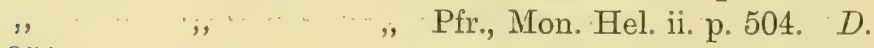

$1854 \quad$ " $\quad$ " " Conch.Cab.p.17.pl.2,f.13-15. D.F.

1862 ". " Rve., Conch. Icon. pl. 7, f. 49. D.F.

1885 Vitrina (Gallandia) poeppienii, Mke., Tryon, Man. of Conch.

i. p. 156. pl. 34 , f. 85-87: D.F.

Type in Stettin Museum.

Hab. Natal. Port Natal (Menke). On plants, widely distributed (Krauss).

Tränsvaat. "Moori" (Mooi) River (Craven).

Lorenzo Marques. Rikatla (Junod).

176. Kerkophorus vitalis (Melv. \& Pons.). [S.A.M.]
[S.M. 1908 Helicarion vitalis, M. \& P., A.M.N.H. i. p. 133. pl. 7, f. 4. D.F. 1912 Kerkophorus ", , $\quad$ G.-Aust., A.M.N.H. ix. p. 138.pl. 3, f. 3 . A.

Type in British Museum.

Hab. 'Natal. Port Shepstone; Durban (Burnup).

177. Kerkophorus zonamydrus (Melv.\& Pons.). [S.A.M.] 1890 Vitrina zonamydra, M. \& P., A.M.N.H. vi. p. 467. D.

$1892 \quad$ " $\quad$, $\quad$ ix. p. 94. pl. 5, f. 9. F.

Type in British Museum.

Hab. Cape of Good Hope. Alexandria District (Crawford). Kowie (Farquhar).

Natal. Durban (Penther, fide Sturany). 
Genus MICROKERKUS, Godwin-Austen, 1912.

(A.MI.N.H. ix. p. 128.)

Type of Genus, $M$. symmetricus (Crvn.).

178. Microkerkus pondoensis, Godwin-Austen. [S.A.M.] 1912 Microkerkus pontoensis, G.-Aust., A.M.N.H. ix. p. 128. pl. 4, f. 2 . A.R.

Type in British Museum.

Hab. Cape of Good Hope. Kentani (Niss Pegler).

179. Microkerkus stmietricus (Craven).

1880 Helix symmetrica, Crvn., P.Z.S. p. 614. pl. 57, f. 2. D.F. 1887 ", (Pella) symmetrica, Crvn., Tryon, Man. of Conch. iii. p. 108. pl. 21, f. 99 . D.F.

1898 Pella symmetrica, Crvn., Stur., S.A. Moll. pp. 39, 40. N. 1912 Microkerkus symmetricus, Crvn., G.-Aust., A.M.N.H. ix. pp. 128, 137. pl. 1, f. 2. pl. 3, f. 4. F.A.

Type in British Museum.

Hab. Transvalu. Lydenburg (Craven). Pretoria (McBean).

\section{Sub-Family ZONITINæe, Pilsbry, 1898.}

(Nautilus, xi. p. 128.)

Genus VITREA; Fitzinger; 1833.

(Beiträge zur Landeskunde Oesterreichs u.d. Enns, Wien, iii. p. 99.)

(= Hyalinia, Charpentier, 1837, nec Sehumacher, 1817.)

Type of Genus, V. diaphana (Studer).

180. Vitrea crxstallina (Müller).

1774 Helix crystallina, Müll., Verm. ii. p. 23. D.

1805 " cristallina ", Drap., Hist. Moll. Fr. p. 118. pl. 8, f. 13-18. D.F.

1821 " eburnea, Hartm., Neue Alp. i..p. 234.

" $\quad$ " crystallina, Müll., C. Pfr., Syst. Anordn. deutsch. L.-u. W. Schn. p. 46. pl. 2, f. 36. D.F.

1829 " vitrea, Brown, Edinb. Journ. Nat. \& Geogr. Sci. i. p. 12. pl. 1, f. 12-14. D.F.

1830 „ crystallina, Müll. (=vitrea, Brown), Alder, Cat. Newcastle, p. 11. D. 
1840 Zonites crystallinus, Müll., Gray, Turton's Man. p. 176. pl. 4, f. 42 . D.F.

1848 Helix crystallina, Müll., Pfr., Mon. Hel. i. p. 59. D.

1852

, Pfr., Conch. Cab. p. 128. pl. 88 (1850), f. 27-30. D.F.

1855

" Moq.-Tand., Hist. Nat. Moll. Fr. ii. p. 89. pl. 9, f. 26-29. D.F.

1856 Zonites crystallinus „, Bgt., Amén. malac. i. p. 192. pl. 20, f. 19-24. D.F.

1862 " " , Jeffr., Brit. Conch. i. p. 170. and v. (1869) pl. 10, f. 3. D.F.

1864 " enstilbus, Bgt., Mal. Algérie, i. p. 76. pl. 4, f. 11-16. D.F. 1881 Hyalina cavanna, Paulucci, Bull. Soc. Mal. Ital. p. 80. pl. 1 bis, f. 3. D.F.

1886 Hyalinia (Vitrea) crystallina, Müll., Tryon, Man. of Conch. ii. p. 138. pl.46, f. 3-8. D.F.

1908 Tayl., Mon. Brit. Moll. Type-ubi? p. 108. pl. 2 and pl. 15 (1912). D.F.A.R.

Hab. Cape Peninsula. Cape Town and suburbs (plentiful). A common European species.

Genus POLITA, Held, 1837.

(Isis, p. 916.)

Type of Genus, $P$. cellaria (Müll.).

181. Polita alliaria (Miller).

1822 Helix alliaria, Mill., Ann. Philos. iii. p. 379. D.

1828 " fœtida, Stark, Elem. Nat. Hist. ii. p. 59. D.

1833 " alliacea; Jeffr., Linn. Trans. xvi. p. 341. D.

1840 Zonites alliarius, Mill., Gray, Turton's Manual, p. 168. pl. 4, f. 39 . D.F.

1851 Helix remota, Bs., A.M.N.H. vii. p. 263. D.

1854 Zonites alliarius, Mill., Schm., Mal. Blätt. i. p. 8. N.

1855

" Moq.-Tand., Hist. Nat. Moll. Fr. ii. p. 83. pl. 9, f. 9-11. D.F.

1859 Helix alliaria, Mill., Pfr., Mon. Hel. iv. p. 76. D.

1862 Zonites alliarius, Mill., Jeffr., Brit. Conch. i. p. 161. and v. (1869), pl. 9, f. 2. D.F.

1886 Hyalinia (Polita) alliaria, Mill., Tryon, Man. of Conch. ii. p. 145. pl. 47, f. 91-93. D.F. 
1907, 8 Hyalinia (Euhyalinia) alliaria, Mill., Tayl., Mon. Brit. Moll. p. 57. pl. 2 (1908), pl. 6 (1909). D.F.A.R.

Type of remota in British Museum ; alliaria-ubi?

Hab. CaPe of Good Hope. Grahamstown (Farquhar).

A common European species, probably of recent importation into South Africa.

182. Poltwa celuaria (Müller).

[S.A.M.]

1774 Helix cellaria, Müll., Verm. ii. p. 28. D.

$1786 \quad$ " .. $\quad$, Chem., Conch. Cab. ix, 2. p. 102. pl. 127, f. 1129. D.F.

$1788 \quad$ " $\quad$," $\quad$ ", Gmel., Syst. Nat., Ed. 13. i. p. 3634. D. 1803 ", lucida, Mont., Test. Brit. p. 425 ; and Suppl. (1808), pl. 23, f. 4. D.F.

1807 " nitens, Gmel., Mat. \& Rack., Linn. Trans. viii. p. 198. pl. 5, f. 7. D.F.

1817 " glaphyra, Say, Nicholson's Encycl. iv. pl. 1, f. 3. D.F.

1821 „, cellaria, Müll., C. Pfr., Syst. Anordn. deutsch. L.-u. W. Schn. p. 42. pl. 2, f, 29, 30. D.F.

$1822 \quad$ " $\quad$, $\quad$ " Lam., Hist. nat. An. s. Vert.vi, 2. p.91. D.

1830 " , (=nitida, Drap.), Alder, Cat. Newcastle, p. 12. D.

1840 Zonites cellarius, Müll., Gray, Turton's Manual, p. 170. pl. 4, f. 40 . D.F.

1841 Helix cellaria, Müll. (=glaphyra, Say.), A. Binn., Boston Journ. Nat. Hist. iii. p. 421. D.

1842 ", glaphyra, Say, Pfr., Symb. ii. p. 29. D.

1848 ", cellaria, Müll., Pfr., Mon. Hel. i. p. 111. D.

$1850 \quad$ " $\quad$ " $\quad$ Bs., A.M.N.H. v. p. 217. L.

1852 , " , " Pfr., Conch. Cab. p. 102. pl. 84 (1850), f. 8-10. D.F.

1855 Zonites cellarius, Müll., Moq.-Tand., Hist. Nat. Moll. Fr. ii. p. 78. pl. 9, f. 1, 2. D.F.

1862 (=lucida, Pult., nec Drap.), Jeffr., Brit. Conch. i. p. $159 . \quad D$.

1864 Helix sydneyensis, Cox, Cat. Austral. Land Shells, p. 37. D. 1868 " Mon. Austral. Land Shells, p. 9. pl. 9, f. 16. pl. 18 , f. 3. D.F.

1886 Hyalinia (Polita) cellaria, Müll., Tryon, Man. of Conch. ii. p. 155. pl. 50, f. 33-52. D.F.

1907 " (Euhyalinia) cellaria, Müll., Tayl., Mon. Brit. Moll. p. 30. pl. 2 (1908), pl. 6 (1909). D.F.A.R. 
1910 Hyalinia (Polita) cellaria, Müll., Bttgr., Abh. Senckenb. Naturf.

Ges. Frankfurt, xxxii. p. 455. N.

Specimens ex coll. Müller in University Zool. Mus. Copenhagen.

Hab. Cape of Good Hope. Rondebosch (Benson, 1846). Somerset East (Miss Bowker). Stellenbosch (Péringuey). Somerset West (Purcell). .Widely distributed in the Cape Peninsula.

Rhodesia. Bulawayo (in S.A. Museum).

The Rev. E. W. Bowell has identified the animal of specimens from St. James, Cape Peninsula, as typical of the common European cellaria.

183. Polita draparnaudi (Beck).

[S.A.M.]

1801 Helix lucida, Drap., Tabl. Moll. Fr. p. 96. D.

1805 " nitida " Hist. Moll. Fr. p. 117.pl. 8, f. 23-25. D.F. 1815 " lucida " Brard, Hist.Coq.Paris,p.34.pl.2,f.3,4. D.F. 1828 " nitida " Stark, Elem. Nat. Hist. ii. p. 59. D.

1835 " cellaria, Müll., var., Rossmässler, Icon. i. p. 71. pl. 1, f. $22 *$. D.F.

1837 ", (Helicella) draparnaldi, Beck (=nitida, Drap.), Beck, Index Moll. p. 6.

1853 ", draparnaldi, Beck, Pfr., Mon. Hel. iii. p. 86. D.

1854 " " " " Conch. Cab. p. 497. pl. 161, f. 23-25. D.F.

1855 Zonites lucidus, Drap., Moq.-Tand., Hist. Nat. Moll. Fr. ii. p. 75. pl. 8, f. 29-35. D.F.A.

1869 " cellarius, "Müll.," Jeffr., Brit. Conch.v. pl. 9, f. 1. F. 1886 Hyalinia (Polita) lucida, Drap., Tryon, Man. of Conch. ii. p. 149. pl. 48 , f. $37-43,46-56$. pl. 49, f. 57-59. D.F.

1895 Helix nitida, Drap., Locard,Ipsa DraparnaudiConchylia,p.145. N. 1907 Hyalinic (Euhyalinia) lucida, Drap., Tayl., Mon. Brit. Moll. p. 18. pl. 2 (1908), pl. 6 (1909). D.F.A.R.

1911 ", (Polita) lucida, Drap., Germain, Arch. Zool. Exper. vi. p. 234. pl. 13, f. 1-6, 10-12, 22-24, 32-34, 41-43. N.F.

Originals of Zucida, Drap., in Naturh. Hofmus. Vienna (as nitida). Hab. Cape Peninsula. Rondebosch (Oakley). Kenilworth (Connolly).

Specimens from Kenilworth have been dissected and identified by the Rev. E. W. Bowell.

I fail to see how the name lucida, of Draparnaud, can possibly be retained for this species. The earliest Helix lucida is that of Pulteney, 1799 (Cat. Dorset, p. 47), who wrote:- 
"H. licida, M.P.; pellucida, Pen., 134; Gualt.t. 2, G. Testa pellucida, umbilicata, depressa, lævissima.

"Pellucid snail shell, about $\frac{3}{8}$ of an inch long, exactly resembling the foregoing" (H. ericettorim, Müll.) "in figure, but quite smooth and glossy, and wholly without striæ, marks, or bands.

"Found on plants in the River Stour."

(Gualtieri's figure, quoted above, appears to represent a roughly striate shell, somewhat high in the spire, with a distinctly expanded peristome, and his letterpress runs, "Cochlea terrestris umbilicata, minor, pellucida, flavescens.")

Draparnaud's description of his Helix lucida (1801) is as follows :-

"H. lucide. H. lucida. Coq. transparente, luisante, corné clair en dessus, blanchâtre en dessous ; ouverture gránde.

" Haut. 5-6 mill. ; larg. 13-16 ; diam. 11-14.

"Helix cellaria, Müll,; Verm. Hist. 230. Gualt. t. 2. f. G.

"H. commune dans les jardins, sous les haies. (5 tours). Animal pâle, blanchâtré, un peu grisâtre en dessus. Tentacules grisâtres. Yeux noirs."

Müller's original description of cellaria is :-

"H. testa umbilicata, depressa, lutescente, nitida, subtus lactea. Apertura larga."

Pulteney's H. lucida is probably synonymous with cellaria, Müll. ; but it is evident that, in using the same name and quoting the same figure as Pulteney, Draparnaud intended to allude to Pulteney's species; while his reference to Müller, whose description of cellaria he practically translates, shows that in his opinion lucida and cellaria were identical. The fact that in 1805 he rechristened his own $H$. lucida, nitida, and gave the name lucida to $H$. nitida, Müll., does not tend to simplify the matter.

It appears from the foregoing that if the name lucida be allorved to stand at all, it should bear Pulteney's name as author; but under the circumstances it seems preferable to consider lucida, Pult., a synonym of cellaria, and to follow the majority of Continental authorities in adopting a modification of Beck's name, drapamaldi, for the present much-debated species.

Among its other synonyms are nitens, von Alten, 1812; obscurata, Porro in Villa, 1841; blauneri, Shuttl., 1843; fulgida, Parreyss, 1851; planulata, Stabile, 1864; septentrionalis, farinesianus, and navarricus, Bgt., 1870; calabricus, Paulucci, 1879 ; gyrocurta, Bgt. in Servain, 1880; porroi, Paulucci, 1882; subfarinesiana, Bgt.; barbozana, Castro ; and intermissa, Locard, 1894. 
I have known South African examples display cannibal propensities, devouring other small species, even of Zonitoides and Polita, which were placed in their box. They were, however, no match for Natalina coenotera, which treated them in similar fashion.

Sub-FAmily ARIOPHANTINAE, Godwin-Austen, 1888. (Moll. of India, pt. 6, p. 253, as Arionphantinæ, emend. Pilsbry, 1898, Nautilus, xi. p. 129.)

Genus ZONITOIDES, Lehmann, 1862.

(Mal. Blätt. ix. p. 111.)

Type of Genus, Z. nitidus (Müll.).

184. Zonitoides africanus, Böttger.

1910 Zonitoides africanus, Bttg., Abh. Senckenb. Naturf. Ges. Frankfurt, xxxii. p. 436. pl. 28, f. 2. D.F.

Type in Senckenberg Museum, Frankfurt.

Hab. Damaraland, Gobabis (subfossil, Hermann).

\section{Zonitoides ARBoreus (Say).}

1817 Helix arboreus, Say, Nicholson's Encyclopædia, iv. pl.4, f. 4. D.F. 1821 ", arborea , Fér., Tabl. Syst. Moll. pt. 3. p. 45 (or 41). N. 1840 ,, ottonis, Pfr., Archiv. f. Naturg., vi, 1. p. 251. D.

1841 ", arborea, Say, Binn., Boston Journ. Nat. Hist. iii. p. 422. pl. 22 , f. 1. D.F.

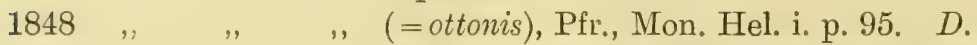

1852 , , , , Pfr., Conch. Cab. p. 114. pl. 85 (1850), f. $33-$ 35. D.F.

1866 Hyalina breweri, Newcomb, Tryon, Amer. Journ. of Conch. ii. p. 250. pl. 19, f. 27. D.F.

1885 Zonites arboreus, Say, Binn., Bull. U.S. Nat. Mus. xxviii. p. 61. $D . F$. and Synonymy.

1886 Hyalinia (Polita) arborea, Say (=breweri, Newc.), Tryon, Man. of Conch. ii. p. 161. pl. 51, f. 9-17. D.F.

Type-ubi?

Ha3. Cape of Good Hope. Port Elizabeth (Layard). Grahamstown (Reeve). Kenilworth (Connolly). King Williamstown (Godfrey). Queenstown (Becker).

Transvaal. Pretoria (Connolly).

Natal. Pietermaritzburg (Ponsonby). 
A common American species, introduced into S. Africa and various other parts of the globe.

186. Zonitoides cupido, Melv. \& Pons.

[S.A.M.]

1903 Zonitoides cupido, M.\& P., A.M.N.H. xii. p. 601. pl. 32, f. 1. D.F. Type in British Museum.

Hab. Zululand. Lower Umfolosi Drift (Burnup).

RHodesia. Victoria Falls (Warren).

\section{Sub-Family ?}

("Position most doubtful ;" Godwin-Austen, 1908, Fauna of Brit. India, Moll., p. 258.)

Genus KALIELLA, Blanford, 1863.

(A.M.N.H. xi.p. 83.)

Type of Genus, $K$. barrakporensis (Pfr.).

187. Kaliella euconuloides, Melv. \& Pons. [S.A.M.]
[S.

1908 Kaliella euconuloides, M.\& P., A.M.N.H.i.p. 133. pl. 7,f.5. D.F. Type in British Museum.

Hab. NataL. Dargle; Karkloof; Inhluzani; Game Pass (Burnup).

The generic position of this species is doubtful ; Colonel GodwinAusten informs me that it is not a Kaliella.

188. Kaliella sigurensis, Godwin-Austen. [S.A.M.]
[. 1882 Kaliella sigurensis, G.-Aust., L. \& F. W. Moll. India, i. p. 5. pl. 1, f. 11. D.F.

1890 Helix (Trochonanina) prètoriensis, M.\&P.,A. M.N.H.vi.p.469: D. 1892

f. 5 . $F$. ix.p.94.pl.4,

Types in British Museum.

Hab. Transvaal. Pretoria District (pretoriensis, Farquhar).

Natal. Pietermaritzburg; Dargle; Equeefa; Karkloof; Tyeloti, near Botha's Hill (Burnup).

Described from the Nilgherri Hills, India.

Colonel Godwin-Austen has identified the Pretorian form of Kaliella as sigurensis, and in all probability the Natal localities refer to the same species. E. A. Smith, however (P.Z.S. 1899, p. 582), in chronicling $K$. barrakporensis (Pfr.), from Mount Chirad- 
zulu, B.C.A., considered that pretoriensis, M. \& P., was a synonym of the last named. It is very possible that both these nearly allied Indian species have found their way into South Africa, and may have been mistaken one for another.

\section{Kaliella victoria, Preston.}

1912 Kaliclla victoria, Prest., A.M.N.H. ix. pp. 69, f. 2; 70. F.D. Type in coll. Preston.

Hab. Rhodesia. Victoria Falls (Connolly).

Familx LIMACID $\mathrm{E}$, Gray, 1821.

(Lond. Med. Repos. xv. p. 230, as Limacider, emend. 1824, Ann. of Philos. viii. p. 107.)

Sub-FAmil LIMACIN E, Swainson, 1840.

(Treatise on Malacology, p. 327.)

Genus LIMAX, Linné, 1758.

(Syst. Nat., Ed. 10. i. pp. 644, 652.)

Type of Genus, L. maximus, Lin.

190. Limax flavus, Linné.

[S.A.M.]

1758 Limax flavus, Lin., Syst. Nat., Ed. 10. i. p. 652. . D.

$1774 \quad$ " , Müll., Verm. ii. p. 10. D.

1801 " variegatus, Drap., Tabl. Moll. Fr. p. 103. D.

1815 Limacella unguiculus, Brard, Hist. Coq. Paris, p. 115. pl. 4, f. $3,4,11,12$. D.F.

1819 Limax variegatus, Drap., Fér., Hist. Nat. Moll. ii, 1. p. 71. pl. 5 , f. 1-6. D.F.

1831 Limacellus unguiculus, Brard (=carinatus, Leach), Turton, Manual, p. 25. $D$.

1840 Limax flavus, Lin., Gray, Turton's Manual,p.114.pl.3, f.16. D.F. 1855 " variegatus, Drap., Moq.-Tand., Hist. Nat. 'Moll. Fr. ii. p. 25. pl. 3, f. 3-9. D.F.A. 1860 " , , , Heynem., Mal. Blätt. vii. p. 165. pl. 1, f. 1-8. D.F.

1863 " . , " Heynem., Mal. Blät. x. p. 212. pl. 3, f. 5. N.R.

$1869 \quad$ " " $\quad$ " Lehm., Mal. Blätt. xvi. p. 148: N. 1885 " flavus, Lin., Tryon, Man. of Conch. i. p. 200. pl. 49, f. $63,70-72$. pl. 50, f. $76 . D . F$. 
1900 Limax variegatus, Drap., Cllge., Ann. S.A. Mus. ii.p. 2. L. 1903 " flavus, Lin., Tayl., Mon. Brit. Moll. p. 78. pl. 10 (1905), f. 3-9. D.F.A.R.

1910 " " , Cllge., Ann. Natal. Mus. ii. p. 160. N.

Hab. Natal. Pietermaritzburg (Burnup).

Cape Peninsula. Cape Town (Lightfoot).

A well-known and widely distributed species, in whose synonymy have been placed L. umbrosus, Phil., 1844; maculatus, Kalenicz, 1851; deshayesii, Bgt., 1862; companyoi, Bgt., 1863; breckworthianus, Lehm., 1864; bicolor, Selenka, 1865; baticus, Mabille, 1868; and ecarinatus, Bttg., 1881.

\section{Limax maxinues, Linné.}

1678 Limax cincreus, Lister, Hist. Anim. Angl. p. 127. pl. 2, f. 15.

D.F. (Pre-Limnean.)

maximus, Lin., Syst. Nat., Ed. 10. i. p. 652. D.

1805 " cinereus, Müll., Verm. ii. p. 5. D.

1815 Limacclla " " Drap.,Hist.Moll.Fr.p.124.pl.9,f.11. D.F.
9, $10 . \quad D . F$.

1819 Limax antiquorum, Fér., Hist. Nat. Moll. ii, 1. p. 68. pl. 4. D.F.

1821 " cinereus, Müll., C. Pfr., Syst. An. u. Beschr. deutsch.

L.-u. W. Schn. p. 20. D.

" $\quad$ " alpinus, Fér., Tabl. Syst. Moll. pt. 2. p. 21. D.

" " valentianus, Fér., Tabl. Syst. Moll. pt. 2. p. 21. D.

1831 Limacellus parma, Brard, Turton, Manual, p. 24. D.

1840 Limax. maximus, Lin., Gray, Turton's Man. p. 112.pl.3, f.14. D.F.

1855 " (Eulimax) maximus, Lin., Moq.-Tand., Hist. Nat. Moll.

Fr. ii. p. 28. pl. 4, f. 1-8. D.F.

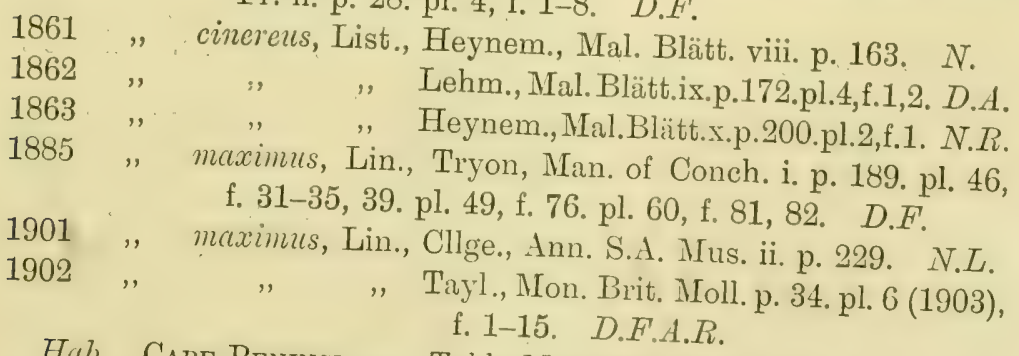

Hab. Cape Peninsula. Table Mountain, Nerwlands (Purcell).

Another European slug, whose recent synonymy includes $L$. cyrenceus, companyoi, and maculatus, Nunnely; sylvaticus, Morelet;
and cornalice, Pini. 
Genus MILAX, Gray, 1855.

(Cat. Pulm. p. 174.)

(=Amalia, Moquin-Tandon, 1855.)

Type of Genus, $M$. gagates (Drap.).

192. Mrlax capensis (Krauss).

1848 Limax capensis, Krs., Südafr. Moll. p. 73. D.

1855 ", kraussii, H. \& A. Ad. (=campestris, “Krs."), H. \& A. Ad., Gen. rec. Moll. ii. p. 219.

$1862 \quad$ " $\quad$, $\quad$ Heynem., Mal. Blätt.ix.p.217. $N$.

, Amalia capensis, Krs., Heynem., Mal. Blätt. ix. p. 215. N.

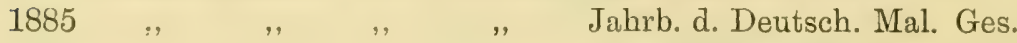
xii. p. $294 . N$.

"Limax , , (=kraussii, Ads.), Tryon, Man. of Conch. i. p. $214 . N$.

1891 Amalia , $\quad$ " Ckll., A.M.N.H. vii. p. 336. N.

Type in Stuttgart Museum.

Hab. Cape of Good Hope (Krauss).

193. Milax gagates (Draparnaud).

1801 Limax gagates, Drap., Tabl. Moll. Fr. p. 100. D.

$1805 \quad$ " " " Hist. Moll. Fr. p. 122. pl. 9, f. 1, 2. D.F. 1819 " " $\quad$ " Fér., Hist. Nat. Moll. ii, 1. p. 75. pl. 6, f. 1, 2. D.F.

1824 " manrus, Quoy \& Gaim., Voy. Uranie, p. 427. D.

1855 Milax gagates, Drap., Gray, Cat. Pulm. p. 174. D.

" Limax (Amalia) gagates, Drap., Moq.-Tand., Hist. Nat. Moll. Fr. ii. p. 19. pl. 2, f. 1-3. D.F.

1870 " $\quad$ " $\quad$, Drap., Semp., Reis. in. Arch. Philippin. ii, 3. p. 84. pl.11, f. 9, 26. A.R.

1885 Amalia gagates, Drap., Heynem., Jahrb. d. Deutsch. Mal. Ges. xii. p. $293 . \quad N$.

Tryon, Man. of Conch. i. p. 218. pl. 53, f. 53,54 . D.F.

$1891 \quad$, $\quad$,, $\quad$, Ckll., A.M.N.H. vii. pp. 329, 337. N.

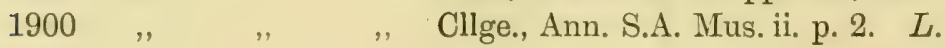

$1901 \quad$ " " " " " $\quad$ p. $230 . \quad$.

1904 " , " $\quad$ Tayl., Mon. Brit. Moll. p. 139. pl. 15 (1906), f. 9-14. D.F.A.R.

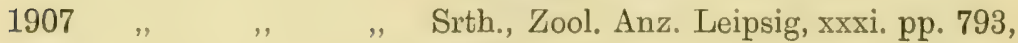
798. $N$.

1910 Milax gagates, Drap., Cllge., Ann. Natal Mus. ii. p. 161. N. 
Ital. Cape of Good Hope. Port Elizabeth (fide Cockerell). Cape Town (Lightfoot). Cape Flats (Schultze). Ashton; Storms Vlei (Purcell). Simonstown (German South Pole Expedition).

NataL. Pietermaritzburg (Burnup).

A European species, known from many parts of the globe. Other synonyms are hewstoni, Cooper, 1872; marginata, Pini, 1876; tasmanicus and ?nigricolus, Tate, 1880; and babori, Cllge., 1897.

194 Milax ponsonbxi (Collinge).

1900 Amalia ponsonbyi, Cllge., Ann. S.A. Mus. ii. p. 2. pl. 1, f. 1, 2.

1910 pl. 2, f. 13. D.F.A.

") (?=capensis, Krs. $)$, Srth., Deutsch. Sudpol. Exp. xii. p. 157. N.

Type in University Museum of Zoology, Cambridge.

Hab. Cape Peninsula. Cape Town (Lightfoot).

Genus AGRiolimax, Mörch, 1865.

(J. de C. xiii. p. 378.)

Type of Genus, $A$. agrestis (Lin.).

195. Agriolinax agrestis (Linné).

1758 Limax agrestis, Lin., Syst. Nat., Ed. 10. i. p. 652. D.

$1774 \quad$ " " Müll., Verm. ii. p. 8. D.

" " $\quad$ reticulatus, Müll., Verm. ii. p. 10. D.

1791 " filans, Hoy, Linn. Trans. i. p. 183. D.N.

1805 ", agrestis, Lin., Drap., Hist. Moll. Fr.p.126.pl.9, f. 9. D.F. 1815 Limacella obliqua, Brard, Hist. Coq. Paris, p. 118. pl. 4, f. 5, 6, 13-15. D.F.

1819 Limax bilobatus, Fér., Hist. Nat. Moll. ii, 1. p. 74. pl. 5, f.2. D.F. " " agrestis, Lin., Fér., ibid. p. 73.pl. 5, f. 7-10. D.F.

1831 Limacellus obliquus, Brard, Turton, Manual, p. 26. D.

1840 Limax agrestis, Lin., Gray, Turton's Man. p. 117.pl. 3,f.17. D.F'

1855

" " " Moq.-Tand., Hist. Nat. Moll. Fr. ii. p. 22. pl. 2, f. 18-22, pl. 3, f. 1, 2. D.F.

$1862 \quad$ " , " Lehm., Mal. Blätt. ix. p. 183. D.

$1863 \quad$ " " " Heynem., Mal.Blätt.x.p.209.pl.2,f.7. N.R

1885 " (Agriolimax) agrestis, Lin., Tryon, Man. of Conch.

p. 205. pl. 50, f. 90-94. pl. 51, f. 95-98. D.F.

1893 Agriolimax agrestis, Lin., Ckll., Conchologist, ii. p. 200. N. 1900 " Cllge., Ann. S.A. Mus. ii. p. 3. L. 
1903, 4 Agriolimax agrestis, Lin., Tayl., Mon. Brit. Moll. p. 104. pl. 15 (1906), f. 1-4. D.F.A.R.

1907 Srth., Zool. Anz. Leipsig, xxxi. pp. 793, 798. $N$.

1910 Cllge., Ann. Natal Mus.ii. p. 161. N.

Hab. Cape Peninsula. Cape Town (Lightfoot). Cape Flats (Schultze). Green Point (Roebuck). Miller's Point (German South Pole Expedition).

Natat. Pietermaritzburg (Burnup).

A species of world-wide distribution, which has been described under many names.

In addition to the early synonymy given above, the following are, by various authorities, considered to be identical with agrestis: Limax salicium, Bouillet; tunicata, Gould; pallidus, Schrenk; niciensis and veranyanus, Bgt.; heydeni, Heynem.; norvegicus, Westerl. ; fedtschenkoi, Koch \& Heynem.; panormitanis, Less. \& Poll.; mentonicus, Nev.; varians, A. Adams; molestus, Hutton; and dymczeviczii, Kalenicz.

\section{Agriolmiax Levis (Müller).}

1774 Limax lavis, Müll., Verm. ii. p. 1. D.

1801 " brunneus, Drap., Tabl.-Moll. Fr. p. 104. D.

1821 " lavis, Müll., and brunneus, Drap., Fér., Tabl. Syst. Moll. pt. 2. p. $23 . \quad D$.

1885 Agriotimax lavis, Müll., Srth., Zeitschr. f. wiss. Zool. Leipsig, xlii. p. 327. pl. 7, f. 17. D.F.

"Limax (Krynickia) lavis, Müll., Tryon, Man: of Conch. i. p. 211. pl. 52, f. 21. D.F.

1898 Agriolimax lavis, Müll., Stur., S.A. Moll. p. 36. N. 1904 " (Hydrolimax) lavis, Müll., Tayl., Mon. Brit. Moll. p. 121. pl. 15 (1906), f. 5-8. D.F.A.R.

Hab. Cape of Good Hope. Queenstown (Dower; Roebuck).

Other synonyms of this world-diffused slug appear to be: lacustris, Bonelli, 1822 ; campestris, Binn., 1841 ; lombricoides, Morel., 1845 ; parvulus, Normand, 1852 ; arenarius, Gass., 1867; rarotonganas, Heynem., 1871 ; argentinus, Strob., 1874; montanus and castaneus, Ing., and ingersolli, Binn., 1875; hyperboreus, Westerl., 1876; meridionalis, Doering, 1878; stenurus, Strebel, 1880 ; brasiliensis, Semper, 1885 ; queenslandicus, Hedley, 1888; and bevenoti, Cllge., 1897. 
FAMILY UROCYCLIDÆ, Simroth, 1889.

(Nov. Act. Acad. Caes. Leop. liv. p. 62.)

\section{Genus UROCYCLUS, Gray, 1864.}

(P.Z.S. p. 250.)

Type of Genus, U. kirki, Gray.

197. Urocyclus fasciatus, von Martens.

1884 Urocychus fasciatus, von Mits., Heynem., Jahrb. d. Deutsch. Mal. Ges. xi. p. 8. pl. 1, f. 4, 5. D.F. $1910 \quad$ " $\quad$ vonMts.,Cllge.,Ann.NatalMus.ii.p.162. N.

Type in Zool. Mus. Berlin.

Hab. Mozambique. Quilimane (fide Simroth).

Natal. Pietermaritzburg (Burnup).

\section{Urocyclus fLAVescens (Keferstein).}

1866 Parmarion flavescens, Kfstn., Mal. Blätt. xiii. p. 70. pl. 2, f. 1-8. D.F.A.R.

1879 Urocyclus flavescens, Kfstn. (cum var. pallida), Gibbons, Journ. of Conch. ii. p. 138. D.

\begin{tabular}{|c|c|c|c|}
\hline 1883 & ", & ," & $\begin{array}{l}\text { Pfeffer,Abhandl.Naturwiss. Verein. } \\
\text { Hamburg, vii, 2. p. } 12 . \text { D. }\end{array}$ \\
\hline 1884 & ", & $"$ & $\begin{array}{l}\text { Heynem., Jahrb. d. Deutsch. Mal. } \\
\text { Ges. xi. p. 6. pl. } 1 \text {, f. } 2,3 . \text { D.F. }\end{array}$ \\
\hline 1885 & ", & ," & $\begin{array}{l}\text { (cum var. pallida, Gibb.), Tryon, } \\
\text { Man. of Conch. i. p. 163. pl. } 35 \text {, } \\
\text { f. } 34-36 \text {. D.F. }\end{array}$ \\
\hline & & , & Ckll., Journ. of Mal. iii. p. 52. D. \\
\hline & ", & " & Cllge., Ann. Natal Mus.ii.p.162. \\
\hline
\end{tabular}

Type in Zool. Mus. Berlin.

Hab. Natal. Pinetown. Pietermaritzburg (Burnup).

Lorenzo Marques. Inhambane; Quilimane; Mungurumbe (Peters, 1846). Delagoa Bay (Spencer).

199. Urocyclus hinki, Gray.

1864 Urocychus kirkii, Gray, P.Z.S. p. 251. D.F.

1879

1884

19
" Gibbons, Journ. of Conch. ii. p. 139. N.

", Tryon, Struct. \& Syst. Conch. iii. p. 81. pl. 101, f. 60. D.F.

"Heynem., Jahrb. d. Deutsch. Mal. Ges. xi. p. 7. pl. 1, f, 1. D.F. 
1864 Urocychus kirkii, Gray, Binn., Ann. N.Y. Acad. Sci. iii. p. 84. pl. 16 , f. K. pl. 17 , f. N. $R$.

1887 " Poirier, Bull. Soc. Mal. Fr.iv.p.196.pl.5, f. 1, 2,6.pl.6, f. 1, 2.pl.s, f.1. D.F.A.R.

1891 " $\quad$ " Clill, A.M.N.H. vii. p. 101. N.

$1910 \quad$ ", Cllge., Ann. Natal Mus. ii. p. 161. N.

Type in British Museum.

Hab. Natal. Durban (fide Sturany).

Mozambique. Near the mouth of the Zambesi (Kirk).

Lorenzo Marques. Delagoa Bay; Inhambane (fide Sturany).

200. Urocyclus kraussianus (Heynemann).

1848 Limax (Arion) ? sp., Krs., Südafr. Moll. p. 73. D.

1862 " kraussianus, Heynem., Mal. Blätt. ix. p. 217. D.

$1863 \quad$ " " $\quad$ " $\quad$ ".p.211.pl.3,f.2.N.R.

1891 Urocyclus ", $\quad$ " Ckll., A.M.N.H. vii. p. 102. N.

$1910 \quad$ " , , Clige.,Ann.NatalMus.ii.p.163.N.

Hab. Cape Peninsula. Cape Town (Krauss).

Natal (fide Sturany).

201. Urocyclus pallescens, Cockerell.

1891 Urocychus pallescens, Ckll, A.M.N.H. vii. p. 101. D.

$1910 \quad$ " " Cllge., Ann. Natal Mus. ii. p. 162. D.

Type in British Museum.

Hab. Natal. Durban (Craven).

FAmily ARIONID无, Gray, 1840.

('Turton's Manual, pp. 101, 104.)

Sub-FAmily OOPELTIN AE, Cockerell, 1891.

(1.Z.S. p. 216.)

Genus OOPELTA, Mörch, 1867.

(Mal. Blätt. xiv. p. 191.)

Type of Genus, O. nigropunctata, Mörch.

202. Oopelta atemrina (Gray).

Limax (Arion) allerian, Gray, in British Museum.

1855 Arion aterrimus, Gray, Cat. Pulm. p. 55. D.

1890

(? Oopelta),Ckll.,A.M.N.H.vi.p.387. D.N,

1901 Oopelta aterrima " Cllge., Ann, S.A. Mus. ii. p. 230. D. 
Type in British Museum.

Hab. South Africa (fide Gray).

203. Oopelta capensis, Pollonera.

1909 Oopelta capensis, Pollon., Boll. Mus. Torino, xxiv, 608. p. 11 plate, f. 1-6. D.F.

Type in Museum of Zoology, Turin.

Hab. Cape of Good Hope (in Mus. Torino).

204. Oopelta flavescens, Collinge.

1900 Oopelta flavescens, Cllge., Ann. S.A. Mus. ii. p. 6. pl. 1, f. 9, 10.

1901

$$
\text { pl. 2, f. 17. D.F.A. }
$$

Type in University Museum of Zoology, Cambridge.

Hab. CAPE of Good Hope. Kalk Bay; Caledon; Swellendam; Kogman's Kloof, Ashton (Purcell).

\section{Oopelta granulosa, Collinge.}

1900 Oopelta granulosa, Cllge., Ann. S.A. Mus. ii. p. 6. pl. 1. f. 11, 12.

1901 pl. 2, f. 18 . D.F.A.

Type in University Museum of Zoology, Cambridge.

Hab. CAPE of Good Hope. Nieuwondtville (Leipoldt). Hot Springs, Montagu (Purcell). Sir Lowry's Pass (Connolly).

\section{Oopelta minor, Pollonera.}

1909 Oopelta minor, Pollon., Boll. Mus. Torino, xxiv, 608. p. 12. plate, f. 7, 8. D.F.

Type in Museum of Zoology, Turin.

Hab. Cape of Good Hope (in Mus. Torino).

207. Oopelta nigropunctata, Mörch.

1867 Oopelta nigropunctata, Mörch, Heynem., Mal. Blätt. xiv. p. 191.

1885

1900

1901

1909 pl. 2, f. 1, 2. D.R.

Tryon, Man. of Conch.i.p.225. D.

Cllge., Ann. S.A.Mus.ii.p. 5. pl.1, f. 7, 8. pl. 2, f. 16 . D.F.A.

Cllge., Ann. S.A. Mus. ii. p. 231. pl. 14, f. 6, 11, 12 . A.

Pollon., Boll. Mus. Torino, xxiv, 608. p. 10. D, 
1910 Oopelta nigropunctata, Mörch, Srth., Deutsch. Sudpol.Exp.xii,4. p. 149. pl. 21, f. 1-14, 16, 17. $A$.

Hab. Cape of Good Hope. Cape Town (Lightfoot). Bergvliet (Schultze). Albany District (Penther). Sir Lowry's Pass (Connolly).

Described from Guinea.

208. Oopelta polypunctata, Collinge.

1901 Oopelta polypunctata, Cllge., Ann. S.A. Mus. ii. p. 232. pl. 14, f. $3,4,7,8,9,10$. D.F.A.

Type in University Museum of Zoology, Cambridge.

Hab. Cape of Good Hope. Zonder End, Caledon Division (Purcell).

Sub-Family ARIONIN 2 , Morse, 1864.

(Journ. Portland Soc. Nat. Hist. i, 1. pp. 5, 7.)

Genus ARION, Férussac, 1819.

(Hist. Nat. Moll. ii, 1.-pp. 50, 53.)

Type of Genus, A. empiricorum, Fér.

209. Arion fuscus (Müller).

[S.A.M.]

1774 Limax fuscus, Müll., Verm. ii. p. 11. D.

$1821 \quad$ " $\quad$ Fér., Tabl. Syst. Moll. pt. 2. p. 23. D.

1855 Arion fuscus, Müll., Moq.-Tand., Hist. Nat. Moll. Fr. ii. p. 14. pl. 1, f. 28-30. D.F.

1863 " , Mörch, Vid. Med. naturh. For. Copenhagen, p. $273 . \quad D$.

1873 ", Lehm., Leb. Schneck. Stettins, p. 17.pl. 2, f. 2. D.F.

1885 (=subfuscus, Drap., cinctus, Dumont, fasciatus, Nilss. (part), and incommodus, Hutt.), Tryon, Man. of Conch. i. p. 235. pl. 57, f. 18-21. D.F.

$1887 \quad$ " $\quad$, Pollon., Atti. Acc. Sci. Torino, xxii. p. 200. pl. 3, f. 14. D.F.

$1890 \quad$ " $\quad$ " Pollon., Boll. Mus. Zool.Torino, v.p. 12. D.

$1893 \quad$ " " Ckll., Conchologist, ii. p. 211. N.

$1897 \quad " \quad$ " (=citrinus, Westerl., 1871, and stabilis, Pollon., 1885), Cllge., P. Z.S. p. 443. pl, 30, f, 13. pl. 31, f, 14-16, D.A. 
1900 Arion fuscus, Müll., Cllge., Ann. S.A. Mus. ii. p. 7. L. 1910 Ann. Natal Mus. ii. p. $170 . L$.

Hab. Cape Peninsula. Cape Town (Lightfoot).

Natal. Pietermaritzburg (Burnup).

\section{Arion intermedius, Normand.}

1852 Arion intermedins, Norm., Descr. Lim. nouv. p. $6 . \quad D$. 1867 Geomalacus intermedius, Norm., Mab., Rev. et Mag. Zool.p.57. D. 1887 Arion intermedius, Norm., Pollon., Atti. Acc. Sci. Torino, xxii.

\begin{tabular}{|c|c|c|c|}
\hline 189 & & & p. 207. pl. 3, f. 1-7. D.F. \\
\hline & , & , & Ckll., ibid. pp. 63, 212. D.A.N. \\
\hline 906 & , & ", & $\begin{array}{l}\text { Tayl., Mon. Brit. Moll. p. } 240 . \text { pl. } 24 \\
\text { (1907), f. 18-23. D.F.A.R. }\end{array}$ \\
\hline 07 & " & ", & $\begin{array}{l}\text { Srth., Zool. Anz. Leipsig, xxxi. } \\
\text { pp. } 794,796 . \quad \text { N. }\end{array}$ \\
\hline & " & " & $\begin{array}{l}\text { Srth., Deutsch. Sudpol. Exp. xii, } \\
\text { p. } 153 . \quad N .\end{array}$ \\
\hline
\end{tabular}

Hab. Cape Peninsula. Cape Flats (Schultze).

Although first differentiated at so comparatively recent date, this European species has the following synonyms: Geomalacus hiemalis, Drouet, 1867; mabillei, Baud, 1868 ; vendeanus, Let., 1869 ; Arion verrucosus, Brev., 1881; mabillianus, Baud, 1884 ; minimus, Srth., 1885 ; and mollerii, Pollon., 1889.

FAMILY ENDODONTID E, Crosse, 1894.

(J. de C. xlii. p. 219.)

Grnus ENDODONTA, Albers, 1860.

(Die Helic. p. 90.)

Type of Genus, E. lamellosa (Fér.).

Section AFRODONTA, Melv. \& Pons, 1908.

(A.M.N.H. i. pp. 133, 135.)

Type of Section, E. bilamellaris, M. \& P.

211. Endodonta (Afrodonta) bilamellaris, Melv. \& Pons.

1908 Afrodonta bilamellaris, M.\& P., A.M.N.H.i.p.134.pl.7,f.6. D.F. 
1912 Endodonta (Afrodonta) bilamellaris, M. \& P., Bnp., Ann. Natal Mus. ii. p. 335. pl. 24, f. 18. D.N.F.

Type in British Museum.

Hab. NataL. Dargle; Edendale; Tongaat; Enon bush, Richmond; Hilton Road; Ntimbankulu (Burnup).

212. Endodonta (Afrodonta) farquhari, Burnup.

1912 Endodonta (Afrodonta) farquliari, Bnp., Ann. Natal Mus. ii. p. 339. pl. 24, f. 7-10. D.F.

Type in British Museum.

Hab. Cape of Good Hope. Grahamstown; Port Elizabeth; Kowie; Bathurst (Farquhar).

213. Endodonta (Afrodonta) inhluzaniensis, Burnup.

1912 Endodonta (Afrodonta) inhluzaniensis, Bnp., Ann. Natal Mus. ii. p. 342 . pl. 24 , f. 14-17. D.F.

Type in British Museum.

Hab. Natal. Inhluzani Mountain (Burnup).

214. Endodonta (Afrodonta) novemlameltaris, Burnup. [S.A.M.]

1912 Endodonta (Afrodonta) novemlamellaris, Bnp., Ann. Natal Mus. ii. p. 341. pl. 24 , f. 11-13. D.F.

Type in British Museum.

Hab. Cape of Good Hope. Grahamstown; Kowie (Farquhar). King Williamstown (Godfrey).

215. Endodonta (Afrodonta) perfida, Burnup.

1907 Trachycystis rotula, M.\&P., A.M.N.H.xix. p.99. pl. 6, f. 12. D.F. 1912 Endodonta (Afrodonta) perfida, Bnp. (=rotula, M. \& P., 1907, nec Hombr. \& Jacq., 1854), Bnp., Ann. Natal Mus. ii. p. 337. pl. 24, f. 1-6. D.F.

Type in British Museum.

Hab. CAPE of Good Hope. Fern Kloof, Grahamstown (Farquhar).

216. Endodonta (Afrodonta) trilameldaris, Melv. \& Pons.

1908 Afrodonta trilamellaris, M.\&P., A.M.N.H.i.p. 134.pl.7, f.7. D.F. 1912 Endodonta (Afrodonta) trilamellaris, M. \& P., Bnp., Ann. Natal Mus. ii. p. 336. D.N. 
Type in British Museum.

Hab. Natal. Dargle (Buinup).

Genus Phortion, Preston, 1910.

(A.M.N.H. vi. p. 531.)

(=Phasis, Albers, 1850, nec Hubner, in Lepidoptera, 1816.)

Type of Genus, Ph. menkeamum (Pfr.).

217. Phortion capense (Pfeiffer).

1841 Helix capensis, Pfr., Symb. i. p. 40. D.

1848

1849

1850

1851

1853
" (=irrorata, Zgl. in litt.), Pfr., Mon. Hel. i. p. $60 . \quad D$.

Conch.Cab.p.220.pl.34(1848), f.9-11. D.F. " Bs., A.M.N.H. v. p. 217. N.

" Rve., Conch. Icon. pl. 43, f. $202 . \quad$ D.F.

$"$ (=littoricola, Bs. in litt.), Pfr., Mon. Hel. iii. p. 66.

1887 Helix (Pella) capensis, Pfr., Tryon, Man. of Conch. iii. p. 103. pl. 20, f. 61. D.F.

Type in Stuttgart Museum.

Hab. Cape of Good Hope (coll. Pfeiffer). Cape Town and Cape Peninsula (very common). Robben I. (Fisk). Dassen I. (Connolly). Port Elizabeth to Alexandria (Crawford). Port Alfred (Penther). Widely distributed from Port Elizabeth to Namaland. Ph. paludicola (Bs.) is possibly a small, and vitenhagense (Krs.) a high-spired, form of capense, while namaquanum (von Mts.) appears to be little more than a variety of the same species, of which examples quite as large as namaquanum are known from Muizenberg.

218. Phontion menteanum (Pfeiffer).

1842 Helix imenteana, Pfr. (H. capensis, Menke, ined.), Pfr., Symb. ii. p. 33. D.

1848 ", Mon. Hel. i. p. $55 . \quad$ D.

" $\quad " \quad \quad$ " Krs., Südafr. Moll. p. 75. N.

$1852 \quad$ ", $\quad$, Conch. Cab. p. 229.pl.133, f. 5, 6.pl. 111 (1850), f. 1-5. D.F.

," Rve., Conch. Icon. pl. 106, f. 591. D.F.

1887 Helix (Pella) menkeena, Pfr., Tryon, Man. of Conch. iii. p. 108. pl. 21, f. 98, D.F.

1893 Phasis menkeana, Pfr., Pilsb., Man. of Conch. ix. p. 37. pl. 10, f. $1-3$. F. 
Type in Stettin Museum.

Hab. Cape of Good Hope (Menke). Elim (Krauss).

Cape Peninsula, widely distributed from Table Mountain to Cape Point.

219. Phortion mamaquanum (von Martens).

1889 Helix (Pella) namaquana, von Mts., Sitz.-Ber. Ges. Nat. Fr. Berlin, p. 161. D.

1893 Phasis namaquana, von Mts., Pilsb., Man. of Conch, viii.p.297. N. 1894 Helix , " , Conch. Mitth. iii, 3. p. 4. D.

1897 ", , , Archiv. f. Naturg. Ixiii, i. p. 38. pl. 7, f. 1-4. D.F.

Type in Zool. Mus. Berlin.

Hab. Little Namialand. Between Port Nolloth and Ananas (Schenck; Day).

220. Phortion paludicola (Benson).

1850 Helix paludicola, Bs., A.M.N.H. vi. p. 253. D. 1853, $54 \quad$ " „ Pfr., Conch. Cab. p. 358. pl.137, f.4,5. D.F. $1853 \quad$ " $\quad$ " " Mon. Hel. iii. p. 66. D.

" " " $\quad$ " Rve., Conch. Icon. pl. 174, f. 1179. D.F. 1887 Helix (Pella.) paludicola, Bs., Tryon, Man. of Conch. iii. p. 104. pl. 20, f. 64. D.F.

Specimens ex auct. in University Museum of Zoology, Cambridge.

Hab. Cape Peninsula, "Baszaarms" (Baas Harman's) Kraal, near Retreat (Benson). Seekoe Vlei (Connolly).

221. ? Phortion soluers (Melv. \& Pons.)

1907 ? Phasis sollers, M.\& P., A.M.N.H.xix. p. 100. pl. 6, f. 14 . D.F. Type in British Museum.

Hab. Zululand. Melmoth (Miss Hickey).

Probably better placed nearer Trachycystis.

\section{Phortion uitenhagense (Krauss).}

1846 Helix uitenhagensis, Krs., Pfr., Symb. iii. p. 66. D. $1848 \quad$ ", " , Mon. Hel. i. p. 61. D.

" $\quad$ " Südafr. Moll. p. 76. pl. 4, f. 22 . D.F. $1854 \quad$ " Rve, Conch.Tcon.pl.207,f.1461. D.F. 1887 Helix (Pella) vitcnhagensis, Krs., Tryon, Man. of Conch. iii. p. 104. pl. 20, f. 63 . D.F.

Type in Stuttgart Museum.

Hab. CAPE OF GOOD Hope. Winterhoek Mt., Uitenhage 
(Krauss). "Moderately plentiful in the neighbourhood of the village of Uitenhage, on the gravelly soil on low bushes" (Layard). Crawford considers this species to be a var. of capense, and writes of its occurrence at Port Elizabeth: "P. vitenhagense is found only in Zwartkops valley, and is always more conical than capense. Same habits as capense."

Layard wrote: "I look upon this as the inland variety of the preceding species" (capense). "It was not nearly so abundant as capensis in its own locality. It has more whorls and a more exserted spire than capensis, but partakes of all the varieties from pure white to dark mottled specimens."

\section{Genus SCULPTARIA, Pfeiffer, 1855 \\ (Mal. Blätt. ii. p. 135. .)}

Type of Genus, S. sculpturata (Gray).

The subjoined classification is based on J.H. Ponsonby's article in Proc. Mal. Soc., 1910, vol. ix. p. 34.

223. Sculptaria coltaris (Pfeiffer).

1867 Helix collaris, Pfr., Mal. Blätt. xiv. p. 197. D.

$1868 \quad$ " " Mon. Hel. v. p. 506. D.

$1869 \quad$ " " Novit. Conch. iii. p. 496. pl. 107, f.5-9. D.F.

1886 Helix sculpturata, Gray, Kob., Conch. Cab. p. 621. pl. 178, f. 26-

28. D.F.

1887 Polygyra (Sculptaria) sculpturata, Gray, var. collaris, Pfr., Tryon, Man. of Conch. iii. p. 138. pl. 24, f. 27, 28. D.F.

1910 Sculptaria collaris, Pfr., Pons., Proc. Mal. Soc. ix. p. 35. N.

" Helix (Sculptaria) collaris, Pfr., Bttg., Abh. Senckenb. Naturf.

Ges. Frankfurt, xxxii. p. 438. N.

Type in Stettin Museum.

Hab. Damaraland (fide Böttger).

224. Sculptaria damarensis (H. Adams).

1870 Helix (Corilla) damarensis, H. Ad., P.Z.S. p. 379. pl. 27, f. 14. D.F. 1887 Polygyra (Sculptaria) sculpturata, Gray, var. damarensis, H. Ad., Tryon, Man. of Conch. iii. p. 138. pl, 25, f. 67, 68. D.F. 1890 Sculptaria chapmanni, Ancey, Bull.Soc. Mal. Fr., vii. p. 156. D. 1892 ", melvilliana, Ancey (=chapmanni, Ancey, nec Cox), Brit. Nat. p. 126.

1910 " damarensis, H. Ad., Pons., Proc. Mal. Soc. ix. p. 35. N. 
1910 Helix (Sculptaria) damarensis, H. Ad., Bttg., Abh. Senckenb. Naturf. Ges. Frankfurt, xxxii. p. 438. N.

Type of damarensis in British Museum; melvilliana in coll. Dautzenberg.

Hab. Ovampoland. Walwich Bay (melvilliana, Andersson and Chapman).

Pfeiffer, Clessin, and von Martens have at different times placed the present species in the synonymy of collaris, Pfr., and Paetel and Tryon in that of sculpturata, Gray. Ponsonby (1910) remarks: "This is the largest species of the Genus; the spire is more raised and the sculpture is weaker than in any of its allies."

\section{Sculptaria retisculpta (von Martens).}

1889 Helix retisculpta, von Mts., Nachrichtsbl. d. Deutsch. Mal. Ges. xxi. p. $154 . D$.

1894 Sculptaria retisculpta, von Mts., Conch. Mitth. iii, 3. p. 5. D. 1897 " Archiv. f. Naturg. lxiii, i. p. 38. pl. 7, f. 5-7. D.F.

$1910 \quad$ " $\quad$ " Pons.,Proc.Mal.Soc.ix.p.36. N.

Type in Zool. Mus. Berlin.

Hab. Damaraland. Ussab (ex "Limnca," fide von Martens).

\section{Sculptaria sculpturata (Gray).}

1838 Helicodonta sculpturata, Gray, Alexander's Expedition,ii.p.268. D. 1845 Helix sculpturata, Gray, Pfr., Zeitschr. f. Malak. p. 86. D. iii. p. 138. pl. 25, f. 69 . D.F.

1893 Sculptaria sculpturata, Gray, Pilsb., Man. of Conch. ix. p. 39. pl. 10, f. 4. F.

$1910 \quad$, ， " Pons., Proc. Mal. Soc.ix.p.34. D. " Helix (Sculptaria) sculpturata, Pfr., Bttg., Abh. Senckenb. Naturf. Ges. Frankfurt, xxxii. p. 437. N.

Type in British Museum.

Hab. Great Namaland. Near the Great Fish River (Alexander). Damaraland. "Kurikaubmund am Swakop (Kurikop bei Otjikango)" (Rintelen).

var. Rinteleni, Böttger.

1910 Helix (sculptaria) sculpturata, Pfr., var. rinteleni, Bttg., Abb. Senckenb. Naturf. Ges. Frankfurt, xxxii.p.437.pl.28,f.1. D.F. 
Type in Senckenberg Museum, Frankfurt.

Hab. Damaraland. Huleb, S. of Usakos (Rintelen).

Genus TRACHYCYSTIS, Pilsbry, 1893.

(Man. of Conch. viii. p. 136, and ix. p. 37.)

( = Pella, Albers, 1860 (pars), nec Stephens, in Coleoptera, 1835.)

Type of Genus, T. bisculpta (Bs.).

During recent years this Genus has been a convenient receptacle for a large number of small South African helicoids, whose soft parts are mostly unknown, and whose conchological characters do not admit of their inclusion in other South African Genera. It has long been obvious that, as more becomes known of the animals, the Genus will have to undergo considerable revision. The fact, too, of bisculpta standing as the type will enormously restrict Trachycystis s.s., as its animal is a very peculiar one, to which only charybdis, and probably tollini, vorticialis, and hartvigiana are near akin.

227. Trachycystis actinotricha (Melv. \& Pons.). [S.A.M.] 1892 Helix (Pella) actinotricha, M. \& P., A.M.N.H. x. p. 238. pl. 13, f. $5 . \quad D . F$.

1893 Phasis (Trachycystis) actinotricha, M. \& P., Pilsb., Man. of Conch. viii. p. 143. pl. 35, f. 14 . D.F.

Type in British Museum.

Hab. Natal. Pietermaritzburg; Karkloof; Nottingham Road (Burnup).

\section{Trachycystis enea (Krauss).}

1848 Helix cenea, Krs., Südafr. Moll. p. 75. pl. 4, f. 18. D.F.

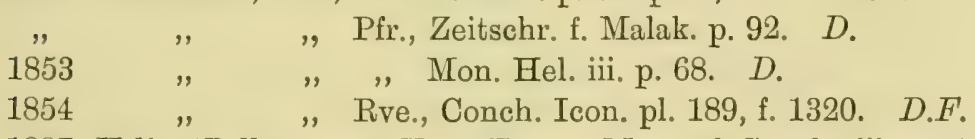

1887 Helix (Pella) anea, Krs., Tryon, Man. of Conch. iii. p. 105. pl. 20, f. 71. D.F.

Type in Stuttgart Museum.

Hab. NataL (Wahlberg).

229. Trachycystis alcocki, Melv. \& Pons.

1895 Helix (Trachycystis) alcocki, M. \& P., A.M.N.H. xv. p. 164. pl. 12, f. 2. D.F. 
Type in British Nuseum.

Hab. Cape of Good Hope. Kowie, Port Alfred to Alexandria District (Crawford). Grahamstown (Farquhar).

230. Trachycystis aprica (Krauss).

1848 Helix aprica, Krs., Südafr. Moll. p. 77. pl. 4, f. 26. D.F.

$\begin{array}{llll}\prime \prime & " & , & \text { Pfr., Zeitschr. f. Malak. p. 114. D. } \\ 1851 & " & , & \text { Rve., Conch. Icon. pl. 43, f. 201. D.F. } \\ 1853 & \quad, & \text { " } & \text { Pfr., Mon. Hel. iii. p. 123. D. }\end{array}$

1887 Helix (Pella) aprica, Krs., Tryon, Man. of Conch. iii. p. 107. pl. 20, f. 88. D.F.

Type in Stutgart Museum.

Hab. Natal (Wahlberg).

231. Trachycystis aulacophora (Ancey).

1890 Helix aulacophora, Ancey, Bull. Soc. Mal. France, vii. p. 153. D. 1893 Phasis (Trachycystis) aulacophora, Ancey, Pilsb., Man. of Conch, viii. p. 138. pl. 43, f. 60, 61. D.F.

Type in coll. Geret.

Hab. Cape of Good Hope. Port Elizabeth, North end to Zwartkops River (Crawford).

232. Trachycystis Bathycoele (Melv. \& Pons.). [S.A.M.]

1892 Helix (Pella) bathycoele, M. \& P., A.M.N.H.ix.p.89.pl.5, f.4. D.F. 1893 Phasis (Trachycystis) bathycoele, M. \& P., Pilsb., Man. of Conch. viii. p. 139, pl. 35, f. 13 . D.F.

Type in British Museum.

Hab. Cape of Good Hope. Craigie Burn, Somerset East (Miss Bowker). Bedford; Dassie Krantz, Grahamstown (Farquhar). Van Staadens River, Port Elizabeth (Crawford).

Natal. Pietermaritzburg; Ntimbankulu (Burnup).

233. Trachycystis bisculpta (Benson).

[S.A.M.]

1851 Helix bisculpta, Bs., A.M.N.H. vii. p. 103. D.

$1853 \quad$ ", " Pfr., Mon. Hel. iii. p. 72. D.

" $\quad$ " $\quad$ Rve., Conch. Icon. pl. 173, f.1171. D.F.

1854 " „ Pfr., Conch. Cab. p. 380. pl. 141 (1852), f. 17-19. D.F.

1887 Helix (Pella) bisculpta, Bs., Tryon, Man. of Conch. iii. p. 105. pl. 20, f. 72. D.F. 
1893 Phasis (Trachycystis) bisculpta, Bs., Pilsb., Man. of Conch. ix. pp. 37, 38. pl. 10, f. 5-7. pl. 15, f. 3, 4. F.A.

Specimens ex auct. in University Museum of Zoology, Cambridge. Hab. Cape of Good Hope. Camps Bay (Benson). Houw Hoek (Lightfoot). Widely distributed over the greater part of the Cape Peninsula, from Lion's Head to Cape Point.

Layard wrote: "There are two forms of this shell; var. major is found at Bredasdorp ; the var. minor I procured among grass roots at the foot of the Round Battery, Simonstown."

A beautiful variety with white, translucent shell, from Signal Hill, Cape Town (Lightfoot), is in the S.A. Museum.

234. Trachycystis burnupi, Melv. \& Pons.

1892 Helix (Pella) bumupi, M.\&P., A.M.N.H.x. p. 239.pl.13, f. 6. D.F. 1893 Phasis (Trachycystis) burnupi, M. \& P., Pilsb., Man. of Conch. viii. p. 140. pl. 35 , f. 5 . D.F.

Type in British Museum.

Hab. NataL. Pietermaritzburg (Burnup). Common throughout Natal.

Lorenzo Marques. Delagoa Bay (Connolly).

235. Trachycystis calorama, Melv. \& Pons. [S.A.M.] 1899 Trachycystis calorama, M.\&P., A.M.N.H.iv.p. 196.pl.3, f.6. D.F. Type in British Museum.

Hab. Natal. Pinetown; Inchanga (Burnup).

236. Trachycystis centrifuga, Melv. \& Pons. [S.A.M.] 1903. Trachycystis centrifuga, M. \& P., A.M.N.H. xii. p. 602. pl. 32, f. 9. D.F.

Type in British Museum.

Hab. Cape of Good Hope. Maeström Forest, Bedford (Farquhar). Pirie Forest (var., Godfrey).

237. Trachycystis charybdis (Benson).

[S.A.M.]

1856 Helix charybdis, Bs., A.M.N.H. xviii. p. 436. D.

1859 " " Pfr., Mon. Hel. iv. p. $106 . \quad D$.

$1899 \quad$ ", $\quad$ M.\&P, A.M.N.H.iv.p. 194.pl.3, f.16. N.F.

Specimen ex auct. in University Museum of Zoology, Cambridge.

Hab. Cape Peninsula. Table Mountain (Layard; Lightfoot; Connolly). Kalk Bay (Lightfoot). 
238. Trachycystis conisalea (Melv. \& Pons.). [S.A.M.]

1892 Helix (Pella) conisalea, M.\&P., A.M.N.H.x.p.239. pl.13, f.7. D.F. 1893 Phasis (Trachycystis) conisalea, M. \& P., Pilsb., Man. of Conch. viii. p. 145: pl. 35 , f. 4 . D.F.

Type in British Museum.

Hab. NataL. Pietermaritzburg; Nottingham Road; Karkloof; Dargle; Equeefa; Table Mountain, E. of Pietermaritzburg (Burnup).

239. Trachycystis connollyi, Melv. \& Pons. [S.A.M.] 1909 Trachycystis connollyi, M.\&P., A.M.N.H.iv.p.491.pl.8,f.15. D.F. Type in British Museum.

Hab. Cape of Good Hope. Montagu (Connolly). Kuruman (in coll. Layard).

Nearly allied to $T$. farquhari (M. \& P.).

240. Trachycystis coxi, Preston.

1912 Trachycystis coxi, Prest., Proc. Mal. Soc. x. p. 18. D.F.

Type in coll. Dautzenberg.

Hab. Cape of Good Hope. Knysna Forest (Cox).

Resembles inclara, Morelet, from which it differs in being imperforate and of a more delicate substance.

241. Trachycystis ectima, Melv. \& Pons.

1899 Trachycystis ectima, M. \& P., A.M.N.H.iv. p. 197.pl.3, f.8. D.F. Type in British Museum.

Hab. NataL. Umkomaas; Tongaat (Burnup).

242. Trachycystis epetrima (Melv. \& Pons.).

1892 Helix (Pella) epetrima, M. \& P., A.M.N.H.ix.p.84.pl.4, f.3. D.F. 1893 Phasis (Trachycystis) epetrima, M. \& P., Pilsb., Man. of Conch. viii. p. 146. pl. 35 , f. 6 . D.F.

Type in British Museum.

Hab. Cape of Good Hope. Somerset District (fide M. \& P.). North End, Port Elizabeth (Crawford).

243. Trachycystis erythractis, sp. nov., pl. 2, f. 5. [S.A.M.]
[S.

Shell depressed-conic, subrimate, rather thin and dull, hardly translucent; with close, faint, regular striation parallel to the curve of the outer lip; pale cream-coloured, prettily marked on the upper side with irregular ruby rays and streaks; the underside, though 
similarly striate, is of thinner texture and devoid of red marking.

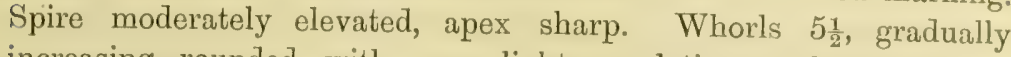
increasing, rounded, with very slight angulation at the periphery. Suture simple, shallow. Aperture compressed oval; peristome thin, simple; columella short, thickened upwards, margin narrowly reflexed, almost entirely concealing the perforation.

Diam. maj. $10 \cdot 1$, min. 8.9 ; alt. 6.8 ; Apert. alt. $5 \cdot 2$, lat. $5.4 \mathrm{~mm}$.

Type in South African Museum.

Hab. CAPE OF Good Hope. Caledon Division (Lightfoot).

Possibly nearer Phortion than Trachycystis, but differing therefrom in one or two important particulars.

244. Trachycystis farquhari (Melv. \& Pons.).

1892 Helix farquhari, M.\& P., A.M.N.H. x. p. 240. pl.13, f.9. D.F. 1893 Phasis (Trachycystis) farquhari, M. \& P., Pilsb., Man. of Conch. viii. p. 147. pl. 35 , f. 12 . D.F.

Type in British Museum.

Hab. CAPE OF Good Hope. Port Elizabeth (Farquhar).

Very nearly allied to $T$. connollyi, from which it possibly differs in having rather less convex whorls and more concave columella.

\section{Trachycystis glanvilliana (Ancey).}

1890 Helix glanvilliana, Ancey, Bull. Soc. Mal. Fr. vii. p. 157. D. 1893 Phasis (Trachycystis) glanvilliana, Ancey, Pilsb., Man. of Conch. viii. p. 147. N.

Type in coll. Geret.

Hab. Cape of Good Hope. Grahamstown (Miss Glanville).

246. Trachycystis glebaria, Melv. \& Pons.

1903 Trachycystis glebaria,M.\& P., A.M.N.H.xii.p.602.pl.32,f.15. D.F. Type in British Museum.

Hab. NataL. Pinetown; Hilton Road (Burnup).

\section{Trachycystis hartvigiana (Pfeiffer).}

1861 Helix hartvigiana, Pfr., Mal. Blätt. viii. p. 167. D.

$1868 \quad$ " 1886 Mon. Hel. v. p. 239. D.

$1886 \quad$ " Kob., Conch. Cab. p. 617. pl. 178, f. 10 12. D.F.

1887 Helix (Pella) hartvigiana, Pfr., Tryon, Man. of Conch. iii. p. 107. pl. 20, f. 89-91. D.F.

1898 Pella hartvigiana, Pfr., Stur., S,A, Moll. p. 39. N, 
Type in Stettin Museum.

Hab. Cape of Good Hope. Gnadenthal, near Stellenbosch (Hartvig). Oudebosch (Layard).

248. Trachycystis hottentota (Melv. \& Pons.). [S.A.M.] 1891 Helix hottentota, M. \& P., A.M.N.H. viii. p. 239. D.

$1892 \quad$ " " " ix. p. 94. pl. 4, f. 6. F.

1893 Plasis (Trachycystis) hottentota, M. \& P., Pilsb., Man. of Conch. viii. p. 141. pl. 35, f. 8 . D.F.

1907 Trachycystis paula, M. \& P., A.M.N.H.xix.p.99.pl.6,f.11. D.F.

Types in British Museum.

Hab. Cape of Good Hope. Happy Valley and Rufane Vale, Port Elizabeth (Crawford). Wynberg; Simonstown; Lakeside; Caledon (Connolly).

Natal. Pietermaritzburg (Penther). Majuba (Connolly). Game Pass, \&c. (Burnup).

Transvadu. Johannesburg (paula, McBean). Pretoria ; Pienaars Poort; Buiskop (Connolly).

A somewhat variable species, in which the beautiful distant costulate sculpture, so noticeable in immature or very fine adult specimens, disappears quickly when exposed to wear. The type of paula has been very carefully compared with that of hottentota, and is undoubtedly conspecific.

249. Trachycystis inclara (Morelet).

1889 Helix inops, Morel., J. de C. xxxvii. p. 6. pi. 1, f. 2. D.F.

, , inclara, Morel., (=inops, Morel., 1889, nec Mouss., 1872),

Morel., J. de C. xxxvii. p. 200.

, , bowkeriana, Ancey, in litt.

1893 Phasis (Trachycystis) inops, Morel., Pilsb., Man. of Conch. viii. p. 144 . pl. 43 , f. 50,51 . D.F.

Type in British Museum.

Hab. Cape of Good Hope. Port Elizabeth; Alexandria District (Crawford). Grahamstown (boukcrianc, Miss Glanville). Tharfield (bowticriana, Miss Borvker). Bedford; Bathurst (Farquhar). Port St. John's (Shortridge).

250. Trachycystis hnisnaensis, Preston.

1912 Trachycystis linysnaensis, Prest., Proc. Mal. Soc. x. p. 17. D.F. Type in coll. Dautzenberg.

Hab. Cape of Good Hope, Knysna Forest (Cox). 
251. Trachycystis uaticostata, Melv. \& Pons.

1903 Trachycystis laticostata, M. \& P., A.M.N.H. xii. p. 602. pl. 32, f. 5. D.F.

Type in British Museum.

Hab. CAPE of Good Hope. Maeström Forest, Bedford (Farquhar).

252. Trachyorstis lignicola, Melv. \& Pons.

1898 Trachycystis lignicola, M. \& P., A.M.N.H.ii.p.125.pl.7, f.1. D.F. Type in British Museum.

Hab. Cape of Good Hope. Fish River, Cradock (Farquhar).

253. Trachycystis irricostata (Melv. \& Pons.).

1891 Helix (Pella) liricostata, M. \& P., A.M.N.H. viii. p. 239. D. $1892 \quad$ " " $\quad$ "ix.p.94.pl.5,f.1. $F$. 1893 Phasis (Trachycystis) liricostata, M. \& P., Pilsb., Man. of Conch. viii. p. 140. pl. 35, f. 9. D.F.

Type in British Museum.

Hab. Cape of Good Hope. East Griqualand (fide M. \& P.). Van Staadens River and Coerney, Port Elizabeth (Crawford).

\section{Trachycystis Lovéni (Krauss).}

1848 Helix lovéni, Krs., Südafr. Moll. p. 76. pl. 4, f. 21. D.F.

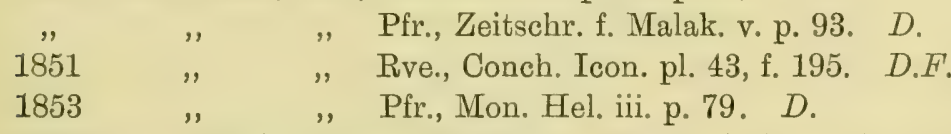

1887 Helix (Pella) loveni, Krs., Tryon, Man. of Conch. iii. p. 106. pl. 20, f. 74. D.F.

1898 Phasis (Trachycystis) loveni, M. \& P., Stur., S.A. Moll. p. 45. (Err. typ.)

Type in Stuttgart Museum.

Hab. NataI (Wahlberg).

255. Trachycystis LYGEA (Melv. \& Pons.). [S.A.M.] 1892 Helix (Pella) lygaa, M. \& P., A.M.N.H. ix. p. 85. pl. 4, f. 7. D.F. 1893 Phasis (Trachycystis) lygaa, M. \& P., Pilsb., Man. of Conch. viii. p. 138. pl. 35, f. 7. D.F.

1899 Trachycystis lygaa, M. \& P., Moss \& Webb, Proc. Mal. Soc. iii. p. $263 . A$.

Type in British Museum.

Hab. Natal. Pietermaritzburg (Burnup).

Cape of Good Hope. Somerset East District (Miss Bowker). 
256. Trachycystis metallaiter, sp. nov., pl. 2, f. 6. [S.A.M.]

Shell small, depressed globose, umbilicate, thin, fulvo-corneous, semitransparent. Spire little raised, apex rounded. Whorls 4, convex, gradually increasing; beautifully sculptured above on the second, third, and part of the fourth with delicate, curved, rather distant, transverse liræ, which are clear, but less pronounced, on the underside; towards the aperture the striation becomes closer and fainter. Suture deep. Aperture sub-ovate, descending slightly; peristome thin, simple. Umbilicus not very wide, but deep, extending to the apex. Columella weak, margin slightly reflexed, but in no way concealing the umbilicus.

Diam. maj. 4.0 , min. 3.4 ; alt. 2.2 ; apert. alt. 1.9 , lat. $1.5 \mathrm{~mm}$.

Type in British Museum.

Hab. Cape of Good Hope. Caledon (Connolly).

As with the far smaller hottentota, M. \& P., the distant liræ, which are such a beautiful feature in immature shells, wear off with increasing age, and do not seem to be produced after the first $3 \frac{1}{2}$ whorls. I have chosen the type carefully, as being in a transitional stage, for specimens of exceptional size possessing 5 whorls show hardly a trace of the early sculpture, and might well be mistaken for a different species.

\section{Trachycystis microscopica (Krauss).}

1848 Helix microscopica, Krs., Südafr. Moll. p. 76. pl. 4, f. 20. D.F. " " " Pfr., Zeitschr. f. Malak. v. p. 93. D. $1853 \quad$ " $\quad$ " , Mon. Hel. iii. p. 83. D.

1887 Helix (Pella) microscopica, Krs., Tryon, Man. of Conch. iii. p. 106. pl. 20, f. 77, 78. D.F.

Type in Stockholm Museum.

Hab. Natal. Mooi River (Wahlberg).

Through the courtesy of Dr. S. Théele, of Stockholm, I am enabled to give, on p. 159, a photograph of this little-known species. It will be seen that it can hardly be placed in Pupisoma, but its exact generic position is extremely doubtful.

258. Trachycystis mirostriata, Preston.

1912 Trachycystis microstriata, Prest., Proc. Mal. Soc. x. p. 18. D.F. Type in coll. Dautzenberg.

Hab. Cape of Good Hope. Knysna (Cox). 
259. Trachycystis ordinaria, Melv. \& Pons.

[S.A.M.]

1908 Trachycystis ordinaria, M.\& P., A.M.N.H.i.p.135.pl. 7, f.8. D.F.

Type in British Museum.

Hab. Transvaal. Potchefstroom (Miss Livingston).

Orange Free State. Bloemfontein; Rustfontein (Connolly). Kroonstad (Miss Hickey).

Specimens from Rustfontein have rather coarser and more distant striation than the typical form, but are hardly specifically separable.

260. Trachycystis oreina, Melv. \& Pons.

1903 Trachycystis oreina, M. \& P., A.M.N.H. xii.p.602.pl.32,f.8. D.F. Type in British Museum.

Hab. Cape of Good Hope. Grahamstown (Farquhar).

261. Trachycystis patera, Melv. \& Pons.

1903 Trachycystis patera, M.\&P., A.M.N.H.xii.p.603.pl. 32, f. 6. D.F.

Type in British Museum.

Hab. Cape of Good Hope. Maeström Forest, Bedford (Farquhar). Simonstown (Connolly).

262. Trachycystis permeata, Melv. \& Pons.

1903 Trachycystis permeata, M.\&P.,A.M.N.H.xii.p.603.pl.32,f.2. D.F . Type in British Museum.

Hab. Natal. Pinetown; Tongaat (Burnup).

263. Trachycystis perplicata (Benson).

1851 Helix perplicata, Bs., A.M.N.H. vii. p. 104. D.

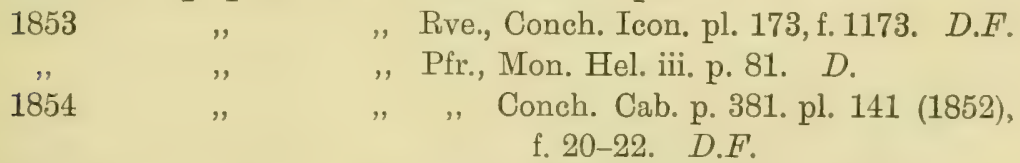

1885 Helicopsis tabute, Chaper, Bull. Soc. Zool. Fr. х. p. 483. pl. 11, f. 4,5 . D.F.

1887 Helix (Pella) perplicata, Bs., Tryon, Man. of Conch. iii. p. 106. pl. 20, f. 76. D.F.

$1892 \quad$ " $\quad$ rhysodes, M.\&P., A.M.N.H.ix.p. 87.pl.4, f.2. D.F.

1893 Phasis (Trachycystis) rhysodes, M. \& P., Pilsb., Man. of Conch. viii. p. 141. pl. 35, f. 2. D.F.

tabula, Chaper, Pilsb., Man. of Conch. viii. p. 139. pl. 43 , f. 52 . D.F.

Specimens of perplicata, ex auct., in University Museum of 
Zoology, Cambridge. Type of rhysodes in British Museum; tabule in École des Mines, Paris.

Hab. Cape of Good Hope. Near Newlands (Benson). Table Mountain (tabula, Chaper). ? Port Elizabeth (fide Sturany). Widely distributed throughout the Cape Peninsula from Table Mountain to Cape Point.

The type of rhysodes, from the Layard collection, from an unknown locality, is identical with perplicata, Bs.; and after careful study of Chaper's description and figure of tabula, I have no hesitation in placing the latter in the same synonymy.

\section{Trachycystis petrobia (Benson).}

1851 Helix petrobia, Bs., A.M.N.H. vii. p. 105. D.

1853 " „ Pfr., Mon. Hel. iii. p. $95 . \quad D$.

" $\quad$ " $\quad$ " Rve., Conch. Icon. pl. 173, f. 1169. D.F.

1854 ," , Pfr., Conch. Cab. p. 382. pl. 141 (1852), f. 25-27. D.F.

1887 Helix (Pella) petrobia, Bs., Tryon, Man. of Conch. iii. p. 107. pl. 20, f. 81. D.F.

Hab. Cape of Good Hope. High Constantia (Benson). Port Elizabeth, North End (Crawford).

\section{Trachycystis Pinguis (Krauss).}

1848 Helix pinguis, Krs., Südafr. Moll. p. 75. pl. 4, f. 19. D.F.

, " " " Pfr., Zeitschr. f. Malak. v. p. $91 . \quad D$.

1851 ", " Rve., Conch. Icon. pl. 43, f. 200. D.F.

$1853 \quad$ " " Pfr., Mon. Hel. iii. p. 64. D.

1887 Helix (Pella) pinguis, Krs., Tryon, Man. of Conch. iii. p. 103. pl. 20 , f. 60 . D.F.

1898 Pella pinguis, Krs., Stur., S.A. Moll. p. 39. N.

Type in Stuttgart Museum.

Hab. NataL (Wahlberg).

No recent localities are given, as doubt exists whether the shell, which has of late years been generally accepted as pinguis, is in reality that species. von Martens' record of Tette must also be viewed with distrust.

266. Trachycystis planti (Pfeiffer).

1854 Helix platti, Pfr., P.Z.S. p. 51. (Err. typ.) D.

, , planti ," Rve., Conch. Icon. pl. 189, f. 1325. D.F'.

$1859 \quad$ " " " Mon. Hel. iv. p. 60. D. 
1885 Helix planti Pfr., Kob., Conch. Cab.p. 622.pl.178, f. 29-31. D.F. 1887 „, (Pella) planti, Pfr., Tryon, Man. of Conch. iii. p. 105. pl. 20 , f. 73. D.F.

1893 Phasis (Trachycystis) planti, Pfr., Pilsb., Man. of Conch. viii. p. 142. pl. 43, f. 57-59. N.F.

Type in British Museum.

Hab. Natal (Plant). Lower Umkomaas; Durban; Tongaat (Burnup).

Zululand, Umbonambi (Burnup).

? Transvaal. Lydenburg (fide Craven). A very doubtful locality. CAPE OF Good Hope. Tharfield, near Kleinmont Rivermouth (Miss Bowker, fide Layard).

\section{var. AFrice, Brown.}

1865 Helix africa, Brown, Amer. Journ. of Conch. i. p. 136. D. $1876 \quad$ " " Pfr., Mon. Hel. vii. p. 459. D.N.

$1877 \quad$ " " " Conch.Cab.p.527.pl.162,f.8, 9. D.F. 1893 Phasis (Trachycystis) africa, Brown, Pilsb., Man. of Conch. viii. p. 142. pl. 43, f. 55, 56. D.F. planti, Pfr., var. africa, Brown, Pilsb. Man. of Conch. ix. p. 38.

Type in Philadelphia Museum.

Hab. Cape of Good Hope. Great Brakke (Brown).

267. Trachycystis prionacis (Benson).

1864 Helix prionacis, Bs., A.M.N.H. xiii. p. 493. D.

. ". ", browningii ," , , , D.

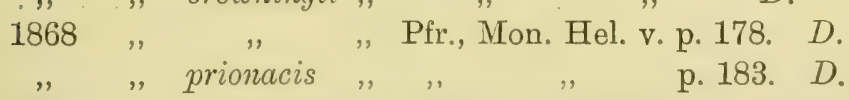

1892 " (Pella) erateina, M. \& P., A.M.N.H.ix.p. 88. pl.5, f. 3. D.F. 1893 Phasis (Trachycystis) erateina, M. \& P., Pilsb., Man. of Conch. viii. p. 137. pl. 35 , f. 11 . D.F. prionacis, Bs., Pilsb., ibid. p. 137. D. browningii, Bs., Pilsb., ibid. p. $136 . \quad D$. browningii, Bs., Pilsb., Man. of Conch. ix. p. 38. pl. 10, f. 8, 9. F.

1898 Trachycystis eratina, M. \& P., Proc. Mal. Soc. iii. p. 173.

Type of eratina in British Museum; originals of browningii in University Museum of Zoology, Cambridge; prionacis probably in British Museum.

Hab. CAPE of Good Hope. Bredasdorp (prionacis, Layard). 
Noar Cape Point (browningii, Browning). Bredasdorp ; Cape Point (eratina, Layard).

Prionacis was founded on a single specimen furnished by Layard from Bredasdorp; browningii on two found by Browning near Cape Point; eratina on shells in coll. Layard from Bredasdorp and Cape Point.

In the recently dispersed Layard collection was a tube labelled "erateina" containing shells from Cape Point and Bredasdorp. These were identical with original browningii in the Cambridge Museum. Prionacis was differentiated by Benson from browningii on account of larger size, less close plication, extending further down on the underside, and wider umbilicus. All these points are consistent with the increasing growth of the shell, young examples of which are almost imperforate, and show no basal sculpture. It seems advisable to unite the three species, of which prionacis has priority.

268. Trachycystis pycnotricha, Melv. \& Pons.

1899 Trachycystis pycnotricha,M.\&P.,A.M.N.H.iv.p.197.pl.3,f.7 . D.F. Type in British Museum.

Hab. Cape of Good Hope. Kowie River (Cox).

269. Trachycystis rariplicata (Benson).

1849 Helix rariplicata, Bs.in Mss., Pfr., Zeitschr.f. Malak. vi.p.71. D. 1850

1853 , " Pfr., Conch. Cab. p. 323. pl. 129 (1852), , A.M.N.H. vi. p. 254. D. f. 31-33. D.F.

" $\quad$ " $\quad$ "Rve., Conch. Icon. pl. 174, f. 1183. D.F. " " " Pfr., Mon. Hel. iii. p. $96 . \quad D$.

1879 Helix (Pella) rariplicata, Bs., Binn., Ann. N.Y. Acad. Sci. i. p. 361. pl. 14, f. H. R.

1884 Pella rariplicata, Bs., Binn., Ann. N.Y. Acad. Sci. iii. p. 89. pl. 3, f. I. $R$.

1887 Helix (Pella) rariplicata, Bs., Tryon, Man. of Conch. iii. p. 107. pl. 20, f. 82. D.F.

Type in Stettin Museum.

Hab. Cape Peninsula. Green Point Lighthouse (Benson; Layard).

Examples presented to the British Museum as rariplicata by Gibbons in 1876 have proved to be not that species, but sabuletorum. As Binney obtained his specimens from Gibbons, his articles in all probability refer to the last-mentioned species. 
270. Trachycystis rivulakis (Krauss).

1848 Helix rivularis, Krs., Südafr. Moll. p. 77. pl. 4, f. 25. D.F.

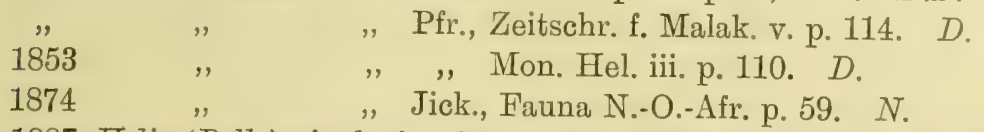

1887 Helix (Pella) rivularis, Krs., Tryon, Man. of Conch. iii. p. 107. pl. 20, f. 85-87. D.F.

Type in Stockholm Museum.

Hab. NataL. Source of Mooi River (Wahlberg).

var. DENSESTRIATA, nov.

Shell small, rather depressed, umbilicate, thin, bright fulvo-corneous, covered on both sides with close, prominent, regular, curved,
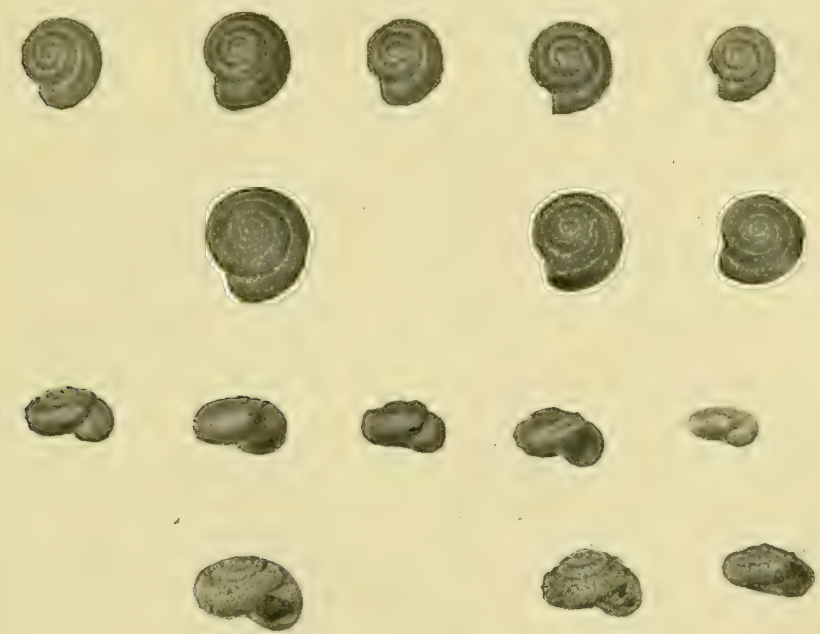

3

$$
2
$$

1

5

4

1. Trachycystis rivularis, Krs. (Type).

2. ", " var. densestriata, Zwart Kop.

3. " " " " Pietersburg.

4. ", ordinaria, M. \& P., Bloemfontein.

5. ", " " lirate var., Rustfontein.

transverse striæ. Whorls $4 \frac{1}{2}$, rounded, gradually increasing. Suture impressed. Aperture lunate, descending a little in front; peristome thin, simple; columella weak, concave, margin slightly reflexed, but in no way covering the umbilicus, which, though not wide, is deep, extending to the apex. 
Diam. maj. 4.5 ; min. 3.5 ; alt. 2.2 ; Apert., lat. 1.8 , alt. $1.6 \mathrm{~mm}$. Type in British Museum.

Hab. Transvaal. Zwart Kop, Pretoria; Buiskop; Pietersburg (Connolly).

The foregoing photographs give some idea of the form of rivularis and its variety, compared with ordinaria, M. \& P. Densestriata differs from Krauss' type in having slightly, though very noticeably, closer striation, but agrees with it in all other respects, and does not appear to be specitically separable.

Nobre has recently recorded rivularis from Angola, but this is unlikely to be correct.

271. Trachycystis rutilans, Melv. \& Pons. [S.A.M.] 1908 Trachycystis mutilans, M.\& P., A.M.N.H. i.p. 135. pl.7, f.9. D.F. Type in British Museum.

Hab. Natal. Alexandra Park, Pietermaritzburg (Burnup).

272. Trachycystis sabuletorum (Benson). [S.A.M.]
[S.

1851 Helix sabuletorum, Bs., A.M.N.H. vii. p. 105. D.

1853

„ Pfr., Mon. Hel. iii. p. $96 . \quad D$.

" $\quad$ " $\quad$ Rve., Conch. Icon. pl. 174, f.1180. D.F.

1854 ", , Pfr., Conch. Cab. p. 414. pl. 147 (1853), f. 16, 17. D.F.

1887 Helix (Pella) sabuletorum, Bs., Tryon, Man. of Conch. iii. p. 107. pl. 20, f. 83 . D.F.

Specimens ex auct. in University Museum of Zoology, Cambridge.

Hab. Cape of Good Hope. Hout Bay; Strand not far from Somerset; Kalk Bay (fide Benson). Stumpnose (Gould). Hermanus (Lightfoot). Generally distributed along the coast of the Cape Peninsula.

273. Trachycystis scolopendra, Melv. \& Pons. [S.A.M.] 1903 Trachycystis scolopendra, M. \& P., A.M.N.H. xii. p. 603. pl. 32, f. 3. D.F.

Type in British Museum.

Hab. Natal. Port Shepstone; Equeefa; Durban; Lower Umkomaas (Burnup). Umzimkulu (Purcell).

Cape of Good Hope. Port St. John's (Shortridge). Pirie (Godfrey). 
274. Trachycystis smplex, Melv. \& Pons.

1903 Trachycystis simplex, M.\&P.,A.M.N.H.xii.p.604. pl.32,f.7. D.F. Type in British Museum.

Hab. Cape of Good Hope. Pondoland (Farquhar).

275. Trachycystis somersetensis (Melv. \& Pons.).

1893 Helix (Patula) somersetensis, M. \& P., A.M.N.H. xi. p.19. pl. 3, f. 2. D.F.

", , , , Pilsb., Man.of Conch.viii.

Type in British Museum. p. 295.pl. 43, f.63. D.F.

Hab. Cape of Good Hope. Somerset East (Miss Bowker).

276. Trachycystis spissicosta, Melv. \& Pons, [S.A.M.] 1907 Trachycystis spissicosta, M. \& P., A.M.N.H. xix. p. 100. pl. 6, f. 13. D.F.

Type in British Museum.

Hab. CAPE of Good Hope. Grahamstown (Farquhar).

Orange Free State. Thaba N'chu (Connolly).

Rather near, but smaller than, T. simplex, M. \& P.

277. Trachycystis teretiuscula, Melv. \& Pons.

1897 Trachycystis teretiuscula, M. \& P., A.M.N.H. xix. p. 635. pl. 17, f. 5. D.F.

Type in British Museum.

Hab. Natal. Howick; Pietermaritzburg; Equeefa (Burnup). Majuba (Connolly).

Orange Free State. Platberg, Harrismith (Connolly).

Cape of Good Hope. East London (var.) (Godfrey).

A very variable species.

278. Trachycystis toldini (Albers.).

1855 Nanina afra, Pfr., Mal. Blätt. ii. p. 119. (Without characters.)

1856 Helix tollini, Alb., Bs., A.M.N.H. xviii. p. 436. D.

1857 " " " Mal. Blätt. iv. p. 94. D.

$1859 \quad$, $\quad$ " $\quad$ " Pfr., Mon. Hel. iv. p. 171. D.

, " $\quad$ afra, Pfr., Mon. Hel. iv. p. 124. D.

1860 " roseri, Krs., von Mts., Die Helic., p. 84. L.

1886 " tollini, Alb., Kob., Conch. Cab.p. 618.pl.178, f. 13-15. D.F.

1887 " (Pella) tollini, Alb., Tryon, Mian. of Conch. iii. p. 108. pl. 21, f. 92-94. D.F. 
1892 Helix (Pella) tuguriolum, M. \& P., A.M.N.H. ix. p. 88. pl. 5, f. 5. D.F.

1893 Phasis (Trachycystis) tuguriolum, M. \& P., Pilsb., Man. of Conch. viii. p. 145. pl. 35, f. 1. D.F.

1898 Pella tollini, Alb., Stur., S.A. Moll. pp. 39, 40. N.

Originals of tollini in University Museum of Zoology, Cambridge ; types of afra and tuguriolum in British Museum; roseri in Stuttgart Museum.

Hab. Cape of Good Hope (afra, MeGillivray, fide Pfeiffer). Table Mountain (tollini, Tollin; Layard). Stellenbosch (Purcell). Kalk Bay; Simonstown; Kommetje; Houw Hoek; Caledon (Connolly). Gordon's Bay; Hermanus (Lightfoot). Bredasdorp (Layard). Swellendam District (roseri, Krauss).

Helix afra, Pfr., is identical with tollini, Alb., and must yield priority to the latter, which was the earliest name under which the species was either described or figured. The type set of tuguriolum, labelled "South Africa" (coll. Layard), are also immature, rather pale examples of the same species.

The type, and only authentic example, of roseri, Krauss, is a semibleached shell, in form exactly resembling immature tollini. Its colour is paler, and the surface, being considerably worn, shows only the cross striation of tollini and not the fainter spiral sculpture. The columellar fold is slightly broken, which gives a more open appearance to the umbilical region than is usual in tollini, but the underside of the shell and the peculiar shape of the peristome agree with that species. After careful consideration, I think I may safely declare them to be one and the same.

279. Trachyctstis trichostiroma (Melv. \& Pons.).

1892 Helix (Pella) trichosteiroma, M. \& P., A.M.N.H. ix. p. 84. pl. 4, f. 9 . D.F.

$1893 \quad$ " strobilodes, M.\&P., A.M.N.H.xi.p.19.pl.3,f.1. D.F.

" Phasis (Trachycystis) strobilodes, M. \& P., Pilsb., Man. of Conch. viii. p. 147. pl. 43, . 62 . D.F.

" , trichosteiroma, M. \& P., Pilsb., Man. of Conch. viii. p. 143. pl. 35, f. 3. D.F.

1898 Trachycystis trichostiroma, M. \& P., Proc. Mal. Soc. iii. p. 174. Types in British Museum.

Hab. Cape of Good Hope. Port Elizabeth (fide M. \& P.). Tharfield (Miss Bowker).

The type of strobilodes is badly figured, but agrees with the original description. It is quite inseparable from trichostiroma. 
280. Trachycystis turmatis (Morelet).

1889 Helix turmalis, Morel., J. de C. xxxvii. p. 5. pl. 1, f. 1. D.F.

1893 Phasis (Trachycystis) turmalis, Morel., Pilsb., Man. of Conch. viii. p. 144. pl. 43, f. 53, 54. D.F.

Type in British Museum.

Hab. CAPE of Good Hope. Zwartkops River, Uitenhage, 19 miles N.N.W. of Port Elizabeth (Crawford).

\section{Trachycystis vorticialis (Benson). [S.A.MI.]}

1850 Helix vorticialis, Bs., A.M.N.H. v. p. 216. D.

1853 " " " " Pfr., Mon. Hel. iii. p. 108. D.

" " " , " Rve., Conch. Icon. pl.174, f. 1181. D.F.

1854 ", vorticalis ", Pfr., Conch. Cab. p. 382. pl. 141 (1852), f. 28-30. D.F. (pessina).

1887 "(Pella) vorticialis, Bs., Tryon, Man. of Conch. iii. p. 107. pl. 20, f. $84 . \quad$ D.F.

Type in British Museum.

Hab. Cape Peninsula. Three Anchor Bay; Rondebosch; Strand, False Bay (fide Benson). "Generally distributed" (Layard).

? CAPE OF Good Hope. Port Elizabeth (fide Sturany).

FAmily ACAVID $æ$, Pilsbry, 1902.

(Man. of Conch. xiv. p. iv.)

(= Macroogona, Pilsbry, 1895.)

Genus DORCASIA, Gray, 1838.

(Alexander's Expedition, ii. p. 268.)

Type of Genus, D. alexandri, Gray.

282. Dordasia alexandri, Gray.

[S.A.M.]

1838 Dorcasia alexandri, Gray, Alexander's Expedition, ii. p. 268, D. 1845 Helix (Dorcasia) alexandri, Gray, Pfr., Zeitschr.f. Malak.p. 87. D. $1848 \quad$ " $\quad, \quad$ Mon. Hel. i. p. 332. D. 1854 " alexandri, Gray, Rve., Conch. Icon. pl. 208, f. 1470. D.F. $1877 \quad$ " " $\quad$ " Pfr., Novit. Conch. v. p. 34. pl. 144, f. 9-12. D.F.

1887 "(Dorcasia) alexandri, Gray, Mouss., J. deC.xxxv. p. 292. N. " ," „, Tryon, Man. of Conch. iii. p. 213. pl. 49, f. 3. D.F. ", Srth., Ber. Senckenb. Naturf. Ges. Frankfurt, p. 94. A. 
1895 Dorcasia alexandri, Gray, Pilsb., Man. of Conch. ix. p. 172 pl. 60, f. 6. R. Frontisp. f. 3. $A$.

1905 Pilsb., Proc. Mal. Soc. vi. p. 286. $N$. 1910 Helix (Dorcasia) alexanderi, Gray, Bttg., Abh. Senckenb. Naturf. Ges. Frankfurt, xxxii. p. 439. N.

Type in British Museum.

Hab. Great Namaland. Near the Great Fish River (Alexander). Damaraland. Ussab (fide von Martens). "Kurikaubmund am Swakop (Kurikop bei Otjikango)" (Rintelen).

Littie Namaland. Henkries (Lightfoot).

var. MINoR, Böttger.

1886 Helix (Dorcasia) alexandri, Pfr., var. minor, Bttg., Ber. Senckenb. Naturf. Ges. Frankfurt, p. 22. pl. 2, f. 1. D.F.

1893 Dorcasia alexandri, Gray, var. minor, Bttg., Pilsb., Man. of Conch. viii. p. 261. pl. 28, f. 98-100. D.F.

Type in Senckenberg Museum, Frankfurt.

Hab. British Bechuanaland. "Ghous, Gordonia, bed of Hygap, probably W. of Zwart Modder" (Nolte).

Great Namaland. Geitsi-Gubel, near Bersaba (Schenck).

var. Rotundata, Mousson.

1887 Helix alexandri, Gray, var. rotundata, Mouss., J. de C. xxxv. p. 292. pl. 12, f. 1 . D.F.

1893 Dorcasia alexandri, Gray, var. rotundata, Mouss., Pilsb., Man. of Conch. viii. p. 261. D.

1895 Dorcasia alexandri, Gray, var. rotundata, Mouss., Pilsb., Man. of Conch. ix. p. 172. pl. 38, f. 6, 7. N.F.

Type in Zurich Museum.

Hab. Damaraland. Rehoboth (Schinz).

$$
\text { var. TRIvia, Böttger. }
$$

1910 Helix (Dorcasia) alexanderi, Gray, var. trivia, Bttg., Abh. Senckenb. Naturf. Ges. Frankfurt, xxxii. p. 439. pl. 28, f. 3. D.F. Type in Senckenberg Museum, Frankfurt.

Hab. Damaraland. Khomas-Hochland (Schultze).

\section{Dorcasia Bulbus (Menke).}

1848 Helix bulbus, Mke., Pfr., Zeitschr. f. Malak. v. p. 116. D.

1853 " " ", Conch. Cab. p. 268. pl. 122 (1852), f. $4-6 . D \cdot F$. 
1887 Helix (Dorcasia) bulbus, Pfr., Tryon, Man. of Conch. iii. p. 213.

Type-ubi?

pl. 49, f. 10-12. D.F.

Hab. Cape of Good Hope (in coll. Menke).

\section{Dorcasia cernua (von Martens).}

1889 Helix cermu, von Mts., Sitz.-Ber. Ges. Nat.Fr. Berlin, p. 161. D. 1894

1897

Conch. Mitth. iii, 3. p. 2. D.

Archiv. f. Naturg. Ixiii, 1.p. 36.pl. 7, f.810. D.F.

1910 Helix (Dorcasia) cermua, von Mts., Bttg., Abh. Senckenb. Naturf.

Ges. Frankfurt, xxxii. p. 439 . N.D.

Type in Zool. Mus. Berlin.

Hab. Great Namaland. Angamthal (type) and Rooiberg, near Bethany (Schenck, 1884). Guibes (Schultze).

\section{Dorcasia lucana (Müller).}

1774 Helix lucana, Müll., Verm. ii. p. 75. D.

1786 " " , " Chem., Conch. Cab. ix, 2. p. 124. pl. 130, f. 1155 . D.F. (pessima).

1788. "lucena ", Gmel., Syst. Nat., Ed. 13. i, 6. p. 3636. D. 1817 ", lucana " Dillw., Descr. Cat. ii. p. 924. D.

$1839 \quad$ " , Jonas, Archiv. f. Naturg. v, 1. p. 338. pl. 9, f. 1, 2. D.F.

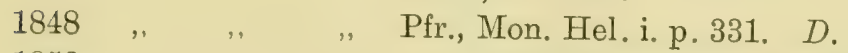

$1850 \quad$ " $\quad$, $\quad$, , Conch. Cab. p. 338.pl.60, f. 1, 2. D.F.

" " " Desh., Hist. Nat. Moll. i. p. 166. pl. 10 B, f. 3-5. D.F.

$1851 \quad, \quad$, $\quad$ " Rve., Conch. Icon. pl. 41, f. 187. D.F.

$1856 \quad$ " , " $\quad$ Bs., A.M.N.H. xviii. p. $436 . \quad$ N.

1887 ", (Dorcasia) lucana, Müll., Tryon, Man. of Conch. iii. p. 213. pl. 49, f. 2. D.F.

Original in University Zool. Mus. Copenhagen.

Hab. CAPE of Good Hope. George District; Bredasdorp; Mossel Bay (Layard). Montagu (Connolly). Avontuur (fide Pfr.).

Cape Peninsula. Distributed along the coast south of Kalk Bay and Hout Bay.

Layard wrote: "This species is found chiefly along the southern seaboard. The brown variety with white band along the suture is found pretty abundantly about Kalk Bay. A smaller var. with a brownish purple mouth is found in the George District; a small var. 
(axis 6"', diam. $8^{\prime \prime \prime}$ ) with a white mouth is not uncommon at Bredasdorp, while a large white form (axis $1^{\prime \prime}$, diam. $1^{\prime \prime} 3^{\prime \prime \prime}$ ) exists at Mossel Bay."

Chemnitz's figure (1786) is almost unrecognisable, while Lamarck (1822) described globulus for lucana, and Rossmässler (1837) figured globulus as the last-mentioned species. Krauss (1848) included lucana, Lam., in the synonymy of globulus, Müll., but unaccountably omitted lucana, Müll., altogether from his catalogue.

286. Dorcasia ponsonbyi, Fulton.

1910 Dorcasia ponsonbyi, Fulton, A.M.N.H. vi. p. 212. D.

Type in British Museum.

Hab. Cape of Good Hope (fide Fulton). Mossel Bay (Gibbons).

Sub-Genus TULBAGHINIA, Melv. \& Pons., 1898.

(A.M.N.H. i. p. 28.)

Type of Sub-Genus, D. isomerioides, M. \& P.

287. Dorcasia isomerioides, Melv. \& Pons.

1898 Dorcasia (Tulbaghinia) isomerioides, M.\& P., A.M.N.H. i.p. 28. pl. 8, f. 10. D.F.

Type in British Museum.

Hab. Cape of Good Hope. Winterhoek Mt., Tulbagh (Marloth).

Owing to its considerable divergence from the type, I append the description of a fine specimen in the South African Museum.

Shell depressed-globose, deeply and openly umbilicate, thin, translucent, thickly covered above with rough, curved striæ, which are continued more smoothly beneath. The upper portion moderately glossy, of dark olive-brown horn colour, beautifully marked on the earlier whorls with pale yellow dots and blotches, merging later into irregular, interrupted concentric streaks; the earlier whorls similarly marked on the underside, but the last is paler, of a greener tint, glossy and unmottled. The interior shows plainly the colour and markings of the epidermis. Spire but little produced, apex flattened. Whorls $4 \frac{1}{2}$, rapidly increasing, rounded, with no trace of carination. Aperture ovate, peristome white and glossy, a little thickened and reflexed. The columellar margin, half-way up between the base of the aperture and the umbilicus, shows trace of two small protuberances on its inner side; above these it becomes more widely reflexed, and forms a sharp angle of about 95 degrees, projecting 
over, but in no way concealing the umbilicus, before rejoining the base of the shell.

Diam. max. 28, min. 21.5 ; alt. $16 \mathrm{~mm}$. Apert. $16 \times 11 \mathrm{~mm}$. Distance between angle of columellar margin and its junction with base of shell, $2.5 \mathrm{~mm}$.

\section{Genus TRIGONEPHRUS, Pilsbry, 1905.}

(Proc. Mal. Soc. vi. p. 286.)

Type of Genus, T. globulus (Müll.).

288. Trigonephrus coagulum (von Martens).

1889 Helix coagulum, von Mts., Sitz.-Ber.Ges.Nat.Fr. Berlin, p.160. D. 1894

1897

$\begin{array}{lll}", \quad & \quad \text { Conch. Mitth. iii, 3. p. 3. D. } \\ , \quad & \quad \text { Archiv. f. Naturg. lxiii, 1. p. 37. pl. 7, }\end{array}$

Type in Zool. Mus. Berlin.

f. 11-14. D.F.

Hab. Great Namaland. On the road from Aos to the Orange River, and near the Lower Orange River (Schenck).

289. Trigonephrus globulus (Müller).

1774 Helix globulus, Müll., Verm. ii.p. 68. D.

$1786 \quad$ " $\quad$, " $\quad$ Chem., Conch. Cab. ix, 2. p. 126. pl. 130, f. 1159,60 . ? D.F.

" $\quad$ " pomatia, var., Chem., Conch. Cab. ix, 2. pl. 130, f.1138. F. 1788 " globulus, Müll., Gmel., Syst. Nat., Ed. 13.i, 6. p. 3629. D.

1817 " " " Dillw., Descr. Cat. ii. p. 923. D.

1822 ", lucana, "Müll.," Lam., Hist. nat. An.s. Vert. vi, 2.p.71. D.

$1838 \quad$ " " " " Desh., Hist. nat. An. s. Vert. viii.p. 37. D.

1811 " globulus, Müll., Pfr., Conch. Cab.p. 33.pl.3,f.11,12. ? D.F.

$1846 \quad " \quad$ " " (cum var, rosacea, Müll.), Pfr., Conch.

Cab. p. xiii. D.N.

1848 " (=lucana, Lam., and rosacвa, Müll.), Krs.,

Südafr. Moll. p. 77. N.

,. Pfr., Mon. Hel. i. p. 319. D.

$1850 \quad$ " $\quad$ " $\quad$. $\quad$, (cum var, rosacea, Müll.), Bs., A.M.N.H. v. p. 217. $N$.

, (=rosacea), Desh., Hist. nat. Moll. i. p. 250. pl. 26, f. 10-12.pl.28,f.11,12. D.F.

$1851 \quad$ " $\quad$ " $\quad$, Rve., Conch. Icon. pl. 41, f. 186. D.F.

$1859 \quad " \quad$ ", " $\quad$ Pfr., Mon. Hel. iv. p. 249. D. 
1879 Helix globulus, Müll., Binu., Ann. N.Y.Acad. Sci.i.p. 361.pl. 14, f. I. $R$.

1880 "globosus " Crvn., P.Z.S. p. 619. (Err.typ.) N.

1884 Dorcasia globulus, Müll., Binn., Ann. N.Y. Acad. Sci. iii. p. 106. pl. 6, f. M. $R$.

1887 Helix (Dorcasia) globulus, Müll., Tryon, Man. of Conch. iii. p. 213. pl. 49 , f. 1. D.F.

1889 „ globulus, Müll., von Mts., Sitz.-Ber. Ges. Nat. Fr. Berlin, p. 160. N.

1895 Dorcasia globulus, Müll., Pilsb., Man. of Conch. ix. pp. 172, 173. pl. 38, f. 8. N.F. pl. 51, f. 3. $R$.

1905 Trigonephrus globulus, Müll., Pilsb., Proc. Mal. Soc. vi. p. 286. pl. 13, f. 6-9. pl. 14, f. 13, 15. A.

1910 Helix (Trigonephrus) globulus, Müll., Bttg., Abh. Senckenb. Naturf. Ges. Frankfurt, xxxii. pp. 440, 441. N.

Original in University Zool. Mus. Copenhagen.

Hab. Cape of Good Hope. Widely distributed along the coastline from Algoa Bay, westward round the Cape Peninsula, and thence northward as far as Port Nolloth. Robben and Dassen Islands.

Craven collected on Robben Island a fully developed sinistral specimen, which is now in the British Museum.

There is a very wide range of variety in this species. Krauss brought into the synonymy $H$. rosacea, Müll, and many later authorities have followed his example; in fact, until more is known of the animals, it is not possible to decide whether all species of Trigonephrus, hitherto described, are not merely forms of globulus.

Much confusion existed among the early authors with regard to it. Chemnitz (1786) figured H. semirugata, Beck, from Ceylon as globulus, while Lamarck (1822) described semimgata as globulus and globulus as lucana. H. globulus, Lam., of Deshayes' earlier writings is also semirugata, and his lucana, globulus, Müll., an error corrected in Hist. nat. Moll., 1850.

290. Trigonephrus gypsinus (Melv. \& Pons.). [S.A.M.] 1891 Helix (Dorcasia) gypsina, M. \& P., A.M.N.H. viii. p. 238. D. 1892 ix. p. $94 . \mathrm{pl} .4$, 1893 ,

,

Type in British Museum. f. $10, F$.

Pilsb., Man. of Conch. viii. p. 262. pl. 28, f. 2 . D.F. 
Hab. Litrte Namaland. Springbok (Lightfoot; Day). Henkries (Lightfoot).

291. Trigonephrus namaquensis (Melv. \& Pons.). [S.A.M.] 1891 Helix (Dorcasia) namaquensis, M. \& P., A.M.N.H. viii. p. 237. D. 1892

$$
\text { , }
$$

1893
" ix. p. 94. pl. 4 , f. $12 . F$. Pilsb., Man. of Conch.viii p. 262.pl. 28,f. 1. D.F.

1910 Helix (Trigonephrus) namaquensis, M. \& P., Bttg., Abh. Senckenb. Naturf. Ges. Frankfurt, xxxii. p. 440. N. Type in British Museum.

Hab. Littule Namaland (Péringuey). Muishond; Meskiep; Kamaggas (Schultze).

292. Trigonephrus porphyrostoma (Melv. \& Pons.). [S.A.M.] 1891 Helix (Dorcasia) porphyrostoma, M.\&P., A.M.N.H.viii.p. 238. D. 1892

$$
\text { " } \quad \text { ", ix. p. 94.pl. 4, }
$$
f. $11 . F$. 1893 ,

Pilsb.,Man.ofConch.viii. p. 262.pl.28,f.3. D.F.

1910 Helix (Trigonephrus) rosacea, Müll., var. porphyrostoma, M. \& P., Bttg., Abh. Senckenb. Naturf. Ges. Frankfurt, xxxii, p. 441. pl. 28, f. 6, 7. N.F.

Type in British Museum.

Hab. Little Namaland (Miss Morris). Port Nolloth (Day).

Great Namaland. Lower Orange River; Gous (Schenck). Angra Pequena (Schneider).

This coarse form of Trigoneptrus is that most frequently found subfossil in old middens. Böttger is probably right in considering it to be a variety of rosaceus, but as the latter is so nearly allied to globulus, it appears advisable to await fuller knowledge of the animal before bringing either into synonymy.

293. Trigonephrus rosaceus (Müller).

[S.A.M.]

1774 Helix rosacea, Müll., Verm. ii. p. 76. D.

$1788 \quad$ " $\quad$ "Gmel., Syst. Nat., Ed.13. i, 6. p. 3636. D.

$1817 \quad$ " " Dillw., Descr. Cat. p. 921. D.

$1838 \quad$ " $\quad$ " Desh., Hist. nat. An. s. Vert. viii. p. 94. N.

1839 " "Jonas, Archiv. f. Naturg. v, 1. p. 339. pl. 9 , f. 3, 4. D.F. 
1841 Helix rosacca, Müll., Pfr., Conch. Cab. p.40. pl. 4, f. 5, 6. pl. 55 (1849), f. 7, 8. D.F.

$1859 \quad$ " $\quad$ "Pfr., Mon. Hel. iv. p. 248. D.

1887 Helix (Dorcasia) rosacea, Müll., Tryon, Man. of Conch. iii. p. 213. pl. 49, f. 100 . D.F.

1910 „, (Trigonephrus) rosacea, Müll., Bttg., Abh. Senckenb.

Naturf. Ges. Frankfurt, xxxii. p. 440. pl. 28, f. 4. D.F.

Specimen ex coll. Müller in University Zool. Mus. Copenhagen.

Hab. CAPE OF Goon Hope. Widely distributed along the western seaboard from the south as far as Namaland, often in company with, and apparently merging into, T. globulus, Müll., of which it is probably a variety.

FAMily HELICID Æ, Gray, 1824.

(Ann. of Philos. viii. p. 107.)

Genus EULOTA, Hartmann, 1842.

(Erd-u. Sussw.-Gast. Schweiz, p. 179.)

Type of Genus, E. fruticum (Müll.).

294. Eulota similaris (Férussac).

1821 Helix similaris, Fér., Tabl. Syst. Moll. pt. 3. p. 47 (or 43). L. , addita, Fér., Tabl. Syst. Moll. pt. 3. p. 71 (or 67).

1834 " woodiana, Lea, Obs. G. Unio, i. p. 169. pl. 19, f, 69. D.F.

1835 ", translucens, King, Zool. Journ. v. p. 339. D.

1836 ", cestus, Bs., Journ. As. Soc. Bengal, v. p. $353 . \quad D$.

$1848 \quad$ " , " A.M.N.H. ii. pp. 160, 161. D.N.

" $\quad$ " similaris, Fér., Pfr., Mon. Hel. i. p. $336 . \quad D$.

$1849 \quad " \quad$ " " (=squalida, Zglr.), Mouss., Moll. von Java, p. 21. pl. 2, f. 4, 5. D.F.

1850 " , , " Pfr., Conch. Cab. p. 341. pl. 60 (1849), f. $13-16 . \quad D \cdot F$.

" Desh., Hist. nat. Moll. i. p. 171. pl. 25в, f. $1-4$. pl. 27 A, f. $1-5$. D.F.

1851 " , " $\quad$ " Rve., Conch. Icon. pl. 34, f. 149. D.F., and pl. 127 (1852), f. $767 . \quad F$.

brardiana, Pfr., P.Z.S. p. $253 . \quad D$.

1852 " , " , Rve., Conch. Icon. pl. 108, f. 604. D.F.

" ", cestus, Bs., Rve., Conch. Icon. pl. 125, f. 751. D.F.

1853 " brardiana, Pfr., Mon. Hel. iii. p. $228 . \quad D$.

$1854 \quad$ " $\quad$ " Conch.Cab.p.385.pl.142(1852),f.7,8. D.F. 
1854 Helix brardiana, Pfr., A.M.N.H. xiii.p. 142. D.

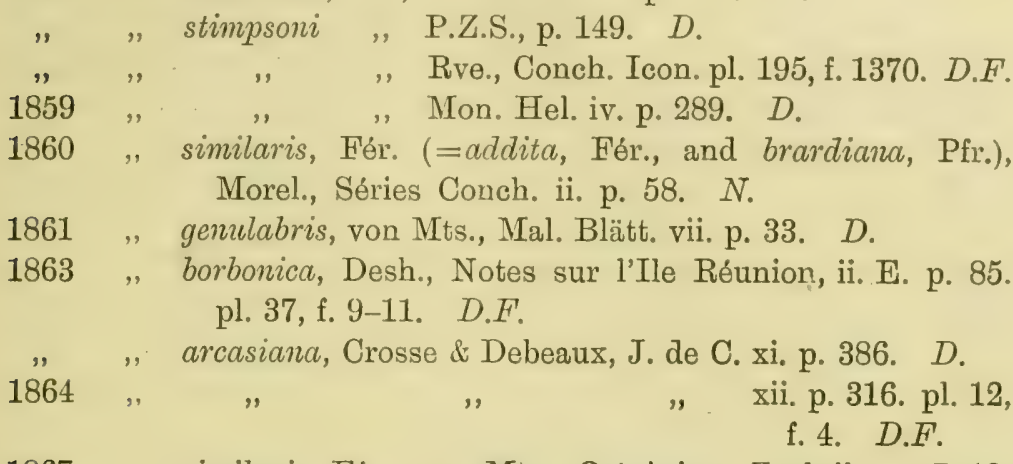

1867 "similaris, Fér., von Mts., Ost-Asien, Zool. ii. pp. 7, 19, 43, 76, 270. D.N.

1868 " " " Cox, Mon. Austral. Landshells, p. 58. pl. 9, f. 14. D.F.

" $\quad$ " borbonica, Desh., Pfr., Mon. Hel. v. p. 504. D.

1874 " (Rhagada) similaris, Fér., Jick., Fauna N.-O.-Afr. p. 70. D.

1875 " similaris, Fér., Morel., Séries Conch. iv. p. 251. N.

1887 ", (Dorcasia) similaris, Fér. (=epixantha, Pfr., Conch. Icon.

f. 454, and squalida, Zglr.), Tryon, Man. of Conch.

iii. p. 205. pl. 46, f. 27-30. pl. 47, f. 33-37. D.F.

1895 Eulota similaris, Fér., Pilsb., Man. of Conch. ix. p. 203. pl. 55, f. $19 . \quad$ N.F. pl. 65 , f. 3, 4. pl. 66, f. $20 . A$.

1905 Helix (Eulota) similaris, Fér., Dautz. \& Fischer, J. de C. liii.

Type- $u b i$ ?

p. 95. $N$. and Synonymy.

Hab. Natal. Durban (Plant; Quekett).

A species of almost world-wide distribution.

Genus COCHLicella, Férussac, 1821.

(Tabl. Syst. Moll. pt. 3. pp. 28 (or 24), 56 (or 52).)

Type of Genus, H. conoidea (Drap.).

295. Cochlicella acuta (Müller).

[S.A.M.]

1774 Helix acuta, Müll., Verm. ii. p. 100. D.

1801 Bulimus ventricosus, Drap. (=H. acuta, Müll.), Drap., Tabl. Moll. Fr. p. $68 . \quad D$.

$1805 \quad$ " $\quad$ Hist. Moll. Fr.p.78.pl.4,f.31,32. D.F.

1821 Helix (Cochlicella) ventrosa, Fér. (=ventricosus, Drap., and acuta, Müll.), Fér., Tabl. Syst. Moll.pt.3,pp.56 (or52),74 (or70). N. 
1822 Butimus ventricosus, Drap., Lam., Hist. nat. An. s. Vert. vi, 2. p. $125 . D$.

1826 Cochlicella ventrosa, Fér., Risso, Hist. nat. Eur. mérid.iv.p.77. D. 1838 Bulimus ventricosus, Drap., Desh., Hist. nat. An. s. Vert. viii. p. 235. $D$.

1841

Küst., Conch. Cab. p. 30. pl. 12, f. 10-12. D.F.

1848 ", ventrosus, Fér., Pfr., Mon. Hel. ii. p. 215. D.

1849 " " " " (=variabilis, Hartm.), Rve., Conch. Icon. pl. 69, f. 499 . D.F.

1855 Helix (Cochlicella) bulimoides, Moq.-Tand., Hist. Nat. Moll. Fr. ii. p. 277. pl. 20, f. 21-26. D.F.A.

1864 " barbara, Bgt., Mal. Algérie, i. p. 286. pl. 32, f. 36-41. D.F.

1874 " (Cochlicella) ventricosa, Drap., Jick., Fauna N.-O.-Afr. p. $96 . D$.

1883 " acuta, Müll. (=B. ventricosus, Drap.), Fagot, Glanages Malac. iii. p. 29. D.N.

$1889 \quad$ " " " Westerl., Fauna Paläarct. Reg.ii, 1.p.366. D. 1894 ". ventricosa, Drap., Coutagne, Mém. Soc. Agr. Sci. et Ind. Lyon, ii. p. $454 . \quad N$.

1895 Bulimus ventricosus, Drap., Locard, Ipsa Draparnaudi Conchylia, p. $100 . \quad N$.

1908 Helix (Cochlicella) acuta, Müll., Germain, Voy. Zool. Khroumirie, p. $233 . \quad N$.

Originals of ventricosa in Naturh. Hofmus. Vienna; acuta-ubi?

Hab. Cape Peninsula. St. James (Connolly).

A South European species, probably of recent introduction. Also recorded from North Africa, Cape Verde Islands, and West Indies.

Genus PUPISOMA, Stoliczka, 1873.

(Journ. Asiatic Soc. Bengal, xiii, 2. p. 32.)

Type of Genus, P. lignicola, Stol.

296. Pupisoma japonicum, Pilsbry.

1902 Pupisoma japonicum, Pilsb., Nautilus, xvi.p. 21. D.

1909

" Hirase, Conch. Mag. iii, 4. pl. 9, f. $32,33, F$.

1912

Bnp., Proc. Mal. Soc. x, p. 46. $N$.

Type in Academy of Natural Science, Philadelphia.

Hab. NataL. Pietermaritzburg;. Ntimbankulu; Edendale; Karkloof; Game Pass (Burnup).

Described from Japan. 
The subjoined figure shows Trach. microscopica (Krs.), which may very possibly belong to the present Genus, in the centre, with the larger orcula on the left and japonicum on the right. $\left(\times 4 \frac{2}{3}.\right)$
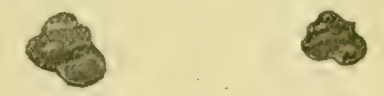

297. Pupisona orcula (Benson).

[S.A.M.]

1850 Helix orcula, Bs., A.M.N.H. vi. p. 251. D.

$1853 \quad$ " $\quad$ "Pfr., Conch. Cab.p. 357.pl. 136 (1852), f. 18-

20. D.F.

". " " $\quad$ " Mon. Hel. iii. p. 42. D.

1855 Nanina orcula, Bs., Gray, Cat. Pulm. p. 75. D.

1886 Pupisona orcula, Bs., Tryon, Man. of Conch. ii. p. 177. pl. 53,

f. $67 . D \cdot F$.

1909

1912

Co-types in British Museum.

" Hirase, Conch. Mag.iii, 4.pl. 9, f.30,31. F. ", Bnp., Proc. Mal. Soc. x. p. 45. N.

Hab. CaPe of Good Hope. Port Elizabeth (Crawford). Grahamstown (Farquhar).

Natal. Pietermaritzburg; Ntimbankulu; Dargle; Edendale; Game Pass (Burnup). Richmond (Wakefield; Cooper). Karkloof (Taynton).

Transvaal. Pretoria (Connolly).

RHoDEsIa. Victoria Falls (Warren).

Originally described from India.

Genus Valilonia, Risso, 1826.

(Hist. nat. Eur. mérid. iv. p. 101.)

Type of Genus, V. rosalia, Risso (pulchella, Müll.).

298. Valtonia excentrica, Sterki.

[S.A.M.]

1850 Helix pulchella, Müll., Bs., A.M.N.H. v. p. 217. L.

1864 Vallonia mimuta, Say, Morse, Journ. Portland Soc. Nat. Hist. i, 1. p. 21 (pars.).

1893

excentrica, Sterki, Proc. Acad. Nat. Sci. Phila. pp. 252, 278. pl. 8, f. B, M. D.A.

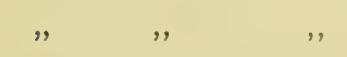

1904

Pilsb., Man. of Conch. viii. p. 249. pl. 32, f. 6-9. D.R.F.

B. B. Woodw., Journ. of Conch. xi. p. 82. $D$. 
Type in coll. Sterki.

Hab. Cape of Good Hope. High Constantia (Benson, 1846). Cape Town (Layard). Wynberg (Lightfoot). Somerset East (Miss Bowker). King Williamstown (Miss Ross). Grabamstown (Farquhar). Port Elizabeth (Crawford).

NATAL。 Pietermaritzburg (Burnup).

Transvaal. Pretoria (McBean).

Very widely distributed over most parts of the globe.

Mr. B. B. Woodward has identified specimens from Wynberg, Pretoria, Grahamstown, Port Elizabeth, and Pietermaritzburg as excentrica, and it is probable that the other occurrences of Vallonia in South Africa refer to the same species. Of course, many early reports of pulchella, such as Benson's in 1850, were made long before excentrica was differentiated.

Genus HELIX, Linné, 1758.

(Syst. Nat., Ed. 10. i. pp. 645, 768.)

Type of Genus, H. pomatia, Lin.

Sub-Genus CRYPTOMPHALUS, Agassiz, 1837.

(Nouv. Mém. Soc. Helv. Sci. Nat. i, 2. p. 5.)

Type of Sub-Genus, H. aspersa, Müll.

299. Heuix aspersa, Müller.

1774 Helix aspersa, Müll., Verm. ii. p. 59. D.

1777 " hortensis, Pennant, Brit. Zool. iv. p. 136. pl.84,f.129. D.F. 1778 Cochlea vulgaris, Da Costa, Test. Brit. p. 72. pl. 4, f. 1. D.F.

1786 Helix aspersa, Müll., Chem., Conch. Cab. ix, 2. p. 125. pl. 130, f. 1156-58. D.F.

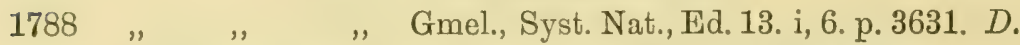
" " grisea, Gmel., Syst. Nat., Ed. 13. i, 6, p. 3649. D.

1797 " restitutoris, Humph., Mus. Calonn. p. 60. D.

1803 ", aspersa, Gmel., Mont., Test. Brit. ii. p. 407. D.

1805 " " Müll., Drap., Hist. Moll. Fr.p. 89. pl. 5, f. 23. D.F. 1807 „ hortensis, Penn., Mat. \& Rack., Linn. Trans. viii. p. 208. D. 1815 " aspersa, Brard, Hist. Coq. Paris, p. 7. pl. 1, f. 1. D.F. 1826 " " Lin., Risso, Hist. nat. Eur. mérid. iv. p. 63. D. $1828 \quad$ " " Lam., Stark, Elem. Nat. Hist. ii. p. 58. D. 1837 " (Pomatia) adspersa, Beck, Index Moll. p. 44.

1840 " (Acavus) aspersa, Müll., Gray, Turton's Man. p. 128. pl. 4, f. 35. D.F. 
Reference List of South African Non-marine Mollusca. 161

1841 Helix aspersa, Müll., Pfr., Conch. Cab. p. 34. pl. 3, f. 6-10. D.F. 1846

1848 " " " , " $\quad$ p. xi. D.

1848. " " , " , Mon. Hel. i. p. 241. D.

$1850 \quad$ " " , Desh., Hist. Nat. Moll. i. p. 269. pl. 18, f. 1-13. pl. 19, f. 1-9. pl. 21в, f. 6, 7. pl. 24, f. 3. pl. 24a, f. 1-7. D.F.

1855 , , , Moq.-Tand., Hist. Nat. Moll. Fr. ii. p. 174. pl. 13, f. 14-32. D.F.A.

1861 " spumosa, Lowe, A.M.N.H. vii. p. $111 . \quad D$.

1879 „, aspersa, Müll., Layard, The Field, Jan. 11th. N.

1883 " (Ponatia) aspersa, Müll., Tayl., Journ. of Conch. iv. p. 89. D.N.A.

1898 Pomatia aspersa, Müll., Stur., S.A. Moll. p. 52. N.

1903 Helix (Helicogena) aspersa, Müll., Kob., Conch. Cab. p. 96. pl. 319, f. 14-20. pl. 320, f. 1-12. D.F.

1910 ", (Cryptomphahus) aspersa, Müll., Tayl., Mon. Brit. Moll. p. 236. pl. 23 (1911). D.F'.A.R.

" (Helicogena) aspersa, Müll., Bttg., Abh. Senckenb. Naturf. Ges. Frankfurt, xxxii. p. $456 . N$.

Specimen ex coll. Müller in University Zool. Mus. Copenhagen.

Hab. CAPE of Good Hope. Port Elizabeth (in coll. Ponsonby).

Cape Peninsula. Very common. Robben Island (in coll. Ponsonby).

Several sinistral specimens have been found at Cape Town (Lightfoot).

This species, said to have been introduced into Cape Town by Mons. Dastre, as a table delicacy, about 1870, has now become one of the greatest pests in gardens throughout the Cape Peninsula. It appears also to thrive on the peculiar local type of wild herbage, and thus threatens to oust many of the rarer, indigenous species from their last foothold, by eating them out of house and home.

Sub-Genus OTALA, Schumacher, 1817.

(Essai d'un Nouveau Système des . . . Vers. p. 191.)

(=Archelix, Albers, 1850.)

Type of Sub-Genus, H. atomaria, Schum. (lactea, Müll.)

300. Helix lactea, Müller.

1774 Helix lactea, Müll., Verm. ii. p. 19. D.

$1786 \quad$ " $\quad$ Chem., Conch. Cab. ix, 2. p. 127. pl. 130, f. 1161 . D.F. 
1822 Helix lactea, Müll., Lam., Hist. nat. An. s. Vert. vi, 2. p. 75. D. 1848 1850 " Pfr., Mon. Hel. i. p. $272 . \quad D$.

\begin{tabular}{|c|c|c|c|}
\hline 186 & $"$ & & Bgt., Mal. Algérie,i.p.122.pl.11,f.1-9. D.1 \\
\hline 188 & $"$ & & $\begin{array}{l}\text { Tryon, Man. of Conch. iv. p. 130. pl. } \\
\quad \text { f. 89-97. D.F. }\end{array}$ \\
\hline
\end{tabular}

Type- $u b i ?$

Hab. Cape of Good Hope. Kowie (Barber, in coll. Layard).

A well-known Mediterranean species, whose sole claim to inclusion in South African lists rests on a fine adult specimen, which was taken alive, together with a smaller, empty shell, in a garden on the bank of the Kowie River in 1897.

Tryon (1888) places in the synonymy irrorata, Say ; punctatissima, Jeniss; flattersiana, Ancey; tagina, Servain; axia, Bgt. ; jacquemenbana, Bgt.; and ? hispanica, Mich.

Sub-Genus EUPARYPHA, Hartmann, 1842.

(Erd-und-Sussw.-Gast. Schweiz, p. 204.)

Type of Sub-Genus, H. rhodostoma, Drap. (pisana, Müll.).

$$
\text { 301. Helix pisana, Müller. }
$$

1774 Helix pisana, Müll., Verm. ii. p. 60. D.

1777 " zonaria, Pennant, Brit. Zool. iv. p. 137. pl.85, f. 133. D.F.

1792 " petholata, Olivier, Zool. Adriat. p. 178. D.

1801 " rhodostoma, Drap., Tabl. Moll. Fr. p. 74 . D.

1803 ", cingencla, Mont., Test. Brit. ii. p. 418, \& Suppl. (1808), pl. 24, f. 4. D.F.

1805 " rhodostoma, Drap., Hist. Moll. Fr.p.86. pl.5, f.13-15. D.F.

1807 " cingenda, Penn., Turton, Brit. Fauna, p. 188. D. -

1827 " " Mont., Brown, Illustr. Conch. Gt. Brit. pl. 40, f. $27,28,33,35,58$. $F$.

1840 " pisana, Müll., Gray, Turton's Man. p. 158. D.

$1848 \quad$ " , " , Pfr., Mon. Hel. i. p. 152. D.

" $\quad$, $\quad$, , Conch. Cab. p. 161. pl. 37, f. 1-12.

pl. 22 (1843), f. 1-6. D.F.

1855 , , , Moq.-Tand., Hist. Nat. Moll. Fr. ii. p. 259. pl. 19, f. 9-20. D.F.A.

$1864 \quad$ " , ", Bgt., Mal.Algérie,i.p.234.pl.26,f.1-10. D.F. 1874 " (Euparypha) pisana, Müll., Jick., Fauna N.-O.-Afr. p. 85. D.N. 
Reference List of South African Non-marine Mollusca. 163

1895 Helix (Euparypha) pisana, Müll., Pilsb., Man. of Conch. ix. p. 335 . pl. 43 , f. 37,38 . pl. 61 , f. 1-5. D.F.A. 1902 Xerophila pisana, Müll., Swanton, Tourn. of Conch. x. p. 194. N. 1910 Helix (Euparypha) pisana, Müll., Bttg., Abh. Senckenb. Naturf. Ges. Frankfurt, xxxii. p. $456 . \quad N$. 1911, 12 „, Müll., Tayl., Mon. Brit. Moll. p. 368 pl.30,f.1-21.pl.31,f.1-21.D.F.A.R

Specimen ex coll. Müller in University Zool. Mus. Copenhagen.

Hab. Cape of Good Hope. Cape Peninsula (very common). .Stellenbosch (Miss Lightfoot). Somerset West; Gordon's Bay (Connolly). Port Elizabeth (Crawford). Robben Island (in coll. Ponsonby).

Natar. Durban (Longstaff).

The earliest recorded appearance of $H$. pisana in South Africa was in 1881, when Mr. W. G. Fairbridge took three specimens on the now demolished Gallows Hill, near Cape Town Docks. Since that date it has spread enormously along the seaboard of the Peninsula, and its arrival at Stellenbosch appears to denote that it is extending its travels inland.

Taylor (1911) includes in its synonymy strigata, Dillw., 1817; maculata, Mke., 1828 ; catocyphia, Bgt., 1860 ; pisanopsis and hyperplate, Bgt., 1880 ; agaroi, carpiensis, djerbanica, gergisensis, hamadanica, salemensis, monroi, zitanensis, Letourneux and Bgt., 1887 ; chambardi, Let., 1887 ; couturieri, cuttati, lenoleuca, subpisana, and thinophila, Bgt., 1887 ; donatii and levesquei, Berthier, 1887 ; byrsa and radesiana, Marés, 1887 ; dermoi, pisanella, and olivaresi, Servain, 1887 ; barbozana, bocagei, and machadoi, Locard, 1899 ; while immature examples appear to have been named astivalis, Bgt.; cince, Klett; and leucostoma, Risso.

Family ENID无, B. B. Woodward, 1903.

(Journ. of Conch. x. pp. 354, 358.)

(=Buliminidæ, Auctt.)

Genus ENA, Leach (Mss. 1820), 1831.

(Turton's Manual, 1831, p. 80, and 1840, p. 181.

(=Bulimina, Ehrenberg, 1831 (Butiminus, Auctt.), nec d'Orbigny, in Foraminifera, 1826.)

Type of Genus, E. montance (Drap.).

The South African Enida can be divided, on form alone, into certain well-marked groups, in the following manner:- 
(i) Pachnodus, Albers, 1860 (Die Helic. p. 230, Type, in error, velutina, Pfr.; emend., Bgt., 1889, Moll. de l'Afr. équat. p. 64, Type spadicea, Mke.).

Bourguignat's rejection of velutina as type of Pachnodus seems perfectly sound. If spadicea is not acceptable, a new name is necessary for the group, which comprises-

arenicola, Bs. ; carinifera, M. \& P.; drakensbergensis, Smith; mcbeaniana, Bnp.; natalensis, Krs. and spadicea, Mke. (=vitellina, Pfr.).

(ii) Comulinus, von Martens, 1895 (Nachr. d. Deutsch. Mal. Ges. p. 180, Type, comulus, Rve.).

comulus, Rve.; metuloides, Smith; transvaalensis, M. \& P., and probably burmupi, dimera, and maritzburgensis, M. \& P., and meridionalis, Pfr.

(iii) Xerocerastus, Kob. \& Mlldff., 1902 (Conch. Cab. p. 1021, Type, damarensis, Ad.).

(=Eburnea, Mousson, 1887 (J. de C. p. 295), nec Eburna, Lam., 1801, nec Ebumea, Flem., 1828).

burchelli, Gray ; damarensis, Ad. ; hottentota, Gray (= pygmcea, Ad.); layardi, M. \& P.; opposita, Mouss.; psammophila, schultzei, and subteres, Bttg., and zuluensis, M. \& P.

(iv) Rhachisellus, Bourguignat, 1889 (Moll. de l'Afr. équat. p. 68, as Rachisellus. Type punctata, Anton.)

Thiele (Deutsch. Zent.-afr.-Exp., 1911, p. 201) shows that punctata is not acceptable as type of Rhachis, Albers, but is rightly placed in Rhachisellus. Although possibly not belonging to the same Subgenus, the following shells fall, roughly, into the same group:-

dubiosa, Stur. ; melanacme, mozambicensis, and petersi, Pfr.; punctata, Ant. (=jejuna, M. \& P.); spilogramma and sticta, von Mts.

Thiele (l.c.) has, on account of radular peculiarities, proposed to include melanacme and mozambicensis in a new Sub-genus Rhachidina, the type of which is the West African tumefacta (Rve.).

(v) Ena pentheri (Stur.).

(vi) Group of $E$. bowkeri (Sow.). bowkeri, Sow., and muptialis, M. \& P.

(vii) Ena boivini (Morel.) (=movenensis, Stur.).

In the following pages the species are arranged in alphabetical order.

Noтe.-Eulimoides, Gray, and namibicus, Bttg., were described as Enida, but apparently belong to Zootecus, Westerl., a Genus of Stenogyrina. 
Reference List of South African Non-marine Mollusca. 165

302. Ena (Pachnodus) arenicolail(Benson). [S.A.MI].

1856 Bulimus arenicola, Bs., A.M.N.H. xviii. p. 433. D.

1859

" Pfr., Mon. Hel. iv. p. $481 . \quad D$.

1901 Buliminus (Pachnodes) arenicola, Bs., Kob., Conch. Cab. p. 757. pl. 111, f. 5, 6. D.F.

Specimen ex auct. in University Museum of Zoology, Cambridge.

Hab. CaPe of Good Hope. Caffraria, near Waterloo Bay; near Mossel Bay (Layard).

Natal. Durban; Pietermaritzburg; Lower Umkomaas; Port Shepstone; Tongaat (Burnup).

Zululand. Dukuduku (Toppin).

303. Ena borvini (Morelet).

1860 Glandina boivini, Morel., Séries Conch. ii. p. 72. pl. 5, f. 5. D.F. 1887 Bulimus „ „ Grandidier, Bull. Soc. Mal. Fr. iv. p. 187. N.

1890 " (Cerastus) mamboiensis, Smith, A.M.N.H. vi. p. 153. pl. 5, f. 7. D.F.

1897 Buliminus boivini, Morel., von Mts., D.-O.-Afr. p. 61. N.

1898 " movenensis, Stur., S.A. Moll.p.66.pl.2, f.44-51. D.F.

1899 " boivini, Morel. (=mamboiensis), Smith, P.Z.S. p. 587. N.

$1900 \quad$ " (Cerastus) boivini, Morel., Kob., Conch. Cab. p. 635. pl. 97, f. 2. D.F.

(Pachnodes) movenensis, Stur., Kob., Conch. Cab. p. 632. pl. 96, f. 19-21. D.F.

Type of boivini in British Museum; movenensis in Naturh. Hofmus. Vienna.

Hab. Eastern Zululand. Elscheleselwanhla (Toppin).

Lorenzo Marques. Delagoa Bay (Connolly). Movene (movenensis, Penther).

Also from several localities in East Africa, the type of boivini coming from Mombasa.

There seems no reason for separating movenensis, Stur., from the present species. Until connecting links are known, E. ptychaxis, Smith (A.M.N.H. vi. 1890, p. 147), which von Martens has placed in the synonymy, appears separable by its more elongate shell and very distinct columellar fold, which is only just traceable in boivini.

304. ENa botwkeri (Sowerby).

1889 Buliminus (Mesembrinus ?) bowkeri, Sow., P.Z.S. p. 581. pl. 56, f. 5. D.F. 
1900 Butiminus (Pachnodes) boukeri, Sow., Kob., Conch. Cab. p. 657. pl. 101, f. 2. D.F.

Originals in British Museum, type not specified.

Hab. Cape of Good Hope. Glen Avon Falls, Somerset East (Miss Bowker).

305. Ena (Xerocerastus) burchelli (Gray). [S.A.M.] 1834 Bulimus burchellii, Gray, P.Z.S. p. 66. D.

1848 " " , " Pfr., Mon. Hel. ii. p. 162. D.

$1849 \quad$ " , " Rve., Conch. Icon. pl. 76, f.548. D.F. 1902 ", (Xerocerastus?) burchellii, Gray, Kob., Conch. Cab. p. 962. D.

Type in British Museum.

Hab. British Bechuanaland. Near Lattakoo (Takun), (fide Gray). Kuruman (Moffatt).

Bechuanaland. Kalahari (Penderill-Longlands).

Cape of Good Hope. Prieska (Gibbons). Douglas (Miss Orpen).

Griqualand West. Blaauwbosch Poort, Hay District (Day). Griquatown (Barrett Hamilton). Griquatown (Gibbons). Riverton (Miss Wilman).

The loc. "Natal," given by Sturany is improbable.

306. Ena (Conulinus) Burnupi (Melv. \& Pons.).

1903 Butiminus (Pachnodus) bumupi, M. \& P., A.M.N.H. xii.p. 605. pl. 31 , f. 5. D.F.

Type in British Museum.

Hab. Zululand. Lower Umfolosi Drift (Burnup). Makowe (Crosly).

307. Ena (Pachinodus) carinifera (Melv. \& Pons.). [S.A.M.] 1897 Butiminus (Pachnodus) carinifer, M. \& P., A.M.N.H. xix. p. 637. pl. 17, f. 8. D.F.

1901 " (Pachnodes) carinifer, M. \& P., Kob., Conch. Cab. p. 762. pl. 111, f. 21. D.F.

Type in British Mnseum.

Hab. Natal. Gordon Falls; Pietermaritzburg (Burnup).

Cape of Good Hope. Knysna (Purcell). Grahamstown; Bedford; Port Elizabeth (Farquhar). Pirie (Miss Ross).

Although perfectly distinct, this species is at times very closely 
Reference List of South African Non-marine Mollusca. 167

approached by $E$. natalensis (Krs.), and it may possibly happen that localities given for it really refer to the latter.

In carinifera the keel, especially in mature shells, is always more pronounced, and the whorls, convex below the suture, become concave both immediately above and beneath the keel.

A pretty milk-white variety is found at Gordon Falls.

308. Ena (Conulinus) conulus (Reeve).

1849 Bulimus comulus, Rve., Conch. Icon. pl. 78, f. 577. D.F.

1853 .. " " Pfr., Mon. Hel. iii. p. 440. D.

1902 Buliminus (Conulinus) comulus, Rve., Kob., Conch. Cab. p. 952. pl. 133 , f. 8 . D.F.

Type in British Museum.

Hab. Natal. "Port Natal" (Mus, Cuming).

Lorenzo Marques. Rikatla (Junod).

Zululand. Entendweni (var.; Toppin).

309. Ena (Xerocerastus) damarensis, H. Adams.

1870 Bulimus damarensis, H. Ad., P.Z.S. p. 9. pl. 1, f. 17. D.F.

" " dammarensis, Pfr., Mal. Blätt. xvii. p. $93 . \quad D$.

" " " $\quad$ " Novit. Conch.iv.p. 3. pl. 109, f. 7, 8.

1877 „, damarensis, Pfr., Mon. Hel. viii. p. 177. D.

1887. Buliminus (Eburnea) damarensis, H. Ad., MLuss., J. de C. xxxv. p. 295. $N$.

1889 ", damarensis, H. Ad., von Mts., Sitz.-Ber. Ges. Nat.

Fr. Berlin, p. $162 . \quad N$.

1900 ", (Zootecus) damarensis, H. Ad., Kob., Conch. Cab. p. 662. pl. 101, f. 17, 18. D.F.

1910 Ena (Eburnea) damarensis, H. Ad., Bttg., Abh. Senckenb.

Naturf. Ges. Frankfurt, xxxii. p. 443. N.

Type in British Museum.

Hab. Damaraland (coll. Adams). Ussab (fide von Martens) and Ubeb, on the Khan River, N. of Tsoachaul (Schenck). Omaruru (Schinz). "Kurikaubmund am Swakop (Kurikop bei Otjikango)" (Rintelen).

Ovampoland. Ovambonde (Chapman, fide Layard). Grootfontein, near Upingtonia, and Epitonna, S.E. of Ondonga (Schinz).

This species was described by H. Adams in January, 1870, and by Pfeiffer in July of the same year; hence the English author has priority. 
var. MrNoR, Pfeiffer.

1870 Bulimus dammarensis, var. minor, Pfr., Mal. Blätt. xvii. p. 94. D. ", , " , N Novit. Conch. iv. p. 4. pl. 109, f. 5, 6. D.F. 1887 Buliminus (Ebumea) damarensis, H. Ad., var. exspectata, Mouss., J. de C. xxxv. p. 295. pl. 12, f. 4. N.F. 1904 " damarensis, (?) var. exspectatus, Mouss., von Mts., Die Kalahari, p. 754. N.

1910 Ena (Eburnea) damarensis, H. Ad., var. minor, Pfr. (=var. exspectata, Mouss.), Bttg., Abh. Senckenb. Naturf. Ges. Frankfurt, xxxii. p. 443.

Type in Stettin Museum.

Hab. Damaraland (fide Pfeiffer). Ubeb on the Khan River (Schenck). Omaruru (Schinz).

Ovampoland. Upingtonia (Schinz).

Bechuanatand. Meno a kwena (about $24^{\circ}$ E. long. and $20^{\circ} \mathrm{S}$. lat.), in marly sandstone (Passarge).

var. subradrata, Böttger.

1910 Ena (Eburnea) damarensis, H. Ad., var. subradiata, Bttg., Abh. Senckenb. Naturf. Ges. Frankfurt, xxxii. p. 443. D.

Type in Senckenberg Mus. Frankfurt.

Hab. Damaraland. "Kurikaubmund am Swakop (Kurikop bei Otjikango)"' (Rintelen).

310. Ena (Conulinus) dimera (Melv. \& Pons.). [S.A.M.] 1901 Buliminus (Rhachis) dimerus, M. \& P., A.M.N.H. viii. p. 320. pl. 2, f. 13. D.F.

Type in British Museum.

Hab. Natal. Karkloof Bush (McBean).

Cape of Good Hope. King Williamstown (Godfrey).

311. Ena (Pachnodus) drakensbergensis (Smith).

1877 Bulimus (Pachnodus) drakensbergensis, Smith, A.M.N.H. xx. p. 538 . $D$.

1879 Buliminus natalensis, Krs., var. draakensburgensis, Smith, Binn., Ann. N.Y. Acad. Sci. i. p. 362. pl. 14, f. J. $R$.

1901 „ (Pachnodes) drakensbergensis, Smith, Kob., Conch.

Type in British Museum. Cab. p. 758. pl. 111, f. 7, 8. D.F. 
Hab. Transvaat. East slope of Drakensberg, near Lydenburg Goldfields (ex coll. Sowerby). Pilgrim's Rest (Craven).

312. Ena (? Rhachiseluus) dubiosa (Sturany).

1898 Buliminus (Rhachis) dubiosus, Stur., S.A. Moll. p. 64.pl. 2, f. 45, 46. D.F.

1900 , Kob., Conch. Cab. p. 632. pl. 96, f. 17, 18. D.F.

Type in Naturh. Hofmus. Vienna.

Hab. Lorenzo Marques. Matolla (Penther).

313. Ena (Xerocerastus) hottentota (Gray).

1838 Bulimus hottentota, Gray, Alexander's Expedition, ii. p. 269. D. 1870 Bulimulus pygmaus, H. Adams, P.Z.S. p. 9. pl. 1, f. 18. D.F. 1877 Pfr., Mon. Hel. viii. p. 123. D. 1910 Ena (Eburnea) pygmaa, H. Adams, Bttg., Abh. Senckenb. Naturf. Ges. Frankfurt, xxxii. p. 442. N.

Types in British Museum.

Hab. Great Namaland. Near Great Fish River (Alexander).

Damaraland (in coll. H. Adams). Flats by the Khan River, N. of Tsoachaul (Schenck). Ussab (fide von Mts.). 140 kilos from Swakopmund (Rintelen).

Ovampoland. Ovambonde (Chapman, fide Layard).

314. Ena (Xerocerastus) liayardi (Melv. \& Pons.).

1892 Buliminus layardi, M. \& P., A.M.N.H. ix. p. 90. pl. 5, f. 11. D.F. 1901

" Kob., Conch. Cab. p. 793. pl. 117, f. 3. D.F.

Type in British Museum.

Hab. Bechuanaland. Kobis, probably just S. of Lake Ngami (in coll. Layard).

315. Ena (Pachnodus) mebeaniana, Burnup. [S.A.M.]
[S.M. 1905 Ena (Pachnodus) mebeaniana, Bnp., Proc. Mal. Soc. vi. p. 302. pl. 16, f. 1, 2. D.F.

Type in British Museum.

Hab. Transvaal. Pretoria District (McBean). Pietpotgietersrust (Connolly).

Bulimus spadiceus, var., quoted by Krauss (Südafr. Moll., 1848, p. 79) from Mt. Mohapaani, is possibly referable to this species. 
316. Ena (Conulinus) maritzburgensis (Melv. \& Pons.). [S.A.M.] 1893 Buliminus (Pachnodus) maritzburgensis, M. \& P., A.M.N.H. xii. p. 105. pl. 3, f. 5 . D.F.

1901 Buliminus (Pachnodes) maritzburgensis, M. \& P., Kob., Conch. Cab. p. 760. pl. 111, f. 16, 17. D.F.

Type in British Museum.

Hab. NATAL. Thornybush, and other localities near Pietermaritzburg (Burnup).

\section{Ena (Rhachidina) melanache (Pfeiffer).}

1855 Bulimus melanacme, Pfr., P.Z.S. p. 96. pl, 31, f. 8. D.F.

$1859 \quad " \quad \quad, \quad$ " Mon. Hel. iv. p. 486. D.

" $\quad$ " (Rhachis) melanacme, Pfr., von Mts., Mal. Blätt. vi. p. 212. pl. 2, f. 8. N.F.

1860 Buliminus (Rhachis) melanaeme, Pfr., von Mts., Die Helic. p. 231. (Err. Typ.)

$1869 \quad$ " $\quad$ " melanacme, Pfr., von Mts., Nachrichtsbl. d. Deutsch. Mal. Ges. p. 152. N.

1889 Pachnodus sesanromum, Ancey in Mss., Bgt., Moll. de l'Afr. équat. p. 66. pl. 3, f. 2, 3. D.F.

1897 Buliminus (Rhachis) melanacme,Pfr.,von Mts.,D.-O.-Afr.p.75. N. 1900 " Kob., Conch. Cab. p. 623. pl. 95, f. 8. D.F.

Type in British Museum.

Hab. Mozambique. Tette (Peters).

Also found in East Africa. Pfeiffer's loc. Tette is of doubtful authenticity. von Martens (1897) remarks: Pfeiffer gives Tette, on the Zambesi, as the finding-place of the specimens found by Peters; but on Peters' labels in the Berlin Museum only Querimba, not Tette, is written, while for $B$. punctatus both localities are vouched for in his handwriting.".

Melvill and Standen (1907) quote this species from Petauke, Northern Rhodesia.

318. Ena (Conulinus) meridionatis (Pfeiffer). [S.A.M.] 1817 Bulimus meridionalis, Pfr., P.Z.S. p. 231. D. 1848 " Mon. Hel. ii. p. $108 . \quad D$

" " " $\quad$ Rve., Conch. Icon.pl. 56,f.370. D.F 1 S98 Buliminus (Rhachis) meritionalis, Pfr., Stur., S.A. Moll.p.64. N. 1901 " (Conulinus) meridionalis, Pfr., Kiob., Conch. Cab. p. 759. pl. 111, f. 14, 15. D.F. 
Type in British Museum.

Hab. "South Africa" (in coll. Cuming, fide Pfeiffer).

CaPe of Good Hope. Port Elizabeth (Crawford; Earquhar).

? Lorenzo Marques. Matolla (Penther, fide Sturany).

\section{Ena (Conulinus) metuloides, Smith.}

1899 Buliminus (Conulinus) metuloides, Smith, P.Z.S. p. 587. pl. 33, f. 43 . D.F.

Type in British Museum.

Hab. Rhodesra. Victoria Falls (Becker).

Described from Zomba, Nyassaland.

The Rhodesian specimens are a little stouter than the type, but do not appear separable.

\section{Ena (Rhachidina) mozambicensis (Pfeiffer).}

1846 Bulimus mozambicensis, Pfr., Symb. iii. p. 85. D.

1848

1849

1859

1869
" Mon. Hel. ii. p. 177. D.

", Rve.,Conch.Icon.pl.58,f.328.D.F.

mozambicensis, Pfr., Mon. Hel. iv. p. 473. D.

(Rhachis) mozambicensis,Pfr, ,von Mts., Nachrichtsbl.d.

Deutsch mal. Ges. i. p. 150. D.

1879 Buliminus mozambicensis, Pfr., Gibbons, Journ. of Conch, ii. p. $144 . \quad N$.

\begin{tabular}{|c|c|c|c|}
\hline & & spekei, $\mathrm{Bg}$ & st., Moll. de l'Égypte, p. 4. D. \\
\hline 1897 & & (Rhachis) & $\begin{array}{l}\text { mossambicensis, Pfr. (cum var. spekei, } \\
\text { Bgt.), von Mts., D.-O.-Afr. p. } 74 . \text { N.D. }\end{array}$ \\
\hline 1899 & ", & ", & $\begin{array}{l}\text { mozambicensis, Pfr., Junod, Bull. Soc. } \\
\text { Vaudoise, xxxv. p. 279. N. }\end{array}$ \\
\hline 1901 & " & ", & $\begin{array}{l}\text { mozambicensis, Pfr., Kob., Conch. Cab. } \\
\text { p. } 748 \text {. pl. 110, f. 7, 8. D.F. }\end{array}$ \\
\hline
\end{tabular}

Type in British Museum.

Hab. Lorenzo Marques. Rikatla (Junod).

Originally described from Mozambique (coll. Cuming).

321. Ena (Pachnodus) natalensis (Krauss). [S.A.M.] 1846 Bulimus natalensis, Krs., Pfr., Symb. iii. p. 86. D.

1848

,"

1849
" Südafr. Moll. p. 78. pl. 5, f. 1. D.F.

" Pfr., Mon. Hel. ii. p. 48. D.

" Rve., Conch. Icon. pl. 62, f. 430. D.F. (pessinza). 
1898 Buliminus (Pachnodus) natalensis, Krs., Stur., S.A. Moll.p.63. N. 1899 " (Pachnodes) , , Kob., Conch.Cab.p.621. pl. 94 , f. 14, 15. D.F.

Type in Stuttgart Museum.

Hab. Natal. Near Natal Bay (Krauss). Drakensberg (Wahlberg). Umkomaas; Pietermaritzburg; Tongaat; Pinetown ; Krantz Kloof (Burnup).

Cape of Good Hope. Port Elizabeth; Springfields (Reeve). Kowie (Farquhar). Port St. John's (Shortridge). Knysna (Layard).

Lorenzo Marques. Rikatla (Junod). Delagoa Bay (smaller var., fide Sturany).

322. Ena nuptialis (Melv. \& Pons.).

1894 Buliminus nuptialis, M. \& P., A.M.N.H. xiv.p.92.pl.1, f.5. D.F. 1901 " (Pachnodes) muptialis, M. \& P., Kob., Conch. Cab. p. 762. pl. 111, f. 22 . D.F.

Type in British Museum.

Hab. Cape of Good Hope. Craigie Burn, Somerset East (Mrs. Barber). Elandsberg Mountain, Cradock (Farquhar).

323. Ena (Xerocerastus) opposita (Mousson).

1887 Helix (Cochlicella) opposita, Mouss., J. de C. xxxv. p. 293. pl. 12, f. 2. D.F.

1900 Buliminus (Zootecus?) oppositus, Mouss., Kob., Conch. Cab. p. 661. pl. 101, f. 13. D.F.

1904 Cochlicella opposita, Mouss., von Mts., Die Kalahari, p. 755. N. 1910 Ena opposita, Mouss., Bttg., Abh. Senckenb. Naturf. Ges. Frankfurt, xxxii. p. 444. N.

Hab. Ovampoland. Upingtonia (subfossil, Schinz).

Bechuanaland. Meno a kwena (subfossil, Passarge).

Described from a single specimen, compared to Cochlicella ventricosa, Drap;, and terveriana, Webb.

324. ENA PENTHeRI (Sturany).

1898 Buliminus (Rhachis) pentheri, Stur., S.A. Moll. p. 65. pl. 2, f. 47, 48. D.F.

1900 " Kob., Conch. Cab. p. 631. pl. 96, f. $15,16$. D.F.

Type in Naturh. Hofmus. Vienna.

Hab. Lorenzo Marques. Matolla (Penther). 
325. Ena (? Rhachisellus) petersi, Pfeiffer.

1855 Bulimus petersi, Pfr., P.Z.S. p. 97. D.

1859

" von Mts., Mal. Blätt. vi. p. 213. N.

" " " Mon. Hel. iv. p. 496. D.

1902 Buliminus (Rhachis) petersi, Pfr., Kob., Conch. Cab. p.986. D. Type in British Museum.

Hab. Lorenzo Marques. Tette (Peters).

326. Ena (Xerocerastus) panamophila (Böttger).

1886 Buliminus (Mastus) psammophilus, Bttg., Ber. Senckenb. Naturf. Ges. Frankfurt, p. 23. pl. 2, f. 2. D.F. $1887 \quad$ " psamophilus, Bttg., Mouss., J. de C. xxxv. p. 295. N. $1900 \quad$ " (Zootecus) psammophilus, Bttg., Kob., Conch. Cab. p. 663. pl. 101, f. 19-21. D.F.

1910 Ena (Eburnea) psammophila, Bttg., Abh. Senckenb. Naturf. Ges. Frankfurt, xxxii. p. 443 . N.

Type in Senckenberg Mus. Frankfurt.

Hab. Bechunaland. Khuis, western border of Kalahari (Nolte).

Great Namaland. Choarib (Hermann).

327. Ena (Rhachisellus) punctiata (Anton). [S.A.M.]
[S.

1839 Bulimus punctatus, Ant., Verz. Conch. Samml. p. 42. D.

1848

1849

1851

" Pfr., Mon. Hel. ii. p. $212 . \quad D$.

,

" Rve., Conch. Icon. pl. 55, f. 452. D.F.

"Desh., Hist. Nat. Moll, ii, 2. p. 86.

1854,55 pl. 157, f. 7, 8. D.F.

", (=solatus, Bs. in Mss.), Pfr., Conch. Cab. p. 229. pl. 62, f. 22-24. D.F.

1859 Bulimus (Rhachis) punctatus, Ant., von Mts., Mal. Blätt. vi. p. $213 . \quad N$.

1869 Bulimimus (Rhachis) punctatus, Ant., von Mts., Nachrichtsbl. d. Deutsch. Mal. Ges. i. p. $153 . N$.

1876 Bulimus punctatus, Ant., Hanley \& Theobald, Conch. Indica,

1879 p. 10. pl. 20, f. 10. $F$.

1889 Rachisellus lectoulxi, "Bibbons, Journ. of Conch. ii. p.144. N. f. 10, 11. D.F.

1893 Buliminus (Pachnodus) jcjunus, M. \& P., A.M.N.H. xii. p. 106. pl. 3, f. 7. D.F.

1897

(Rhachis) punctatus, Ant. (cum varr. ledoubxi, Bgt., and variolosus, Morel.), von Mts.,D.-O.-Afr.p.76. N. 
1901 Buliminus (Pachnodes?) jejunus, M. \& P., Kob., Conch. Cab. p. 794. pl. 117, f. 5. D.F.N.

Types in British Museum.

Hab. Lorenzo Marques. Tette (Peters). Lebombo Mountains (Barber).

Rhodesia. Near Gwelo (Dodds).

Northern Transvaal (jejuna, Bowker).

? Ovampoland. Upingtonia; Epitonna (fide Sturany).

? Damaraland. Omaruru (fide Sturany).

An Indian species imported through commerce, and rather widely distributed in East Africa. Very careful comparison has been made of the type set of jejuna, in the British Museum, with immature specimens of punctata from Daressalam, and no specific difference can be found between them.

Ena (Rhachisellus) férnssaci (Dkr.), which some writers have - included in the synonymy, appears to be a distinct species.

\section{Ena (Xenocerastus) schultzei, Böttger.}

1910 Ena (Eburnea) schultzei, Bttg., Abh. Senckenb. Naturf. Ges. Frankfurt, xxxii. p. 442. pl. 28, f. 9. D.F.

Type in Senckenberg Mus. Frankfurt.

Hab. Bechuanaland. Kooa; Sekuma District: Kang; Kakir and Lekututu Districts (Schultze).

Griqualand West. Near Cypher Krantz (Day).

329. Ena (Pachnodus) spadicea (Menke). [S.A.M.] 1846 Bulimus spadiceus, Mke., Pfr., Symb. iii. p. 87. D.

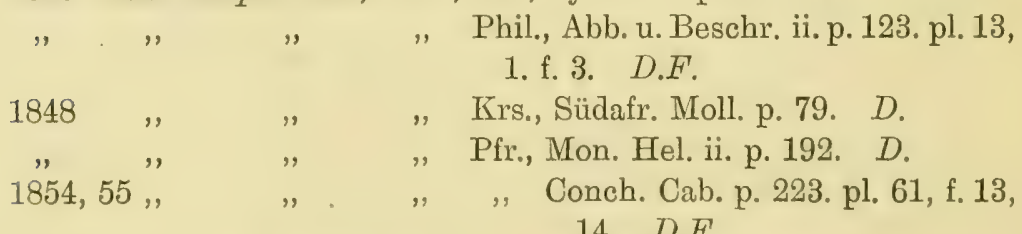

1854 " vitellinus, Pfx., P.Z.S. p. 57. D.

1859 " ", " Mon. Hel. iv. p. 480. D.

1902 Buliminus (Pachnodes) vitellinus, Pfr.,Liob.,Conch.Cab.p.997. D.

Type of spadicea in Stettin Museum; vitellina in British Museum.

Hab. Natat. "In the forests" (Krauss). Umlaas River (fide Sturany). Port Shepstone; Durban (Burnup).

CAPE of Good Hope. Somerset East (Miss Bowker). Kentani (Kolbe). Port Elizabeth (Crarvford). East London (fide Layard). 
Transvaal. Barberton (Cregoe).

Vitellina was described from "Natal." The type is simply an example of spadicea.

Var. MINOR, Pfeiffer.

1848 Bulimus spadiceus, Mke. var., Krs., Südafr. Moll. p. 79. D.

", ", $\quad$, var. minor, Pfr. (Butimus caffer, Krs., in litt.), Pfr., Mon. Hel. ii. p. 192. D.

Krauss' type in Stockholm Museum.

If the above two quotations refer to the same shell, which is not quite certain, the Hab. is Mt. Mohapaani, probably in the Northern Transvaal, and the variety may possibly be identical with E. mebeaniana, Bnp.

330. Ena (? Rhachisellus) spilogramia (von Martens).

1859 Bulimus spilogrammus, von Mts.,Mal.Blätt.vi.p.214.pl.2,f.9. D.F. 1860 ", (Pachnodus) spilogrammus, von Mts., Die Helic.p.230. D. 1868 " spilogrammus, von Mts., Pfr., Mon. Hel. vi. p. 56:- D. 1900 Butiminus (Pachnodus) spilogrammus, von Mts., Kob., Conch. Cab. p. 624. pl. 95, f. 9. D.F.

Type in Zool. Mus. Berlin.

Hab. Lorenzo Marques. Tette (Peters).

331. Ena (? Rhachisellus) sticta (von Martens). [S.A.M.] 1859 Bulimus (Rhachis) stictus, von Mts., Mal. Blätt. vi. p. 211. pl. 2, f. 6. D.F.

$\begin{array}{ll}1860 & , \\ 1868 & , \\ 1899 \text { Buliminus , , } \\ 1900 \quad, \quad,\end{array}$

Type in Zool. Mus. Berlin.

Hab. Lorenzo Marques. Tette (Peters). Manica Land (Selous). RHodesia. Three miles east of Umtali (Dodds).

Zululand. Umbonambi (Toppin). White Umfolosi Flats (Gibson). Also reported from Angoni Land and other localities in Central Africa and Northern Rhodesia.

332. Ena (Xerocerastus) subteres, Böttger.

1910 Ena (Ebumea) subteres, Bttg., Abh. Senckenb. Naturf. Ges. Frankfurt, xxxii. p. 444. pl. 28, f, 8. D.F.

Type in Senckenberg Museum, Frankfurt. 
Hab. Damaraland. 140 kilos inland from Swakopmund(Rintelen.) Founded on a single, dead specimen.

333. Ena (Conulinus) transvaalensis (Melv. \& Pons.). 1893 Butiminus transvaalensis, M. \& P., A.M.N.H. xii. p. 105. pl. 3, f. $6 . D \cdot F$.

1901 Kob., Conch. Cab. p. 794. pl. 117, f. 4. D.F.

Type in British Museum.

Hab. Northern Transvaal (Bowker).

334. Ena (Xerocerastus) zuluensis (Melv. \& Pons.).

1898 Buliminus zuluensis, M.\& P., A.M.N.H.ii.p. 127. pl. 7, f.5. D.F. 1902 " (Xerocerastus) zuluensis, M. \& P., Kob., Conch. Cab. p. 895. pl. 128 , f. 3 . D.F.

Type in British Museum.

Hab. Zululand. Inseyi River (fide M. \& P.).

FAmily VERTIGINID A , B. B. Woodward, 1903.

(Journ. of Conch. $x_{*}$ pp. 354, 360.)

(=Pupidæ, Pupæ, \&c., auctt.)

Genus LEUCOCHILOIDES, Pfeiffer, 1881.

(Nomenclator, p. 292.)

Type of Genus, L. lardeus (Pfr.).

Adequate discussion of this Genus and the species attributed to it would fill far too much space for inclusion in the present work. Pilsbry and Vanatta, in their "Partial Revision of the Pupæ of the United States" (Proc. Acad. Nat. Sci. Phila., 1900, p. 582), considered both Leucochila, Albers, 1860, and Leucochiloides to be dentical with the older Pupoides, Pfr. (Mal. Blätt. i. 1854, p. 192). There is, however, an earlier Pupoides, proposed by Férussac (Tabl. Syst. pt. 3. 1821, p. 61) as a section of Cochlodina, but on an equal footing with Clausilia, Pupa, and Cyclostoma. Hence there is room for doubt both as to the validity of Pupoides, Pfr., and as to whether it is actually equivalent to Lencochiloides, so that it may be advisable to retain for the present the later name, which is applied by most continental authorities to the species which follow.

With regard to the South African representation, specimens 
attributable to calaharicus, Bttg., have often been identified with the West African senegalensis, Morel., which was described from Goree. In my opinion, they very possibly are that species; but senegalensis was admitted by Morelet himself to be identical with the East Indian conopictus, Hutton, while the latter, with some twenty other names, has been placed by various authors in varying synonymy, including that of the American marginatus, Say (= fallax, auctt., nec Say).

It will hence be seen that the whole question of the synonymy of senegatensis is an extremely difficult one, and, as it is not yet settled, I prefer to leave this West Coast species altogether out of calculation, and restrict the South African list to the narrowest possible limits, as set forth below.

335. Leucochiloides calaharicus, Böttger.

1886 Buliminus (Leucochiloides) calaharicus, Bttg., Ber. Senckenb. Naturf. Ges. Frankfurt, p. 24. pl. 2, f. 3. D.F.

1900 Bulimimus (Leucochiloides) calaharicus, Bttg., Kob., Conch. Cab. p. 664. pl. 101, f. 22, 23. D.F.

Type in Senckenberg Mus. Frankfurt.

Hab. British Beohuanaland. Ghous (Nolte).

Griqualand West. Blaauwbosch Poort, Hay District (Day). Hartz River, Taungs (Miss Wilman).

Damaraland (Geale, in British Museum).

RHODESIs. Victoria Falls (Connolly).

CAPE of Good Hope. Jansenville (Farquhar; Crawford). Prieska (Gibbons). Karroo (in British Museum)

A variable species, both as to size and form.

336. Leucochiloides minusculus, Mousson.

1887 Buliminus (Lencochiloides) mimusculus, Mouss., J. de C. xxxv. p. 295. pl. 12, f. 5. D.F.

1902 „ (Pupoides) minusculus, Mouss., Kob., Conch. Cab. p. 956. pl. 133, f. 18, 19. D.F.

1904 " (Lencochiloides) minusculus, Mouss., von Mts., Die Kalahari, p. 754. N.

1910 Lencochiloides minusculus, Mouss., Bttg., Abh. Senckenb. Naturf. Ges. Frankfurt, xxxii. p. 445. N.

Type in Zurich Museum.

Hab. Ovampoland. Ku-Ganab, S.E. of Ondonga (Schinz). Hoeis (Hermann). Sodanna (Passarge). 
Bechuanaland. Meno a kwena (fossil, Passarge).

Separable by its smaller size, if a constant feature, from calaharicus, Bttg.

\section{Sub-Genus MICROSTELE, Böttger, 1886.}

(Ber. Senckenb. Naturf, Ges. Frankfurt, p. 26.)

Type of Sub-Genus, L. noltei, Bttg.

337. Leucochiloides noltei, Böttger.

1886 Pupa (Microstele) noltei, Bttg., Ber. Senckenb. Naturf. Ges.

Frankfurt, p. 25. pl. 2, f. 4. D.F.

1908 ", noltei, Bttg., M. \& P., A.M.N.H.i.p.78.pl.2, f.14, 15. D.F.

N.B.-The height line, which should be $3.75 \mathrm{~mm}$, is omitted on this plate.

Type in Senckenberg Museum, Frankfurt.

Hab. Brimish Bechuanaland. Ghous (Nolte).

Founded on a single specimen.

338. Leucochiloides oblongus, Böttger.

1910 Leucochiloides (Microstele) oblongus, Bttg., Abh. Senckenb. Naturf. Ges. Frankfurt, p. 445. pl. 28, f. 11. D.F.

Type in Senckenberg Museum, Frankfurt.

Hab. Damaranand. 140 kilom. inland from Swakopmund (Rintelen).

Founded on a single, live specimen, which, as the author recognises, may be a local form of noltei.

Genus JAMINIA, Leach in Risso, 1826.

(Hist. nat. de l'Europe mérid. iv. p. 88.)

(=Pupa, Drap., 1801, nec Pupa, Bolten, 1798, nec Lam., 1801.)

Type of Genus, J. muscomım (Müll.).

I have adopted the nomenclature suggested by B. B. Woodward in Journ. of Conch. x. 1903, p. 358 et seq. On p. 361 he points out that the Sub-genus Pupilla, Leach in Turton, 1831, by the adoption of Jaminia, becomes a synonym for Jaminia s.s.

$$
\text { 339. Jaminia bisuldata (Jickeli). }
$$

1872 Pupa bisulcata, Jick., Mal. Blätt. xx. p. 107. D.

$1874 \quad$ " $\quad$ Fauna N.-O.-Afr. p. 119.pl.5, f. 10. D.F. $1877 \quad$ „, „ $\quad$ Pfr., Mon. Hel. viii. p. 380. D.

Type in Zool. Mus. Berlin. 
Hab. Rhodesia. Victoria Falls (Connolly).

Described from Abyssinia.

It has not been possible to compare the Rhodesian shells with Jickeli's type, but they agree very well with his figure and description of bisulcata.

\section{Jaminia corrugata, Preston.}

1912 Jaminia cormuata, Prest., A.M.N.H. ix. pp. 70, f. 4; 71. F.D. Type in coll. Preston.

Hab. Rhodesia. Victoria Falls (Connolly).

341. Jaminia crawfordiana (Melv. \& Pons.).

1903 Fauxulus crawfordianus, M. \& P., A.M.N.H. xii. p. 605. pl. 31, f. 6 . D.F.

1908 Pupa cranfordiana, M. \& P., A.M.N.H. i. p. 71. N. 1911 , Bnp., A.M.N.H. vii. p. 402. pl. 10, f. 1, 2. N.F.

Type in British Museum.

Hab. Cape of Good Hope. Mossel Bay (Crawford).

342. Jaminia CRyptoplax (Melv. \& Pons.). [S.A.M.] 1899 Pupa cryptoplax, M. \& P., A.M.N.H. iv.p. 198. pl. 3, f. 11. D.F. $1908 \quad$ " $\quad$ " i. p. 71. pl. 1, f. 1,2. N.F. $1911^{\circ} \quad$ ", Bnp., A.M.N.H. vii. p. $402 . \quad$ N.

Type in British Museum.

Hab. CAPE of Good Hope. Kragga Kama, Port Elizabeth (Crawford ; Reeve).

\section{JaMinia DADION (Benson).}

1864 Pupa dadion, Bs., A.M.N.H. xiii. p. 495. D.

$1868 \quad$ " " Pfr., Mon. Hel. vi. p. 320. D.

$1908 \quad$ " $\quad$ M. \& P., A.M.N.H. i. p. 72. pl. 1, f. 3. N.F.

Type in British Museum.

Hab. Cape of Good Hope. Simonstown; Ravine near Newlands (Layard). Bedford (Farquhar).

Natad. Umvoti Country (Lightfoot). Karkloof; Nottingham Road; Inhluzani Mountain; Game Pass (Burnup).

\section{Jaminia Damarica (Ancey).}

1888 Pupa dancorica, Ancey, Le Naturaliste, x. p. 200. D.

1892. " ovampocnsis, M. \& P., A.M.N.H. ix. p.91. pl. 6, f. 11. D.F. 
1901 Pupa ridibunda, M. \& P., A.M.N.H. viii. p. 320. pl. 2, f. 11. D.F. 1908 „ damarica, Ancey, M. \& P., A.M.N.H. i. p. 72. D.

" " ovampoensis, M. \& P. (=ridibunda), M. \& P., A.M.N.H. i. p. 79. pl. 2, f. 16 . D.F.

1910 Leucochilus damaricum, Ancey (=ovampoensis, M. \& P.), Bttg., Abh. Senckenb. Naturf. Ges. Frankfurt, xxxii. p. $446 . N$. 1911 Pupa damarica, Ancey, Bnp., A.M.N.H. vii. p. $403 . \quad$ N.

Type of damarica-ubi?; ridibunda and ovampoensis in British Museum.

Hab. Ovampoland (ovampoensis, in coll. Layard). Disappoint. ment Vlei (damarica, Andersson \& Chapman).

Transvaal. Rustenberg (McBean). Potchefstroom (Miss Livingston). Pretoria; Heidelberg; Buiskop; Pietersburg; Pruizen (Connolly).

Orange Free State. Bloemfontein (Connolly).

Cape of Goon Hope. Prieska (Gibbons). Elandsberg Mountain, Cradock (ridibunda); Port Elizabeth (Farquhar).

345. Jaminia dysorata (Melv. \& Pons.).

1893 Prpa dysorata, M. \& P., A.M.N.H. xi. p. 20. pl.3,f.4. F. and faulty description.

\begin{tabular}{|c|c|c|c|c|}
\hline 89 & & dysorot & & oll. p. 71. (Err.typ.) \\
\hline & & dysorata & & I.N.H. i. p. 73. pl. 1, f. 4. D.F. \\
\hline & & & " & H. vii. p. $403 . \quad$ N.I \\
\hline
\end{tabular}

Type in coll. Sykes.

Hab. CAPE of Good Hope. Griqualand East (in coll. Sykes).

346. Jaminia farquhari (Melv. \& Pons.).

1898 Pupa farquhari, M. \& P., A.M.N.H. ii. p. 128. pl. 7, f. 7. D.F. 1908

Type in British Museum.

Hab. CAPE OF Good Hope. Elandsberg Mountain, Cradock (Farquhar).

347. Jaminia fontana (Krauss).

1841 Prupa fontana, Krs., Küst., Conch. Cab.p.122.pl.16, f.9-12. D.F. 1848 Südafr. Moll. p. 80. pl. 5, f. 6. D.F.

",

1866

" Pfr., Mon. Hel. ii. p. 355. D.

1870 von Mts., Mal. Blätt. xiii. p. $96 . \quad N$. 1874

" Blanf., Obs. Geol. \& Zool. Abyss. p. 477. N. " Jick., Fauna N.-O.-Afr.p.120.pl.5,f.11. D.F. 
1894 Pupa charybdica, M. \& P., A.M.N.H. xiv. p. 94. pl. 1, f. 13. D.F.

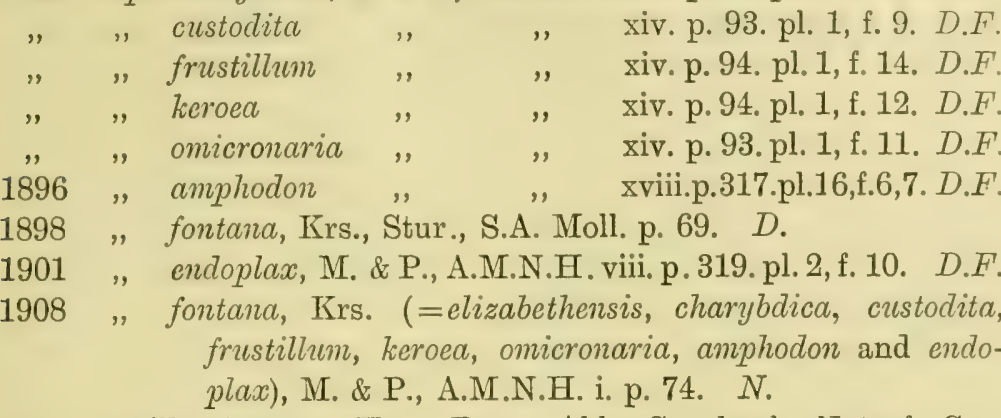

1910 Pupilla fontana, Krs., Bttg., Abh. Senckenb. Naturf. Ges. Frankfurt, xxxii. p. 445. N.

1911 Pupa fontana, Krs., Bnp., A.M.N.H. vii. p. 404. N.

Types of amphodon, charybdica, custodita, endoplax, frustillum, keroea, and omicronaria in British Museum; fontana in Stuttgart Museum.

Hab. Transvaat. Source of Mooi River (Wablberg). Pretoria District (custodita, keroea, omicronaria, \&c., very plentiful). Johannesburg (McBean; Johnson). Potchefstroom; Heidelberg (Miss Livingston). Buiskop (Connolly).

Orange Free State. Bloemfontein; Rustfontein (Connolly).

NataL. Karkloof (McBean). Edendale; Tongaat (Burnup).

CAPE of Good Hope. Prieska (Lightfoot). Cradock (endoplax, Farquhar). Port Elizabeth (amphodon, charybdica, frustillum, Crawford; Penther). East London (Miss Bowker). Victoria East; Pirie Forest (Godfrey).

Griqualand West. Blaauwbosch Poort, Hay District (Day).

Damaraland. Gobabis (sub-fossil, Hermann).

Also known from Abyssinia and other countries. Nevill cites a variety of this species as collected by Blanford at Agula, Adignat, and Meshek, in North-East Africa. Jickeli (1874) describes and figures a var. globulosa, which he collected in Abyssinia in company with the typical form.

var. elizabethensis (Melv. \& Pons.).

1892 Pupa elizabethensis, M. \& P., A.M.N.H. ix. p. 91. pl. 5, f. 13. D.F. Type in British Museum.

Hab. Cape of Good Hope. Port Elizabeth (Crawford).

The albino form of fontana, and, as such, entitled to varietal rank. 
348. Jaminia Griqualandica (Melv. \& Pons.). [S.A.M.] 1893 Pupa griqualandica, M. \& P., A.M.N.H. xi.p. 22. pl. 3, f. 9. D.F. $1908 \quad$ " $\quad$ " $\quad$ i.p.76.pl.1,f. 8-10. D.F. $1911 \quad$ ", " Bnp., A.M.N.H. vii. p. 405. N.

Type in coll. Sykes.

Hab. CaPe of Good Hope. Griqualand East (Sykes). Cradock (Farquhar). Port Elizabeth (in coll. Ponsonby).

Natal. Pietermaritzburg; Dargle; Tongaat; Edendale (Burnup).

Zululand. Dukuduku (Toppin).

Transvaal. Pretoria District (Farquhar). Heidelberg (Miss Livingston). Buiskop (Connolly).

349. Jaminia тота (Melv. \& Pons.). [S.A.M.]
[. 1894 Pupa iota, M. \& P., A.M.N.H. xiv. p. 93. pl. 1, f. 10. D.F. 1908 " " $\quad$ i. p. 77. pl.1, f. 11. D.F. $1911 \quad " \quad$ " Bnp., A.M.N.H. vii. p. 406. N.

Type in British Museum.

Hab. Transvadu. Pretoria (Farquhar; McBean). Heidelberg (Miss Livingston). Standers Kop (Connolly).

Zululand. Dukuduku Forest (Toppin).

$$
\text { var. LIVINGSTONæe, Burnup. }
$$

1908 Pupa iota, M. \& P., var. livingstona, Bnp., M. \& P., A.M.N.H. i. p. 77. pl. 1, f. 12. D.F.

Type in British Museum.

Hab. Transvaal. Pretoria; Standerton (Connolly).

350. Jaminia LAXARdi (Benson).

1856 Pupa layardi, Bs., A.M.N.H. xviii. p. 435. D.

1859

1864

1868

1876

1908

1911
", Pfr., Mon. Hel. iv. p. 674. D.

"A.M.N.H. xiii. p. $496 . \quad N$.

" Pfr., Mon. Hel. vi. p. 318. D.

" Sow., Conch. Icon. pl. 15, f. 141. D.F.

" M. \& P., A.M.N.H. i. p. 78. pl. 2, f. 13. N.F.

„, Bnp., A.M.N.H. vii.p.407.pl. 10, f. 3, 4. N.F.

Originals in University Museum of Zoology, Cambridge.

Hab. Cape of Good Hope. Cape Point (Layard). Hermanus (Lightfoot).

var. Mrinon, Benson.

1864 Pupa layardi, var. minor, Bs., A.M.N.H. xiii. p. 496. D. 1868 " Pfr., Mon. Hel. vi.p. 318. D. 
Pupa stoaphora, Bs., in litt.

1889 " " " Paetel, Catalog, ii. p. 305.

1908 " layardi, var. minor, Bs., M. \& P., A.M.N.H. i.p.78. N.

1911 " " " Bnp., A.M.N.H. vii. p. 408. pl. 10, f. 5, 6. N.F.

Originals of var. minor in University Museum of Zoology, Cambridge; stoaphora in British Museum.

Hab. CAPE OF GooD Hope. Bredasdorp, at the roots of grasses among stones (stoaphora, Layard).

\section{Jaminia Perplexa (Burnup). [S.A.M.]}

1908 Pupa perplexa, Bnp., M.\& P., A.M.N.H.i.p.80.pl.2,f.17,18. D.F. $1911 \quad$ " $\quad$ A.M.N.H. vii. p. 408. D.

Type in British Museum.

Hab. CAPE of Good Hope. Cradock; Port Elizabeth (F'arquhar).

Transvaal. Johannesburg (MoBean). Pretoria (in coll. Ponsonby). Potchefstroom (Miss Livingston), Heidelberg (Connolly).

Orange Free State. Bloemfontein (Connolly).

352. Jaminia pretoriensis (Melv. \& Pons.). [S.A.M.] 1893 Pupa pretoriensis, M. \& P., A.M.N.H. xi. p. 21. pl. 3, f. 8. D.F. 1908 i. p. $81 . N$.

" " dysorata, M. \& P., var. intradentata, Bnp., M. \& P., A.M.N.H. i. p. 73. pl. 1, f. 5, 6. D.F.

1911 „, intradentata, Bnp., A.M.N.H. vii. p. 405. D.

Types in British Museum.

Hab. Transvaal. Pretoria and District (Farquhar; Connolly).

The type of pretoriensis, which was not available when the revision of the South African Pupida was being prepared (1908), proves to be a slightly immature example of the species better known as intradentata, Bnp. Pretoriensis, of course, has priority.

353. Jaminia quantula (Melv. \& Pons.).

1893 Pupa quantula, M. \& P., A.M.N.H. xi. p. 20. pl. 3, f. 5. D.F. 1908.

1911

Type-ubi?

i. p. 81. pl. 2, f. 19. N.F.

Hab. CaPe of Goon Hope. Port Elizabeth, South of Baakens River to Schoenmakers Kop (Crawford). 
1893 Prpa sykesii, M. \& P., A.M.N.H. xi. p. 21. pl. 3, f. 6. F. $\&$ faulty description.

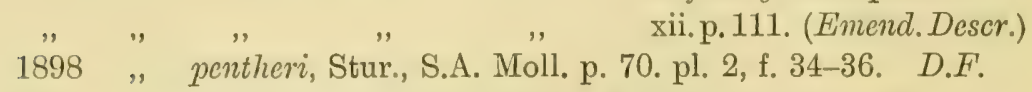
1908 " , " M. \& P., A.M.N.H. i. p. 80. N. , " $\quad$ sykesii, M. \& P., ibid. p. 81. pl. 2, f. 20. N.F.

1911 ", sykesi " (=pentheri, Stur.), Bnp., A.M.N.H.vii. p. $410 . N$.

Type of sykesi in coll. Sykes; pentheri in Naturh. Hofmus. Vienna. Hab. Cape of Good Hope. Griqualand East (in coll. Sykes). Port Elizabeth (Reeve). Pirie (Godfrey). Grahamstown (Farquhar).

Natal. Majuba (Connolly). Durban; Umbilo Road (pentheri, Penther). Edendale; Game Pass ; Ntimbankulu (Burnup).

Zululand. Dukuduku (pentheri, Toppin).

var. Inconspicua, Burnup.

1908 Pupa sykesii, M. \& P., var. inconspicua, Bnp., M.\& P., A.M.N.H. 1911 i. p. 81.pl. 2, f.21. D.F.

Type in British Museum. Bnp., A.M.N.H.vii.p.410. N.

Hab. Natal. Dargle (Miss Livingston).

355. Jaminia tabularis (Melv. \& Pons.). [S.A.M.] 1893 Pupa tabularis, M. \& P., A.M.N.H. xi. p. 20. pl. 3, f. 3. D.F. 1908 i. p. 82. pl. 2, f. 22 . N.F.

Type in British Museum.

Hab. Cape Peninsula. Cape Town (Lightfoot). Rondebosch (Connolly).

356. Jaminia tetrodus (Böttger).

1870 Pupa tetrodus, Bttg., Ber. Offenbach. Ver. f. Naturk. xi. p. 46. pl. 1, f. 1. D.F.

1880 , (Vertigo) sinistrorsa, Crvn., P.Z.S. p. 618. pl. 57, f. 8. D.F. 1891 " " $\quad$ thaumasta, M. \& P., A.M.N.H. viii. p. 239. D. $1892 \quad$ " " " , $\quad$ ix. p. 94. pl. 6, $1898 \quad$ " $\quad$, $\quad$ f. $7 . \quad F$. $1908 \quad$ " $\quad$ sinistrorsa, Crvn.(=thaumasta),M.\&P.,A.M.N.H. i. p. 83. pl. 2, f. $26 . \quad$ N.F. 
1910 Pupilla tetrodus, Bttg. (=sinistrorsa and thaumasta), Bttg., Abb. Senckenb. Naturf. Ges. Frankfurt, xxxii. p. $446 . \quad N$. Types of sinistrorsa and thanmasta in British Museum; tetrodus in Senckenberg Museum, Frankfurt.

Hab. Bechuanaland. Gokwe River, $22^{\circ} \mathrm{S}$. lat. and $28^{\circ} \mathrm{E}$. lon., about 30 miles $N$. of Palapye Road (sub-fossil, Hübner).

Cape of Goon Hope. Cape Récif; Klein Setjes Bosch, near Beaufort (sinistrorsa, Craven). Port Alfred (Penther). King Williamstown; Lovedale; Burns Hill (Godfrey). Port Elizabeth (thaumasta, Crawford). Prieska (Gibbons). Grahamstown; Cradock ; Jansenville; Somerset East (Farquhar). Coega (Miss Hickey).

British Bechuanaland. Hartz River, Taungs (Miss Wilman).

Transvaal. Pretoria (Connolly). Potchefstroom (Miss Livingston).

Orange Free State. Bloemfontein (Godfrey). Kroonstad (Miss Hickey).

Ovampoland. Disappointment Vlei (in coll. Layard).

Sub-Genus FAUXULUS, Schaufuss, 1869.

(Paetel's Catalog, p. 15.)

(=Faula, H. \& A. Adams, 1855, nec Blanchard, 1850.)

Type of Sub-Genus, J. capensis (Kurr).

357. Jaminia (Fauxulus) capensis (Kurr).

1841 Pupa capensis, Kurr, Küst., Conch. Cab. p.10.pl.1, f.19,20. D.F.

" " ovularis ", ", ibid.p. 10. pl. 1, f. 16-18. D.F.

" " $\quad$ pottebergensis, Krs., Küst., ibid. p. 17. pl. 2, f. 20-22. D.F.

" " kurrii, Krs. (=ovularis, Kurr, nec Oliv.), Küst., ibid.

p. 111. pl. 15, f. 5, 6. D.F.

1842 ", capensis, Kurr, Pfr., Symb. ii. p. 53. D.

" " kurrii and pottebergensis, Krs., Pfr., ibid. p. 54. D.

1848 ", capensis, Kurr, kurrii and pottebergensis, Krs., Pfr., Mon.

Hel. ii. p. 331. $D$.

1851 " fonticola, Desh., Hist. nat. Moll. ii. p. 220. pl. 156, f. 26-

28. D.F.

1878 " pottebergensis, Krs., Sow., Conch. Icon. pl. 18, f. 166. D.F. " " kurrii, Krs. (=fonticola), Sow., ibid. pl. 19, f. 182. D.F.

1898 " pottenbergensis, Krs., Stur., S.A. Moll. p. 67. (Err.typ.)

1908 ", capensis, Kurr (cum varr. kurri and pottebergensis, Krs.), M. \& P., A.M.N.H. i. p. 83.

1911 " " " (=kurri and pottebergensis), Bnp., A.M.N.H. vii. p. 411. N. 
Types of capensis, kurvii, and pottebergensis in Stuttgart Museum.

Hab. CAPE of Good Hope. Zoetendals Valley and Potteberg, Swellendam District (Krauss). Port Elizabeth (Crawford). Gordon's Bay (Connolly). St. Helena Bay; recent and fossil at Saldanha Bay (Lightfoot).

British Beohuanaland. Kuruman (Moffatt, fide Layard).

Cape Peninsula. Widely distributed. A pretty variety with bright yellow brown band below the suture and yellow base comes from Buffelsfontein, Cape Point, where shells of uniform pale orange colour are also found.

Fonticola was described from "Cape of Good Hope" (Verreaux).

358. Jaminia (Fauxulus) fryana (Benson).

1864 Pupa fryana, Bs., A.M.N.H. xiii. p. 495. D.

$1868 \quad$ " $\quad$ " Pfr., Mon. Hel. vi. p. 319. D.

1908 Pupa (Fauxulus) fryana, Bs., M. \& P., A.M.N.H. i. p. 83. N.

Type in British Museum.

Hab. Cape of Good Hope. Bredasdorp (Fry; At the roots of grasses among stones, Layard).

\section{Jaminia (Fauxulus) glanvilleana (Ancey).}

1888 Pupa glanvilliana, Ancey, Le Naturaliste, x. p. 200. D.

1908 " (Fauxulus) glanvilleana,Ancey,M.\&P.,A.M.N.H.i.p.83. D. 1911 " , , " , Bnp., A.M.N.H. vii.p. 411. $N$.

Type-ubi?

Hab. Cape of Good Hope. East London (Miss Glanville; Miss Bowker):

var. DARGLENSIS, Burnup.

1908 Pupa (Fauxulus) glanvilleana, Ancey, M. \& P., A.M.N.H.i. pl. 2, f. $23 . F$.

1911

,

Ancey,var.darglensis,Bnp.,A.M.N.H.

Type in British Museum. vii. p. 412 . $D$.

Hab. Natal. Dargle; Game Pass, near Giant's Castle, Drakensberg; Inhluzani Mountain; Karkloof; Ntimbankulu (Burnup).

var. Toulini, Burnup.

1911 Pupa (Fauxulus) glanvilleana, Ancey, var tomlini, Bnp., A.M.N.H. vii. p. 413. pl. 10, f. 7. D.F.

Type in British Museum. 
Hab. Cape of Good Hope. Albany (Miss Glanville). East London (Radford). Gamtoos (Reeve).

360. Jaminia (Fauxulus) mcreaniana, Melv. \& Pons. [S.A.M.] 1901 Fauxulus (Anisoloma) mobeanianus, M. \& P., A.M.N.H. viii. p. 319 . pl. 2, f. 9 . D.F.

1911 Pupa (Fauxulus) mebeaniana, M. \& P., Bup., A.M.N.H. vii. p. 414. pl. 10, f. 8. N.F.

Type in British Museum.

Hab. Natal. Karkloof Bush (McBean). Majuba (Connolly). Dargle; Nottingham Road (Burnup).

361. Jaminia (Fauxulus) pamphorodon (Benson). [S.A.M.]
[S. 1864 Pupa pamphorodon, Bs., A.M.N.H. xiii. p. $495 . \quad D$. $1868 \quad$ " $\quad$, $\quad$ " Pfr., Mon. Hel. vi. p. 320. D. $1876 \quad$ " " $\quad$ "Sow., Conch. Icon. pl. 13, f. 120. D.F. 1908 ", (Fauxulus) pamphorodon, Bs., M. \& P., A.M.N.H. i. p. 84. pl. 2, f. 24. N.F.

1911

Type in British Museum. ,, Bnp.,A.M.N.H.vii.p.414.N.

Hab. Cape Peninsula. Simonstown (Layard). Kalk Bay; Slang Kop; Paul Berg; Cape Point (Connolly).

362. Jaminia (Fauxulus) pereximia (Melv. \& Pons.). 1897 Pupa (Faula) pereximia, M. \& P., A.M.N.H. xix. p. 638. pl. 17, f. 3. D.F.

1908 " (Fauxulus) pereximia, M. \& P., A.M.N.H. i.p. 85. pl. 2, f. $25 . N . F$.

Type in British Museum.

Hab. Cape of Good Hope. Buffalo River (fide M. \& P.).

363. Jaminia (Fauxulus) ponsonbyana (Morelet).

1889 Pupa (Faula) ponsonbyana, Morel.,J.de C.xxxvii.p.9.pl.1,f.5. D.F. 1901 „, (Anisoloma) ponsonbyana, Morel., Ancey, J. de C. xlix. p. 140. N.

1908 ", (Fauxulus) ponsonbyana,MLorel.,M.\&P.,A.M.N.H.i.p.85. N. 1911 , " , , , Bnp., A.M.N.H. vii. p.415.

Type in British Museum. pl. 10, f. 9-12. N.F.

Hab. Cape of Good Hope. Port Elizabeth (Crawford; Reeve). 
Somerset East (Miss Bowker). Grahamstown; Bathurst; Kowie (Farquhar). Alexandria District (Crawford). Pirie (Godfrey).

NATAL. Hilton Road; Zwaart Kop, near Pietermaritzburg (Burnup).

FAmily CLAUSILIIDA, B. B. Woodward, 1903. (Journ. of Conch. X. pp. 355, 361.)

Genus BALEA (Prideaux MSS.), Gray, 1824.

(Zool. Journ. i. p. 61.)

Type of Genus, B. fragilis, Drap. (perversa, Lin.).

364. Balea africana, Melv. \& Pons.

[S.A.M.]

1899 Balea africana, M. \& P., A.M.N.H. iv. p. 198. pl. 3, f. 10. D.F.

Type in British Museum.

Hab. Natal. Van Reenen (Quekett). Karkloof (Burnup).

Cape of Good Hope. Bedford (Farquhar). Pirie Forest (Godfrey).

FAmily ACHATINID E, von Martens, 1879.

(Zoological Record, Moll. p. 65.)

(=Achatinida, Pfr., 1879, Nomenclator, p. 260.)

Sub-Family ACHATININ E, H. \& A. Adams, 1855.

(Gen. rec. Moll, ii. p. 131.)

Genus MeTACHATINA, Pilsbry, 1904.

(Man. of Conch. xvi. p. 307.)

Type of Genus, M. kraussi (Pfr.).

365. Metachatina kraussi (Pfeiffer).

[S.A.M.]

1846 Bulimus kraussi, Pfr., Symb. iii. p. 85. D.

$1848 \quad$ " $\quad$ " Krs., Südafr. Moll. p. 78. pl.5, f. 4. D.F.

$\begin{array}{llll}\prime \prime & \text { " } & \text { ", Mon. Hel. ii. p. 184. D. } & \\ 1849 & , & \text {, } & \text { Rve., Conch. Icon. pl. 63, f. 436. D.F. }\end{array}$

1860 Achatina fuscolabris, von Mts., Die Helic. pp. 202, $204 . \quad$ N.

1889 " (Butimus kraussi, Pfr.), von Mts., Sitz.-

Ber. Ges. Nat. Fr. Berlin, p. 163. N.

, Livinhacia kranssi, Pfr. (=fuscolabris, von Mts.), Crosse, J. de

C xxxvii. p. 111 . D. 
Reference List of South African Non-marine Mollusca. 159

1893 Livinhacia kranssi, Pfr., Kob., Conch. Cab. p. 7. pl. 2, f. 1. D.F. 1899 , Junod, Bull. Soc. Vaudoise, xxxy. p. 279. N.

1904 Metachatina kraussi, Pfr., Pilsb., Man. of Conch. xvi. p. 308. pl. 23, f. 46,48 . D.F.

Type in Stuttgart Museum.

Hab. Natal. Natal Bay (Krauss; Penther). Woods near the Umlaas River (fide Pfeiffer). Tongaat; Alexandra (now renamed Kelso) Junction (Burnup).

Lorenzo Marques. Rikatla (Junod).

Eastern Zululand (Toppin).

var. PLanti, Pfeiffer.

1861 Achatina planti, Pfr., P.Z.S. p. 25. pl. 3, f. 6. D.F.

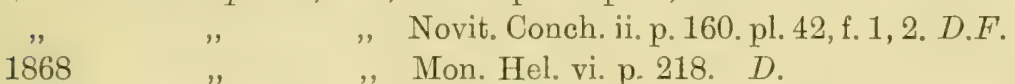

1898 Livinhacia kraussi,var.planti,Pfr.,M.\&P.,Proc.Mal.Soc.iii.p.178.

1904 Metachatina planti, Pfr., Pilsb., Man. of Conch. xvi. p. 309. pl. 37, f. 10,11 . D.F.

Type in British Museum.

Hab. Natal. Cape Natal (Plant).

Zululand. Kosi Bay (Toppin).

Genus BURTOA, Bourguignat, 1889.

(Moll. de l'Afr. équat., March, 1889, p. 88.)

(=Livinhacia, Crosse, April, 1889).

Type of Genus, B. nilotica (Pfr.).

366. Burtoa nilotica (Pfeiffer).

[S.A.M.]

1861 Bulimus niloticus, Pfr., P.Z.S. p. 24. D.

$1868 \quad$ " $\quad$, " Mal. Blätt. viii. p. $14 . \quad$ D.

1870 Limicolaria nilotica, Pfr., Novit. Conch.iv. p.5. pl.110, f.1-3. D.F. Achatina nilotica, Pfr., von Mts., Mal. Blätt. xvii. p. $32 . N$.

1874

1889 Burtoa " $\quad$ " Bgt., Moll. de l'Afr. équat. p. 89. N.

" Livinhacia ", " Crosse, J. de C. xxxvii. p. 109. D.

1891 Achatina (Livinhacia) nilotica, Pfr., von Mts., Sitz.-Ber. Ges.

Nat. Fr. Berlin, p. 14. N.

1893 Livinhacia nilotica, Pfr., Kob., Conch. Cab. p. 5. pl. 1, f. 1. D.F.

1895 Burtoa

" Smith, Proc. Mal. Soc. i. p. 323. N. 
1897 Limicolaria (Livinhacia) nilotica, Pfr., von Mts., D.-O.-Afr. pp. 94-98. D.F. Varr. and Synonymy.

1898 Livinhacia amoldi, Stur., S.A. Moll. p. 59. pl. 2, f. 41. D.F. 1904 Burtoa amoldi, Stur., Pilsb., Man. of Conch. xvi. p. 307. pl. 26, f. 1. I). $F$.

, = , nilotica, Pfr., Pilsb., ibid. p. 300. pl. 26, f. 15, 16. pl. 27, f. 5, 6. pl. 29 , f. 7, 8. pl. 30, f. 18. pl. 35, f. 22, 23. D.F. 1906 „, nilotica, Pfr., Reynell, Proc. Mal. Soc. vii.p. 197. pl. 17, f. 1-3. A.R.

1907 Burtoa nilotica, Pfr. (=amoldi, Stur.), Melv. \& Standen, Manchester Memoirs, li, 4. p. 11. N.

Type of nilotica in British Nuseum; arnoldi in Naturh. Hofmus. Vienna.

Hab. Matebililand. Near the Amanze Inyama River (arnoldi, Penther).

Rhodesia. Salisbury (Oakley). Victoria Falls (Soper). Insiza (French).

A most variable form, originally described from The Source of the White Nile (Petherick), and occurring in many parts of Eastern Africa. Crosse (1889) places Bul. giraudi, Bgt., in the synonymy, to which von Martens (1897) adds jouberti, sebasmia, bridouxiana, and lavigeriana, Bgt. The last-named author also identifies pethericki, Bgt., with var. schweinfurthi, von Mts., and describes varr. emini, crassa, obliqua, and oblonga. Pilsbry (1904) brings in as further varieties reymondi, Bgt., and grundidieri, Pilsb. (=Limicolaria bourguignati, Grandidier).

Genus ACHATINA, Lamarck, 1799.

(Mém. Soc. Hist. Nat. Paris, p. 75.)

(=Oncœa, Gistel, 1850; Urceus (Klein), Jousseaume, 1884, and Parachatina, Serpcea, and Pintoa, Bourguignat, 1889.)

Type of Genus, A. achatina (Lin.).

Pilsbry (1904) places most of the South African forms previously attributed to this Genus in Cochlitoma, Fér. (Tabl. Syst. Moll. pt. 3, 1821, pp. 28, 52 (or 24, 48), Type A. zebra, Chem.), and Kobelt (1909) follows his example. Apparently there are no anatomical grounds for the subdivision, which rests on a very fragile conchological basis. As many of the leading British and South African authorities are averse to the change, I prefer to retain the older classification. 
367. Achatina ampullacea, Böttger.

1910 Achatina ampullacea, Bttg., Abh. Senckenb. Naturf. Ges. Frankfurt, xxxii. p. 447. pl. 28, f. 13. D.F.

Type in Senckenberg Mus. Frankfurt.

Hab. Bechuanaland. Banks of the Epukiro-Omuramba, 15 kilom. from Komeduve, south of Lake Ngami (Hermann).

A single specimen, likened by Böttger to A.damarensis, which, as he says, is found only 5 miles distant from it.

368. Achatina aurora, Pfeiffer.

1854 Achatina aurora, Pfr., P.Z.S. p. 294. D.

$1859 \quad$ " $\quad$ Mon. Hel. iv. p. 602. D.

1904 Cochlitoma aurora, Pfr., Pilsb., Man. of Conch. xvii. p. 102. D.N.

Type in British Museum.

Hab. Natal. Durban (coll. Cuming).

The type, and only known specimen, is a semi-bleached, beachrolled shell, recalling a West African rather than South African form, which may quite possibly have been dropped at Durban in ballast. It has not yet, however, been identified with any other known species.

369. AChatina Bisculpta, Smith.

1878 Achatina bisculpta, Smith, Quart. Journ. of Conch. i. p. 349. D. $1902 \quad$ " , , , Ancey, J. de C. 1. p. 280, f. 8. N.F. 1904 Cochlitoma , , , Pilsb.,Man.of Conch.xvii.p.94.pl.13, f. 44. D.F.

Type in British Museum.

Hab. "South Africa" (in British Museum).

370. Achatina Burnupi, Smith.

1890 Achatina burnupi, Smith, A.M.N.H. vi. pp. 392, 393. D.

1904, 5. Cochlitoma burmupi, Smith, Pilsb Man. of Conch. xvii. p. 97. pl. 32 , f. 5. D.F.

Type in British Museun.

Hab. Natal. Van Reenen's Pass, Drakensberg (Burnup). Giant's Castle (Mann).

Transvaal, Carolina District (Horsbrugh).

371. Achatina churchilliana, Melv. \& Pons.

1895 Achatina churchilliana,M.\&P.,A.M.N.H.xv.p.164.pl.12,f.3. D.F. 1904, 5 Cochlitoma churchilliana, M. \& P., Pilsb., Man. of Conch. xvii. p. 101. pl. 33, f. 7, 10, D.F. 
Type in Manchester Museum.

Hab. NataL. "Port Natal" (Churchill; Grout).

372. Achatina cinnamonea, Melv. \& Pons.

1894 Achatina cinnamomea, M.\&P., A.M.N.H.xiv. p.92. pl. 1, f.6. D.F. 1904, 5 Cochlitoma cinnamomea, M. \& P., Pilsb., Man. of Conch. xvii. p. 94. pl. 29, f. 42 . D.F.

Type in Natal Museum, Pietermaritzburg.

Hab. Transvaal. Standerton (Burnup).

373. Aсhatina connoliyi, Preston.

[S.A.M.]

1912 Achatina connollyi, Prest., A.M.N.H. ix. p. 71, f. 5. D.F.

Type in coll. Preston.

Hab. Rhodesia. Victoria Falls (Connolly).

374. Achatina crawford, Morelet.

[S.A.M.]

1889 Achatina craufordi, Morel., J. de C. xxxvii. p. 8. pl.1, f. 3. D.F. $1897 \quad$ " $\quad$ " Clapp, Nautilus, xi. p. 69. N.

$1902 \quad$ " $\quad$ " Smith, Proc. Mal. Soc. v. p. 169. N. 1904, 5 Cochlitoma crawfordi, Morel., Pilsb., Man. of Conch. xvii. p. 93. pl. 26, f. 27,28 . pl. 64 , f. $69-71$. D.F.A.

Type in British Museum.

Hab. Cape of Good Hope. Middleton; Kleinpoort (Crawford). Somerset East; Bedford (Farquhar). Douglas (Miss Orpen).

Morelet's original locality, Port Elizabeth, is incorrect.

375. Achatina damarensis, Pfeiffer.

1870 Achatina dammarensis, Pfr., Mal. Blätt. xvii. p. 31. D.

\begin{tabular}{|c|c|c|c|c|}
\hline " & ", & " & $"$, & $\begin{array}{l}\text { Novit. Conch. iv. p. 2. pl. 109, } \\
\text { f. } 3,4 \text { L.F. }\end{array}$ \\
\hline 87 & ", & ", &, & Mon. Hel. viii. p. 274. $D$. \\
\hline 904 & , & , & $"$ & $\begin{array}{l}\text { Pilsb., Man. of Conch. xvii. p. } \\
\text { pl. } 11, \text { f. } 32,33 \text {. D.F. }\end{array}$ \\
\hline & $"$ & damarensis, & $"$ & $\begin{array}{l}\text { Bttg., Abh. Senckenb. Naturf. G } \\
\text { Frankfurt, xxxii. p. } 446 . \quad N \text {. }\end{array}$ \\
\hline
\end{tabular}

Type in Stettin Museum.

Hab. Damaraland (Dohrn). Ubeb on the Khan River (Schenck). Omuramba-Omatuko River, near Okosongoho; Bank of the Black Nosob, near Gobabis (Hermann). 
Bechuanaland, Banks of the Epukiro-Omuramba, 10 kilom. east of Komeduve, south of Lake Ngami (Hermann).

Great Namadand. Choarib; Choa's District (Hermann).

\section{Achatina dimidiata, Smith.}

1878 Achatina dimidiata, Smith, Quart., Journ. of Conch. i.p.348. D. 1904, 5 Cochlitoma dimidiata, Smith, Pilsb., Man. of Conch. xvii. p. 95. pl. 32 , f. 6 . D.F.

Type in British Museum.

Hab. Transvaal. Lydenburg (in British Museum; Craven). Piet Retief (Crawshay).

Natal. Majuba (Connolly).

Orange Free State. Near Kopjes Siding (Connolly).

377. Achatina fulica (Férussac).

1821 Helix (Cochlitoma) fulica, Fér., Tabl. Syst. Moll. pt. 3. p.53 (or 49). , borbonica, Fér., ibid. p. 53 (or 49).

" " " $\quad$ " $\quad$ ", zebrina, Fér., ibid. p. 53 (or 49).

1822 Achatina mauritiana, Lam. (=fulica, Fér.), Lam., Hist. nat.

An. s. Vert. vi, 2. p. $129 . \quad D$.

1830 " couropa, Lesson, Voy. autour du Monde, Zool. ii. p. 318. pl. 9, f. 2. D.F.

1832 " mauritiana, Lam., Quoy \& Gaim., Voy. Astrolabe, Moll. p. 152. pl. 11, f. 10-15. pl. 49, f. 21. D.F.A. 1838 " mauritiana, Lam. (fulica, Fér.), Desh., Hist. nat. An. s. Vert. viii. p. 297. D.

1848 ", fulica, Fér., Pfr., Mon. Hel. ii. p. 254. D.

$1849 \quad$ ", " Rve., Conch. Icon. pl. 2, f. 8. D.F. $\quad " \quad \quad \quad \quad \quad \quad \quad \quad$ (=couropa, Less.), Phil., Abb. u. Beschr.
$\quad$ iii. p. 30. pl. 21, 1, f. 3. D.F.

1851 ,. ," ," (=borbonica and mauritiana), Desh., Hist. Nat. Moll. ii, 2. p. 162. pl. 124A, f. 1. pl. 125 , f. $3-5$. D.F.

1869 , , , , von Mts., von der Decken's Reisen, iii. p. 58. pl. 2, f. 1 . N.F.

1870 , „, „, Semp., Reis. im Arch. Philippin. ii, 3. p. 143 . pl. 12, f. 17 . A.

1880 , , , Mts., Meeresfauna von Mauritius, p. 197. $N$.

1892

Brancsik, Jahrb. Naturwiss. Vereins Trencsiner Com.xv.p. 204.pl.6, f. 6. A. von Mts., D.-O.-Afr. p. $89 . \quad N$. 
1904 Achatina fulica, Fér., Pilsb., Man. of Conch. xvii. p. 55. pl. 36, f. 18-20. pl. 37, f. 21-24. D.F.

Type-ubi?

Hab. Unknown to Férussac.

Natal. Durban (Burnup).

A Mauritian species, distributed over East Africa and the neighbouring islands of the Indian Ocean. Its presence in Durban has been traced to introduction in flower-pots from Mauritius. von Martens considers A. fulva (Brug.) and acuta, Lam., to be nearly related to fulica.

378. Aсhatina guutinosa, Pfeiffer.

1952 Achatina glutinosa, Pfr., P.Z.S. p. 86. D.

$1853 \quad$ ", " , Mon. Hel. iii. p. 485. D.

$1854 \quad$ " $\quad$ " $\quad$ A.M.N.H. xiii. p. 494 . D.

1859 " petersi, von Mts., Mal. Blätt. vi. p. 214. D.

$1860 \quad$ " " , " Die Helic. p. 294. N.

1865 " glutinosa, Pfr., Conch.Cab.p.360.pl.44(1854),f.1. D.F.

1868 ", petersi, von Mts., Pfr., Mon. Hel. vi. p. 213. D.

$1869 \quad$ " " , , " Novit. Conch. iii.p. 452. pl.99, f. 13-15. D.F.

$1894 \quad, \quad$ " $\quad$ Ancey, Mém. Soc. Zool. Fr. vii. p. 218. $D$.

1899 " glutinosa, Pfr. (= petersi, von Mts.), Smith, P.Z.S. p. 589. N.

$1904 \quad$ " $\quad$, $\quad$ Pilsb., Man. of Conch. xvii. p. 61.pl. 9, f. 23, 24. D.F.

Type in British Museum.

Hab. Lorenzo Marques. Tette (petersi, Peters).

A. glutinosa was described as from "West Africa."

379. Achatina granulata, Pfeiffer.

1852 Achatina gramulata, Pfr., P.Z.S. p. 66. D.

1853

1861

,

1868

1870

1889
„, Mon. Hel. iii. p. 484. D. semigranosa, Pfr., P.Z.S. p. $25 . \quad D$.

" Mal. Blätt. viii. p. 78. D.

" Mon. Hel. vi. p. $216 . \quad D$.

granulata, Pfr., Semp., Reis. im Arch. Philippin. ii, 3. p. 143. pl. 12, f. 2. pl. 16, f. 14. A.R. , (=zebra, var. granulata, Krs.), von Mts., Sitz.-Ber.Ges. Nat. Fr. Berlin, p. 164. 
1890 Achatina granulata, Pfr. (= semigranosa, Pfr.), Smith,A.M.N.H. vi. p. 391. $N$.

1897 „, drakensbergensis, M. \& P., A.M.N.H. xix.p. 636. pl. 17, f. 7. D.F.

1904, 5 Cochlitoma

All types in British Museum.

Hab. Natal (coll. Cuming). Karkloof (McBean). Cape Natal (semigranosa, Plant). Inhluzani (drakensbergensis); Durban(Bowker). All along the coast from Port Shepstone to Tugela and beyond into Zululand, and at various inland localities as far as Van Reenen's Pass; common at Pietermaritzburg (Burnup and Quekett).

Cape of Good Hope. Pondoland (Beyrich).

\section{Achatina greyi, da Costa.}

1907 Achatina greyi, da Costa, Proc. Mal. Soc. vii. p.226. pl.20, f.1. D.F.

\begin{tabular}{|c|c|c|c|c|c|}
\hline & $"$ & ovata & $"$ & $"$ & p. 226 . pl.20, f.2. D.F. \\
\hline & $"$ & zebrina & $"$ & ", & f.5. 1 \\
\hline & , & subovata & ", & " & p.227.pl.20, f.4. D.F. \\
\hline
\end{tabular}

Types in British Museum.

Hab. Rhodesia. Salisbury (Miss Weineck).

Described from the South Congo.

Judging from the types, the foregoing names all refer to forms of one variable species. Examples from Salisbury combine the attributes of two or more of the forms, but are not specifically distinct.

381. Achatina imiculata, Lamarck.

[S.A.M.]

1821 Helix (Cochlitoma) immaculata, Lam., Fér., Tabl. Syst. Moll. pt. 3. p. 73 (or 69).

1822 Achatina immaculata, Lam., Hist. nat.An.s. Vert. vi, 2.p.128. D.

1830

1838

1848

"

1851

1859

1879

1899
Desh., Encycl. Méth. Vers. ii.p.9. D. , Hist. nat. An. s. Vert. viii. p. $295 . \quad D$.

" Krs., Südafr. Moll. p. 81. N.D.

" Pfr., Mon. Hel. ii. p. 251. D.

" Desh., Hist. Nat. Moll. ii, 2. p. 158. pl. 127, f. 1, 2. D.F.

„ Pfr., Mon. Hel. iv. p. 600, D.

", Gibbons,Journ.ofConch.ii.p.143. N.

", Smith, Proc. Mal. Soc. iii. p. 309

(Epiphragm.) 
1899 Achatina immaculata, Lam., Smith, P.Z.S. p. 589. N.

$1904-5$

1907
„ Pilsb., Man. of Conch. xvii. p. 50. pl. 11 , f. 35 . pl. 43, f. 50 . D.F. Melv. \& Standen, Manchester Memoirs, li, 4. p. 12 . N.

Type in Geneva Museum.

Hab. Unknown to Lamarck or Férussac.

Transvaal. Barberton (Cregoe). Pietersburg District (Hewson). Messina (Gordon). Pietpotgietersrust District (Money).

Rhodesia. Bulawayo; Zimbabwe (in coll. Crawford).

Lorenzo Marques. Delagoa Bay (fide Pfeiffer). Inhambane (Gibbons).

Natal (Wahlberg). Tugela Valley (Quekett). Durban (in British Museum).

Zululand (in British Museum).

Widely distributed as far north as Nyassaland.

E. A. Smith (P.Z.S., 1899, p. 589) remarks, "A. layardi, Pfr." (Mal. Blätt. v. 1858, p. 238. D.), “ is a variety of this species, rather more profusely spotted than the type."

\section{Achatina indotata, Reeve.}

1849 Achatina indotata, Rve., Conch. Icon. pl. 6, f. 18. D.F.

1904 Cochlitoma indotata , Pilsb., Man. of Conch. xvii.p. 83.pl. 6, f. 10 . D.F.

Type in British Museum.

Hab. Cape of Good Hope. Elim (in coll. Albers, fide von Martens).

Described as from West Africa (coll. Cuming).

383. Achatina Jacobi, da Costa.

1906 Achatina jacobi, da Costa, Proc. Mal. Soc. vii. p. 11. D.F.

Type in British Museum.

Hab. Mashonaland. Rusape (Morrell).

384. Achatina lintere, Sowerby.

1889 Achatina lintere, Sow., P.Z.S. p. 580. pl. 56, f. 11 . D.F. 1904, 5 Cochlitoma lintere, Sow., Pilsb., Man. of Conch. xvii. p. 102. pl. 29, f. 43. D.It.

Type in Exeter Museum. 
Hab. CAPe of Good Hope. Port Elizabeth, "in drift sand, extinct" (Crawford).

385. Achatina imingstonei, Melv. \& Pons. [S.A.M.] 1897 Achatina livingstonei, M.\&P., A.M.N.H.xix.p.636. pl.17, f.6. D.F. 1904 Cochlitome ," „ Pilsb., Man. of Conch. xvii. p. 104. pl. 14, f. 1. D.F.

Type in British Museum.

Hab. British Bechuanaland. Kuruman (Livingstone).

Cape of Good Hope. Prieska (Gibbons). Buchu Berg, Hay District (Gould).

386. Achatina machachensis, Smith.

1902 Achatina machachensis, Smith, Proc. Mal. Soc. v. p. 169. D.F. 1904, 5 Cochlitoma " „ Pilsb., Man. of Conch. xvii.

Type in British Museum. p. 84. pl. 41, f. 7. D.F.

Hab. Basutoland. Mount Machacha (Crawshay).

387. Achatina natalensis, Pfeiffer.

1854 Achatina natalensis, Pfr., P.Z.S. p. 294. D.

$1859 \quad$ ",$\quad$ ", Mon. Hel. iv. p. 602. D.

1904 Cochlitoma ." $\quad$ " Pilsb., Man. of Conch. xvii.p. 102. D.

Type in British Museum.

Hab. Natal. Port Natal (Plant).

Cape of Good Hope. Somerset East (Miss Bowker).

TransvaAl. Wakkerstroom (Bowker).

Orange Free State. Vredefort Road (Barrett Hamilton).

Lorenzo Marques. Delagoa Bay (Plant).

388. Achatina edigtra, Melv, \& Pons.

1894 Achatina oedigyra, M. \& P., A.M.N.H. xiv. p. 92. pl. 1, f. 7. D.F. 1904, 5 Cochlitoma adigyra, M. \& P., Pilsb., Man. of Conch. xvii. p. 94. pl. 26, f. 29 . D.F.

Type in British Museum.

Hab. Cape of Good Hope. Craigie Burn, Somerset East (Mrs. Barber).

Rhodesia. Salisbury (in British Museum).

389. Achatina panthera (Férussac).

1821 Helix (Cochlitoma) panthera, Fér., Tabl.Syst. Moll.pt.3.p.53(or49). 1838 Achatina panthera, Fér., Desh., Hist, nat.An.s. Vert.viii.p.309. D. 
1846 Achatina lamarckiana, Pfr., P.Z.S. p. 115. D.

\begin{tabular}{|c|c|c|c|}
\hline $\begin{array}{l}1847 \\
1848\end{array}$ & ," & ", & $\begin{array}{l}\text { " A.M.N.H. xix. p. 269. D. } \\
" \text { Mon. Hel. ii. p. } 253 . \quad D .\end{array}$ \\
\hline & & panthera, & Fér., Pfr., Mon. Hel. ii. p. 252。 D. \\
\hline & 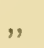 & ," & " Rve., Conch. Icon. pl. 3, f. 12. D.F. \\
\hline & ", & , & $\begin{array}{l}\text { " Desh., Hist. Nat. Moll. ii, 2. p. } 159 . \\
\text { pl. } 126, \text { f. 1, 2. pl. 132, f. 1, 2. D.F. }\end{array}$ \\
\hline 60,63 & , & $"$ & "Pfr., Conch.Cab.p. 327.pl.28, f. 1. D.F. \\
\hline 79 & $"$ & ," & Pfr., Gibbons, Journ. of Conch. ii. p. 143. 1 \\
\hline & ", & ", & Fér., Bgt., Moll. de l'Égypte, p. 9. N. \\
\hline 09 & $"$ & ", & $\begin{array}{l}\text { von Mits., Sitz.-Ber. Ges. Nat. Fr. } \\
\text { Berlin, p. 164. N. }\end{array}$ \\
\hline & ", & ", & " von Mts., ibid.p. $86 . \quad N$. \\
\hline & ", & , & " Ancey, Mém. Soc. Zool.Fr.vii.p.219. D. \\
\hline & ", & ," & " von Mts., D.-O.-Afr. p. 83. $N$. \\
\hline & ", & ," & " Smith, P.Z.S. p. 589. pl. 34, f.1. N.F \\
\hline & " & ," & $\begin{array}{c}\text { var. minor, Fér., Junod, Bull. S } \\
\text { Vaudoise, xxxv. p. 278. N. }\end{array}$ \\
\hline 04,5 & ", & , & $\begin{array}{l}\text { " Pilsb., Man. of Conch. xvii. p. } 41 . \\
\text { pl. } 38, \text { f. 30, 31. pl. 39, f. 32. pl. } 62 \text {, } \\
\text { f. 22-24. pl. 63, f. 30, 32. D.F.A.R. }\end{array}$ \\
\hline
\end{tabular}

Hab. Unknown to Férussac.

Transvaal. Queensriver, near the Victoria Mine, Barberton District (Schenck). Lebombo Mountains, between Barberton and Delagoa; Kapaira (Beyrich).

Lorenzo Marques. Inhambane (Gibbons; Bowker). Tette (Peters; Kirk). Rikatla (Junod).

Rнореsia. Sebakwe (Dodds).

Widely distributed through East and Central Africa and the islands of the Indian Ocean. A. lamarckiana was described from Madagascar.

In the South African Museum are two shells of this species, recorded as from "Port Elizabeth" (Fairbridge). If the locality is correct, the specimens were almost certainly introduced.

Pilsbry (1904) includes A. mossambica, Brancsik, and A. lechaptoisi, Ancey, in the synonymy.

390. Achatina parthenia, Melv. \& Pons.

1903 Achatina parthenia, M.\&P., A.M.N.H. xii.p.605.pl.32,f.10. D.F. 1904 Cochlitoma ", " Pilsb., Man, of Conch. xvii. p. 100. pl. 12, f. 38. D.F.

Type in British Museum. 
Hab. Zululand. Lower Umfolosi Drift; Makowe (Burnup). Ubomba (Toppin).

391. Achatina PASSARgei, von Martens.

1900 Acluatina passargei, von Mts., Sitz.-Ber. Ges. Nat. Fr. Berlin, p. 119. $D$.

1904

Die Kalahari, pp. 754,755 (f. 1). D.F.

Type in Zool. Mus. Berlin.

Pilsb.,Man.ofConch.xvii.p.70, D.

Hab. Ovampoland. Sodanna (Passarge).

392. Achatina penestes, Melv. \& Pons.

1893 Achatina penestes, M.\& P., A.M.N.H. xii. p. 104. pl. 3, f. 3. D.F. 1904, 5 Cochlitoma penestes, M. \& P., Pilsb., Man. of Conch. xvii. p. 100. pl. 28, f. 40. D.F.

Type in British Museum.

Hab. Transvaal. Pretoria (ex coll. Wotton)

393. Achatina Pentheri, Sturany.

1898 Achatina pentheri, Stur., S.A. Moll. p. 56. pl. 2, f. 40. D.F.

1904, 5 Cochlitoma pentheri, Stur., Pilsb., Man. of Conch. xvii. p. 81. pl. 30 , f. 50. D.F.

Type in Naturh. Hofmus. Vienna.

Hab. Natal. Durban (Penther).

394. Achatina rhabdota, Melv. \& Pons.

1898 Achatina rhabdota, M. \& P., A.M.N.H. i. p. 29. pl. 8, f. 11. D.F. 1902

Smith, Proc. Mal. Soc.v. p. 169. N

1904, 5 Cochlitoma rhabdota, M. \& P., Pilsb., Man. of Conch. xvii. p. 91. pl. 27, f. 37. D.F.

Type in British Museum.

Hab. "South Africa" (fide M. \& P.).

Littile Namaland. Port Nolloth (fide Watson).

395. Achatina scevola, Melv. \& Pons.

1893 Achatina scavola, M. \& P., A.M.N.H. xii. p. 104. pl. 3, f. 2. D.F. 1904, 5 Cochlitoma scavola, M. \& P., Pilsb., Man. of Conch. xvii. p. 98. pl. 34, f. 11 . D.F.

Type in British Museum.

Hab. Northern Transvad. Zoutpansberg (Bowker). 
396. Achatina schencki, von Martens.

1889 Achatina schencli, von Mts., Sitz.-Ber. Ges. Nat. Fr. Berlin, 1). 164 . I).

1894

1899 Conch. Mitth. iii, 3. p. 8. D. Gude, Journ. of Malac. vii. p. 90. (Err.typ.)

1904 Cochlitoma schencki ", Pilsb., Man. of Conch. xvii. p. 96. pl. 5, f. 1. D.F.

Type in Zool. Mus. Berlin.

Hab. Transvaal. "Macmac, near Lyderburg" (Schenck, 1886). Noте.--Macmac is in the Barberton District.

\section{Achatina schinziana, Mousson.}

1887 Achatina schinziana, Mouss., J.de C.xxxy. p. 294. pl. 12, f. 3. D.F. 1889

1904 Morel., J. de C. xxxvii. p. 9. N. Pilsb., Man. of Conch. xvii. p. 16. pl. 17, f. 19. I).F.

Type in Zurich Museum.

Hab. Ovampoland. Ondonga (Schinz). Sodanna (Passarge).

? Lorenzo Marques. Rikatla (Junod).

NoтE.-Considerable doubt attaches to more than one of Junod's localities, as it appear's almost certain that some of the species mentioned in his article on Delagoa Bay were wrongly identified.

var. DEgenerata, Böttger.

1910 Achatina schinziana, Mouss., var. degenerata, Bttg., Abh. Senckenb. Naturf. Ges. Frankfurt, xxxii. p. 447. pl. 28, f. 12 . D.F.

Type in Senckenberg Museum, Frankfurt.

Hab. Bechuanaland. Kakir (Schultze).

398. Achatina semidecussata, Menke.

1846 Achatina semidecussata, Mke., Pfr., Symb. iii. p. 91. D.

1847

1848

1860,63

1889
"Phil., Abb. u. Beschr. ii. p. 213. pl. 16,1, f. 1 . D.F.

" Krs., Südafr. Moll. p. 81 . N.

, Pfr., Mon. Hel. ii. p. $257 . \quad$ D.

" " Conch. Cab. p. 336. pl. 2, f. $2,3 . \quad$ D. $F$.

"von Mts., Sitz.-Ber. Ges. Nat. Fr. Berlin, p. 164. L. 
Reference List of South African Non-marine NTollusca. 201

1904, 5 Cochlitoma semidecussata, "Mke." Pfr., Pilsb., Man. of Conch. xvii. p. 80. pl. 30, f. 51-53. D.F.

Type in Stettin Museum.

Hab. Natal (Menke). Durban (Penther). Tongaat (Burnup). Malvern (Miss Bowker).

Transvaal. Upper Olifant River, north of Middelburg (fide von Martens).

$$
\text { 399. Achatina simplex, Smith. }
$$

1878 Achatina simplex, Smith, Quart. Journ. of Conch. i. p. $350 . \quad D$. 1904 Cochlitoma ", " Pilsb., Man. of Conch. xvii. p. 98. pl. 12, f. 37. D.F.

Type in British Museum.

Hab. Natal. Port Natal (Sutherland, 1860). Tugela Valley (fide Quekett). Ladysmith (Burnup).

Transvaal. Between Delagoa Bay and Lydenburg (Wilms, fide von Martens).

400. Achatina smithi, Craven.

1880 Achatina smithii, Crvn., P.Z.S. p. 617. pl. 57, f. 1. D.F.

$1889 \quad$ " $\quad$ " , Morel., J. de C. xxxvii. p. 9. N.

$1898 \quad$ " $\quad$ " $\quad$, Stur., S.A. Moll. p. 57. N.

1904 Cochlitoma ", $\quad$ " Pilsb., Man. of Conch. xvii. p. 91. pl. 11, f. 36 . D.F.

Original in British Museum.

Hab. Transvaal. Lydenburg (Craven).

Matebililand. Maitengue River (Penther, fide Sturany).

401. Achatina subcylindrica, Preston.

1909 Achatina subcylindrica, Prest., A.M.N.H.iii. p. 182 pl.7,f.8. D.F.

Type in coll. Putzeys.

Hab. Natal (fide Preston).

402. Achatina transvaduensis, Smith.

1878 Achatina transvaalensis, Smith,Quart.Journ.of Conch.i.p.351. D. 1904 Cochlitoma ", " Pilsb.,Man.ofConch.xvii.p.99. D.

Type in British Museum.

Hab. Transvaal. Lydenburg (in British Museum; Craven).

403. Achatina ustulata, Lamarck.

1821 Helix (Cochlitoma) ustulata, Lam., Fér., Tabl. Syst. Moll. pt. 3. p. 74 (or 70). 
1822 Achatina ustulata, Lam., Hist. nat. An. s. Vert. vi, 2.p. 130. D.

1838

1842

1848

"

1849

1851

1890
Desh., Hist. nat. An. s. Vert. viii. p. 297. $D$.

Rve., Conch. Syst.ii.p.86.pl.177,f.5. F.

Krs., Südafr. Moll. p. $81 . \quad N$.

Pfr., Mon. Hel, ii. p. 257. D.

" Rve., Conch. Icon. pl. 12, f. 40. D.F.

" Desh., Hist. Nat. Moll. ii, 2. p. 164. pl. 125, f. 1, 2. D.F.

" von Mts., Sitz.-Ber. Ges. Nat. Fr. Berlin, p. $88 . \quad N$.

1904, 5 Cochlitoma ustulata, Lam., Pilsb., Man. of Conch, xvii. p. 89. pl. 28, f. 38. pl. 29, f. 45 . D.F.

Type in Geneva Museum.

Hab. Unknown to Lamarck or Férussac.

Cape of Good Hope. George District (Krauss). Knysna (Farquhar; Crawford). Pondoland (Beyrich).

Natal. Durban (Penther, fide Sturany).

\section{Achatina varicosa, Pfeiffer.}

1861 Achatina varicosa, Pfr., Mal. Blätt. viii. p. 73. pl. 2, f. 7, 8. D.F. $1868 \quad$ " , Mon. Hel. vi. p. 215. D.

$1869 \quad$ " $\quad$, Novit.Conch.iii.p.490.pl.106,f.1,2. D.F 1904, 5 Cochlitoma varicosa, Pfr., Pilsb., Man. of Conch. xvii. p. 92. pl. 26, f. 30-33. D.F.

Type in Stettin Museum.

Hab. Cape of Good Hope. Enon, north of Port Elizabeth (Hartvig). Grahamstown (Farquhar).

405. Achatina vestita, Pfeiffer.

[S.A.M.]

1854 Achatina vestita, Pfr., P.Z.S. p. 293. D.

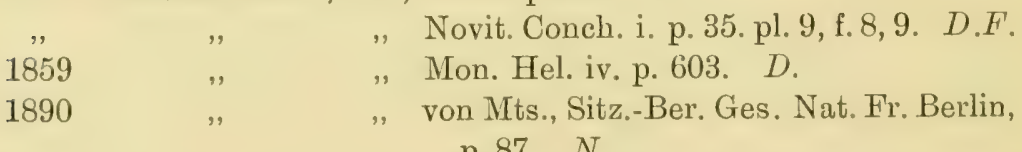

1904, 5 Cochtitoma vestita, Pfr., Pilsb., Man. of Conch. xvii. p. 81. pl. 30, f. 46-49. D.F.

Type in British Museum.

Hab. Natal. Cape Natal (Plant).

Cape of Good Hope. Pondoland (Beyrich). Port St. John's (Shortridge). 
Zululand. Kosi Bay (Toppin).

Lorenzo Marques. Delagoa Bay (Plant).

406. Achatina zebra (Chemnitz).

1767 Bulla achatina, var.livida, Lin., Syst. Nat., Ed.12.i, 2.p.1186. D. $1780 \quad, \quad$ " "Lin.," Born, Test. Mus. Caes. Vind. p. 208. pl. 10, f. 1. D.F.

1785 " " var. livida, Lin., Sparrman, A Voyage to the Cape of Good Hope, ii. p. 347. N.

1786 ", zebra, Chem., Conch. Cab. ix, 2.p. 22. pl. 118, f. 1014. D.F. $1788 \quad$ „ " Müll., Gmel., Syst. Nat., Ed. 13. 1, 6. p. 3431. D. 1789 Bulimus zebra, Brug., Enc. Méth. Vers, i. p. 357. D.

1797 Chersina " Humph., Mus. Calonn. p. 63. N.

1810 Achatinus zebra, de Roissy, de Montf., Conch. Syst. ii. p. 419. pl. 105. D.F.

1817 Bulla achatina, Lin. (var. 4), Dillw., Descr. Cat. i. p. 495. N. 1822 Achatina zebra, Lam., Hist. nat. An. s. Vert. vi, 2. p. 128. D. 1837 " borniana, Beck, Index Moll. p. 75. (Emend. Nom.)

$1838 \quad " \quad z e b r a$, Lam., Desh., Hist. nat. An.s. Vert. viii. p. 29j. D. 1840 „ " Chem., Küst., Conch. Cab. pl. 2, f. 3. F.

1842 " chemnitziana, Pfr., Symb. ii. p. 132. (Emend.) " $\quad$ " zebra, Chem., Rve., Conch. Syst.ii.p. 88.pl.179, f.17. F. $1848 \quad " \quad " \quad$ (=chemnitziana), Pfr., Mon. Hel. ii. p. 250 . $D$.

" Lam., Krs., Südafr. Moll. p. 80. D.

$1849 \quad "$,

1851

" Chem., Rve., Conch. Icon. pl. 7, f. 23. D.F.

" Lam., Desh., Hist. Nat. Moll. ii, 2. p. 156. pl. 133. D.F.

1853

1857

" Chem., Pfr., Mon. Hel. iii. p. 482. Varr.

1860

" Conch. Cab. p. 291. pl. 23 (1853), f. $1 . D . F$.

(cum var. borniana, Beck), von Mts., Die Helic. pp. 203, 204. D.N.

" $\quad$ " capensis, Alb., von Mts., Die Helic. p. 203. N.

1868 " zebra, Chem., var. borniana, Beck, Pfr., Mon. Hel. vi. p. 212. $D$.

$1870 \quad " \quad$ " $\quad$ "Semp., Reis. im Arch. Philippin. ii, 3.

1890 p. 144. pl. 12, 1, 22. Enbryo.

$1890 \quad " \quad " \quad$ " Smith, A.M.N.H. vi.p. 392. N.

$1898 \quad$ " " $\quad$ Brug., M. \& P., Proc. Mal. Soc. iii. p. 179.

1902 " $\quad$ Chem., Smith, Proc. Mal. Soc. v. p. 169. N. 
1904, 5 Cochlitoma zebra, Brug., Pilsb., Man. of Conch. xvii. p. 85. pl. 28, f. 39. pl. 64, f. 67. D.F. \& Embryo.

Type-ubi?

Hab. Cape of Good Hope (capensis, in coll. Albers). Sitsicamma (Sparrman). George and Uitenhage Districts (Krauss). Caffraria (Delalande; bomiana, fide Beck). Port Elizabeth; Alexandria; Mossel Bay; Grahamstown (Crawford). Lovedale; Pirie Forest; East London (Godfrey). Alicedale (Pillens). Toise River, Cathcart (Mrs. Gore). Stutterheim (French). Knysna (Purcell).

Cape Peninsula. Camps Bay (Dale; Morris).

var. Fulgurata, Pfeiffer.

1851 Achatina fulgurata, Pfr., P.Z.S. p. 258. D.
1853 " ", Mon. Hel, iii. p. 486 . D.
$1854 \quad$ " $\quad$ " A.M.N.H. xiii. p. $147 . \quad$ D.
1860, $63 \quad$ ", $\quad$ "Conch. Cab. p. 319. pl. 26, f. 1. D.F. 1904, 5 Cochlitoma fulgurata, Pfr., Pilsb., Man. of Conch. xvii.p. 85. pl. 27, f. 34. D.F.

Type in British Museum.

Hab. Cape of Good Hope. Somerset East (Miss Bowker). Grahamstown; Kowie (Farquhar).

Described as from "West Africa" (coll. Cuming).

var. GRANULATA, Krauss.

1848 Achatina zebra, Lam., var.granulata, Krs., Südafr. Moll.p. 80. D. 1904, 5 Cochlitoma granulata, Krs. (=granulata, Pfr.), Pilsb., Man. of Conch. xvii. p. 79. pl. 29, f, 44 . D.F.

Type in Stuttgart Museum.

Hab. Cape of Good Hope. Outeniqua, George District (Krauss). Natal (Wahlberg, fide Krauss).

var. KRAUSSI, Reeve.

1842 Achatina kransii, Rve., P.Z.S. p. 55. D.

\begin{tabular}{|c|c|c|c|c|}
\hline & 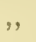 & ", & ", & Conch. Syst. ii. p. 88. pl. 179, f. 19. I \\
\hline 1848 & " & kraussii & ", & Pfr., Mon. Hel. ii. p. 250. $D$. \\
\hline " & ", & ", & ," & Krs., Südafr. Moll. p. 81. D.N. \\
\hline 1849 & ," & kraussi & , & Conch. Icon. pl. 6, f. 21 . D.F. \\
\hline 1860 & ", & $"$ & , & $\begin{array}{l}\text { Pfr., Conch. Cab. p. 329. pl. } 23 \text { (1853), } \\
\text { f. 2. D.F. }\end{array}$ \\
\hline 1890 & ," & ", & ," & $\begin{array}{l}\text { von Mts., Sitz.-Ber. Ges. Nat. } \\
\text { Berlin, p. } 87 . \quad N .\end{array}$ \\
\hline 898 & ", & " & $"$ & Stur., S.A. Moll. p. 55. N. \\
\hline
\end{tabular}


Reference List of South African Non-marine Mollusca. 205

1898 Achatina zebra, Brug., var. kraussi, Rve., M. \& P., Proc. Mal.

Soc. iii. p. 179.

1904, 5 Cochlitoma kraussi, Rve., Pilsb., Man. of Conch. xvii. p. 87. pl. 16, f. 10, 11. pl. 27, f. 36 . D.F.

Type in British Museum.

Hab. Cape of Good Hope. Coega River, Algoa Bay (Krauss).

Reeve's original name and loc., Cape Natal (Krans, in coll. Cuming), are incorrect.

Crawford, in locating this shell from Coega (loc. sol.), writes: "I consider this a species, and have not seen it from anywhere else. The form is different from $A$. zebra, and can be intimated from colourless shells."

var. MINOR, Pfeiffer.

1853 Achatina zebra, Chem., var. minor, Pfr., Mon. Hel. iii. p. 482 . N. 1857

, " " , Conch.Cab.p.292.pl.23 (1853), f. 1. N.F.

Hab. "Madagascar and Cape" (Krauss).

var. OBEsA, Pfeiffer.

?1851 Helix zebra, Fér., Desh., Hist. Nat. Moll. pl. 133, middle figure. 1854 Achatina obesa, Pfr., Mal. Blätt. i. p. 224. D.

$1859 \quad$, " Mon. Hel, iv. p. 600. D.

$1890 \quad$ " " " "probably only a stunted form of zebra," Smith, A.M.N.H. vi. p. 393. N.

1904, 5 Cochlitoma zebra, Fér., var. obesa, Pfr., Pilsb., Man. of Conch. xvii. p. 87. pl. 27, f. 35 . D.F.

Type in Stettin Museum.

Hab. Cape of Good Hope. Port Elizabeth District (Crawford).

Originally described as from West Africa.

407. Achatina zebrula, von Martens.

1900 Achatina zebrula, von Mts.,Sitz.-Ber.Ges.Nat.Fr.Berlin,p.118. D.

1904 Cochlitoma ," , Pilsb., Man. of Conch. xvii. p.90. D.

Type in Zool. Mus. Berlin.

Hab. Transvaal. Between Delagoa and Lydenburg (Wilms).

Genus CÆCILIOIDES, Férussac, 1817.

(de Blainv., Dict. Sci. Nat. v. Suppl. p. 129, as Ceclionides, and vii. p. 332, as

Cécilioide, emend. Herrmannsen, 1846, Indicis Generum Malac. i p. 150)

(=Cacilionella, Bgt., 1856.) 


$$
\begin{aligned}
& \text { Type of Genus, C. acicula (Müll.). } \\
& \text { 408. C五Cilioides acicula (Müller). }
\end{aligned}
$$

1774 Buccinum acicula, Müll., Verm. ii. p. 150. D.

1798 ," longiusculum, Adams, Essays on the Microscope, p. 630. pl. 14, f. 26. D.F.

1801 „, obtusulum, Turt., Syst. Nat.(English Ed.), iv.p.416. D. 1803 ", terrestre, Mont., Test. Brit. i. p. 248. pl. 8, f. 3. D.F. 1805 Bulimus acicula, Müll., Drap., Hist. Moll. Fr.p. 75. pl.4,f.25. D.F. 1821 " „ C. Pfr., Syst. Anordn. deutsch. L.-u. W.-Schn. p. 51. pl. 3, f. 8, 9. D.F.

1826 Achatina eburnea, Risso, Hist. Nat. Eur. mérid. iv. p. 81. D. 1827 " alba, Brown, Illustr. Brit. Conch. pl. 41, f. 80. F. 1828 " acicula, Mill., Flem., Hist. Brit. An. p. 267. D. 1831 " $\quad$ " Lam., Turton, Manual, p. 89. D.

1832 Columna miliaris, de Christ. \& Jan, Cat. Mantissa, p. 2. D. 1833 Cionella acicula, Müll., Jeffr., Linn. Trans. xvi. p. 348. D.

1836 Achatina pusilla, Scacchi, Cat. Conch. Reg. Neapol. p. 16.

1847 Acicula pellucida, Leach in Gray, A.M.N.H. xx. p. 269.

1848 Achatina acicula, Müll., Pfr., Mon. Hel. ii. p. 274. D. 1856 Cacilionella acicula, Müll., Bgt., Rev. et Mag. Zool. p. 382. D.

, " $\quad$ " $\quad$ " Amén. malac. i. p. 215. pl. 18, f. 1-3. D.F.

1862 Achatina acicula, Müll., Jeffr., Brit. Conch.i. p. 297.pl. 7, f. 1821, and v (1869). pl. 18, f. 3. D.F.

1863 Pfr., Conch. Cab. p. 352. pl. 29 (1865), f. 12,13 . D.F.

1908 Cacilioides acicula „, Pilsb., Man. of Conch. xx. p. 9. pl. 1, f. 1-10, 13, 14, 16-18. D.F.

Original of acicula in University Zool. Mus. Copenhagen.

Hab. Cape of Good Hope. Cradock (in coll. Ponsonby). Wynberg (Connolly). Prieska (Gibbons; Gould).

Griqualand West. Kimberley (Miss Wilman).

Transvaal. Pietpotgietersrust (Connolly).

Orange Free State. Bloemfontein (Connolly).

409. Cæecilioides advena (Ancey).

1888 Cacilianella advena, Ancey, Le Naturaliste, x. p. 215. D. 1908 Cacilioides , " Pilsb., Man. of Conch. xx. p. 35. D.

Type-ubi?

Hab. Ovampoldnd. Disappointment Vlei (Andersson \& Chapman). 
Ancey's locality, "Disappointment Key," is probably a slip. On labels in the Layard collection the spelling is Disappointment Vlei, which for many reasons seems the more likely name.

\section{Cacilioides goktweanus (Böttger).}

1870 Cionella gokweana, Bttg., Ber. Offenbach. Ver. f. Naturk. xi. p. 47. pl. 1, f. 2. D.F.

1910

Abh. Senckenb. Naturf. Ges. Frankfurt, xxxii. p. $449 . \quad N$.

Type in Senckenberg Museum, Frankfurt.

Hab. Bechuanaland. Sub-fossil at the Gokwe River, near $22^{\circ}$ S. lat. and $28^{\circ} \mathrm{E}$. long. (Hübner).

Transvaal. Pienaars Poort (Connolly).

\section{Cacilioides ovampoensis (Melv. \& Pons.).}

1892 Cionella ovampoensis, M. \& P., A.M.N.H. ix. p. 91. pl.6, f.1. D.F. 1898 " (Cacitionella) ovampoensis, M.\& P., Stur., S.A. Moll. p. 62 . $N$.

1908 Cecilioides ovampoensis, M. \& P., Pilsb., Man. of Conch. xx. p. 36. pl. 3, f. 52 . D.F.

1910 Cacilianella ovampoensis, M. \& P., Bttg., Abh. Senckenb. Naturf. Ges. Frankfurt, xxxii. p. 448. N.

Type in British Museum.

Hab. Ovampoland (in coll. Layard).

Lorenzo Marques. Matolla (Penther).

After examination with a strong lens, I am unable to find any specific difference between the type set of this species and examples from Pietpotgietersrust, which appear to be acicula (Müll.).

As advena, Ancey, and ovampoensis, M. \& P., were founded on shells gathered by the same collectors in the same locality, the two species are in all probability identical, but it has not been possible to compare the types.

Sub-Family STENOGYRIN A, Fischer, 1883.

(Man. de Conch. p. 486.)

Genus CURVELLA, Chaper, 1885.

(Bull. Soc. Zool. de France, x. pp. 48, 49.)

(=Hapalus, Albers, 1850, nec Billberg, 1820.)

Type of Genus, C, sulcata, Chaper. 
412. Curvella caloglypta, Melv. \& Pons.

1901 Curvella caloglypta, M. \& P., A.M.N.H. viii.p.320. pl.2, f.12. D.F. 1906 " Pilsb., Man. of Conch. xviii. p. 59. pl. 8, f. 22. D.F.

Type in British Museum.

Hab. Natal. Pietermaritzburg (Burnup).

413. Curvella catarracte (Melv. \& Pons.). [S.A.M.]
[S. 1837 Hapalus catarracte, M. \& P., A.M.N.H.xix.p.635.pl. 17, f.4. D.F .

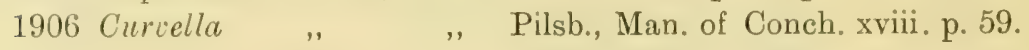
pl. 8, f. 23. D.F.

$1910 \quad$ " $\quad$, , Conn., A.M.N.H. vi. p. 268. N.

Type in British Museum.

Hab. Natal. Howick; Equeefa (Burnup). Durban (Penther). Cape of Good Hope. Pirie River (Godfrey).

414. Curvella croslyi, Burnup.

1905 Curvella croslyi, Bnp., Proc. Mal. Soc. vi. p. 302. pl. 16, f. 3, 4. D.F. $1906 \quad$," , Pilsb., Man. of Conch. xviii. p. 59. pl. 8, f. $27,28 . \quad D \cdot F$.

Type in British Museum.

Hab. Zululand. Makowe (Crosly).

415. Curvelua elevata, Burnup.

1905 Curvella elevata, Bnp., Proc. Mal. Soc. vi. p. 304. pl. 16, f. 10, 11. D.F.

1906 „, $\quad$ Pilsb., Man. of Conch. xviii.p. 60. pl. 8, f. 29,30 . D.F.

$1910 \quad$, Conn., A.M.N.H. vi. p. 269. N.

Type in British Museum.

Hab. Cape of Good Hope. Grahamstown (Farquhar).

416. Curvella globosa (Melv. \& Pons.).

1898 Hapalus globosus, M. \& P., A.M.N.H. ii. p. 128. pl. 7, f. 6. D.F. 1906 Curvella globosa ", Pilsb., Man. of Conch. xviii. p. 61. pl. 8, f. 31. D.F.

$1910 \quad$ " $\quad$ Conn., A.M.N.H. vi. p. 268. N.

Type in British Museum.

Hab. NataL. Stella Bush (Burnup).

CAPE of Good Hope. York Drakensberg, Griqualand East (Farquhar). 
417. Curvelua majubana, Connolly.

1910 Curvella majubana, Conn., A.M.N.H. vi. p. 270. pl. 6, f. 13. D.F. Type in British Museum.

Hab. NataL. Majuba (Connolly).

418. Curvella modesta, Connolly.

1910 Curvella modesta, Conn., A.M.N.H. vi.p. 271. pl. 6, f. 15. D.F. Type in British Museum.

Hab. CAPE OF Good Hope. Grahamstown; Cradock (Farquhar).

419. Curvelua saunderse, Connolly.

1910 Curvella saundersce, Conn., A.M.N.H. vi. p. 270. pl. 6, f. 14. D.F. Type in British Museum.

Hab. Zululand. Eshowe (Lady Saunders).

420. Curvella sinuosa, Melv. \& Pons.

1899 Curvella sinuosa, M. \& P., A.M.N.H. iv. p. 198. pl. 3, f. 12 . D.F. $1906 \quad$ ", Pilsb., Man. of Conch. xviii. p. 61.

Type in British Museum. pl. 8, f. 32. D.I.

Hab. NataL. Umkomaas (Burnup).

421. Curvelua straminea, Burnup.

[S.A.M.]

1905 Curvella straminea, Bnp.,Proc.Mal.Soc.vi.p.303.pl.16,f.5,6. D.F. $1906 \quad$ „, „ Pilsb., Man. of Conch. xviii. p. 62. pl. 8, f. 36,37 . D. $F$.

Type in British Museum.

Hab. Cape of Good Hope. Walmer (Miss Hickey).

422. Curvelia succinea, Burnup.

1905 Curvella succinea, Bnp., Proc. Mal. Soc. vi. p.303.pl.16,f.7,8. D.H'. 1906 "Pilsb., Man. of Conch. xviii. p. 62.pl.8, f. 38,39 . D.F.

Type in British Museum.

Hab. CAPE of Good Hope. Maeström Forest, Bedford (Farquhar).

Genus HYPOLYSIA, Melvill \& Ponsonby, 1901.

(A.M.N.H. viii. p. 318.)

Type of Genus, H. florentia, M. \& P. 
423. Hypolysia florentie, Melv. \& Pons.

1901 Hypolysia florentia, M. \& P., A.M.N.H.viii.p.318.pl.2,f.8 D.F.

1903

1906

,

"

1910

Type in British Museum.

\section{" $\quad$ " xii.p. 596. pl.32,f.13. $F$.}

" Pilsb., Man. of Conch. xviii. p. 37. pl. 10, f. 66,67 . D.F.

Conn., A.M.N.H. vi. p. 271. N.

Hab. Natal. Durban (Burnup).

Widely distributed over the South-Eastern province of the CAPE of Good Hope from Grahamstown, East London, and Port Elizabeth, through Natal to Eshowe in Zululand.

Transvaal. Rustenberg District (McBean).

A very variable form, which will probably be found to embrace more than one species when larger series are available for comparison.

Genus SUBULINA, Beck, 1837.

(Indęx Moll. p. 76, and Pilsb., 1906, Man. of Conch., xviii. p. 71.)

Type of Genus, S. octona (Brug.).

424. Subulina mamilata (Craven).

1880 Achatina mamillata, Crvn., P.Z.S. p. 215. pl. 22, f. 8. D.F.

1907 Opeas mamillata, Crvn., Dixey \& Longstaff, Trans. Entom. Soc. p. $361 . \quad L$.

Type in British Museum.

Hab. Rhodesia. Victoria Falls (Dixey \& Longstaff).

Deseribed from Nossi Bé, and also known from Magila.

Quite distinct from the next species, in whose synonymy Pilsbry has placed it.

425. Subuima octona (Bruguière).

1786 Helix octona Indice Occidentalis, Chem., Conch. Cab. ix, 2. p. 190. pl. 136, f. 1264. D.F.

1792 Bulimus octonus, Brug., Encycl. Méth. Vers, i. p. 325. D.

1817 Achatina crotallaria, Schumacher, Essai d'un nouv. Syst. Vers Test. p. 202. $D$.

1831 ", octona, Lam., Turton, Manual, p. 90. D.

1838 Bulime octonus, Brug., Desh., Hist. nat. An.s. Vert.viii.p. 233. D. 1839 Achatina novenaria, Anton, Ver'z. Conch. Samml. p. 44.

1842 " trochlea, Pfr., Symb. ii p. 59. D.

1849 " octona, Chem., Rve., Conch. Icon. pl, 17, f. 84 . D.F. $185 \pm$ Subulina octona, Chem., H.\&A.Ad.,Gen.rec.Moll.iii.pl.71,f.3a. $F$. 
1863 Achatina octona, Chem., Pfr., Conch. Cab. p. 342. pl. 37 (1865), f. 19, 20. D.F.

1868 Morel., Voy. Welwitsch, Moll. p. 80. pl. 6, f. 5. N.F.

1897 Subulina octona, Chem., von Mts., D.-O.-Afr. p. 123. N.

$1906 \quad$ ", Brug., Pilsb., Man. of Conch. xviii. pp. 73, 222. pl.12,f. 8,9,11,12.pl.39, f.28-37,39,40. D.F.

1907 Opeas octona, Chem., Dixey \& Longstaft, Trans. Entom. Soe. p. 361. L.

Type-ubi?

Hab. Rhodesia, Rain Forest, Victoria Falls (Dixey \& Longstatf).

An American species, introduced into nearly every part of the globe. Pilsbry's synonymy is chiefly followed above.

\section{Subulina vitrea, Mousson.}

1887 Stenogyra (Subulina) vitrea, Mouss., J. de C. xxxv. p. 296. pl. 12, f. 6. D.F.

1892 " $\quad$ chapmani, M. \& P., A.M.N.H. ix. p. 90. pl. 6, f. 3. D.F. 1906 Subulina vitrea, Mouss., Pilsb., Man. of Conch, xviii. p. 94. pl. 14, f. 50. D.F.

" $\quad$ " chapmani, M. \& P., Pilsb., ibid. p. 92. pl. 14, f. 46. D.F. 1910 ", vitrea, Mouss. (=chapmani, M. \& P.), Bttg., Abh.

Senckenb. Naturf. Ges. Frankfurt, xxxii. p. 148. N.

Type of chapmani in British Museum; vitrea in Zurich Museum.

Hab. Ovampoland (chapmani, Chapman). Ku-Ganab, southeast of Ondonga (vitrea, Schinz).

Judging from the figures and descriptions, Böttger seems to have good reason for uniting the above-mentioned species, which came from the same district.

Genus OPEAS, Albers, 1850.

(Die Helic. p. 175.)

Type of Genus, O. subula, Pfr. (gracile, Hutt.).

427. Opeas crawfordi (Melv. \& Pons.).

1893 Stenogyra crawfordi, M. \& P., A.M.N.H. xii. p. 105.pl. 3, f.4. D.F. 1906 Opeas crawfordi, M. \& P., Pilsb., Man. of Conch. xviii. p. 149. pl. 15, f. 74 . D.F. 
Type in British Museum.

Hab. Cape of Good Hope. Van Staaden's River (Crawford).

428. Opeas durbanense, Sturany.

1898 Opeas durbanense, Stur., S.A. Moll. p. 61. pl. 2, f. 42-44. D.F. 1906 " „ Pilsb., Man. of Conch. xviii. p. 149.

Type in Naturh. Hofmus. Vienna. pl. 15 , f. $75,76$. D.F.

Hab. Natal. Durban (Penther).

Founded on a single specimen.

\section{Opeas eulimoide (Preston).}

1909 Subulina eulimoides, Prest., A.M.N.H. iv. p. 499. D.F. 1910 Opeas eulimoide, Prest., Conn., A.M.N.H. vi. p. 267. N.

Type in British Museum.

Hab. Natal. Howick (Cregoe).

$$
\text { 430. Opeas lepidum, Connolly. }
$$

1910 Opeas lepictum, Conn., A.M.N.H. vi. p. 267. pl. 6, f. 12 . D.F.

Type in British Museum.

Hab. Cape of Good Hope. Fern Kloof, Grahamstown (Farquhar). Port Elizabeth (Crawford).

431. Opeas mcbeani, Melv. \& Pons.

1903 Opeas mebeani, M. \& P., A.M.N.H. xii. p. 604 pl. 31, f. 8. D.F. 1906 " „ Pilsb., Man. of Conch. xviii. p. 150. pl. 15, f. 77. D.F.

$1910 \quad$ ", $\quad$, Conn., A.M.N.H. vi. p. $266 . \quad N$.

Type in British Museum.

Hab. 'Transvaal. Boksburg (McBean, per Burnup). Hennop's River (Connolly). Middelburg (in coll. Ponsonby).

432. Opeas strigile (Melv. \& Pons.).

1901 Subulina strigilis, M. \& P., A.M.N.H. viii. p. 318. pl. 2, f. 7. D.F. 1906 Opeas strigilis, M. \& P., Pilsb., Man. of Conch. xviii. p. 150. pl. 15, f. 79. D.F'.

1910 ," strigile, M. \& P., Conn., A.M.N.H. vi. p. 266. N.

Type in British Museum.

Hab. Natal. Karkloof Bush (McBean). Dargle; Edendale; Pietermaritzburg; Enon Bush, Richmond; Hilton Road (Burnup). 
433. Opeas sublineare, Böttger.

[S.A.M.]

1910 Opeas sublinearis, Bttg., Abh. Senckenb. Naturf. Ges. Frankfurt, xxxii. p. 448. pl. 28, f. 14. D.F.

Type in Senckenberg Museum, Frankfurt.

Hab. Littue Namaland. Muishond (Schultze). Henkries (Lightfoot).

Griqualand West. Modder River (Miss Wilman).

434. Opeas tugelense (Melv. \& Pons.).

1897 Subulina tugelensis, M.\&P., A.M.N.H.xix. p. 637.pl.17, f. 9. D.F.

1906 Opeas tugelensis, M. \& P., Pilsb., Man. of Conch. xviii. p. 150. pl. 15, f. 78. D.F.

1910 " tugelense, M. \& P., Conn., A.M.N.H. vi. p. $266 . \quad$ N.

Type in British Museum.

Hab. Natal. Lower Tugela River; Tongaat; Pinetown; Pietermaritzburg (Burnup).

Lorenzo Marques. Delagoa Bay (Connolly).

Genus EUONYMA, Melv. \& Pons., 1896.

(A.M.N.H. xviii. p. 316, and Pilsb., 1906, Man. of Conch. xviii. p. 38.)

Type of Genus, Euonyma laocochlis, M. \& P.

435. Euonyua cacuminata (Melv. \& Pons.).

1892 Stenogyra cacuminata, M. \& P., A.M.N.H. ix. p. 85.pl.6, f. 2. D.F.

1906 Euonyma

Pilsb., Man. of Conch. xviii. p. 42. pl. 10, f. $71,73,74$. D.F.N.

1910

Type in British Museum.

Conn., A.M.N.H. vi. p. 259. N.

Hab. Cape of Good Hope. Bedford (Farquhar).

436. Eujonya crystallina (Melv. \& Pons.). [S.A.M.]
[S. 1896 Subulina crystallina, M.\&P.,A.M.N.H.xviii.p.316.pl.16,f.4. D.F.

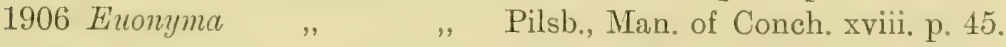
pl. 10, f. 81 . D.F'.

1910 " " $\quad$ " Conn., A.M.N.H. vi. p. 255. N.

Type in British Museum.

Hab. Natal. Pietermaritzburg (Burnup). Widely distributed throughoui Natal.

Zululand. Eshowe (Lady Saunders).

Cape of Good Hope. King IVilliamstown (Godfrey). TVidely distributed in the Eastern Province. 
On account of its small size, it might be advisable to transfer the present species to Opeas, together with linearis, Krs., and pietersburgensis, Preston.

437. Euonyma gouldi, sp. nov., pl. 2, f. 7.

[S.A.M.]

Shell elongate, turriform, subrimate, olivaceous, thin, siightly glossy, semi-transparent. Spire produced, acute, apex bluntly rounded. Whorls 10, gradually increasing, becoming less convex as they grow in size; the first two smooth, remainder closely covered with faint, almost straight striæ, parallel to the slant of the outer lip. Suture well defined, but not deep. Aperture short, ovate, rounded at base. Peristome thin, simple. Outer lip curved outwards, slightly receding in an almost straight line to the base of the shell. Columella concave, margin very narrowly reflexed, forming a small rima. Shell $19.5 \times 5 \cdot 1$; apert. $4.3 \times 2 \cdot 2$; last whorl $7.1 \mathrm{~mm}$.

Type in South African Museum.

Hab. Cape of Good Hope. Prieska (Gould; van der Merve).

In all the specimens I have seen the sides of the spire are slightly convex about the sixth whor?.

Quite unlike any neighbouring species; pruizenensis, Conn., which resembles it in form, has curved, instead of straight, striation.

438. Euonyma Leocochlis (Melv. \& Pons.).

1896 Subulina leacochlis, M.\&P., A.M.N.H. xviii.p.316.pl.16,f.3. D.F. 1898 Euonyma lococochlis " Stur., S.A. Moll. p. 62. (Err. typ.) 1906 " leocochlis " Pilsb., Man. of Conch. xviii. p. 39.

Type in British Museum. pl. 10, f. 68. D.F.

Hab. CaPe of Good Hope. Humansdorp, St. Francis Bay (Fraser).

\section{Euonyma lanceolata (Pfeiffer).}

1854. Bulimus lanceolatus, Pfr., P.Z.S. p. 292. D.

1857 " micans, Pfr., Mal. Blätt. iv. p. 156. D.

$1859 \quad$ " " " " Mon. Hel. iv. p. 452. D.

" $\quad$ " lanceolatus, Pfr., Mon. Hel. iv. p. 455. D.

1906 Enconymu lunceolate, Pfr., Pilsb., Man. of Conch. xviii. p. 40. D.N. 1910 " Conn., A.M.N.H. vi.pp. 260,261. N.F.

Types in British Museum.

Hab. Natal. Cape Natal (Plant). Pietermaritzburg; Karkloof (Burnup). 
Zululand (in British Museum).

Lorenzo Marques. Delagoa Bay (in British Museum).

440. Euonyal Linearis (Krauss).

1848 Bulimus linearis, Krs., Südafr. Moll. p. 78. pl. 5, f. 3. D.F.

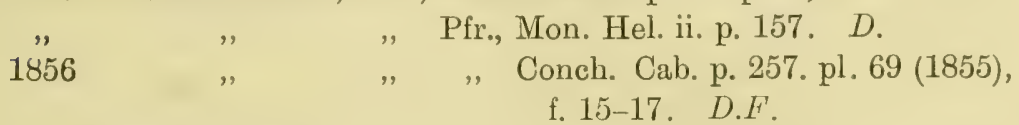

1898 Opeas lineare, Krs., Stur., S.A. Moll. p. 61. N.

1906 Euonyma linearis, Krs., Pilsb., Man. of Conch. xviii. p. 44. pl. 10 , f. 79,80 . D.F.

1910

Conn., A.M.N.H. vi.p. 252. pl.6, f. 4. D.N.F.

Type in Stuttgart Museum.

Hab. ? Transvadu. Mount Mohapaani, on the Limpopo (Wahlberg).

Not B. linearis, Rve., 1850, nor O. lineare, Stur., 1898.

Many different species have been attributed by various authorities to E. linearis. Morelet's loc., Port Elizabeth, is almost certainly wrong, while Reeve and Sturany appear to have regarded the then undescribed Hypolysia florentic, M. \& P., as typical of Krauss' species ; an error also followed by Preston (A.M.N.H. iv, 1909, p. 499). Craven's locs., Lydenburg, Transvaal, and Winburg, O.F.S., require further substantiation.

441. Euonyma hymnezformis (Melv. \& Pons.).

1901 Obeliscus lymneaformis, M.\&P.,A.M.N.H.viii.p.317.pl.2,f.5. D.F. 1906 Euonyma , Pilsb., Man. of Conch. xviii.p. 39. pl. 10, f. 69 . D.F.

$1910 \quad$," $\quad$ " $\quad$, Conn., A.M.N.H. vi. p. 260. N.

Type in British Museum.

Hab. Natal. Karkloof Bush (McBean).

Founded on two specimens; no more have occurred.

\section{Euonyma natalensis (Burnup).}

[S.A.M.]

1905 Obeliscus natalensis, Bnp.,Proc. Mal. Soc.vi.p.304.pl.16,f.9. D.F. 1906 Euonyma ,, , Pilsb., Man. of Conch. xviii.pp.41, 339. pl. 10, f. 72. pl. 31, f. 12, 13. D.N.F.

1910 , Conn., A.M.N.H. vi. p. 261. N.

Type in British Museum.

Hab. Natal. Umbogintwini ; Pietermaritzburg; Equeefa; Table Mountain (Burnup). 
443. Euonyma pieterisurgensis (Preston).

1909 Subulina pietersburgensis, Prest., A.M.N.H. iv. p. 499. D.F. 1910 Enonyma $\quad, \quad$ " Conn., A.M.N.H.vi.p.254. N. Type in British Museum.

Hab. Transvand. Pietersburg (fide Preston).

var. LEvis, Connolly.

1910 Enonyma pietersburgensis, Prest., var. levis, Conn., A.M.N.H. vi. p. 255. pl. 6, f. 8 . D.F.

Type in British Museum.

Hab. Transvan. Buis Kop; Pienaar's Poort; Pietpotgietersrust (Connolly). Zoutpansberg (Cregoe).

444. Euonyma platyacme, Melv. \& Pons.

1907 Fuonyma platyacme, M.\&P., A.M.N.H.xix. p. 101. pl.6, f.15. D.F. , Stenogyra beckeri, Fulton, A.M.N.H. xix. p. 154. pl. 10, f. 7. D.F. 1910 Euonyma platyacme, M. \& P. (=beckeri, Fulton), Conn., A.M.N.H. vi. p. $259 . \quad N$.

Both types in British Museum.

Hab. Cape of Good Hope. Kei Road Bush (Miss Hickey). Pondoland (beckeri, Becker). Hog's-back Mountain, Queenstown (Farquhar). Pirie Forest (Godfrey).

445. Euonyma pruizenensis, Connolly.

1910 Enonyma prizenensis, Conn., A.M.N.H. vi.p. 261.pl.6, f.11. D.F.

Type in British Museum.

Hab. Northern Transvalu. Pruizen; Pietpotgietersinut (Connoliy).

446. Euonyma purcelli (Melv. \& Pons.).

1901 Subulina purcelli, M. \& P., A.M.N.H. viii. p. 317. pl. 2, f. 6. D.F. 1906 Euonyma ", „ Pilsb., Man. of Conch. xviii. p. 42. pl. 10, f. $75 . \quad D . F$.

$1910 \quad$ " $\quad$ " , Conn., A.M.N.H. vi. p. 259. N.

Type in British Museum.

Hab. Cape of Good Hope. Houw Hoek, Caledon Division (Purcell; Lightfoot).

Described from an immature specimen; the adult shell attains a length of $25 \mathrm{~mm}$. 
447. Euonyma siliqua, Connolly.

1910 Euonyma siliqua, Conn., A.M.N.H. vi. p. 262. pl, 6, f. 10. D.F. Type in British Museum.

Hab. NataL. O.R.C. Junction Station (Connolly).

\section{Euonyma standeri, Connolly.}

1910 Euonyma standeri, Conn., A.M.N.H. vi. p. 264. pl. 6, f. 9. D.F. Type in British Museum.

Hab. Transval. Stander's Kop (Connolly).

\section{Euonyma turriforims (Krauss).}

1848 Bulimus turriformis, Krs., Südafr. Moll. p. 78. pl. 5, f. 2. D.F.

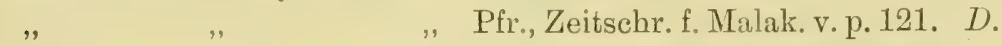
$1853 \quad$ " " " Mon. Hel, iii. p. 392. D.

1898 Opeas turriforme, Krs., Stur., S.A. Moll. p. 60. N.

1906 Euonyma turriformis, Krs., Pilsb., Man. of Conch. xviii. p. 43. pl. 10, f. 77, 78. D.F.

1910 , "Conn., A.M.N.H. vi. p. 256. pl. 6, f. $1 . D . N . F$. 1911

Not Bulimus turiformis, Reeve, 1850. vii.p.224. (Emend.)

Type in Stuttgart Museum.

Hab. Natal (Wahlberg). Widely distributed.

Cape of Good Hope. Port Elizabeth (Crawford). Kowie (Penther). Port St. John's (Shortridge). Grahamstown (var., Farquhar).

Craven's loc., Lydenburg, probably refers to another species.

var. Acus, Morelet.

1889 Stenogyra acus, Morel., J. de C. xxxvii. p. 8. pl. 1, f. 6. D.F. 1896 Subutina glaucocyanea, M. \& P., A.M.N.H. xviii. p. 317. pl. 16, f. 5. D.F.

1906 Euonyma acus, Morel., Pilsb., Man. of Conch. xviii. p. 40. pl. 10, f. 70 . D.F.

, " glaucocyanea, M.\&P.,Pilsb., ibid.,p.43.pl.10,f.76. D.F. $1910 \quad " \quad$ turiformis, Krs., var, acus, Morel. (= glaucocyanea, M. \&P.), Conn., A.M.N.H. vi.p.257.pl.6,f.2. D.N.F. Types in British Museum.

Hab. Cape of Good Hope. Port Elizabeth (Crawford; Fraser). 
var. SARIssa, Pilsbry.

1906 Euonyma turriformis sarissa, Pilsb., Man. of Conch. xviii. p. 44. pl. 10, f. 84,85 . D.F.

1910 , Conn.,A.M.N.H.vi.p.258. N.

Type in Acad. Nat. Sci. Philadelphia.

Hab. Natal (Cassin).

450. Euonyma uniconnis, Connolly.

1910 Enonyma unicornis, Conn., A.M.N.H. vi. p. 265. pl. 6, f. 3. D.F. Type in coll. Connolly.

Hab. Transvant. Schanz Kop, Pretoria (Connolly). Potchefstroom (Miss Livingston).

Cape of Good Hope. Cradock (var., Farquhar).

Orange Eree State. Bloemfontein (var., Connolly).

451. Euonyara varia, Connolly.

1910 Euonyma varia, Conn., A.M.N.H. vi. p. 263.pl. 6, f. 5-7. D.F. Type in British Museum.

Hab. Transvaal. Pienaar's Poort; Pretoria District (Connolly). Potchefstroom (Miss Cachet). Zoutpansberg (var., Cregoe).

Genus ZOOTECUS, Westerlund, 1887.

(Fauna Palæarct. Reg. iii. pp. 3, 75.)

(=Chilogymmus, Jousseaume, 1894.)

Type of Genus, Z. insularis (Ehrnb.).

452. ZоотеCus eulimoides (Gray).

1838 Bulimus eulimoide, Gray, Alexander's Expedition, ii. p. 269. D. Type in British Museum.

Hab. Great Namaland. Near Great Fish River (Alexander).

453. Zootecus namibicus (Böttger).

1910 Ena (Ebumea) namibica, Bttg., Abh. Senckenb. Naturf. Ges.

Frankfurt, xxxii. p. 444. pl. 28, f. 10 . D.F.

Type in Senckenberg Museum, Frankfurt.

Hab. Damaraland, 140 kilom. inland from Swakopmund (Rintelen). 
Sub-FAmily CELIAXIN 王, Pilsbry, 1904.

(Man. of Conch. xvi. p. 194.)

Genus CCELIAXIS, Adams \& Angas, 1865.

(P.Z.S. p. 54.)

(=Bathyaxis, Ancey, 1887, and Sphalerostoma, Girard, 1892.)

Type of Genus, C. layardi, Ad. \& Ang.

454. Celiaxis layardi, Adams \& Angas.

1865 Subulina (Coliaxis) layardi, Ad.\&Ang.,P.Z.S. p.54. pl.2,f.1. D.F. 1868 Bulimus layardi, Ad. \& Ang., Pfr., Mon. Hel. vi. p. 95. D. 1881 Coliaxis " Angas, Layard, P.Z.S. p. 839. N.

1892 Sphalerostoma layardi, Ad. \& Ang., Girard, Jorn. de Sci. Lisbon,

1893

1901 Bathyaxis

1906 Coliaxis ii. p. 245 . N.D.

, ibid. iii. pl. 1, f. 3. F. Ancey, J.de C. xlix. p. 223. $N$. Pilsb., Man. of Conch. xviii. p. 337.pl.49, f.11-14. D.F.

Type-ubi?

Hab. Cape of Good Hope (fide Adams \& Angas). Tharfield (Layard). Kowie (Bowker). Port Alfred; East London (Farquhar). Port Elizabeth (Crawford).

Transvaal. Traces of this, or of a larger, possibly extinct, species have been found at Pienaar's Poort (Connolly).

Tribe ELASMOGNATHA, Mörch, 1863.

(Vidensk. Med. Naturh. Forenh. Copenhagen, p. 267.)

FAMILY SUCCINEID E, Tryon, 1866.

(Amer. Journ. of Conch. ii. p. 222, as Succinidæ, emend. Fischer, 1874, J. de C. xxii. p. 137.)

Genus SUCCINEA, Draparnaud, 1801.

(Tabl. Moll. Fr. p. 32.)

Type of Genus, S. amphibia, Drap. (putris, Lin.).

455. Succinea africana, Krauss.

1848 Succinea amphibia, Drap.,var.africana,Krs.,Südafr.Moll.p.73. N. 1856 " africana, Krs., Rgt., Rev. et Mag. Zool. p. 11. N. 
1856 Succinea africana, Krs., Bgt., Amén. malac. i. p. $136 . \quad$ N. 1909 " " Bgt., Kob., Abh. Senckenb. Naturf. Ges. Frankfurt, xxxii. p. 74 .

Type in Stuttgart Museum.

Hab. Transvade. River Limpopo (Wahlberg).

Note.-The reference to Bourguignat's Amén. malac., quoted above, and for succeeding species of Succinea, is a reprint from the Rev. et Mag. Zool. for the same year. I omit the latter in the following pages.

456. Succinea Arboricola, nom, mut.

1887 Succinea arborea, Mousson, J. de C. xxxv. p. 297. pl. 12, f. 7. D.F. $1904 \quad$. . . . von Mts., Die Kalahari, p. 756. N.

Type in Zurich Museum.

Hab. Bechuanaland. "Kalaruri" (= Kalahari) (Schinz). Lake Ngami; Okavango marshes; Hardekol Drift, Botletle River (Passarge).

As Mousson's name must yield priority to S. arborea, Adams \& Angas, 1863 (P.Z.S. p. 523), from South Australia, a new name is necessary for the present species.

457. Succinea bowkeri, Melv. \& Pons.

1893 Succinea bowkeri, M. \& P., A.M.N.H. xii. p. 110. pl. 3, f. 18. D.F. Type in British Museum.

Hab. Natal. Malvern (Bowker):

Probably identical with S. striata, Krs.; it has not been possible, however, to compare the types.

458. Succinea convoliyi, Preston.

1907 Succinea ? badia, Morel., Dixey \& Longstaff, Trans. Entom. Soc. p. $361 . \quad N$.

1912 " comollyi, Prest., A.M.N.H. ix. p. 445. D.F.

Type in coll. Preston.

Hab. Rhodesia. Rain Forest, Victoria Falls (Dixey \& Longstaff; Connolly, \&c.).

This is in all probability the species referred to in 1907 by Dixey and Longstaff, who remark, "Very near S. putris, Linn."

459. SuCCINEA DAKAËnsis, Sturany.

1898 Succinea dakaënsis, Stur., S.A. Moll. p. 72. pl. 3, f. 52-54. D.F. Type in Naturh. Hofmus. Vienna.

Hab. Rhodesia. Daka River (Penther). 
460. Succinea delalandei, Pfeiffer.

[S.A.M.]

1821 Helix (Cochlohydra) elongata, var. $\gamma$, Fér., Tabl. Syst. Moll. pt. 3. p. 31 (or 27). L., and Hist. Nat. Moll. pl. 11A, f. 11. F.

1851 Succinea delalandii, Pfr., Zeitschr. f. Malak. viii. p. 28. D.

1853 " delalandei ", Mon. Hel. iii. p. 11. D.

1854, 5 " $\quad, \quad$ "Conch. Cab. p. 37. pl. 3, f. 38-40. D.F. 1856 " delalandii ", Bgt., Amén. malac. i. p. 135. D.

Type in British Museum.

Hab. Cape of Good Hope. "Près des marais salés" (Delalande).

Cape Peninsula. “Baszaarms" (Baas Harman's) Kraal (Benson). Hout Bay; Seekoe Vlei ; Maitland (Connolly).

British Bechuanaland. Kuruman (Layard).

Transvaal. Oliphants River (fide Craven).

var. KURRI, von Martens.

1869 Succinea delalandei, Pfr., var. kurri, von Mts., Mal. Blätt. xvi. p. $211 . D$.

Type in Zool. Mus. Berlin.

Hab. "South AFricA" (Kurr).

461. Succinea exarata, Krauss.

1848 Succinea exarata, Krs., Südafr. Moll. p. 74. pl. 4, f. 15. D.F.

\begin{tabular}{|c|c|c|c|}
\hline 185 & " & ,, & Pfr., Mon. Hel. ii. p. 518. D. \\
\hline 185 & , & ", & Bgt., Amén. malac. i. p. 134. $D$. \\
\hline 191 & ", & , & $\begin{array}{l}\text { Bttg., Abb. Senckenb. Naturf. G } \\
\text { Frankfurt, xxxii. p. } 449 . \quad N .\end{array}$ \\
\hline
\end{tabular}

Type in Stuttgart Museum.

Hab. Natal. In swamps (Krauss). Near Pietermaritzburg(Burnup).

Damaraland. Gobabis (subfossil, Hermann, fide Böttger).

462. Succinea moussoni, von Martens.

1887 Succinea, ? Sp., Mouss., J. de C. xxxy. p. 298. N.

1904 " moussoni, von Mts., Die Kalahari, p. 755, f. 2. D.F.

1910 " Sp., Mouss., Bttg., Abh. Senckenb. Naturf. Ges.

Frankfurt, xxxii. p. 449. N.

Type in Zool. Mus. Berlin.

Hab. Ovampoland. Upingtonia, south-east of Ondonga (subfossil, Schinz). Sodanna (Passarge).

Bechuanaland. South of Hardekol Drift, Botletle River, in marly sandstone (Passarge). 
463. Succinea Patentissima, Menke.

1853 Succinea patentissima, Mke., Pfr., Zeitschr.f. Malak. x. p. 52. D.

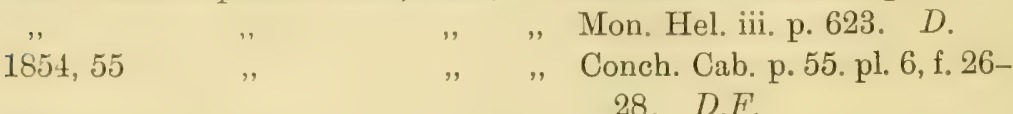

1856 , Bgt., Amén. malac. i. p. 135. D.

Type-ubi?

Hab. Natal. Port Natal (Menke). Umgeni Lagoon (Burnup). Zululand. Lake Sibayi (Toppin).

464. Succinea piscinalis, Melv. \& Pons.

1898 Succinea piscinalis, M. \& P., A.M.N.H. ii. p. 127. pl. 7, f. 4. D.F. Type in British Museum.

Hab. Cape of Good Hope. Fish River (Farquhar).

465. Succinea planti, Pfeiffer.

1856 Succinea planti, Pfr., P.Z.S. p. 326. D.

$1859 \quad$ " $\quad$ " Mon. Hel. iv. p. 805. D.

Type in British Museum.

Hab. Natad. Cape Natal (Plant). Pietermaritzburg (Burnup).

466. Succinea striata, Krauss.

1848 Succinea striata, Krs., Südafr. Moll. p. 73. pl. 4, f. 16. D.F.

\begin{tabular}{|c|c|c|c|}
\hline & ", & ", & Pfr., Zeitschr. f. Malak. v. p. 122. D. \\
\hline 56 & ", & . & n. Hel, 111. p. II. D. \\
\hline & & " & Bgt, Amen. malac. 1. p. 134. $D_{0}$ \\
\hline & & $"$ & Jick Fauna N-O-Afr. pp. 172,173 \\
\hline 10 & 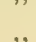 & 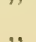 & (var.) Bttg., Abh. Senckenb. Na \\
\hline
\end{tabular}

Type in Stuttgart Museum.

Ges. Frankfurt, xxxii. p. 449. N.

Hab. Transvaal. River Limpopo (Wahlberg). Pretoria (McBean).

Damaraland。 Gobabis (subfossil, Hermann).

Natal. Pietermaritzburg (Burnup).

CApe of Good Hope. Grahamstown (Farquhar). Zuurberg, near Coerney (Crawford).

Orange Free State. Kopjes Siding (Connolly). Bloemfontein (Godfrey).

Also reported from North and Central Africa.

Succinea limicola, Morel. (Ann. Mus. Genova, 1872, iii. p. 191. 
pl. 9, f. 8), described from Abyssinia, is considered by Jickeli to be a variety of the present species. S. bowkeri, M. \& P., and planti, Pfr, are probably identical with striata.

Tribe DITREMATA, Fischer \& Crosse, 1878.

(Mission au Mexique, i. p. 698.)

FAmily VERONICELLID Æ, Gray, 1840.

(Syn. Brit. Mus. pp. 126, 149.)

Genus VeroniceLLA, de Blainville, 1817.

(Journ. de Physique, Ixxxv。 p. 440.)

Type of Genus, $V$. lavis, de Blainville.

467. Veronicella maura (Heynemann).

1885 Vaginula maura, Heynem., Jahrb. d. Deutsch. Mal. Ges. xii. pp. 7, 104. pl. 1, f. 6, 7. D.F.

Type in British Museum.

Hab. Lorenzo Marques. Delagoa Bay (Mrs. Monteiro).

468. Veronicelda natalensis (von Rapp).

1848 Vaginulus natalensis, von Rapp, Krs., Südafr. Moll. p. 72. D.

1855 Limax natalensis, "Krs.," H. \& A. Ad., Gen. rec. Moll. ii. p. 219. $1862 \quad$ " $\quad$ " Heynem., Mal. Blätt. ix. p. 217. N. 1879 Vaginula natalensis, von Rapp, Gibb.,Journ.of Conch.ii. p.140. N. 1885 " " Heynem., Jahrb. d. Deutsch. Mal. Ges. xii. p. 103. D.

" Limax natalensis, Krs. (is Vaginula ditto), Tryon, Man. of Conch. i. p. 214.

1893 Veronicella natalensis, von Rapp, Ckll., Conchologist,ii. p.216. N. $1910 \quad$ " $\quad$ Cllge.,Ann.NatalMus.ii.p.170. N.

Type in Stuttgart Museum.

Hab. Natal (Krauss). Port Shepstone (Burnup).

Cape of Good Hope. Kowie District (Penther).

469. Veronicella petersi (von Martens).

1879 Vaginula petersi, von Mts., Monats-Ber. K. Preuss. Akad. Wiss. Berlin, p. $736 . \quad D$. 
Type in Zool. Mus. Berlin.

Hab. Lorenzo Marques. Inhambane (Peters).

470. Veronicelda saxicola, Cockerell.

1893 Veronicella saxicola, Ckll., Conchologist, ii. pp. 194, $216 . \quad D$.

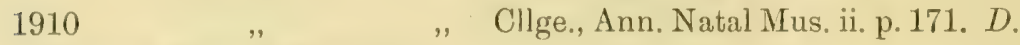

Type in British Museum.

Hub. Cape of Good Hope. Port Elizabeth (Craven).

Natal. Pietermaritzburg (Burnup).

FAMILY ONCHIDIID 2 , Gray, 1824.

(Ann. of Philos. viii. p. 108, as Onchidiadæ, emend. H. \& A. Adams, 1855, Gen. rec. Moll, ii. p. 232.)

Genus ONCHIDIUM, Buchanan, 1800.

(Linn. Trans. v. p. 132.)

Type of Genus, O. typha, Buch.

471. Onchidium Burnupi, Collinge.

1902 Onchidinm burnupi, Cllge., Journ. of Malac. ix. p. 17, f. 1, 2. D.F. $1910 \quad$, $\quad$ "Ann. Natal Mus. ii. p. 171. D.

Type in University Museum of Zoology, Cambridge.

Hab. Natal. Umlaas Lagoon (Burnup).

472. Onchidium Peroni, Cuvier.

1804 Onchidium peronii, Cuv., Ann. Mus. Nat. Hist. Paris, v. p. 38. pl. 6, f. 1-9. D.A.

1817

Mém. à l'Hist. et à l'Anat. Moll. 13. p. 1. pl. 1, f. 1-9. D.A.

1821

1822

Fér., Tabl. Syst. Moll. pt. 2. p. 6. N.

1825 Peronia mauritziand, de Blainv., Man. de Malac. p. 489. pl. 46 (1827), f. 7. F.

1832 Onchidium tonganum, Quoy \& Gaim., Voy. Astrolabe, Moll. p. 210. pl. 15, f. 17, 18. D.F.

$1836 \quad$ " peronii,Cuv.,Desh.,Hist.nat.An.s. Vert.vii.p.709. N. " $\quad$ " tonganum, Quoy \& Gaim., Desh., ibid.p. 709. D.N. $1848 \quad$ " peronii, Cuv., Krs., Südafr. Moll. p. 72. N.

1870 " tonganum, Quoy \& Gaim.(? O. peronii, Cuv.), Semp., Reis. im Arch. Philippin. ii, 3. p. 258. pl. 19, f. 2, 9. pl. 22, f. 1, 2, 10. D.F.A. 
1893 Oncidium peroni, Cuv. (=O. melanopneumon, Bergh, and tonganum, Q. \& G.), Plate, Zool. Jahrb. Jena, vii, 1. p. 172. pl. 12, f. 85 . D.F.

1910 ", tonganum, Cunningham, Encycl. Brit. 11th Ed. xi.

Type-ubi? p. 525 , f. 62 . F.

Hab. Natal coast (Krauss). Congella, near Durban (Burnup).

Lorenzo Marques. Inhambane (Peters).

Cape Peninsula. Green Point (Purcell).

Described from Mauritius.

473. Onchidium savignyi, Semper.

1870 Onchidium savignyi, Semp., Reis. im Arch. Philippin. ii, 3. p. 260. pl. 19, f. 6. pl. 20, f. 1. pl. 22, f. 5-9. D.F.A.

1903 Onchidium savignyi, Semp., Smith, Proc. Mal. Soc. v. p. 401. N. $1910 \quad$ " $\quad$ Cllge., Ann. Natal Mus. ii. p. 172. L.

Type-ubi?

Hab. NataL. Scottburgh (Burnup).

Genus ONCHIDELLA, Gray, 1850.

(Fig. Moll. Anim. iv. p. 117.)

Type of Genus, O. nigricans (Q.\& G.).

474. Onchidella maculata, Plate.

1893 Oncidiella maculata, Plate, Zool. Jahrb. Jena, vii, 1. p. 201.pl. 7, f. 4. pl. 9 , f. 43,44 . pl. 10 , f. $45-49$, 52. pl. 11, f. 68 . pl. 12 , f. 101 . D.F.A.

Type-ubi?

Hab. Great Namaland. Angra Pequena (fide Plate).

Sub-Order BASOMMATOPHORA, Keferstein, 1865.

(Bronn's Thier-reichs, iii. p. 1246.)

T'Ribe GEHYDROPHILA, Férussac, 1821.

(Tabl. Syst. Moll. pt. 3. p. 95 (or 91), as Géhydrophiles.)

FAmily AURICULIDA, Gray, 1824.

(Ann. of Philos. viii. p. 107, as Auriculadæ, emend. 1840, Turton's Manual, p. 101.) (=Ellobiidæ, H. \& A. Adams, 1855.)

SUB-FAMILY MELAMPINE, H. \& A. Adams, 1855,

(Gen. rec. Moll. ii. p. 242,) 
Genus MELAMPUS, de Montfort, 1810.

(Conch. Syst. ii. p. 319.)

(=Conovulus, Lamarck, 1812, \&c.)

Type of Genus, M. coniformis (Brug.).

Recent classification of this Genus is somewhat unsatisfactory: species from widely distant localities can, certainly, be placed together in well-marked groups, members of which have of late year's been placed in synonymy; but it appears almost impossible that these inoperculate, brackish-water pulmonates can travel such immense distances as, say, from the Sandwich Islands to Natal, and it is in the highest degree unlikely that the union of shells from such localities under the same name can be correct.

On these grounds I have preferred to retain, as far as possible, names of species described by the older writers from South Africa for specimens recently collected there, although faulty figures and lack of authentic examples render their correct identification by no neans certain.

475. Melampus acinoides, Morelet.

1889 Melampus acinoides, Morel., J. de C. xxxvii, p. 14. pl. 1, f. 9. D.F. 1898 Kob., Conch. Cab. p. 205. pl. 23, f. $12,13 . \quad D . F$.

Type in British Museum.

Hab. Cape of Good Hope. Zwartkops River, near Port Elizabeth (Crawford). Kalk Bay (Lightfoot).

476. Melampus Caffer (Küster).

1844 Auricula caffra, Küst., Conch. Cab. p. 36. pl. 5 (1843), f. 7. D.F. 1854 Melampus ater, Mühlf. (=caffer, Küst.), H. \& A. Ad., P.Z.S. p.10. 1856 ", caffer, Küst. (=ater, Mühlf.), Pfr., Mon. Auric.p.40. D.

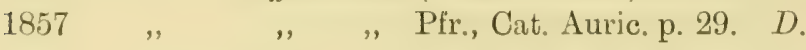

$1871 \quad$ " , " von Mts. \& Langkavel, Südsee-Conch. p. 56. pl. 3, f. $11 . \quad F$.

1878 Auricula caffra "Sow., Conch. Icon. pl. 7, f. 53. D.F.

1884 Melampus caffer , Garrett, Journ. Acad. Nat. Sci. Phila. ix. p. 89. D.N.

$1890 \quad$," Krs., Mlldff., Ber. Senckenb. Naturf. Ges. Frankfurt, p. 254. N.

Type in Stuttgart Museum.

Hab. NataL. Mouth of Umlaas River (Krauss). 
It is doubtful whether many of the foregoing references, which record $M$. caffer from all parts of the Indian and Pacific Oceans, really relate to Küster's species.

\section{var. MINOR, Küster.}

1839 Conovulus ater, Mühlf., Anton, Verz. Conch. Samml. p. 48 (without characters).

1844 Auricula caffra, var. minor, Küst., Conch. Cab. p. 36. pl. 5 (1843), f. 6,8 . D.F.

Described from Ohetaroa, and probably quite distinct from the South African form.

\section{Melampus küsteri (Krauss).}

1842 ?? Auricula monile, Lam., Rve., Conch. Syst.ii.p.106.pl.187,f.8. F. 1844 ", kiisteri, Krs., Küst., Conch. Cab. p. 34. pl. 4 (1843),

$$
\text { f. 10-13. D.F. }
$$

1856 Melampus $\quad$, , $\quad$ Pfr., Mon. Auric. p. $33 . \quad$ D.

$1857 \quad$ " $\quad$, " , , Cat. Auric. p. 23. D.

Type in Stuttgart Museum.

Hab. Natal. Mouth of Umlaas River (Krauss).

var. oelongus, Küster.

1844 Auricula kïsteri, Krs., var. oblonga, Küst., Conch. Cab. p. 34. D. Hab. NataL. Mouth of Umlaas River (Krauss).

478. Melampus uividus (Deshayes).

1830 Auricula livida, Desh., Enc. Méth. Vers, ii. p. 91. D.

1838 ," " Hist. nat. An. s. Vert. viii. p. 338. D.

$1844 \quad$," Küst., Conch. Cab. p. 44. pl. 6 (1843), f. 21. I). $F$.

$1848 \quad$," , Krs., Südafr. Moll. p. 81. N.

1854 Melampus lividus, Lin. (=livida, Desh.), H.\&A.Ad., P.Z.S. p. 10.

1856 , N Desh., Pfr., Non. Auric. p. 40. D.

$18.57 \quad$," „, , Cat. Auric. p. 29. D.

1878 Auricula livida, Lin., Sow., Conch. Tcon. pl. 7, f. 58. D.F.

1882 Mclampus lividus, Desh., Morel., J. de C. xxx. p. 101. N.

$1897 \quad$ ", $\quad$ von Mts., D.-O.-Afr. p. 264. D.

Specimens ex auct. in École des Mines, Paris.

Hab. Natal. Mouth of Umlaas River (Krauss; Burnup). ? juv. in Durban Bay (Burnup).

Also chronicled from Mayotte, Réunion, Mauritius, Seychelles, \&c. 
It is impossible to regard this species as in any way connected with Bulla livida, Lin. (Syst. Nat., Ed. 10. i. p. 729) \& Gault. (Index Test. pl. 25, f. B), which appears to represent a different Genus.

varr. cœruleus, fasciatus, and ovatus, Küster.

1844 Auricula livida, Desh., varr. corulea, ovata, and fasciata, Küst., Conch. Cab. p. 45 . pl. 6 (1843), f. 22-26. D.F.

Hab. Natal coast (Krauss).

479. Melampus ordinarius, Melv. \& Pons.

1901 Melanpus ordinarine, M.\&P., A.M.N.H.viii.p.321.pl.2,f.14. D.F. Type in British Museum.

Hab. NataL. Mouth of Umlaas River (Burnup).

480. Melaitpus parvulus, Nuttall.

1854 Melampus parvulus, Nutt., Pfr., Mal. Blätt. i.p. 147 (without characters).

1856

1857

1871

" Mon. Auric. p. 24. D.

1898

$\begin{array}{lll}, \quad & \quad, \quad \text { Mon. Auric. p. } 24 . \quad D \\ , \quad & \text { " } \quad \text {, } & \text { Cat. Auric. p. } 16 . \quad D .\end{array}$

von Mts. \& Langkavel, SüdseeConch. p. 56. pl. 3, f. $10 . \quad F$. Kob., Conch. Cab. p. 220. pl. 26, f. 5. D.F.

Originals in British Museum.

Hab. NataL. Durban Bay; mouths of Umlaas and Umkomaas Rivers (Burnup).

Owing to the diverse geographical distribution, it is most unlikely that these Natal specimens are conspecific with the true parvulus, which was described from Oahu; but, in the series examined, I have been unable to find valid conchological grounds for their separation. Is it possible that the species can have been introduced alive in ballast?

481. Melampus semiaratus, sp. nov., pl. 2, f. 8 . [S.A.M.] 1898 Melampus granifer, "Mouss.," M. \& P., Proc. Mal. Soc.iii.p.180. L.

Shell small, conic-ovate, subrimate, solid, rather glossy, of uniform dark brown colour, the upper and lower portions covered with spiral grooves which are crossed by faint transverse strix; the middle portion is destitute of sulcation, and thus has a comparatively smooth appearance. Spixe short, conical; apsx acute, 6 tlat 
whorls, the last comprising practically the entire shell. Aperture long and narrow, furnished with one receding white rib half-way up the outer lip; a fold of medium size at the base of the columella; and three sharp white parietal plaits at almost equal, but gradually decreasing distances between the columellar fold and the top of the aperture. Peristome acute, of paler colour. Columellar margin thickly reflexed, almost adnate.

Shell $9.6 \times 5.5$; aperture 7.5 ; last whorl $8.8 \mathrm{~mm}$.

Type in British Museum.

Hab. NataL. Durban Bay; mouths of Umlaas and Umkomaas Rivers (Burnup).

A member of the group comprising $M I$. granifer, Mouss., from Java; sulculosus, von Mts., from Amboina; corticinus, Morelet, from Mauritius ; striatus, Pease, from Tahiti, and scmisulcatus, Mouss., from Samoa, to the last two of which it must be very nearly allied. The half-furrowed appearance of the new species, though usual, is not always constant, some shells being covered all over with spiral grooving, in which state they much resemble striatus; but owing to the widely divergent distribution, it is most improbable that the three species can be the same.

\section{Melaipus umlaasianus (Krauss).}

1814 Auricula umlaasiana, Krs., Küst., Conch. Cab. p. 43. pl. 6 (1843), f. 16-18. D.F.

1856 Melampus umlaasianus, Krs., Pfr., Mon. Auric. p. 34. D. 1857

$$
\text { , " Cat. Auric. p. 24. D. }
$$

Not Auricula umlassiana, "Krs.," Sow., Conch. Icon, pl. 6, f. 48.

Type in Stuttgart Museum.

Hab. Natal. Mouth of Umlaas River (Krauss).

var. oBscurus, Küster.

1844 Auricula umlaasiana, Krs., var. obscura, Küst., Conch. Cab.

$$
\text { p. } 44 \text {. pl. } 6 \text { (1843), f. 19, 20. D.F. }
$$

Hab. Natal. Mouth of Umlaas River (Krauss). Isipingo (Burnup).

Specimens from Isipingo, apparently referable to this variety, have of late year's been erroneously attributed to $M$. castaneus, Mühlf. ( $=A$. fusca, Phil.), a Sandwich Island species. M. avellana, Morelet, from Mauritius, is also near akin, but it appears inadvisable to place either of the three in synonymy. 
Genus MARINULA, King, 1835.

(Zool. Journ. v. p. 343.)

Type of Genus, $M$. pepita, King.

483. Marinula, ? Sp.

Hab. Cape of Good Hope. Camps Bay (McBean). Dassen Island (Lightfoot).

Two specimens only of Marimula have so far been collected in South Africa. They closely resemble a form from Tristan da Cunba, which has been attributed in the British Museum and elsewhere to M. pepita, King (=nigra, Phil., \& marinella, "King," Küster). More material is required before they can be determined.

Genus PEDIPES, Férussac, 1821.

(Adanson, Hist. nat. du Sénégal, 1757, pt. 2, p. 11 (pre-Linnean), and Férussac, Tabl. Syst. Moll., 1821, pt. 3. p. 103 (or 99).)

Type of Genus, P. afer (Gmel.).

484. Pedipes affinis, Férussac.

1821 Pedipes affinis, Fér., Tabl. Syst. Moll. pt. 3. p. 113 (or 109). D. 1856

1857

" Pfr., Mon. Auric. p. 72 . D.

1863

" $\quad$ Cat. Auric. p. 54. N.

" Desh., Notes sur l'Ile Réunion, ii, E. p. 83. pl. 37, f. 5,6 . D.F.

1874 Lamodonta affinis,Fér.,Jick.,FaunaN.-O.-Afr.p.181.pl.7,f.6. D.F. 1887 Pedipes affinis, Fér., Ancey, Bull. Soc. Mal. France, iv. p. 285. N. 1898 Melampus (Laimodonta) affinis, Fér., Kob., Conch. Cab. p. 202. pl. 23, f. 7,8 . D.F.

1900 Pedipes affinis, Fér., Kob., Conch. Cab. p. 259. D.

Type-ubi?

Hab. Natal. Durban (Burnup).

Described from Mauritius, and known from the Red Sea.

Sub-FAmily AURICULIN E, Pfeiffer, 1857. (Cat. Auric. p. 54.)

Genus AURICULASTRA, von Martens, 1880.

(Meeresfauna Mauritius, p. 207.)

Type of Genus, A. clongata (Parr.). 
485. Auriculastra catonis (Melv. \& Pons.).

1899 Auricula catonis, M. \& P., A.M.N.H. iv. p. 199. pl. 3, f. 13. D.F. 1900

Type in British Museum.

Kob., Conch. Cab. p. 265. pl. 31, f. 14. D.F.

Hab. Natal. Cato's Creek, Durban; mouths of Umlaas and Umkomaas Rivers (Burnup).

486. Auriculastra radiolata (Morelet).

[S.A.M.]

1848 Auricula pellucens, "Mke.," Krs., Südafr. Moll. p. 82. N.

1860 Melampus radiolatus, Morel., Séries Conch. ii.p.93.pl.6,f.11. D.F

1876 Auriculus radiolatus, Morel., Pfr., Mon. Pneum. iv. p. 359. D. 1877 Auricula radiolata, Morel., Nev., Hand List, i. p. $226 . \quad L$.

1897 Auriculastra radiolata, Morel., von Mts., D.-O.-Afr. p. 264. N 1899 Auriculadurbanica,M.\&P.,A.M.N.H.iv.pp.193,199.pl.3,f.14.D.F 1900

Kob., Conch. Cab. p. 264. pl. 31, f. 13. D.F.

Types of radiolata and durbanica in British Museum.

Hab. Natal (? pellucens, Wahlberg; radiolata, Nevill). Cato's Creek, Durban; mouths of Umlaas and Umkomaas Rivers (durbanica, Burnup).

Radiolata was described from Zanzibar, durbanica from Natal; the type of the former has not attained fullest dimensions, but seems inseparable from similar specimens of durbanica.

Genus CASSidula, Férussac, 1821.

(Tabl. Syst. Moll. pt. 3. p. 109 (or 105).)

(=Rhodostoma, Swainson, and Sidula, Gray, 1840.)

Type of Genus, C. auris felis (Brug.).

487. Cassidula labrella (Deshayes).

1830 Auricula labrella, Desh., Enc. Méth. Vers, ii. p. $92 . \quad$ D.

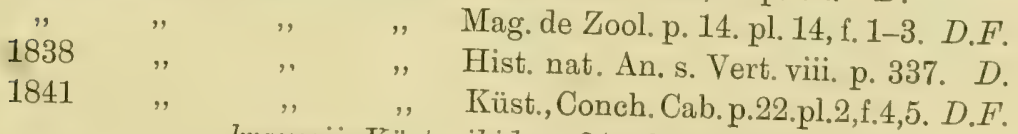

$1848 \quad$ " kraussii, Küst., ibid. p. 24. pl. 3, f. 6-8. D.F.

" " " Krs., Südafr. Moll. p. 82。 N

1856 Cassidula kraussi, Küst., Pfr., Mon. Auric. p. 113. D.

" $\quad$ " lutescens, Pfr., Mon. Auric. p. 113. D.

1857 " - labrella, Desh., Pfr., Mon. Auric. p. 112. D. 
1857 Cassidula kranssi, Küst., Pfr., Cat. Auric. p. 86. D. 1874 ", labrella, Desh. (=kraussi and lutescens), Jick., Fauna N.-O.-Afr. p. $186 . D$.

1878 Auricula kraussii, Küst., Sow., Conch. Icon. pl. 5, f. 32 . D.F. 1883 Cassillula labrella, Desh., Bgt., Ann. Sci. nat. Paris, xv.p. 124. N.

Type of kraussi in Stuttgart Museum ; labrella-ubi?

Hab. NataL. Mouth of Umlaas River (kraussi, Krauss). Durban (Burnup).

Cape of Good Hope. Zwartkops River marshes, near Port Elizabeth (Crawford).

Labrella was described from Mauritius, lcraussi from Natal, and the Hab. of lutescens was unknown.

Genus PHYTIA, Gray, 1821.

(London Medical Repository, xv. p. 231.)

(=Alexia, Leach in Gray, 1847, nec Stephens, in Coleoptera 1835.)

Type of Genus, P. denticulata, Mont. (myosotis, Drap.).

488. Phytia acuminata, Morelet.

[S.A.M.]

1889 Alexia acuminata, Morel., J. de C. xxxvii. p. 15. pl. 1, f. 11. D.F. " " pulchella " $\quad$ " $\quad$ p. 15. pl. 1, f. 10. D.F. $1898 \quad$ " , $\quad$ ". Kob., Conch. Cab. p. 133. pl. 19, f. 7, 8. D.F.

" " acuminata ", Kob., ibid.p.133.pl. 19, f. 13, 14. D.F.

Types in British Museum.

Hab. Cape of Good Hope. Port Elizabeth (Crawford; Farquhar).

The above species were founded on very immature shells, and, in a large series, merge into one another. The type of acuminata measures $4.5 \times 2 \mathrm{~mm}$., while the largest examples that I have examined from Port Elizabeth are $20 \times 4$ and $19.5 \times 5 \mathrm{~mm}$. respectively; there seems no doubt, however, that these are the adult of Morelet's species. They are closely allied to the variable European Phytia myosotis (Draparnaud).

Tribe HYGROPHILA, Férussac, 1822.

(Tabl. Syst. Moll., pt. 1. p. xxxiii, as Hygrophiles.)

FAMILY LIMN EID正, Gray, 1824.

(Ann. of Philos. viii. p. 108, as Lymneadæ, emend. Broderip, 1839, Penny Cyclopædia, xiii. p. 497.) 
GENus LIMN EAA, Lamarck, 1799.

(Ném. Soc. Hist. Nat. Paris, p. 75, as Lymnæa, emend. Rang, 1829, Man. de l'Hist. nat., Moll. p. 176.)

Type of Genus, L. stagnalis (Lin.).

489. Limnat Dakä̈nsis, Stuiany.

1898 Limnœus dakaënsis, Stur., S.A. Moll. p. 74. pl. 3, f. 55, 56. D.F. Type in Naturh. Hofmus. Vienna.

Hab. Rhodesia. Daka (Penther).

490. Liminata damarana, Böttger.

1910 Limnaa damarana, Bttg., Abh. Senckenb. Naturf. Ges. Frankfurt, xxxii. p. 4 ย0. pl. 28, f. 16. D.F.

Type in Senckenberg Mus. Frankfurt.

Hab. Damaraland. Gobabis (subfossil, Hermann).

Founded on two specimens only.

491. Limnata natalensis, Krauss.

[S.A.M.]

1848 Limnans natalensis, Krs., Südafr. Moll. p. 85. pl. 5, f. 15. D.F. 1862

" Küst., Conch. Cab. p. 31. pl. 6, f. 1-3. D.F.

1870 Limncea

, Blanford, Obs. Geol. \& Zool. Abyss. p. 472. $N$.

1872

1874

1881

1904

,

,

,

,

,

Type in Stuttgart Museum.

Sow., Conch. Icon. pl. 7, f. 46. D.F.

" Jick., Fauna N.-O.-Afr. p. 190. D.

" Smith, P.Z.S. p. 295. N.

" „, Proc. Mal. Soc. vi. p. 98. N.

Hab. Natal. "Common" (Krauss). An unusually large form is known from the Botanical Gardens, Durban.

Transvaal. Pretoria District (McBean). Zoutpansberg (Cregoe).

Lorenzo Marques. Itschonogove (fide von Martens). Rikatla (Junod).

Orange Free State. Valsch River, Lindley; Rhenoster River, near Heilbron (Connolly). Kroonstad (Eckersley).

CAPE OF Good Hope. Port Elizabeth (Crawford).

Griqualand West. Modder and Vaal Rivers, near Kimberley (Miss Wilman).

Bechuanaland. Lake Ngami (Woosman).

Rhodesia. Zambesi River, above Victoria Falls (Connolly).

Recorded from most parts of Africa. 
Smith (1904) remarks that L. africana, alexandrina, debaizei, jouberti, laurenti, and lavigeriana, Bgt., are probably all forms of natalensis, but Pallary (Mém. Inst. Égypt., 1909, vi, 1. p. 47) considers alexandrina (=natalensis, Auctt., nec Krs.) to be distinct.

var. Exsenta, von Martens.

1866 Limneus natalensis, Krrs., var. exsertus, von Mits., Mal. Blätt. xiii. p. 101. pl. 3, f. 8, 9. D.F.

1874 Limnce natalensis, Krs., var. exserta, von Mts., Jick., Fauna N.-O.-Afr. p. $191 . D$.

$1883 \quad$ " cxserta, Bgt., Ann. Sei. nat. Paris, xv. pp. 40, 125. N.

Described from Abyssinia.

var. orophila, Morelet.

1868 Limnea orophila, Morel., Voy. Welwitsch, Moll.p.87.pl.7,f.4. D.F. 1874 " natalcnsis, Krs., var. orophila, Morel., Jick., Fauna N.-O.-Ifr. p. 191, pl. 3, f. 1. I).li.

Deseribed from Benguela, and also recorded from Abyssinia.

492. Limnea subtruncatula, Böttger.

1910 Limnea subtruncatula, Bttg., Abh. Senckenb. Naturf. Ges. Frankfurt, xxxii. p. 451. pl. ‘8, f. 17. 1).F'.

Type in Senckenberg Mus. Frankfurt.

Hab. Damaraland. Gobabis (subfossil, Hermann).

Founded on three specimens.

493. Limnera truncatula (Müll.).

[S.A.MI.

1774 Buccinum truncatulum, Müll., Verm. ii.p. 130. D.

1789 Bulimus truncatus, Brug., Enc. Méth. Vers, i. p. 310. D.

1801 Limneus minuta, Drap., Tabl. Moll. Fr. p. 51. D.

1803 Helix fossaria, Mont, Test. Brit. ii. p. 372. pl. 16, f. 9. D.F.

1821 Limneus minutus, Drap., C. Pfr., Syst. Anordn. d. deutsch. L. u. W.-Schneck. p. 93. pl. 4, f. 27. D.F.

1829 Iymncea minuta, Drap., Schubert \& Wagner, Conch. Cab. xii. p. 182. pl. 235 , f. 4134,5 . D.F.

1840 Limnces truncatulus, Müll., Gray, Turton's Manual, p. 240. D. and Synomymy.

1855 Limnce truncatula, Müll., Moq.-Tand., Hist. Nat. Moll. Fr. ii. p. 473. pl. 34, f. 21-24. D.17.

1862 Limnaus truncatulus, Müll., Küst., Conch. Cab. p. 17. pl. 3, f. 24-27. D.F. 
1862 Limnœus umlaasianus, Küst., ibid.p. 32. pl. 6, f. 4, 5. D.F. 1872 Limnaa truncatula, Müll., Sow., Conch. Icon. pl. 1, f. 3. D.F. 1883 " " " (=umlaasianus, Küst.), Bgt., Ann. Sci.nat. Paris, xv.pp.86,97,125. N. 1897 " ? truncatula ,, von Mts., D.-O.-Afr. p. 137. N. 1898 Limncens umlaasianus, Küst., Stur., S.A. Moll. p. 74. N. 1899 Limnaa umlaasiana, Küst., M. \& P., A.M.N.H. iv. p. 193. N. Type-ubi?

Hab. Natal. Umlaas River (umlaasiana, in coll. Küster).

Transvaal. Pretoria (McBean).

Cape of Good Hope. Stellenbosch (Péringuey).

Genus PLANORBIS, Geoffroy, 1767.

(Coquilles de Paris, pp. 12, 81.) (=Planorbis, Guettard, 1756, pre-Limnean.)

Type of Genus, $P l$. corneus (Lin.).

494. Planorbis anderssoni, Ancey.

1890 Planorbis anderssoni, Ancey, Bull. Soc. Mal. Fr. vii. p. 161. D. 1898 Stur., S.A. Moll. p. 77. N.D.

Type in coll. Putzeys.

Hab. Ovampoland. Ovambonde (Andersson; Chapman).

Natal. Durban (Penther).

CAPE of Good Hope. Burnt Kraal, near Grahamstown (Farquhar).

$$
\text { 495. Planorbis costulatus, Krauss. }
$$

1848 Planorbis costulatus, Krs., Südafr. Moll. p. 83. pl. 5, f. 8. D.F. 1874 " (var.), Jick., Fauna N.-O.-Afr. p. 219. pl. 7, f. 22, $23 . \quad$ D.N.F.

1877

1884

$$
\text { " }
$$

,

, "Sow., Conch. Icon. pl. 4, f. 26. D.F.

Type in Stuttgart Museum. ", Cless., Conch. Cab. p. 131. pl. 21 (1883), f. 2. D.F.

Hab. Natal. Umgeni Valley (Krauss). Equeefa (Burnup). Zululand. Enseleni River (var., Burnup).

Also reported from Abyssinia.

496. Planorbis crawfordi, Melv. \& Pons.

1893 Planorbis crawfordi, M.\&P., A.M.N.H.xii.p.111.pl.3, f. 20. D.F. Type in British Museum.

Hab. Cape of Good Hope. Van Staaden's River; Zwartkops River, Port Elizabeth (Crawford). 
Transvaal. Ranjesfontein; Potchefstroom (Connolly).

Also recorded from Victoria Nyanza.

497. Planorbis gibionsi, Nelson.

1878 Planorbis gibbonsi, Nels., Quart. Journ. of Conch. i. p. 379. pl. 4, f. 3. D.F. " von Mts., D.-O.-Afr. p. 150. N.

1908

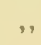
, Neuville \& Anthony, Ann. Sci. nat.

Type in coll. Nelson. Paris, viii. p. 260. N.

Hab. Cape Peninsula. Black River, Maitland (Connolly).

Described from Zanzibar, and known from Central Africa.

None of the original specimens of $P l$, gibbonsi have been available for comparison, while the original description and figure do not appear to quite fit the single representative of this species (ex coll. von Martens) in the British Museum, or similar shells in my collection from South and Central Africa. I have, however, submitted a set of the latter to Mr. J. W. Taylor, who kindly replied: "I have carefully examined the Planorbes you sent, and am of opinion that they do represent the $P l$. gibbonsi of Nelson. I find the lithographic artist made the shape of the mouth in the original figure more angular than I am sure it was. I was much interested in these shells when Gibbons sent them, and gave them considerable attention."

\section{Planorbis hermanni, Böttger.}

1910 Planorbis (Coretus) hermanni, Bttg., Abh. Senckenb. Naturf. Ges. Frankfurt, xxxii. p. 452. pl. 28, f. 18. D.F.

Type in Senckenberg Mus. Frankfurt.

Hab. Damaratand. Okaputa Pan (several dead shells, Hermann). "Very like P. pfeifferi, Krs." (Bttg.).

499. Planorbis leucochilus, Melv. \& Pons.

1903 Planorbis leucochilus, M.\&P., A.M.N.H.xii.p.607.pl.31, f.3. D.F. Type in British Museum.

Hab. NataL. Killarney Lake, Pietermaritzburg (Burnup).

500. Planorbis natalensis, Krauss.

1848 Planorbis natalensis, Krs., Südafr. Moll. p. 83. pl. 5, f. 9. D.F. 1877 " natalis, Krs., Sow., Conch. Icon. pl. 4, f. $32 . \quad$ D.F. 1883 " natalensis, Krs., Cless., Conch. Cab. p. 109. pl. 17 (1882), f. 3. D.F. 
1910 Planorbis (Gyranlus) natalensis, Krs., Bttg., Abb. Senckenb. Naturf. Ges. Frankfurt, xxxii. p. 451. N.

Type in Stuttgart Museum.

Hab. Natal. Umgeni Valley (Krauss).

Cape of Good Hope. Port Elizabeth (fide Morelet).

Damaraland. Gobabis (subfossil, Hermann).

British Bechuanaland. Witkop (subfossil, Schultze).

501. Planorbis pfeifferi, Krauss.

[S.A.M.]

1848 Planorbis pfeifferi, Krs., Südafr. Moll. p. 83. pl. 5, f. 7. D.F. 1877

1882 ", Sow., Conch. Icon. pl. 4, f. 33. D.F. "Cless., Conch. Cab. p. 87. pl. 10 (1850), f. 26-28. D.F.

1893 Planorbis bowkeri, M. \& P., A.M.N.H. xii. p. 111. pl. 3, f. 19. D.F. 1910 " "c.f. pfeifferi, Krs.," Bttg., Abh. Senckenb. Naturf. Ges. Frankfurt, xxxii. p. 451. N.

Co-type of pfeifferi in Stockholm Museum; type of bowkeri in British Museum.

Hab. Natal. Umgeni Valley (Krauss). Clairmont; Umbogintwini River (Burnup).

Zululand (Burnup).

Lorenzo Marques. Itschongove (fide von Martens).

Rhodesia. Daka River (Penther). Salisbury (Miss Weineck).

Transvaal. "Northern Transvaal" (bowkeri, Bowker). Ranjesfontein and Crocodile River, Pretoria District (Connolly).

British Bechuanaland. Witkop (subfossil, Schultze).

Melvill and Standen (Manchester Memoirs, li, 4. 1907, p. 7) apparently refer to some other species under the name of bowkeri; the type appears inseparable from pfeifferi, Krs.

I am not sufficiently weil acquainted with $\mathrm{Pl}$. riïppelli, Dkr., or salinarum, Morel., to confirm the synonymy suggested elsewhere by von Martens and Jickeli.

502. Planorbis rüPpelli, Dunker.

1848 Planorbis rïppellii, Dkr., P.Z.S. p. $42 . \quad D$.

1850 " rïppelli " Conch.Cab.p.41.pl.5,f.10-12. D.F. 1866 " riippellii " von Mts., Mal. Blätt. xiii. p. 4. N.

$1869 \quad$ " $\quad$ " $\quad$ (= salinarum, Morel.), von Mts., Mal. Blätt. xvi.p. 211. D.

$1870 \quad$ " , " Blanf., Obs. Geol. and Zool. Abyss. p. $473 . N$. 
1872 Planorbis rüppelli, Dkr., Morel., Ann. Mus. Genova, iii. p. 207. N. 1874 " " " Jick,, Fauna N.-O.-Afr. p. 211. pl.7, f. $17,18 . D . F$.

$1883 \quad$ " $\quad$ " , Bgt., Ann. Sci. nat. Paris, xv. pp. 100, 127. D.

1900 „, „N Krs., Junod, Bull. Soc. Vaudoise, xxxv. p. $279 . L$.

1908 " " Dkr., Neuville \& Anthony, Ann. Sci. nat. Paris, viii. pp. 249-253. N.F.

Type in Zool. Mus. Berlin.

Hab. Lorenzo Marques. Rikatla (Junod).

Described from Abyssinia.

Jickeli (1874) remarks: "I could not unite Plan. salinarum, Morel., from West Africa, with riippellii; according to the figure in Voy. Wellwitsch, the former differs through less height combined with greater diameter. On the other hand, our Abyssinian Planorbis appears to me very nearly allied to Plan. pfeifferi, Krs. ; comparison with examples of this species, which I do not possess, ought to produce synonymy of the two."

503. Planorbis salinarum, Morelet.

1868 Planorbis salinarum, Morel., Voy. Welwitsch, Moll.p. 85. pl. 5, f. 4. D.F.

$1904 \quad$, , $\quad$ von Mts., Die Kalahari, p. 756. N.

Type in British Museum.

Hab. Bechuanaland. Lake Ngami; Sodanna (subfossil, Passarge).

Described from Dungo saltings, Angola.

Genus SEGMENTINA, Fleming, 1818.

(Encyc. Brit., Suppl. to 4 th, 5th, and 6 th editions, iii. p. 309.)

Type of Genus, Nautilus lacustris, Lightfoot (nitida, Müll.).

504. Segmentina emicans, Melv. \& Pons.

1892 Planorbis (Segmentina) emicans, M. \& P., A.M.N.H. x. p. 241. pl. 3, f. 13. D.F.

Type in British Museum.

Hab. CAPE of Good Hope. Port Elizabeth (Farquhar). 
Reference List of South African Noin-marine Mollusca. 239

505. Segmentina planodiscus, Melv. \& Pons. [S.A.M.] 1897 Planorbis (Segmentina) planodiscus, M. \& P., A.M.N.H. xix. 1898 p.638.pl. 17,f. 10. D.F.

Type in British Museum. M. \& P., Stur., S.A. Moll.

Hab. Natal. Umgeni Valley, near Durban (Burnup).

\section{Genus ANCYLUS, Geoffroy, 1767.}

(Coquilles de Paris, pp. 13, 122.)

Type of Genus, A. fluviatilis (Lin.).

Section BURnUPIA, Walker, 1912.

(Nautilus, xxv. p. 139.)

Type of Section, A. caffer, Krs.

506. Ancylus CAFFer, Krs.

1848 Ancylus caffer, Krs., Südafr. Moll. p. 70. pl. 4, f. 13. D.F.

1859 " $\quad$ gaulus, Gould, Proc. Boston Soc. Nat. Hist. vii.p.40. D.

$1862 \quad$ " , " , Otia Conch. p.106. D.

, $\quad$ " caffer, Krs., Bgt., Spicil. malac. p. 193. D.

1872 " caffra ", Sow., Conch. Icon. pl. 1, f. 5. D.F.

1882 " caffer ," Cless., Conch. Cab. p. 36. pl. 4, f.11. D.F.

Type of caffer in Stuttgart Museum; gaulus in U.S. Nat. Mus. Washington.

Hab. Natal. Pietermaritzburg (Krauss). Umkomaas; Tongaat; Equeefa River; Imputyni and Inkwalini streams near Pietermaritzburg (Burnup).

Cape of Good Hope (gaulus, Stimpson).

var. NANus, Walker.

1912 Ancylus caffer, Krs., var. nanus, Walker, Nautilus, xxv. p.139. D.

Type in coll. Walker.

Hab. Natal. Karkloof stream (Taynton). Pietermaritzburg (in coll. Ponsonby).

var. Gordonensis, Melv. \& Pons.

1903 Ancylus gordonensis, M.\&P., A.M.N.H.xii.p.606.pl.31, f.2. D.F. 1912 " caffer, Krs., var.gordonensis, M. \& P., Walker, Nautilus, xxv. p. 140, L, 
Type in British Museum.

Hab. NataL. Gordon Falls; Town Bush Valley and Sweetwater streams, near Pietermaritzburg; Edendale Falls; Nottingham Road; Dargle; Karkloof; Howick Falls and Aasvogel Krantz, Umgeni Kiver (Burnup).

Nomthern TransvaAl. Zoutpansberg (Cregoe).

var. FARQUHARI, Walker.

1912 Ancylus caffer, Krs.,var.farquhari, Walker, Nautilus,xxv.p.140. D. Type in coll. Walker.

Hab. Cape of Good Hope. York, East Griqualand (Farquhar). Teko River, Transkei (Miss Hickey).

var. stenochorias, Melv. \& Pons.

1855 Ancylus obliquus, Krs., Küst., Conch. Cab. pl. 1, f. 18-20. F. (non A. obliquus, Brod. \& Sow., 1832.)

1903 " stenochorias, M.\& P., A.M.N.H.xii.p.607.pl.31,f.1. D.F. 1912 " caffer, Krs., var. stenochorias, M. \& P., Walker, Nautilus, xxv. p. 140 . L.

Type in British Museum.

Hab. Cape of Good Hope. Ebb en Vloed, Bog Farm and Van Staadens River, Port Elizabeth (in coll. Ponsonby). Baakens River, Port Elizabeth; King Williamstown Road Dam and Kowie River, Grahamstown (Farquhar). Upper Retreat and Klipplatz River, Cathcart (Miss Hickey). Käser River, Montagu (Connolly).

var. CAPENsis, Walker.

1912 Ancylus caffer, Krs., var. capensis, Walker, Nautilus,xxv.p.141. D. Type in coll. Walker.

Hab. Cape of Good Hope. Lakeside and Hout Bay, Cape Peninsula (Connolly). Woost Hill and Blaauwkrantz Rivers, Grahamstown (Farquhar).

var. TRAPEzoIDEus, Böttger.

1897 ? Ancylus caffer, Krs., von Mits., D.-O.-Afr. p. 151. pl. 1, f. 19 $a, c, d . \quad$ D.F.

1907 Ancylus trapezoideus, Bttg. in Schultze, Aus Namaland u. Kalahari, p. 708. $N$.

1910

Abh. Senckenb. Naturf. Ges. Frankfurt, xxxii. p. 450. pl. 28, f. 15. D.F. 
1912 Ancylus caffer, Krs., var. trapezoideus, Bttg., Walker, Nautilus, xxv. p. 141. $L$.

Type in Senckenberg Mus. Frankfurt.

Hab. British Bechuanaland. Witkop (subfossil, Schultze).

Orange Free State. Valsch River, Lindley (Connolly).

Transvaal. Pretoria District (McBean). Zoutpansberg (Cregoe). Apparently also found in Central Africa.

\section{Ancylus mooiensis, Walker.}

1912 Ancylus mooiensis, Walker, Nautilus, xxv. p. 141. D.

Type in coll. Walker.

Hab. Transvaal, Mooi River, Potchefstroom (Miss Livingston).

var. Dubiosus, Walker.

1912 Ancylus mooiensis, var. dubiosus, Walker, Nautilus, xxv.p. 142. D.

Type in coll. Walker.

Hab. Transvaal. Pienaars Poort (Connolly).

Cape of Good Hope. Woost Hill River, Grahamstown (Farquhar).

508. Ancylus transvaalensis, Craven.

1880 Ancylus transvaalensis, Crvn., P.Z.S. p. 617. pl.57, f. 11. D.F. 1910 ? ", stenochorias, M. \& P., Bttg., Abh. Senckenb. Naturf. Ges. Frankfurt, xxxii.p. $450 . N$.

Type in British Museum.

Hab. Transvaal. Mooi River (Craven). Pretoria District (Connolly).

Orange Free State. Vereenigung (Johnson).

Cape of Good Hope. Prieska (in coll. Ponsonby).

? British Bechuanaland. Witkop (subfossil, Schultze).

509. Ancylus verreauxi, Bourguignat.

1853 Ancylus verreauxii, Bgt., Rev. et Mag. Zool. p. 351. D.

$1854 \quad, \quad$ ",$\quad$ pl. 1, f. 1-8. $F$.

$1856 \quad$ " $\quad$ " Amén. malac. i. p. 12. pl. 1, f. 1-8. D.F.

1862 " verreauxi " Spicil. malac. p. 194. N.

1882 " verreauxii ", Cless., Conch. Cab.p.60.pl. 5, f.4. D.F.

Type in Geneva Museum.

Hab. Cape Peninsula. "Ville de Constance" (? Constantia) (Verreaux). Retreat (Connolly). 


\section{Section FERRISSIA, Walker, 1903.}

(Nautilus, xvii. p. 15.)

Type of Section, A. rivularis, Say.

510. Ancylus Burnupi, Walker.

1912 Ancylus burnupi, Walker, Nautilus, xxv. p. 142. D.

Type in coll. Walker.

Hab. Natal. Equeefa (Burnup).

Cape of Good Hope. Brack Kloof, Grahamstown (Farquhar).

511. Ancylus connolimi, Walker.

1912 Ancylus connollyi, Walker, Nautilus, xxv. p. $143 . \quad D$.

Type in coll. Walker.

Hab. Cape of Good Hope. Black River, Maitland (Connolly). King Williamstown Road Dam, Grahamstown (Farquhar).

512. Ancylus equeefensis, Walker.

1912 Ancylus equeefensis, Walker, Nautilus, xxv. p. 143. D.

Type in coll. Walker.

Hab. Natal. Equeefa (Burnup).

513. Ancylus fontinalis, Walker.

1912 Ancylus fontinalis, Walker, Nautilus, xxv. p. 144. D.

Type in coll. Walker.

Hab. Transvaal. Ranjesfontein; Pienaars Poort (Connolly).

Orange Free State. Morgendal (Connolly).

514. Ancylus victoriensis, Walker.

1912 Ancylus victoriensis, Walker, Nautilus, xxv. p. 144. D.

'I'ype in coll. Walker.

Hab. Rhodesia. Victoria Falls (Connolly).

515. Ancylus zambesiensis, Walker.

1912 Ancylus zambesiensis, Walker, Nautilus, xxv. p. 144. D.

Type in coll. Walker.

Hab. Rhodesia. Victoria Falls (Connolly).

I am deeply indebted to Mr. Bryant IValker for the whole of the classification and distribution of this Genus. 


\section{Genus ISIDORA, Ehrenberg, 1831.}

(Symb. Phys., Evertebrata.)

Type of Genus, I. hemprichii, Ehrn. (contorta, Mich.).

Kobelt (1909) is probably correct in placing in this Genus all South African species formerly ascribed to Physa. Every one, whose animal has been examined, has proved to belong to Isidora, and it seems unlikely that Physa exists in the subcontinent.

The Genus has suffered severely for a long time from misidentification. It is the merest truism to remark that the speed of a stream and the nature of its soil have so great an effect on the shell of its molluscan inhabitants that colour, epidermal sculpture, and even substance or contour are of little account in the determination of fresh-water species; yet many have been identified, and others founded, on these features alone, without reference to the form of the parietal and columellar regions, which appear to offer the only moderately stable basis for comparison. I regret that the results of my investigation so far have been of a purely destructive nature, but fear that even more of this will be necessary before it is advisable to describe the one or two new species which possibly exist in South Africa. Mr. E. A. Smith has kindly assisted me in the examination of such species as are now, for the first time, brought into synonymy.

\section{IsIDORA ANGolensis (Morelet).}

1866 Physa angolensis, Morel., J. de C. xiv. p. 162. D.

1868 " " " Voy. Welwitsch, Moll. p. 88. pl. 9, f. 8. D.F.

1873 " algoensis „ Sow., Conch. Icon. pl. 7, f. 53. D.F. (Err. typ.)

1886 " angolensis " Cless., Conch. Cab. p. 334. pl. 47 (1885), f. 2. D.F.

Type in British Museum.

Hab. Damaraland (Geale).

Described from Angola.

Specimens of angolensis received by the British Museum from Geale in 1869 were incorrectly labelled algoensis, which gave rise to Sowerby's use of the latter name. Judging from the figure, I. parietalis (Mouss.), from Ovampoland, must be nearly allied to this species. It agrees in form, and the parietal plate, from which Mousson derived the name, is a frequent, and inconstant, feature in other members of the Genus. 
517. Isidora compta, Melv. \& Pons.

[S.A.M.]

1903 Isidora compta, M. \& P., A.M.N.H. xii. p. 606. pl. 32, f. 14. D.F.

Type in British Museum.

Hab. Transvant. Boksburg (MeBean, per Burnup).

Orange Free State. Kopjes; Morgendal; Valsch River, Lindley (Connolly).

Mashonaland, Enkeldoorn District (Miss Sharpe-Young).

Near I. tropica (Krs.), from which it can be distinguished by the curve of the outer lip, which may be called normal and regular in tropica, but in compta is usually distinctly flattened and almost incurved for a short way below the suture.

\section{Isidora contorta (Michaud).}

1829 Plyysa contortus, Mich., Bull. Soc. Linn. Bordeaux, iii. p. 268. plate, f. 15, 16. D.F.

1831 " contorta, Mich., Complément Hist. Moll. Fir. p. 83.

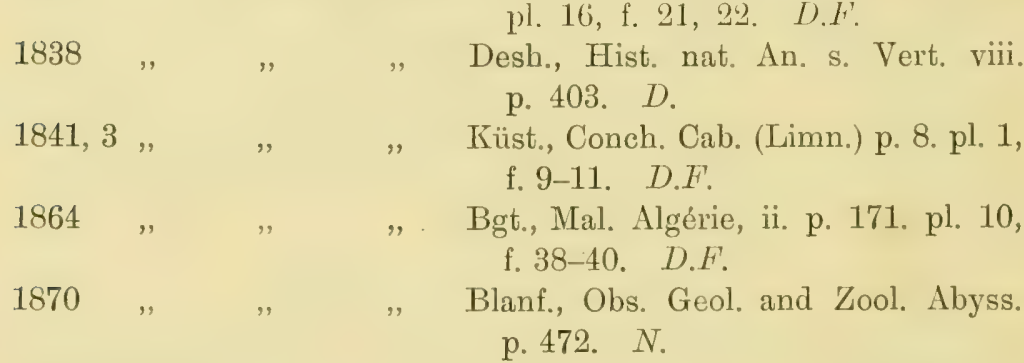

1874 Isidora ", " Jick. (=tropica, Krs., and cyrtonota, Bgt.), Fauna N.-O.-Afr. p. 203.pl. 3, f. 4. pl. 7, f. 14 . D.R.F. " Physa contorta, Mich., Sow., Conch. Icon. pl. 11, f. 92 . D.F. $1886 \quad$ " $\quad$ " $\quad$, Cless., Conch. Cab. p. 314. D.N. 1908 Isidora ", ", (=sericina, Jick.), Neuville \& Anthony, Ann. Sci. nat. Paris, viii. pp. 274, 275. N.F. Type-ubi?

Hab. Cape of Good Hope. Quarry near Fort England, Grahamstown (Farquhar).

A set of hardly mature shells from the above locality appear inseparable from Michaud's variable species, which is widely diffused through the South of Europe and North Africa, and recorded from West Africa and the Euphrates. Jickeli (1874) was very possibly right in placing tropica in the synonymy; but I have so far been unable to confirm his views, and as the last-named species is, typically, a well-marked one in South Africa, with a 
special synonymy of its own, it appears advisable to allow it to maintain an individual existence until further material is available for examination.

Jickeli further included, under contorta; truncata and harpula, Fér.; brocchii and hemprichi, Ehrn.; rivularis, Phil.; mareotica and spiracea, Parr. in coll.; pyrum, Poro; and scalata, Merian.

\section{IsIdora diaphana (Krauss).}

1848 Physa diaphana, Krs., Südafr. Moll. p. 84. pl. 5, f. 11. D.F.

1856 ", , Bgt., Rev. et Mag. Zool. viii. p. 236. D.

\begin{tabular}{|c|c|c|}
\hline & $"$ & " Amén. malac. i. p. $175 . \quad D$. \\
\hline 866 & " & on Mts., Mal. Blätt. xiii. p. $100 . N$. \\
\hline 873 & & Sow., Conch. Icon. pl. 10, f. 81. D.F. \\
\hline & & Jonch. Cab.p.282 \\
\hline
\end{tabular}

Type in Stuttgart Museum.

Hab. Natal. Umgeni Valley (Krauss).

520. Isidora ForskaLi, Ehrenberg.

[S.A.M.]

1831 Isidora forstalii, Ehrn., Symb. Phys., Evert. 3rd Sp. D.

1848 Physa wahlbergi, Krs., Südafr. Moll. p. 84. pl. 5, f. 13. D.F.

1856 " " " Bgt., Rev. et Mag. Zool. viii. p. 240. D.

" " forskalii, Ehrn. " ", viii. p. 235. D.

, " " $\quad$ " $\quad$, Amén. malac. i. p. 174. D.

" "wahlbergi, Krs. " " i. p. $180 . \quad D$.

$1863 \quad$ " , , " Küst., Conch. Cab. (Limn.) p. 71. pl. 12, f. $23,24 . \quad D . F$.

$1866 \quad$ " $\quad$, $\quad$, von Mts., Mal. Blätt. xiii. p. 100. N.

$1869 \quad$ " $\quad$ " $\quad$ Dohrn, Mal. Blätt. xvi. p. 15. N.

, " (Isidora) forskalii,Ehrn., von Mts., Mal.Blätt.xvi.p.213. N.

1873 ", walbergii, Sow., Conch. Icon. pl. 8, f. 58. D.F.

1874 Isidora forskali, Ehrn., Jick., Fauna N.-O.-Afr. p. 198. pl. 3, f. 3. pl. 7, f. 13. D.R.F. and Synonymy.

1886 Physa forskalii, Ehrn., Cless., Conch. Cab. p. 320. pl. 39 (1884), f. 2. D.F.

1897 Isidora forskali, Ehrn., von Mts., D.-O.-Afr.p. 141.pl.1,f.15. D.F. 1898 Physa gradata, M. \& P., A.M.N.H. ii. p. 129. pl. 7, f. 8. D.F. 1899 Isidora forskali, Ehrn. (=gradata), M.\&P.,A.M.N.H.iv.p.193. N. 1908 "Neuville \& Anthony, Ann. Sci. nat. Paris, viii. pp. 271-273. N.F.

Type of forskall in Zool. Mus. Berlin; wahlbergi in Stuttgart Museum; gradata in British Museum. 
Hab. Transvaal. River Limpopo (wahlbergi, Wablberg). Pretoria (Farquhar).

Cape of Good Hope. Brickfields, Grahamstown (gradata, Farquhar).

Natal Pietermaritzburg; Durban; Thornville (Burnup).

Also said to be found in North and East Africa, Aden, Cape Verde Islands, Angola and Benguela.

Jickeli (1874) includes in the synonymy wahlbergi, Kirs. ; lamellosa, Roth; scalaris and schmidti, Dkr.; fischeriana, Bgt.; capillacea, clavulata, semiplicata, turriculata and apiculata, Morelet; beccarii, Paladilhe; vitrea, Parr. ; and jickelii, Krs.

521. Isidora NATALENSis (Krauss).

1841, 43 Physa natalensis, Krs., Küst., Conch. Cab. (Limn.) p. 8. pl. 1, f. 12-14. D.F.

1848 Physa natalensis, Krs., Südafr. Moll. p. 84. pl. 5, f. 10. D.F. $1856 \quad$ ", Bgt., Rev. et Mag. Zool. viii. p. 237. D. " $\quad$ " $\quad$ " Amén. malac. i. p. 176. D.

$1869 \quad$ ", von Mts., Mal. Blätt. xvi. p. 214. N.

$1873 \quad$ " $\quad$ "Sow., Conch. Icon. pl. 10, f. 79. D.F.

1874 Isidora natalensis , Jick., Fauna N.-O.-Afr. p. 196. N.

1883 Physa natalica, Bgt., Ann. Sci. nat. Paris, xv. pp. 98, $126 . \quad N$. 1903 „, zuluensis, M. \& P., A.M.N.H. xii. p. 606. pl. 32, f. 4. D.F. 1910 Isidora natalensis, Krs., Bttg., Abh. Senckenb. Naturf. Ges. Erankfurt, xxxii. p. 453. N.

Type of natalensis in Stuttgart Museum; zuluensis in British Museum.

Hab. Natal. Umgeni Valley (Krauss). Durban (Burnup).

East Zululand (zuluensis, Burnup). Lake Sibayi (Toppin).

Lorenzo Marques. Rikatla and Monguane Lakes (Junod).

British Bechuanaland. Witkop (subfossil, Schultze).

Taking into consideration the great range of variation to be found in South African fresh-water shells, it appears impossible to separate zuluensis, which was likened by its joint authors to natalensis, from the last-named species.

522. Isidora parietalis (Mousson).

1887 Physa parietalis, Mouss., J. de C. xxxv. p. 298. pl. 12, f. 8. D.H' 1904 von Mts., Die Kalahari, p. 756. N. 1910 Isidora " " , Bttg., Abh. Senckenb. Naturf. Ges. Frankfurt, xxxii. p. 452. N. 
Type in Zurich Museum.

Hab. Ovampoland. Ondonga (Schinz).

Damaraland. Okosongoho (Hermann).

Bechuanaland. Lake Ngami; Garu; fossil, south of Hardeko Drift, Botletle River (Passarge).

British Bechuanaland. Witkop (subfossil, Schultze).

? CAPE of Good Hope. Port Elizabeth (fide Morelet).

Considered by Böttger to be very near diaphana; Mousson's figure, however, closely resembles angolensis, which is known from Angola and Damaraland.

\section{ISIDORA SERICINA, Jickeli.}

1874 Isidora sericina, Jick., Fauna N.-O.-Afr. p. 194. pl. 3, f. 2. pl. 7, f. 11. D.R.F.

1886 Physa " $\quad$ " Cless., Conch. Cab. p. 325. pl. 39 (1884), f. 12. D.F.

$1898 \quad " \quad$ " $\quad$ " M. \& P., Proc. Mal. Soc. iii. p. 182. L. " schackoi " " " " " 1908 Isidora sericina ", Neuville \& Anthony, Ann. Sci. nat. Paris, viii. pp. 274, 275. N.F.

Type in Zool. Mus. Berlin.

Hab. "South Africa" (in coll. Ponsonby).

CAPE OF Good Hope. Forty-eight miles from Port Elizabeth ("schackoi," in coll. Ponsonby).

Described from Abyssinia.

Careful examination of the two large series, on which the record of sericina and schackoi from South Africa was originally based, proves them to contain only one species. Professor Thiele has kindly compared this with Jickeli's types, and informs me that it is distinct from schackoi, but nearly allied to sericina, with which it may be identical. I have therefore removed schackoi from the South African list. Neuville and Anthony place sericina in the synonymy of contorta, Mich. The shells are certainly very similar, and might merge into one another, but Jickeli differentiated the two species by their radula, and, under these circumstances, it may be inadvisable to unite them without more extended investigation.

524. Isidora tropica (Krauss).

1848 Physa tropica, Krs., Südafr. Moll. p. 84. pl. 5, f. 12. D.F. $1856 \quad " \quad$ " " Bgt., Rev. et Mag. Zool, viii. p. 236. D. " " " " $\quad$ Amén. malac. i. p. 175. $D$. 
1856 Physa cyrtonota, Bgt., Rev. et Mag. Zool. viii. p. 238. pl. 15, f. 1,2 . D.F.

, $\quad$ " $\quad$ " Amén. malac. i. p. 177. pl. 21, f. 1,2. D.F. 1873 ", tropica, Krs., Sow., Conch. Icon. pl. 5, f. 32 . D.F.

1874 Isidora " " Jick., Fauna N.-O.-Afr. p. 205. N. 1880 Physa lirata, Crvn., P.Z.S. p. 617. pl. 57, f. 10. D.F.

1885 ", tropica, Krs., Cless., Conch. Cab.p.288.pl.41, f.8,11. D.F. 1886 " cyrtonata, Bgt., Cless., ibid.p.323. pl.39(1884), f.5. D.F. " . craveni, Ancey (=lirata, Crvn., 1880, nec Tristram, 1863), Ancey, Le Naturaliste, viii. p. 358.

1889 " lirata, Crawf., Morel., J. de C. xxxvii. p.20. (Em.typ.)

1897 Isidora tropica, Krs., von Mts., D.-O.-Afr., p. 140. D.

1898 Physa craveni, Stur., S.A. Moll. p. 76. Emend. Nom.

1899 " $\quad$ Ancey, M. \& P., A.M.N.H. iv. p. 193. N.

1910 Isidora tropica, Krs., Bttg., Abh. Senckenb. Naturf. Ges. Frankfurt, xxxii. p. $453 . N$.

Type of tropica in Stuttgart Museum; cyrtonota in Geneva Museum; lirata in British Museum.

Hab. Transvaal. River Lepenula (tropica, Wahlberg). Mooi River (lirata, Craven).

Natal. Examples recorded, but require verification.

CAPE OF GOOD Hope. Port Elizabeth (lirata, fide Morelet). Stellenbosch (Péringuey). Montagu; Black River, Maitland (Connolly). Olifant River (cyrtonota, Verreaux).

Damaramand. Okaputa Pan (subfossil, Hermann).

Jickeli remarks that cyrtonota "agrees perfectly with tropica." I have not seen an authentic example of Bourguignat's species, but judging from his description and figure, it certainly does so. The type set of lirata are slightly immature specimens of tropica, of which there is a fine co-type available for comparison in the British Museum. Krauss mentions that the young of tropica have a ribbed epidermis, the feature on which Craven founded his species.

525. IsIdORA verreauxi (Bourguignat).

1856 Physa verreauxii, Bgt., Rev. et. Mag. Zool. viii. p. 237. pl. 15, f. 3,4 . D.F.

" $\quad$ " $\quad$ Amén. malac. i.p. 176.pl. 21, f.3,4. D.F.

$1886 \quad$," $\quad$ ", Cless., Conch. Cab. p. 317. pl.39(1884), f. 1. D.F.

Type-ubi?

Hab. Cape of Good Hope. Olifant River; Knysna (Verreaux). 
526. Isidora zanzibarica (Clessin).

[S.A.M.]

1886 Physa zanzebarica, Cless., Conch. Cab. p. 362. pl. 51, f. 5. D.F. 1889 " cornea, Morel., J. de C. xxxvii. p. 16. pl. 1, f. 8. D.F. 1897 Isidora zanzibarica, Cless., von Mts., D.-O.-Afr. p. 140. N.

Type of zanzibarica in Zool. Mus. Berlin; co-types of cornea in British Museum.

Hab. CaPe of Good Hope. Port Elizabeth (Crawford).

von Martens infers that the loc. Zanzibar, originally quoted for zanzibarica by Clessin, is incorrect, and that the species is the same as that which was subsequently described by Morelet as cornea. It is nearly allied to contorta.

I. zanzibarica has recently been recorded by Thiele from Central Africa.

\section{Genus PHYSOPSIS, Krauss, 1848.}

(Südafr. Moll. p. 85.)

Type of Genus, Ph. africana, Krs.

527. Physopsis africana, Krauss.

[S.A.M.]

1848 Physopsis africana, Krs., Südafr. Moll. p. 85. pl. 5, f. 14. D.F. 1855 " H. \& A. Ad., Gen. rec. Moll. iii. pl. 83, f. $10 . \quad F$.

1856 ", Bgt., Rev. et Mag. Zool. viii. p. 241. D.

" " " " Amén. malac. i. p. 180. D.

1863 " Küst., Conch. Cab. p. 72. pl.12, f. 29, 30. D.F.

1866 " " von Mts., Mal. Blätt. xiii. p. 8. N.

1874 Physa africana, Krs., Sow., Conch. Icon. pl. 1, f. 3. D.F.

", Physopsis africana, Krs., Jick., Fauna N.-O.-Afr. p. 209. D. 1879

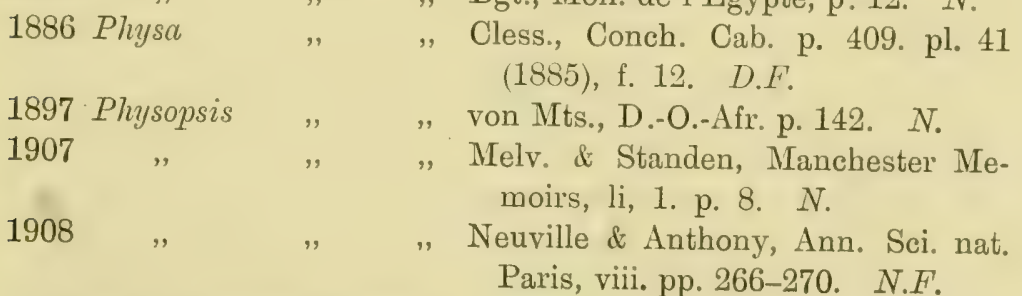

" Bgt., Moll. de l'Égypte, p. 12. N.

Type in Stuttgart Museum.

Hab. NataL. Port Natal (Wahlberg). Pietermaritzburg; Pinetown; Lower Umkomaas (Burnup).

Zululand (Burnup; Toppin).

Transvaal, Pretoria District (Connolly). Middelburg (Crawford). 
Rhodesis. Gwelo (Dodds). Enkeldoorn District, Mashonaland (Miss Sharpe-Young).

Lorenzo Marques. River Zambesi (Peters; Kirk; Penther).

Cape of Good Hope. Port Elizabeth(Crawford). Knysna (Purcell).

Also distributed up the eastern side of the Continent.

Melvill and Standen, in chronicling this species from Northern Rhodesia, remark: "Our specimens seem intermediate between the type and ovoidea, Bgt., which surely can be but a variety." Jickeli places werneana, Troschel, in the synonymy of africana, and expresses doubt whether "globularis, Morel." (globosa, Morel., Voy. Welwitsch, p. 93. pl. 9, f. 6, and J. de C., 1866, p. 162), is separable therefrom, while Neuville and Anthony consider that abyssinica, von Mts., and eximia, Bgt., as well as ovoidea, Bgt., merge into Krauss' species.

\section{Sub-Class STREPTONEURA, Spengel, 1881.}

(Zeitschr. f. wissens. Zool. Leipzic, p. 372.)

ORdER PECTINIBRANCHIA, Cuvier, 1817.

(Règne Animal, ii. pp. 388, 415, as Pectinibranches.)

Sub-Order T ENIOGLOSSA, Troschel, 1847.

(Arch. f. Naturg. xiii, 2. p. 382, and Wiegmann's Handbuch der Zool,

Ed. 3. 1848.)

Tribe PLATYPODA, Fischer, 1883.

(Man. de Conch. p. 445.)

FAMrLY CYCLOPHORIDAE, Gray, 1847.

(P.Z.S. p. 181.)

Sub-FAmily CYCLOPHORIN A, Fischer, 1885.

(Man. de Conch. p. 739.)

Genus CYCLOPHORUS, de Montfort, 1810.

(Conch. Syst. ii. p. 290.)

Type of Genus, C. volvulus (Müll.).

Sub-Genus MAIZANIA, Bourguignat, 1889.

(Moll. de l'Afr. équat. p. 148.)

(=Afernlus, von Mts., 1897 ; Natalia, G.-Aust., 1897; Hijabia, G.Aust., 1898; Austrocyclus, Ancey, 1898; and Cyclophoropsis, Dautzenberg, 1908.)

Type of Sub-Genus, C. olivaceus, Bgt. 
528. Cyclophorus (Maizania) wahlbergi (Benson), [S.A.M.] 1848 Cyclostoma transhucidum, "Sow.," Krs., Südafr. Moll. p. 83. N. 1852 " wahlbergi, Bs., A.M.N.H. x. p. 271 . D.

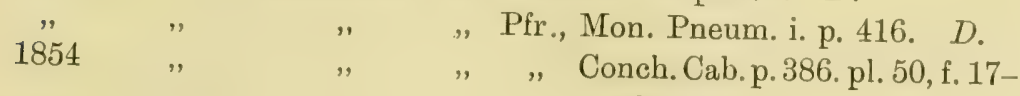

\section{Cyclophorus}

1881

1902

,$\quad(11$,

$$
\text { 19. D.F. }
$$

,, Rve., Conch. Icon. pl. 17, f. 81. D.F. ", ", Smith, P.Z.S. p. 277. N.

(Maizania) wahlbergi, Bs., Kob., Tierreich, xvi. pp. 148, 152. F.D.

Specimens ex auct. in University Museum of Zoology, Cambridge.

Hab. Natal (Wahlberg, per Stevens). Durban; Isipingo (Penther). Generally in the bush fringing the coast (Burnup).

Cape of Good Hope. Pondoland (Beyrich). Port Grosvenor (Bachmann).

Also known in German East Africa and Nyassaland.

Smith (1881) considers that C. magilensis, Craven, from Magila, may prove to be only the young state of this species.

\section{Genus CHONDROCYCLUS, Ancey, 1898. \\ (Bull. Mus. Marseille, i. p. 136.)}

Type of Genus, C. convexiusculus (Pfr.).

529. Chondrocyclus convexiusculus (Pfeiffer). [S.A.M.]

1855 Cyclostoma (Cyclophomu) convexiusculum, Pfr., P.Z.S. p. 104. D. 1858 Cyclophorus convexiusculus, Pfr., Mon. Pneum. ii. p. 68. D. 1861

$$
\begin{aligned}
& \text { " Rve., Conch. Icon. pl. 19, f. } 92 . \\
& \text { D.F. }
\end{aligned}
$$

1880 Cyclotus alabastris, Craven, P.Z.S. p. 619. pl. 57, f. 9. D.F. 1899 Chondrocyclus alabastris, Crvn., M. \& P., A.M.N.H.iv.p. 193. N. 1902

" Cyclophorus (Maizania) alabastris, Crvn., Kob., ibid. p. 149. D. Types in British Museum.

Hab. Cape of Good Hope (Macgilliviay). George District; Tharfield; Camps Bay; Simonstown (Layard). Montagu (Connolly). Port Elizabeth (Crawford). Grabamstown; Bedford (Farquhar). Cape Récif, Algoa Bay (alabastris, Craven). Pirie Forest (Godfrey).

As Crawford writes, alabastris is a beach-rolled and polished specimen of convexiusculus, in the synonymy of which it must be placed. 
var. MrNor, Benson.

Cyclophorus plicicutis, Bs., in litt.

1856 ", convexiusculus, Pfr., var. minor, Bs., A.M.N.H. xviii. p. 438 . D.

1858 ". convexiusculus, Pfr., var. minor, Bs., Pfr., Mon. Pneum. ii. p. $68 . \quad D$.

Hab. Cape Peninsula. Table Mountain (Layard).

The form most commonly found in the Cape Peninsula, but hardly worthy of varietal rank.

530. Chondrocyclus exsertus, Melv. \& Pons. [S.A.M.]

1903 Chondrocychus exsertus, M. \& P., A.M.N.H. xii. p. 608. pl. 32, f. $11 . D . F$.

Type in British Museum.

Hab. Natal. Umkomaas; Umbogintwini; Equeefa (Burnup).

531. Chondrocyclus isipingöensis (Sturany). [S.A.M.] 1898 ? Cyclotus isipingöensis, Stur., Anz. k. Akad. Wiss. Wien. xvi. Sonderabdruck, p. $9 . \quad D$.

" Cyclophorus minimus, M. \& P., A.M.N.H.ii.p. 129.pl.7, f.9. D.F. " ?Cyclotus isipingöensis, Stur., S.A. Moll. p. 81.pl. 2, f. 37-39. D.F. 1899 Cyclophorusminimus,M.\&P., A.M.N.H.iv.pl.3, f. 15 (Operculum). 1902 ," (Maizania) isipingöensis, Stur., Kob., Tierreich, xvi. p. 149 . D.

minimus, M. \& P., Kob., ibid. p. 150. D.

"Type of minimus in British Museum; isipingöensis in Naturh. Hofmus. Vienna.

Hab. Natal. Durban; Isipingo (Penther). Pietermaritzburg, and in bush generally, from the coast to Dargle and Karkloof (Burnup).

Cape of Good Hope. Maeström Forest, Bedford (Farquhar).

It seems more natural to place this species in the same Genus as convexiusculus, which it resembles in miniature, than in Maizania.

FAMily POMATIIDE, B. B. Woodward, 1903.

(Journ. of Conch. x. p. 356.)

(=Cyclostomatidæ, Auctt.)

Genus TROPIDOPHORA, Troschel, 1847.

(Zeitschr. f. Malak. iv, p. 44.)

Type of Genus, T. cuvieriana (Petit). 
Sub-Genus LigATELLA, von Martens, 1880.

(Meeresfauna Mauritius, p. 186.)

(=Rochebrunia, Bourguignat, 1881.)

Type of Sub-Genus, T. listeri (Gray).

532. Tropidophora calcarea (Sowerby).

1822 Cyclostoma sulcata, Lam., Hist. nat. An. s. Vert. vi, 2. p. 144. D. 1838:

"Desh., Hist. nat. An. s. Vert. viii. $1841^{+},, \quad, \quad$, p. 354 . $D$.

Delessert, Recueil de Coquilles décrites par Lamarck, pl.29, f 9. D.F.

1847 ," calcareum, Sow. (=sulcata, Lam., 1822, nec Drap., 1805), Sow., Thesaurus Conch. i. p. 118. pl. 26, f. 113. D.F.

1848 Cyclostoma calcareum, Sow., Pfr., Conch. Cab. p. 88. pl. 11 (1847), f. 11, 12. D.F.

1852 Cyclostomus calcareus, Sow., Pfr., Mon. Pneum. i. p. 201. D.

1858 "sulcatus, Lam. (=calcareus, Sow.), Pfr., Mon. Pneum. ii. p. 115.

1861 Cyclostoma calcareum, Sow., Rve., Conch. Icon. pl. 3, f. 13. D.F'. 1879 ", Gibb., Journ. of Conch. ii.p. 145. N. 1881 ", insulare (var.), Smith, P.Z.S. p. 277.pl. 32, f. 1. N.F'. 1897 „, calcareum, Sow., von Mts., D.-O.-Afr. p. 3. N.

Originals of calcarea in British Museum.

Hab. Lorenzo Marques. Tette (Kirk; Thomson).

Also known from East and Central Africa.

533. Tropidophora comburens, Melv. \& Pons. [S.A.M.] 1903 Tropidophora comburens, M. \& P., A.M.N.H. xii. p. 608. pl. 32, f. $12 . D . F$.

Type in British Museum.

Hab. Zululand. Makowe (Burnup).

534. Tropidophora foveolata (Melv. \& Pons.).

1895 Cyclostoma foveolatum, M. \& P., A.M.N.H.xv.p.164.pl.12,f.4. D.F. Type in British Museum.

Hab. "S. Africa" (fide M. \& P.).

Cape of Good Hope. Port Alfred (Farquhar).

? Natal (fide Sturany). 
535. Tropidophora hartvigiana (Pfeiffer).

1862 Cyclostomus hartvigianus, Pfr., Mal. Blätt. ix. p. 203. D.

1863

1865
, Novit. Conch. ii. p. 225. pl. 59, f. 1, 2. D.F.

, Mon. Pneum. iii. p. 131. D.

Originals in British Museum.

Hab. "South Africa" (Hartvig).

536. Tropidophora insularis (Pfeiffer).

1852 Cyclostoma insulare, Pfr., P.Z.S. p. 64. D.

Cyclostomus insularis, Pfr., Mon. Pneum. i. p. $215 . \quad D$.

1854 Cyclostoma insulare, Pfr., Conch. Cab. p. 351.pl. 45, f. 5, 6. D.F. $1861 \quad$ ", $\quad$, Rve., Conch. Icon. pl. 8, f. $41 . \quad$ D.F. " $\quad$ " kraussianum, Pfr., Rve., Conch.Icon. pl.9, f.52. D.F. 1897 ", insulare, Pfr., von Mts., D.-O.-Afr. p. 5. N.

The species figured by E. A. Smith (P.Z.S., 1881, p. 277. pl. 32,

f. 1) is attributed by von Martens to calcarea.

Type in British Museum.

Hab. Natal. Generally, on the coast and in the Midlands (Burnup).

Transvaal. Pietpotgietersrust (Connolly).

Griqualand West. Modder River, near. Kimberley (Miss Wilman).

Cape of Good Hope. Alexandria District (Crawford).

Originally described as from Mauritius.

537. Tropidophora kraussiana, Pfeiffer.

1852 Cyclostoma (Tropidophora) kraussianum, Pfr., P.Z.S. p. 64. D.

"Cyclostomus kraussianus, Pfr., Mon. Pneum. i. p. 204. D.

1854 Cyclostoma kraussianum „, Conch. Cab. p. 334. pl. 43, f. 17, 18. D.F.

$1881 \quad$ " $\quad$, Smith, P.Z.S. p. 278. N.

$1897 \quad$ " $\quad$ "von Mts., D.-O.-Afr. p. 5. N.

Type in British Museum.

Hab. Natal. Cape Natal (coll. Cuming).

Transvaal。 Pilgrim's Rest (Craven).

Lorenzo Marques. Inhambane (Gibbons).

Cape of Good Hope. Port Elizabeth (fide Morelet). Pondoland (Beyrich), and Port Grosvenor (Bachmann), fide von Martens.

The type, from the Cuming collection, appears to be a deformed shell, and the other localities quoted should be accepted with due reservation. 
538. Tropidophora ligata (Müller).

[S.A.M.]

1774 Nerita ligata, Müll., Verm. ii. p. 181. D.

1786 Turbo ligatus, Müll., Chem., Conch. Cab. ix, 2. p. 60. pl. 123, f. 1071,$1072 ; 3,4$. D.F.

1822 Cyclostomaligata, Müll., Lam., Hist. nat. An.s.Vert.vi,2.p.147. D. 1831 ", ligatum, Müll., Sow., Genera of Shells, pl.176,f.4. F. 1838 " ligata, Lam., Desh., Hist. nat. An. s. Vert. viii. p. $359 . \quad D . N$.

1847 „, ligatum, Müll., Pfr., Conch. Cab. p. 33. pl. 4 (1843), f. 12,13 . pl. 8 , f. 3,4 . D.F.

Lam., Sow., Thesaurus Conch. i. p. 98. pl. 23, f. 24. D.F.

$1848 \quad$ " $\quad$ ", Lam., Krs., Südafr. Moll. p. 82. D.N.

1852 Cyclostomus ligatus, Müll, Pfr., Mon. Pneum. i. p. 221. D.

1859 Cyclostoma ligatum ", von Mts., Mal. Blätt. vi. p. 215. N. 1861

1897 " Rve., Conch. Icon. pl. 9, f. 54. D.F. , von Mts., D.-O.-Afr. p. 5. N.

Original in University Zool. Mus. Copenhagen.

Hab. Cape of Good Hope. Simon's Bay (McGillivray). Port Elizabeth ; Alexandria District; Uitenbage; Addo (Crawford). Tharfield (Layard). Mossel Bay; Port Alfred (Farquhar). Knysna(O'Connor).

NatAL. Coast and Midlands, generally (Burnup). Kamiesberg (Zeyher).

Transvaal. Lydenburg; Barberton (fide Sturany).

Lorenzo Marques. Tette (Peters). Rikatla (Junod).

var. CAFFRA, Beck.

1861 Cyclostoma caffrum, Beck, Rve., Conch. Icon. pl.11, f. 67. D.F. 1865 Cyclostomus caffer ", Pfr., Mon. Pneum. iii. p. 129. D.

Originals in British Museum.

Hab. Cape of Good Hope (Ecklon, in British Museum).

Merely a white form of ligata.

var. MINOR, Pfeiffer.

[S.A.M.]

1828 Turbo ligatus, Wood, Index Test. p. 151. pl. 32, f. 122. F.

1847 Cyclostoma affine, Sow. (=Turbo ligatus, Wood), Sow., Thesaurus Conch. i. p. 98. pl. 23, f. 25, 26. D.F.

1848 Cyclostoma affine, Sow., Pfr., Conch. Cab. p. 62 . pl. 8 (1847),

f. 17,18 . D.F.

$" \quad$ " Krs., Südafr. Moll. p. 82, N. 
1852 Cyclostomus ligatus, Müll., var. minor, Pfr. (=C. affine, Sow.) Pfr., Mon. Pneum. i. p. $222 . \quad N$.

Hab. Cape Peninsula. Generally distributed.

539. Tropidophora lineata (Pfeiffer).

1852 Cyclostoma lineatum, Pfr., P.Z.S. p. 65. D.

" Cyclostomus lineatus " Mon. Pneum. i. p. $222 . \quad D$.

1854 Cyclostoma lineatum ", Conch. Cab. p. 350.pl.45, f.3,4. D.F. $18 \$ 1 \quad$ ", Smith, P.Z.S. p. 278. N.

Type in British Museum, from "? Madagascar. Coll. Cuming."

Hab. Natal (coll. Cuming, in British Museum).

540. Tropidophora ochracea (Melv. \& Pons.).

1896 Cyclostoma ochraceum, M. \& P., A.M.N.H. xviii. p. 318. pl. 16, f. $8,9 . \quad$ D. F .

Type in British Museum.

Hab. "South Africa" (fide M. \& P.).

541. Tropidophora plurilirata, Fulton.

1903 Tropidophora plurilirata, Fulton, Journ. of Malac. x. p. 103. pl. 9, f. 8. D.F.

Type in British Museum.

Hab. Cape of Good Hope. Grahamstown (Miss Leppan; Farquhar).

Natal. Pinetown; Pietermaritzburg (Burnup).

542. Tropidophora sarcodes (Pfeiffer).

1856 Cuclostoma sarcodes, Pfr., P.Z.S. p. 339. D.

1858 Cyclostomus ", " Mon. Pneum. ii. p. 120. D.

1861 Cyclostoma ", " Rve., Conch. Lcon. pl. 10, f.61. D.F.

Type in British Museum.

Hab. Cape of Good Hope. Knysna (Cox; O’Connor).

The type-locality-Madagascar (coll. Cuming) - is probably erroneous.

543. Tropidophora transvaalensis (Melv. \& Pons.). [S.A.M.] 1891 Cyclostoma transvaalense, M. \& P., A.M.N.H. viii. p. 237. D. 1892 ix. p. 94 . pl. 5 , f. 6 . N.F.

Type in British Museum.

Hab. Transvala. Pretoria (Farquhar). 
FAMily AMPULLARIIDE, Gray, 1840.

(Syn. Brit. Mus. p. 115, as Ampullariadæ, cmend. d'Orbigny, 1842, Paléont. Franç., Terr. Crétrcés, ii. p. 30.)

Genus AMPULLARIA, Lamarck, 1799.

(Mém. Soc. Hist. Nat. Paris, p. 76.)

Type of Genus, A. ampullacea (Lin.).

544. Ampullaria occidentalis, Mousson.

1887 Ampullaria occidentalis,Mouss., J.de C.xxxv.p.299.pl.12,f.9. D.F. $1904 \quad$ " $\quad$ von Mts.,Die Kalahari,p.756. $N$. $1910 \quad$ " $\quad$ "Bttg., Abh. Senckenb. Naturf.

Type in Zurich Museum. Ges.Frankfurt,xxxii.p.453. $N$.

Hab. Ovampoland. Kunene River (Geale; Schinz).

Damaraland. Okosongoho (Hermann).

Bechuanaland. Nausche, or Nausib, River; Okavango marshes; Lake Ngami; Botletle District (Passarge).

Also chronicled from Angola.

Genus Lanistes, de Montfort, 1810. (Coneh. Syst. ii. p. 122.)

Type of Genus, $L$. olivieri, de Montf.

Sub-Genus MELAdomus, Swainson, 1840.

(Treatise on Malac. p. 340.)

Type of Sub-Genus, L. bulimoides, Swains. (purpureus, Jonas).

545. Lanistes ellipticus, von Martens.

1866 Lanistes ellipticus, von Mts., Novit. Conch. ii. p. 294. pl. 70, f. 9,10 . D.F.

1877 „ solidus, Smith, P.Z.S. p. 716. pl. 74, f. 10, 11 . D.F. 1886 " zambesianus, Furtado, J.de C.xxxiv.p.148.pl.7,f.1. D.F. 1897 ", ellipticus, von Mts. (= solidus, Smith), von Mts., D.-O.-Afr. p. $168 . \quad N$.

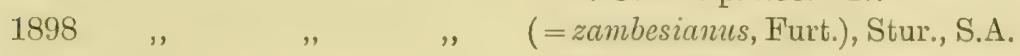
Moll. p. 87. N.

Type of ellipticus in Zool. Mus. Berlin; solidus in British Museum. Hab. Lorenzo Marques. Tette (Peters; Ivens; Capello).

Rhodesia. Mouth of Tschobe River (Holub). 
L. solidus was recorded from Quilimane and Nyassa, and zambesianus from below Tette.

var. Trapeziformis, Furtado.

1886 Lanistes ellipticus, von Mts., var. trapeziformis, Furtado, J. de C. xxxiv. p. 150. D.

Hab. Lorenzo Mirques. River Zambesi, below Tette (Capello \& Ivens).

546. Lanistes olivaceus, Sowerby, var. ambiguus, von Martens. 1851, 2 Ampullaria ovum (pars), Phil,, Conch. Cab.p. 22. pl. 7, f.7. D.F. 1866 Lanistes olivaceus, Sow., var. ambiguus, von Mts., Novit. Conch. ii. p. 292. pl. 71, f. 3, 4. D.F.

1879 Meladomus ambiguns, von Mts., Bgt., Moll. de l'Égypte, p. 34. 1897 Lanistes olivaceus, Sow., var. ambiguns, von Mts., D.-O.-Afr. p. 165 . D.N.

Type in Zool. Mus. Berlin.

Hab. Lorenzo Marques. Tette (Peters). Delagoa Bay (Junod; native name, Chibyabya). Gorongozo District (Wells-Cole).

\section{Lanistes ovum, Peters.}

1845 Lanistes ovum, Ptrs., Trosch., Archiv. f. Naturg. xi, 1. p. 215. D. 1851, 2 Ampullaria ovum, Ptrs., Phil., Conch. Cab. p. 22. pl. 6, f. 2. $F$. 1857 Lanistes ovum, Ptrs., Trosch., Gebiss der Schnecken, i. p. 90. pl. 6, f. 11 . $R$.

\begin{tabular}{|c|c|c|}
\hline 1866 & $"$ & von Mts., Novit. Conch. ii. p. 290. N. \\
\hline 1870 & , & , Mal. Blätt. xvii. p. 35. N. \\
\hline 874 & , & Jick., Fauna N.-O.-Afr. p. 230. D. \\
\hline 877 & affinis, & Smith, P.Z.S. p. 716. pl. 74, f. 7. D.F. \\
\hline 881 & , & , p. 290. pl. 34, f. $23 . \quad$ N.F. \\
\hline 9. & " & $\begin{array}{l}\text { von Mts., Sitz.-Ber. Ges. Nat. Fr. } \\
\text { Berlin, p. } 17 . \quad N .\end{array}$ \\
\hline 97 & ovum, & $\begin{aligned} \text { Ptrs. } & =\text { affinis, Smith), von Mts., D.-O.-Afr. } \\
& \text { p. } 166 . \text { D.N. }\end{aligned}$ \\
\hline 398 & ", & Stur., S.A. Moll.p. 86.pl.3, f.62,63. N.F' \\
\hline 07 & " & von Mts., Die Kalahari, p. 756. $N$. \\
\hline & $\begin{array}{l}\text { affinis, } \\
\text { li, } 4\end{array}$ & $\begin{array}{l}\text { Smith, Melv. \& Standen, Manchester Memoirs, } \\
\text {.p. } 6 . \quad \text { N. }\end{array}$ \\
\hline
\end{tabular}


Bechuanaland. Ngami River (Passarge).

Transvaal. "Libonibo" (? Lebombo) (Wilms).

Matebililand. Meno's Kraal (Penther).

Rhodesia. Victoria Falls (Connolly).

Also known from North, West, and East Africa, and Northern Rhodesia.

von Martens (1897) places affinis, Smith, in the synonymy of ovum, while Melvill and Standen (1907) remark that affinis seems a distinct species.

References to both are given above for purpose of comparison.

var. ELATIOR, von Martens.

1866 Lanistes ovum, Ptrs., var. elatior, von Mts., Novit. Conch. ii. p. 291. pl. 70, f. 7, 8. D.F.

1879 Meladomus elatior, Bgt., Moll. de l'Égypte, p. 35.

Described from Niebohr River, $8^{\circ}$ N. lat. (Heuglin).

var. INGEns, Ancey.

1894 Meladomus ovum, Ptrs., var. ingens, Ancey, Mém. Soc. Zool. Fr. vii. p. 223 . $D$.

Described from Karonga, B.C.A.

\section{Lanistes purpureus (Jonas).}

1839 Ampullaria purpurea, Jonas, Archiv. f. Naturg. v, 1. p. 342. pl. 10, f. 1. D.F.

" Bulimus tristis, Jay, Catalogue of Shells, p. 121. pl. 7, f. 1. N.F.

1840 Meladomus bulimoides, Swainson, Treatise on Malac. p. 340. D.

1845 Lanistes purpurea, Jon. (=tristis, Jay), Trosch., Archiv. f. Naturg. xi, 1. p. 216. D.

1851, 2 Ampullaria purpurea, Jon.,Phil.,Conch.Cab.p.22.pl.6,f.1. D.F. 1854 Meladomus olivaceus, "Sow.," H. \& A. Ad., Gen. rec. Moll. i. p. 349 , and iii. pl. 37, f. $6 . F$.

1859 Lanistes purpureus, Jon., von Mts., Mal. Blätt. vi. p. 216. N. 1860 Ampullaria olivacea, "Sow." (=purpureus, Jonas), Morel., Séries Conch. ii. p. 108. N.

1866 Lanistes purpureus, Jon., von Mts., Novit. Conch. ii. p. 293. N. 1881

1897

" Smith, P.Z.S. p. 290. N.

" von Mts., D.-O.-Afr. p. 163. D.N.

Type of purpureus in Zool. Mus. Berlin; tristis in American Museum of Natural History, New York.

Hab. Lorenzo Marques. Tette (Peters).

Also found in East Africa and Zanzibar. 
Originally described as from the Swan River, Australia.

von Martens (1897) considers M. bloyeti and nitidissimus, Bgt., to be merely varieties of purpureus.

\section{FAMILY VIVIPARIDAE, Gray, 1847. \\ (P.Z.S. p. 155.) \\ (=Paludinidæ, Auctt.)}

Genus VIVIPARA, de Montfort, 1810.

(Conch. Syst. ii. p. 247, as Viviparus, emend. Dupuy, 1851, Hist. nat. des Moll. . . . qui vivent en France, p. 534.)

(=Vivipare, Lam., 1809, and Paludina, Lam., 1812.)

Type of Genus, V. fluviomm, de Montf. (vivipara, Lin.).

549. Vivipara capillata, von Frauenfeld.

1865 Vivipara capillata, Frnfd., P.Z.S. p. 659. D.

\begin{tabular}{|c|c|c|c|}
\hline , & $"$ & $"$ & $\begin{array}{l}\text { Verh. Zool. Ges. Wien, xv. p. } 533 . \\
\text { pl. 22. D.F. }\end{array}$ \\
\hline Paludina & ", & ", & Smith, P.Z.S. p. 717.pl. 74, f. 3, 4. \\
\hline Vivipara & , & , & $\begin{array}{l}\text { Kob., Conch. Cab. p. 174. pl. } 34, \text { f. } \\
\text { D.F. }\end{array}$ \\
\hline
\end{tabular}

Type in British Museum.

Hab. Zululand. Lake Sibayi (Toppin).

RhodesiA. ? Victoria Falls (Morrell, fide Preston; Dixey and Longstaff).

Lorenzo Marques. Rikatla (Junod).

Originally described from Lake Nyassa.

550. Vivipara passargei, von Martens.

1904 Vivipara passargei, von Mts., Die Kalahari, pp. 757;755, f. 3. D.F.

Type-ubi?

Hab. Bechuanaland. In marly sandstone at the Letter Tree, Botletle (subfossil, Passarge).

Founded on a single specimen, "similar to the East African V. unicolor."

551. Vivipara sambesiensis, Sturany.

1898 Vivipara unicolor, Oliv., var. sambesiensis, Stur., S.A. Moll. p. 85. pl. 3, f. 57-61. L.F.

1905

densestriata, Prest., Proc. Mal. Soc. vi.p. 300, f. 2. D.F.

1907 " sambesiensis, Stur., Kob., Conch. Cab. p. 172. pl. 33, f. $15,16 . \quad$ D.F. 
1907 Vivipara densestriata, Prest., Kob., Conch. Cab. p. 173. pl. 33, f. 17 . D.N.F.

1909 ", sambesiensis, Stur. (cum var. densestriata, Prest.), Kob., Abh. Senckenb. Naturf. Ges. Frankfurt, xxxii. p. 79.

Type of sambesiensis in Naturh. Hofmus. Vienna; densestriata in British Museum.

Hab. Rhodesia. Victoria Falls (sambesiensis, Penther; densestriata, Morrell).

I adopt Kobelt's synonymy, in the belief that only one variable species of Vivipara inhabits the Zambesi at the Victoria Falls. It seems probable that the reported occurrence of $V$. capillata, Frnfd., in the same locality also refers to sambesiensis.

\section{Genus CLEOPATRA, Troschel, 1857.}

(Gebiss der Schnecken, i. p. 100.)

Type of Genus, C. bulimoides (Oliv.).

522. Cleopatra ferruginea (Lea).

1850 Melania fermginea, Lea, P.Z.S. p. 182. D.

1851 " zanguebarensis, Petit, J. de C. ii.p. 263.pl. 7, f. 1. D.F. " $\quad$ " amana, Morel., J. de C. ii. p. 192. pl. 5, f. 9. D.F.

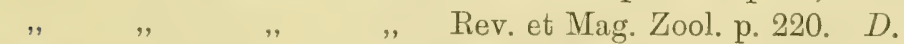

$1860 \quad$ " $\quad$ " $\quad$, Séries conch. ii. p. 117. D.

, $\quad$ " fermainea, Lea, Rve., Conch. Icon. pl. 21, f. 147. D.F. 1878 Puludomus africana, von Mts., Monats.-Ber. K. Akad. Wiss.

Berlin, p. 297. pl. 2, f. 11-13. D.F.

1879 Cleopatra kynganica and cameroni,Bgt., Moll.de l'Egypte,p.21. D. 1881 Paludomus ferrugineus, Lea, Smith, P.Z.S. p.294. pl.34, f.29. N.F. 1894 Cleopatra fermininea ", (=africana, von Mts.), Smith, Proc.

Mal. Soc. i. p. 167. N.
1897

, 1899

,

1911

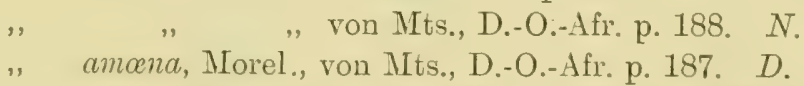

", ferruginea, Lea (=amana, Morel.), M.\& P., A.M.N.H. iv. p. 193. N.

Types of ferruginea and amena in British Museum.

Hab. Zululand. Manuan Creek (Anderson).

Lorenzo Marques. River: Quaqua, near the mouth of the Zambesi (Stuhlmann). Itschongove (fide Sturany). 
Cape of Good Hope. Prieska (Gibbons, fide M. \& P.).

This species, originally founded on a single specimen collected at Zanzibar, is rather widely distributed in East Africa, and has apparently been described under various different names. Melvill and Ponsonby, after comparison of the types, place $C$. amana in the synonymy, and von Martens cannot separate cameroni and kynganica, Bgt., from ferruginea; while Smith (1881) remarks that "Melania zangucbarica of Petit appears to be the same as this species; and P. africamus of Martens, if not identical, offers but slight distinctions."

553. Cleopatra morrelli, Preston.

1905 Cleopatra morrelli, Prest., Proc. Mal. Soc. vi. p. 300, f. 3. D.F. 1909 " morelli, "Smith," Kob., Conch. Cab. p. 391. pl. 76, f. 6, 7. D.F.

Type in British Museum.

Hab. Rhodesia. Victoria Falls (Morrell).

var. costata, Preston.

1905 Cleopatra morrelli, var. costata, Prest., Proc. Mal. Soc. vi. pp. 300, f. 4 ; 301. F.D.

Type in British Museum.

Hab. Rhodesia. Victoria Falls (Morrell).

FAMILY MELANIID AE, d’Orbigny, 1837.

(Moll. Canar. p. 77, as Melanidæ.)

Sub-FAmily MELANIIN无, H. \& A. Adams, 1854.

(Gen. rec. Moll. i. p. 294.)

Genus MELANIA, Lamarck, 1799.

(Mém. Soc. Hist. Nat. Paris, p. 75.)

Type of Genus, M. amarula (Lin.).

554. Melania amarula (Linné).

1758 Helix amamila, Lin., Syst. Nat., Ed. 10. i. p. $774 . \quad$ D.

$1764 \quad$ " $\quad$ Mus. Lud. Ulr. p. 672. D.

$1767 \quad$ " $\quad$ "Syst. Nat., Ed. 12. p. 1249. D.

1774 Buccinum amarula, Müll., Verm. ii. p. 137. D.

1786 Helix , Lin., Chem., Conch. Cab. ix, 2. p. 157. pl. 134, f. 1218, 19. D.F.

$1788 \quad$ " Gmel., Syst.Nat.,Ed.13.i,6.p.3656. D. 
Reference List of South African Non-marine NIollusca. 263

1824 Melania amarula, Lin., Sow., Genera of Shells, pl. 180, f. 1. F. $1828 \quad$ ", " Lam., Stark, Elem. Nat. Hist. ii. p. 53. D.

1838 , " , „ Desh., Hist. nat. An. s. Vert. viii. p. 431. D.

1839 ,, moreleti, Desh., Traité Elem. Conch. pl. 74, f. 13, 14. F'. 1854 Tiara amarula, Lin., H. \& A. Ad., Gen. 1'ec. Moll. i. p. 294, and iii. pl. 31, f. $3 . \quad F$.

1860 Melania ,, „, Rve., Conch. Icon. pl. 25, f. 177. D.F.

1877 ," , Brug., Brot, Conch. Cab. p. 289.pl.29, f.1. D.F'.

" " moreleti, Desh., Brot, ibid. p. 291. pl. 30, f. 2. D.F.

1880 ", (Tiara) amarula,Lin., von Mts., Meeresfauna Mauritius, p. 210 . N.

1884 Tiara amarula, Lin. (=moreleti, Desh.), Nev., Hand List, ii.

Type-ubi?

p. 278. N.

Hab. Natal. Izezela (Siveeney). Umkomans (Burnup).

Also known from Mauritius and Madagascar.

Nevill (1884) writes: "I think I may state positively that Deshayes' $M$. moreleti is the young of the typical form" (amarula), "notwithstanding the remarkable 'quasi" adult appearance that it invariably presents."

It is questionable whether the Natalian examples should be attributed to amavula or to the following species.

555. Melania coacta (Meuschen).

1780 Helix amarula, "Lin.," Born, Test. Mus. Caes. Vindobone, p. 391. pl. 16, f. 21 . D.F.

1787 Strombus coactus, Meuschen, Museum Geversianum, p. 294. D. 1877 Melania thiarella, Lam. (=coactus, Meusch.), Brot, Conch. Cab. p. 291. pl. 29, f. 3. D.F.

1897 „, coacta, Meusch., von Mts., D.-O.-Afr. p. 197. pl. 6, f. $36 . \quad D \cdot F$.

Type-ubi?

Hab. Lorenzo Marques, Rikatla; Nkomati River, near Morakouene (Junod).

von Martens mentions several localities in Zanzibar and East Africa.

556. Melania crawfordi, Brot.

1894 Melania craufordi, Brot, J. de C. xlii. p. 473. pl. 9, f. 5. D.F. Type in British Museum. 
Hab. Transvant. Middelburg (Crawford). Hennop's River and Crocodile River, Pretoria District (McBean; Connolly).

Cape of Good Hope. Sundays River, Port Elizabeth (Crawford).

557. Megania tuberculata (Müller).

1774 Nerita tuberculata, Müll., Verm. ii. p. 191. D.

1786

1842 Melania

1849

1859

1874 " tuberculata, Müll., Jick., Fauna N.-O.-Afr.p. 251.pl. 3, f. 7. pl. 7, f. 36 . D.T. F.

1875 ", inhambanica, von Mts., Brot, Conch. Cab. p. 66. pl. 7,

f. $6 . D \cdot F$.

1877 " tuberculata, Müll., Brot, ibid. p. 247. pl. 26, f. 11. D.F.

$1881 \quad$ " $\quad$, $\quad$ " Crosse, J. de C. xxix. p. 282. N.

$1884 \quad$,

1897,

1904 ,

1908

1909
" Chem., Conch. Cab. ix, 2. p. 189 pl. 136, f. 1261, 1262. D.F.

" Phil., Abb. u. Beschr. i. p. 4. pl. 1, 1. f. 14 . I). $I$.

Mouss., Moll. von Java, p. 73. pl. 11, f. $6,7$. D.F.

" Rve., Conch. Icon. pl. 13, f. 87. D.F. inhambanica, von Mts., Mal. Blätt. vi. p. 216. pl. 2,

f. 10. D.F.

Frankfurt, xxxii. p. 39. pl. 10, f. 5, 6. N.F.

Original in University Zool. Mus. Copenhagen.

Hab. Lorenzo Marques. Inhambane; Tette (Peters). Quaqua River (inhambanica, Stuhlmann).

Transvaal. Komati District (inhambanica, Schenck). Middelburg (Crawford).

Zululand East (Burnup).

Natal. Umgeni Lagoon (Burnup).

Bechuanaland. Makarrikarri Pan; Lake Ngami; Botletle District; Mori Mossetla, Ngami River (fossil, Passarge).

Described from Coromandel, and very widely distributed over Africa and parts of Asia, the coasts of the Mediterranean, Java, Borneo, and other islands of the Pacific Ocean. Jickeli (1874) includes in its synonymy: Strombus costatus and transversim striatus, Schröt.; Strombus vibex and $H$. acicula, Gmelin; $M$. fasciolata, Oliv.; ? Nerita lacustris, Brocchi; M. aspersa, Trosch.; porcata, 
Jonas; tamsii and flammigera, Dkr.; rivularis, Phil.; unifasciata and rothiana, Mouss.; indefinita, Lea; zengana and comersoni, Morel.; ?nevcombii, Lea ; judaica, Roth; tigrina, Hutt. ; pyramis, Bs.; exusta and vallacei, Rve.; punctulata, Grat.; abyssinica, Rüppell ; beryflina, Brot; layardi, Dohrn; and rubropunctata, Tristram.

Brot (1877) adds to the above M. suturalis, Phil. ; truncatula, Lam.; mauricia, Less. ; and incontaminata, muricata, and biscarce, Parreyss.

558. Melania victorie, Dohrn.

1865 Melania victoria, Dohrn, P.亡.S. p. 234. D.

$1877 \quad$ ", Brot, Conch. Cab. p.257.pl.26,f.2. D.F.

Originals in British Museum.

Hab. Rhodesia. Victoria Falls (Kirk; Morrell).

FAmily PALUDESTRINID AE, B. B. Woodward, 1903.

(Journ. of Conch. x. p. 355.)

(= Hydrobiidæ, Auctt.)

Genus PALUDESTRINA, d'Orbigny, 1840.

(Voy. Amer. Merid., Moll. p. 381.)

(=Hydrobia, Hartmann, 1821, nec Leach, in Coleoptera 1817.)

Type of Genus, P. auberiana, D'Orb.

559. Paludestrina alabastrina (Morelet).

1889 Hydrobia alabastrina, Morel., J. de C. xxxvii. p. 19. pl.2, f. 5. D.F.

Type in British Museum.

Hab. Cape of Good Hope. Port Elizabeth (Crawford).

560. Paludestrina tristis (Morelet).

1889 Hydrobia tristis, Morel., J. de C. xxxvii. p. 18. pl. 2, f. 4. D.F.

Type in British Museum.

Hab. Cape of Good Hope. Port Elizabeth (Crawford).

NataL. Lower Umkomaas; Winkel Spruit (Burnup).

561. Paludestrina zivellendamensis (Küster).

1852, 3 Paludina zwellendamensis, Krs., Küst., Conch. Cab. p. 53. Type-ubi?

pl. 10, f. 19, 20. D.F.

Hab. Cape of Good Hope. Zoetendals Valley, Swellendam District (Krauss).

Küster wrongly ascribes this species to Krauss' Südafr. Mollusken. The description in Conch. Cab. is from a shell in coll. Küster. 
FAMILY TRUNCATELLID AE, Gray, 1840.

(Syn. Brit. Mus. pp. 117, 148.)

Genus TRUNCATELLA, Risso, 1826.

(Hist. nat. Europe mérid. iv. p. 124.)

(=Truncatula, Leach, 1818 (ined.) ; Erpetrometra, Lowe, 1831; and Choristoma, de Cristofori and Jan, 1832.)

Type of Genus, T. lavigata, Risso.

562. Truncatella teres, Pfeiffer.

1856 Truncatella teres, Pfr., P.Z.S. p. 336. D.

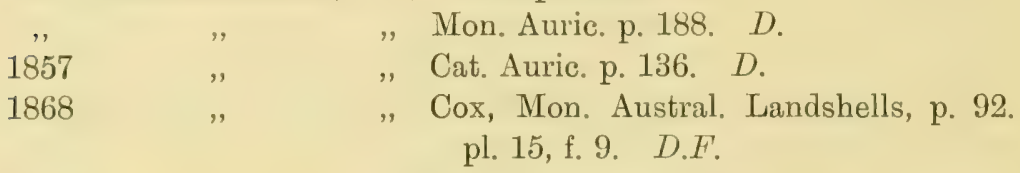

$1874 \quad$ ", , Jick., Fauna N.-O.-Afr. p. 188. D.

Type in British Museum.

Hab. Cape of Good Hope. Port Elizabeth (Crawford; Penther; Farquhar).

First recorded from Mauritius and Australia; also known from the Red Sea.

Genus TOMICHIA, Benson, 1851.

(A.M.N.H. vii. p. 378.)

Type of Genus, T. ventricosa (Rve.).

563. Tomichia ventricosa (Reeve).

1842 Truncatella ventricosa, Sow., Rve., Conch. Syst. ii.p. 94. pl. 182, f. 2. $F$.

1846

1848

, ,

," Pfr., Zeitschr.f. Malak.iii.p.189. D. , (=capensis, Krs. in litt.), Krs., Südafr. Moll.p.87.pl.5,f.22, D.F.

1851 Tomichia , Bs., A.M.N.H. vii. p. $379 . \quad D$.

1855 Truncatella

Küst., Conch. Cab. p. 13. pl. 2, f. $27-31 . \quad D \cdot F$.

"Tomichia „, „, H. \& A. Ad., Gen. rec. Moll. ii. p. 313 , and iii. pl. 88, f. 4. D.F.

1885 Hydrobia caledonensis, Chaper, Bull. Soc. Zool. Fr. x. p. 484. pl. 11, f. 6. D.F.

1889 Morel., J. de C.xxxvii.p. 18. $N$. 
1901 Tomichia ventricosa, Sow. (=caledonensis, Chaper), Ancey, J. de C. xlix. p. 225. N.

Type of ventricosa in British Museum; caledonensis in École des Mines, Paris.

Hab. Cape of Good Hope. Cape Flats; Zoetendals Valley (Krauss). “Baszaarm's" (Baas Harman's) Kraal; Michelville, or Holloway's Halfway House (Benson). Port Elizabeth (Crawford; Farquhar). Coega (Crawford). Near Caledon (caledonensis, Chaper). Milnerton (Connolly). Vogel Vlei, Calvinia Division (fossil, Moffatt).

var. BREvis, Krauss.

1848 Truncatella ventricosa, Sow.,var.brevis, Krs., Südafr.Moll.p.87. D. Type in Stuttgart Museum.

Hab. CAPE of Good Hope. Cape Flats; Zoetendals Valley (Krauss). Eerste River (Connolly). Lorens River, Somerset District (Holub).

FAmiry ASSEMANIID A, H. \& A. Adams, 1856.

(Gen. rec. Moll. ii. p. 314, as Assiminiidæ, emend. B. B. Woodward, 1903, Journ. of Conch. x. p. 356.)

Genus ASSEMANIA, Leach in Fleming, 1828.

(Hist. Brit. Anim. p. 275, as Assiminea, emend. Knight, 1900, Journ. of Conch. ix. p. 275, and B. B. Woodward, 1903, Journ. of Conch. x. pp. 356, 366.) (=Assiminia, Assaminea, Auctt.)

Type of Genus, A. grayana, Leach.

564. Assemania bifasciata, Nevill.

1880 Assiminia bifasciata, Nev., Journ.As.Soc. Bengal,xlix,2.p.162. D. 1887 Assiminea ", , Bttg., Jahrb. d. Deutsch. Mal. Ges.

Type in Indian Museum, Calcutta. xiv. p. 161 . $D$.

Hab. Natal. Port Natal (Nevill). Umhlatusani; Umlaas and Umkomaas Rivers (Burnup).

565. Assemania fasciata (Krauss).

1848 Paludina fasciata, Krs., Südafr. Moll. p. 86. pl. 5, f. 18. D.F. 1887 Assiminea ", , Bttg., Jahrb. d. Deutsch. Mal. Ges.

Type in Stuttgart Museum. xiv. p. 174. $D$.

Hab. Cape of Good Hope. Knysna and Zoetendals Valleys (Krauss). 


\section{Assemania hidalgor (Gassies).}

1869 Hydrocena hidalgoi, Gass., J. de C. xvii. p. 78. D.

1882 Assiminea granum, Morel., J.de C.xxx.pp.105, 198.pl.4,f.8. D.N.F. 1883 ,. " , " (hidalgoi, Gass.), Morel., J. de C. xxxi.

1887 „, hidalgoi, Gass., Bttg., Jahrb. d. Deutsch. Mal. Ges. xiv. p. 180.1 ).

Both types in British Museum.

Hab. Natal. Amahlongwana Lagoon (Burnup).

Hidalgoi was described from New Caledonia; granum from Mauritius. The actual types differ considerably, but in Morelet's original type set of granum is one shell which is quite inseparable from hidalgoi. Under these circumstances I do not care to gainsay Morelet's expressed opinion as to the synonymy of his own species.

The Natal representatives are typical hidalgoi.

\section{Assemania inysnaensis (Krauss).}

1848 Paludina knysnaensis, Krs., Südafr. Moll. p. 86. pl.5, f. 17. D.F. 1887 Assiminea , , Bttg., Jahrb. d. Deutsch. Mal. Ges.

Type in Stuttgart Museum. xiv. p. 182. $D$.

Hab. Cape of Good Hope. Knysna and Zoetendals Valleys (Krauss). Marshy ground near the beach, Port Elizabeth (Crawford).

\section{Assemania ovata (Krauss).}

1848 Paludina ovata, Krs., Südafi. Moll. p. 85. pl. 5, f. 16. D.F. 1863 ", " , Frnfd., Verh. Wien Zool. Bot. Ges. xiii. p. 211. $N$.

1887 Assimined ,, , Bttg., Jahrb. d. Deutsch. Mal. Ges. xiv. p. 194. $D$.

Type in Stuttgart Museum.

Hab. Cape of Good Hope. Knysna and Zoetendals Valleys (Krauss). Zwartkops River Marshes, Port Elizabeth (Crawford).

569. Assemania ponsonbyi, Morelet.

Assiminea ponsonbyi, Böttger in Litt.

$1889 \quad$ ", Morel., J. de C. xxxvii. p. 17.pl.2,f.6. D.F.

Type in British Museum.

Hab. Cape of Good Hore. Rufane Vale, Port Elizabeth (Crawford). 
570. Assemania sinica, Nevill.

1880 Assiminia sinensis, Nev.,Journ.Asiat.Soc.Bengal,xlix,2.p.161. D. 1887 Assiminea sinica, Nev. (for sinensis, Nev., not chinensis, Pfr.), Bttg., Jahrb. d. Deutsch. Mal. Ges. xiv. p. 203. D.

Type in Indian Museum, Calcutta.

Hab. Natal. Wydenham; Umkomaas (Burnup).

Described from Hong Kong.

571. Assemania umlaasiana, Smith.

1902 Assiminia umlaasiana, Smith, Journ. of Conch. x. p. 248. pl. 4, f. 3. D.F.

Type in British Museum.

Hab. Natal. In an ocean cave at Isipingo Rocks, half a mile south of Umlaas River mouth (Burnup).

Order ASPIDOBRANCHIA, Schweigger, 1820.

(Naturges. d. Skeletlose Thiere, p. 720, as Aspidobranchiata ; emend. Menke, 1830.)

Sub-Order RHIPIDOGLosSA, Troschel, 1847.

(Archiv. f. Naturg. xiii, 2. p. 385, and Wiegmann's Handbuch der Zool., Ed. 3. 1848.)

FAMiLY HYDROCENIDA:, Bourguignat, 1877.

(Descr. de deux nouveaux Genxes Algériens, p. 38.)

Genus HYDROCENA, Parreyss, 1846.

(Hermannsen's Indicis Generum Malac. i. p. 546.)

Type of Genus, H. cattaroensis (Pfr.).

572. Hydrocena noticola, Benson.

1856 Hydrocena noticola, Bs., A.M.N.H. xviii. p. 439. D.

1858

„, Pfr., Mon. Pneum. ii. p. 158. D.

1897 Assiminea tyttha, M. \& P., A.M.N.H. xix. p.639. pl.17, f.11. D.F.

Type of tyttha in British Museum; noticola-ubi?

Hab. Cape of Good Hope. Table Mountain, Camps Bay (Layard). Simonstown; Hout Bay (Connolly). Grahamstown; Kowie; Bathurst; Port Elizabeth (Farquhar).

Natal. Howick (tyttha); Scottburgh; Karkloof; Dargle; Game Pass ; Ntimbankulu (Burnup).

The type set of tyttha has been most carefully compared with a large series of noticola from Table Mountain, with the result that no specific difference has been found between them. 


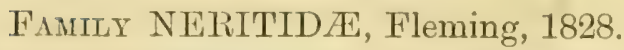

(Hist. Brit. Anim. p. 318, as Neritadx, emend. Turton, 1831, Manual, pp. 10, 138.)

Genus THEODOXIS, de Montfort, 1810.

(Conch. Syst. ii. p. 350.)

(=Neritina, Lam., 1822 [Neritine, 1809].)

Type of Genus, T. lutetianus, de Montf. (fluviatitis, Lin.).

573. Theodoxis kNorri (Récluz).

1841 Neritina knorri, Récl., Rev. Zool. Soc. Cuv. p. 274. D.

1849 " knorrii, Récl., Sow., Thesaurus Conch. ii. p. 511. pl. 111 , f. 78 . pl. 113 , f. 150 . D.F.

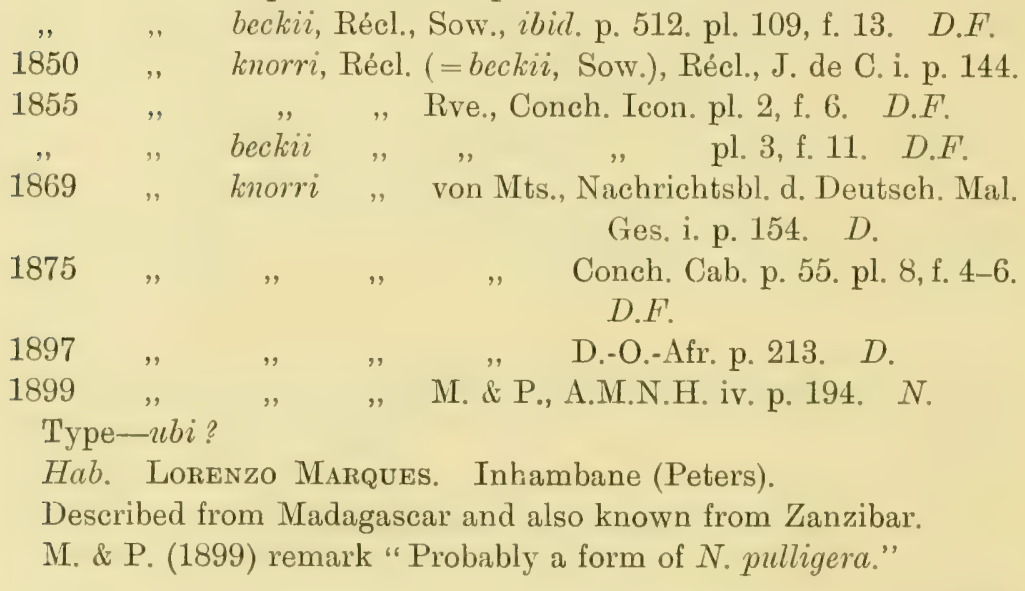

574. Theodoxis natalensis (Reeve).

[S.A.M.]

1848 Neritina zebra, "Lam.," Krs., Südafr. Moll. p. 88. N.

1855 ", natalensis, Rve., Conch. Icon. pl. 16, f. 75. D.F.

1877 " " , von Mts., Conch. Cab. p. 96. pl. 11, f. $10,11,15$. D.F.

1897

D.-O.-Afr. p. $213 . \quad D$.

Originals in British Museum, type not specified.

Hab. Natal. Umgeni River (Krauss). Umkomaas (Burnup).

Lorenzo Marques. Inhambane; Tette (Peters). Delagoa (Junod).

Also found in Central East Africa.

575. Theodoxis souverbianus (Montrouzier).

1861 Neritina souverbiana, Montr., Gassies, Act. Soc. Linn. Bordeaux, xxiv. p. 309. pl, 8, f. 7. D.F. 
Reference List of South African Non-marine Mollusca. 271

1863 Neritina souverbiana, Montr., J.de C. xi.pp. 75, 175. pl. 5, f. 5. D.F. 1871 " pulcherrima, Angas, P.Z.S. pp. 19, 96. pl.1, f. 25. D.F. 1879 " souverbiana, Montr. (=pulcherrima, Ang.), von Mts., Conch. Cab. p. 251.pl. 23, f. 29-31. D.F. $1881 \quad$ " $\quad$ " Montr., Morel., J. de C. xxix. p. 208. D.

$1883 \quad " \quad$ " $\quad$ " $\quad$ " $\quad$ xxxi. p. 204. N. $1910 \quad$ " $\quad$ " $\quad$, Smith,Ann.Natal Mus.ii.p.204. N.

Type of pulcherrima in British Museum.

Hab. Natal. Congella (Burnup).

Souverbianus was originally described from New Caledonia, and pulcherrima from Port Jackson.

Class LAMELLIBRANCHIA, de Blainville, 1816.

(Bull. Soc. Philom. p. 122, as Lamellibranches.)

Order EULAMEllibranchia, Pelseneer, 1892.

(Bull. Sci. Fr. et Belg. p. 365.)

SUb-Order SUBMYTILACEA, de Blainville, 1825.

(Man, de Malac. p. 537.)

FAMILY UNIONID无, Fleming, 1828.

(Hist. British Animals, p. 415.)

Dr. F. Haas, of Frankfurt-am-Main, has very kindly furnished me with much valuable information regarding this family, which he has collected for his own forthcoming monograph on the subject; an act of exceptional courtesy, for which I am greatly indebted to him.

Genus CAFFERIA, Simpson, 1900.

(Proc. U.S. Nat. Mus. xxii. p. 824.)

Type of Genus, C. caffra (Krs.).

576. CAFFERIA CAFFra (Krauss).

[S.A.M.]

1848 Unio caffer, Krs., Südafr. Moll. p. 18. pl. 1, f. 14. D.F.

" $\quad$ zeyheri, Mke., Zeitschr. f. Malak. v. p. 28. D.

1851 " cyamus, Phil., ", v. p. 125. D.

1856 ", verrauxi, Charpentier, Küst., Conch. Cab. p. 150. pl. 43 (1855), f. 6. D.F.

" $\quad$ " verreauxianus, Lea, Proc. Acad. Nat. Sci. Phila. viii. p.94. D.

" $\quad$ " africanus, Lea, ibid. p. $94 . \quad$ D.

", " caffer, Krs., Küst., Conch. Cab.p.143.pl.42(1855),f.2,3. D.F. 
1857 Unio verreanxianus, Lea, Obs. G. Unio, vi.p. 21.pl.27, f. 16. D.F.

" " africanus, Lea, ibid., p. 20. pl. 27, f. 15. D.F.

" " " " Rve., Conch. Icon. pl. 22, f. 100. D.F.

1858 " , " , Journ. Acad. Nat. Sci. Phila. iii. p. 300. pl. 27, f. 15. D.F.

" " verreanxianus, Lea, ibid. p. 301. pl. 27, f. 16. D.F.

1859 " navigioliformis " Proc. Acad. Nat. Sci. Phila. viii. p. $152 . D$.

" , , $\quad$, Obs. G. Unio, vii. p. 63. pl. 37, f. 124. D.F.

$1860 \quad$, , , Journ. Acad. Nat. Sci. Phila. iv. p. 245. pl. 37, f. 124. D.F.

1864 " natalensis ", Proc. Acad. Nat.Sci.Phila.p. 113. D. , Obs. G. Unio, xi. p. 63. pl. 20, f. 57. D.F.

1865 ", navigoliformis ," Rve., Conch. Icon. pl.24, f. 114. D.F.

1866 " caffer, Krs., Rve., Conch. Icon. pl. 41, f. 226. D.F.

, natalensis, Lea, Journ. Acad. Nat. Sci. Phila. vi. p. 59. pl. 20 , f. 57. D.F.

$1868 \quad$ " $\quad$ " $\quad$, Sow., Conch. Icon. pl. 71, f. $362 . \quad$ D.F.

" "verreauianus, " " $\quad$ pl. 69, f 352. D.F.

", ", rectilinearis, Sow., Conch. Icon. pl. 65, f. 332 . D.F.

1880 ", caffer, Krs., Crvn., P.Z.S. p.618. N.

1885 ", vaalensis, Chaper, Bull. Soc. Zool. Fr. x. p. 480. pl. 11, f. 1-3. D.F.

1889 ", natalensis, Lea, and verreauxi, Charp., von Mts., Sitz.-

Ber. Ges. Nat. Fr. Berlin, p. 165. L.

1891 „ caffer, Krs., Smith, A.M.N.H. viii.p. 317. D. and Synonymy.

", " verreauxi, Charp., Smith, ibid. p. 319. N.

1898 " caffer, Krs., var. pentheri, Stur., S.A. Moll. p. 91. pl. 3, f. 64,65 . D.F.

1900 Nodularia (Cafferia) caffer, Krs., Simpson, Proc. U.S. Nat. Mus. xxii. p. 825. Synonymy.

Type of caffra in Stuttgart Museum ; vaalensis in École des Mines, Paris; zeyheri lost; africanus, natalensis, and verreauxianus in U.S. National Museum, Washington; navigioliformis and rectilinearis in British Museum; var. pentheri in Naturh. Hofmus. Vienna.

Hab. "South AFrica" (cyamus, in coll. Largilliert).

Natal (Vahlberg). Umsinduzi River, Pietermaritzburg (Burnup). Umpingave River (natalensis, McKen).

Transvadu. Common round Pretoria and in Crocodile River (Connolly). Vaal River, Standerton (Miss Livingston). Between 
Kimberley and Christiania (natalensis, Schenck). Kalkspruit, between Vaal and Heidelberg (verreauxi, Schenck, fide von Martens).

Orange Free State. Riet River, Winburg (fide Sturany). Rhenoster River, south of Heilbron (Connolly).

CaPe of Good Hope (africanus and verreauxianus, Verreaux; zeyheri, Zeyher). Izeli River, near King Williamstown (Godfrey). Port Elizabeth District (Crawford). Retreat (Lightfoot). Vaal River, Barclay (vaalensis, Chaper). "Soutenthal" (Zoetendals) Valley (verreauxi, Verreaux).

Little Namaland. Orange River, Henkries (Lightfoot).

Griqualand West. Imvani and Riverton, Vaal River (Miss Wilman).

British Bechuanaland. Hartz River, Taungs (Miss Wilman).

Rhodesia. Panda ma tinka, R. Zambesi (var. pentheri, Penther).

Smith (1891) places africanus, natalensis, verreauxianus, and vaalensis in the synonymy of caffra; Simpson (1900), while further admitting navigioliformis and rectilinearis, considers africanus and vaalensis worthy of varietal rank. Dr. Haas writes that verreauxianus, navigioliformis, natalensis, rectilinearis, africanus, vaalensis, and pentheri are inseparable from caffra, and that zeyheri and cyamus must also belong to this species.

577. Cafferia mashone (Preston).

1910 Unio mashonce, Prest., A.M.N.H. vi. p. 61. pl. 4, f. 10. D.F.

Type in British Museum.

Hab. RHodesia. A sluit about 16 miles from Enkeldoorn, Mashonaland (Miss Sharpe-Young).

Very similar to caffra, from which it may be separable through the absence of the umbonal scar.

578. Cafferia mossambicensis (Peters).

1859 Unio mossambicensis, Ptrs., von Mts., Mal. Blätt. vi. p. 218. pl. 3, f. 3-5. D.F.

1885 , Charmes, Bull. Soc. Mal. Fr. ii. p. $166 . \quad \mathrm{N}$.

1897 von Mts., D.-O.-Afr. p. 225. pl. 7, f. 2. D.F.

Type in Zool. Mus. Berlin.

Hab. Lorenzo Marques. Tette (Peters).

Also reported from Central Africa. 
Genus UNIO, Retz, 1788.

(Dissert. Nov. Test. Genern, Lund. p. 16.)

Type of Genus, U. pictorum (Lin.).

The four species which follow are left in Unio, to which they were originally attributed, pending further investigation. I have Dr. Haas' authority for stating that they do not really belong to that Genus, but that it is doubtful whether either of them can be placed in Cafferia.

579. Unio Fissidens, Böttger.

1886 Unio (Hyridella) fissidens, Bttg., Ber. Senckenb. Naturf. Ges. Frankfurt, p. 27. pl. 2, f.6,7.D.F. Bttg., Abh. Senckenb. Naturf. Ges. Frankfurt, xxxii. p. 453. N.

Type in Senckenburg Mus. Frankfurt.

Hab. British Bechuanaland. Nosob-Hygap watercourse, east of Kebeum (subfossil, Nolte). Witkop (subfossil, Schultze; Rogers).

580. Unio hygapanus, Böttger.

1886 Unio (Hyridella) hygapanus, Bttg., Ber. Senckenb. Naturf. Ges. Frankfurt, p. 26. pl. 2, f. 5. D.F.

Type in Senckenberg Mus. Frankfurt.

Hab. British Bechuanaland. Nosob-Hygap watercourse, east of Kebeum (subfossil, Nolte).

Described from a single left valve; possibly only a less highly sculptured form of fissidens.

581. Unio Kunenensis, Mousson.

1887 Unio kunenensis, Mouss., J. de C.xxxv. p. 300. pl. 12, f. 10. D.F. 1891 " $\quad$ "Smith, A.M.N.H. viii. p. 319. N. $1904 \quad$ ", $\quad$ von Mts., Die Kalahari, p. 758. N.

Type in Zurich Museum.

Hab. Ovampoland. Kunene River (Schinz).

Bechuanaland. Okavango and Botletle District, and in the salt pans of the Makarrikarri brook (fossil, Passarge).

"Nearly allied to $U$. hauttecoeuri, Bgt., and other species from the East African Lakes" (von Martens).

582. Unio zambesiensis, Preston.

1905 Unio zambesiensis, Prest., Proc. Mal. Soc.vi.pp.300,f.1;301. D.F. Type in British Museum.

Hab. Rhodesia. Victoria Falls (Morrell). 
FAMIL MUTELID $\mathrm{A}$, Gray, 1847.

(P.Z.S. p. 197, as Muteladœ, emend. H. \& A. Adams, 1857, Gen. rec. Moll, ii. p. 505.)

Genus SPATHA, Lea, 1838.

(Trans. Amer. Phil. Soc. vi. p. 141.)

Type of Genus, S. mbens, Lea.

583. Spatha mattenguensis, Sturany.

1898 Spatha maitenguensis, Stur., S.A. Moll. p. 92. pl. 3, f.66. D.F.

Type in Naturh. Hofmus. Vienna.

Hab. Rhodesra. Maitengue River (Penther).

Described from a single example, very nearly allied to wahlbergi (Kis.s.).

584. Spatha petersi, von Martens.

1859 Spatha petersi, von Mts., Mal. Blätt. vi. p. 218. pl. 3, f. 1, 2. D.F. 1864 " modesta, Lea, Proc. Acad. Nat. Sci. Phila. p. 109. D.

" $\quad, \quad$, $\quad$ Obs. G. Unio, xi.p. 41.pl.13, f. 35. D.F. 1889 „, petersi, von Mts., Sitz.-Ber. Ges. Nat. Fr.Berlin,p. 164. N. $1897 \quad$ " , " (=modesta, Lea), von Mts., D.-O.-Afr. p. 251. $D$.

Type of petersi in Zool. Mus. Berlin; modesta in U.S. National Mus. Washington.

Hab. Lorenzo Marques. Tette (Peters). Itschongove (Schenck). Rikatla; Nkomati River (Junod).

Also found in Central Africa. S. modesta was described from Mozambique.

\section{Spatha Wahlbergi (Krauss).}

1848 Iridina wahlbergi, Krs., Südafr. Moll. p. 19. pl. 2, f. 1. D.F. 1859 Spatha ", " von Mts., Mal. Blätt. vi. p. 217. N. 1864 ", natalensis, Lea, Proc. Acad. Nat. Sci. Phila. p. 113. D. $1866 \quad$ ", , , Journ. Acad. Nat. Sci. Phila. vi. p. 64. pl. 20, f. 58. D.F.

, " hartmanni, von Mts., Mal. Blätt. xiii. p. 10. D.

1867 " natalensis, Lea, Obs. G. Unio, xi. p. 68. pl. 20, f. 58. D.F. Anodon tabula, Sow., Conch. Icon. pl. 18, f. 68. D.F.

1874 Spatha hartmanni, von Mts., Jick., Fauna N.-O.-Afr.p. 263. pl. 8, f. 2. D.F. 
1875 Spatha natalensis, Lea, Cless., ibid. p. 189. pl. 62, f. 7, 8. D.F.

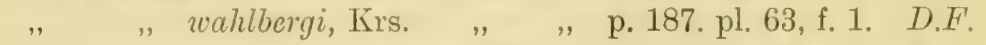

$1891 \quad$ " " $\quad$ " von Mts., Sitz.-Ber. Ges. Nat. Fr. Berlin, p. $17 . \mathrm{N}$.

\begin{tabular}{|c|c|c|c|}
\hline Mutela & & & Smith, A.M.N.H. viii. p. $319 . \quad$ N. \\
\hline , & & & $\begin{array}{l}\text { Simpson, Proc. U.S. Nat. Mus. xx } \\
\text { p. } 898 . \text { Synonymy. }\end{array}$ \\
\hline
\end{tabular}

1909 ". " , Kob., Abh. Senckenb. Naturf. Ges. Frankfurt, xxxii. p. 50. N.

Type of wahlbergi in Stockholm Museum; natalensis in U.S. National Mus. Washington.

Hab. Transvadu. Aapies River (IVahlberg; Farquhar). Crocodile River (Day). Middelburg (Crawford).

Lorenzo Marques. Tette (Peters).

Zululand. Enseleni River (Anderson).

Natal. Umpingave River (natalensis, MeKen).

Rhodesia. Bulawayo (in coll. Crawford).

Also found in East Africa.

von Martens (1897) remarks that S. natalensis, Lea, appears to differ little from bloyeti, Bgt.; Sturany and Simpson, however, place it in the synonymy of wahlbergi.

Kobelt (1909) states that he is unable to separate spathuliformis, Bgt., from wahlbergi, and includes Spathella bourguignati, Ancey, as a variety of Krauss' species.

val. DoRsalis, von Martens.

1859 Spatha wahlbergi, "Krs.," von Mts., Mal. Blätt. vi. p. 217.

1897 " Krs., var. dorsalis, von Mts., D.-O.-Afr. p. 247. $D$.

Hab. Lorenzo Marques. Tette; Sena (Peters).

Fanily CYRENIDE, Gray, 1840.

(Syn. Brit. Mus. pp. 133, 149.)

Genus CORBICULA, von Mühlfeld, 1811.

(Mag. Ges. Naturf. Fr. Berlin, p. 56.)

Type of Genus, C. fluminalis (Müll.).

It would require intimate acquaintance with the types, and long sets of each variety, to unravel the extraordinary tangle into which the African species of Corbicula have been woven. 
C. Anminalis (Müll.) was described from the River Euphrates in 1774, and consobrina, Calilaud (Voy. à Meroe, ii. pl. 61, f. 10, 11), from Egypt in 1823.

C. radiata, Parr, and pusilla, Parr. (Abb. u. Beschr. ii. p. 78. pl. 11, 4. f. 7), from Egypt, owe their origin to Philippi, 1846.

In 1848, however, Krauss propounded the name africana, comprising two South African varieties, olivacea and albida, with which he identified radiata and pusilla respectively. Olivacea is the type form of africana : albida was raised by Clessin (1877) to the rank of a species, and will probably prove to be identical with, and have precedence of, $C$. oliphantensis, Crvn. Clessin also described and figured a $C$.natalensis, Krs., of which no notice has been taken by subsequent authors.

Jickeli (1874) dealt with radiatc and pusilla as distinct species, but had not sufficient material to express an opinion as to the correctness of Krauss' views on the subject.

von Martens (1897) united pusilla with radiata, but separated the latter from africana, var. olivacea; Böttger, however (1910), considered radiata and africana identical.

Pallary (Mém. Instit. Égypt. vi, 1. 1909, pp. 71, 72) appears to prove that radiata and pusilla are both immature forms of consobrina, which last was placed by Jickeli (1874) and Westerlund (1890) in the synonymy of fluminalis, Müll.

The localities given in the following pages are, for the most part, correct for the varieties to which they refer, but the true degree of inter-relationship of the latter is obviously far too large a subject to admit of adequate discussion within the limits of the present work.

586. CoRbiCULA AFRICANA (Krauss).

1848 Cyrena africana, var. olivacea, Krs. (=gauritziana, Krs. in litt. and radiata, Parr.), Krs., Südafr. Moll.p. 8.pl.1, f. 8. D.F. 1854 Corbicula africana, Krs., Desh., Cat. Conchif. p. $222 . \quad$ D. 1859 Cyrena $\quad$ " , von Mts., Mal. Blätt. vi. p. 220. N. 1866 Corbicula ", " Prime, Ann. Lyc. N.H. New York, viii. p. 224, f. 57. D.F.

1877 , " , " Cless., Conch. Cab. p. 156. pl. 27, f. 22-24. D.F.

$1904 \quad$ " $\quad$ " $\quad$ von Mts., Die Kalahari, p. 758. N.

Type in Stuttgart Museum.

Hab. Cape of Good Hope. Gauritz River (Krauss). Swellendam (Layard). Gamtoos R. (Farquhar). Port Elizabeth (Crawford). 
Griqualand West. Riverton, Vaal River (very large specimens, $19 \times 17 \mathrm{~mm}$, Miss Wilman).

Little Namaland. Orange River, Henkries (Lightfoot).

British Bechuanaland. Hartz River, Taungs (Miss Wilman).

Bechuanaland. Letter Tree, Botletle River (Passarge).

Lorenzo Mlarques. Itschongove (Schenck). Tette (Peters).

Zululand East (Toppin; Burnup).

Natal. Nonoti River (Miss Brown).

Orange Free State. Kroonstad (Miss Hickey).

Transvaal. Vaal River, Standerton (Miss Livingston). Lepenula River (Wahlberg).

\section{Corbicula albida (Krauss).}

1848 Cyrence africana, var. albida, Krs. (=pusilla, Parr.), Krs., Südafr. Moll. pp. 8, 9. D.

1877 Corbicula albida, Krs., Cless., Conch. Cab. p. 156. pl. 27, f. 25,26 . L. $t$.

Type in Stockholm Museum.

Hab. TRansvaal. River Lepenula (Wahlberg).

588. Corbicula astartina (von Martens).

1859 Cyrena astartina, von Mts., Mal.Blätt.vi. p. 219. pl.3, f.6, 7. D.F. 1897 Corbicula ," , , D.-O.-Afr. p. 260. D.

Type in Zool. Mus. Berlin.

Hab. Lonenzo Marques. Tette (Peters).

Also recorded from Nyassa and the Schire River, East Africa, and Mterize River, Northern Rhodesia.

\section{Corbicula fluminalis (Müller).}

1774 Tellina fluminalis, Müll., Verm. ii. p. 205. D.

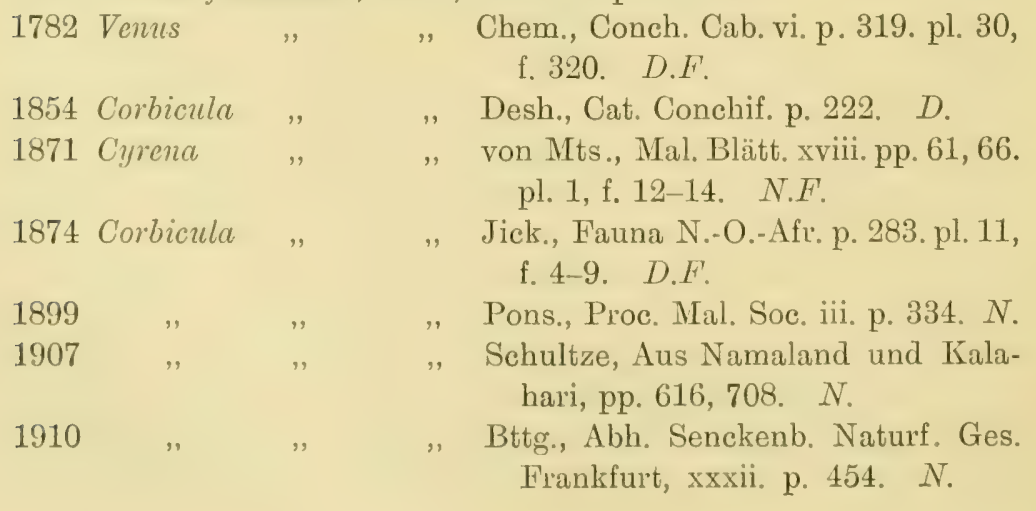


Type-ubi? Rogers).

Hab. British Bechuanaland. IVitkop (subfossil, Schultze;

Chronicled, living, from North-East, West, and Central, but not from East or South Africa.

The following species have, rightly or wrongly, been included by various authors in the synonymy of fluminatis :-

euphratica, cor, orientalis, and fuscata, Lam.; consobrina, Caill. ; saulcyi, Bgt.; and frivaldskyana, Zelebor.

\section{Corbicula natalensis, Krauss.}

1877 Corbicula natalensis, Krs. in litt., Cless., Conch. Cab. p. 155. pl. 27, f. 19-21. D.F.

Type in Stutgart Museum.

Hab. Natal (Queinzius).

591. Corbicula oliphantensis, Craven.

1880 Corbicula oliphantensis, Crvn., P.Z.S. p. 618. pl. 57, f. 12 . D.F.

Type in British Museum.

Hab. Transvaal. Oliphants River (Craven).

CAPE of Good Hope. Gamtoos River (Crawford).

It seems hardly possible that this species will eventually prove to be separable from albida, Krs.

592. Corbicula radiata (Parreyss).

1846 Cyrena radiata, Parr., Phil., Abb. u. Beschr. ii. p. 78. pl. 11, 4,

1848

1854 Corbicula

1866 Cyrena ,"

1874 Corbicula

1877

1878

1881

1892

1897

1904

1906

\section{f. $8 . \quad D . F$}

, Krs., Südafr. Moll. p. 8. N.

Phil., Desh., Cat. Conchif. p. 222. D.

", von Mts., Mal. Blätt. xiii. p. 14. $N$.

" Jick., Fauna N.-O.دAfr. p. 287. pl. 11, f. $10 . D . F$.

Parr., Smith, P.Z.S. p. 718. N.

" Cless., Conch. Cab. p. 162. pl. 28 (1877), f. 16-18. D.F.

" Smith, P.Z.S. p. 295. N.

" $\quad$ A.M.N.H. x. p. 126. N.

Phil., von Mts., D.-O.-Afr.p. 259. D.

Parr., Smith, Proc. Mal. Soc. vi. p. 100. N.

", Germain, Bull. Mus. Hist. Nat. Paris, p. $307 . N$. 
1910 Corbicula radiata, Phil., Bttg., Abh. Senckenb. Naturf. Ges. Frankfurt, xxxii. p. 454. $N$.

Type- ubi?

Hab. Transvaal. Potchefstroom (Miss Livingston).

British Bechuanaland. Witkop (subfossil, Schultze).

Lorenzo Marques. River Zambesi (Brancksik).

Described from Egypt, and recorded from East and Central Africa and Northern Rhodesia. Smith (1904) includes C. tanganikana, Bgt., in the synonymy of radiata, to which Germain (1906) adds C. nyassana, Bgt.

\section{Genus SPHARRIUM, Scopoli, 1777. \\ (Introd. ad. Hist. nat. p. 397.) \\ (=Cyclas, Brug., 1792.) \\ Type of Genus, S. comeum (Lin.). \\ 593. Sphertum Capense (Krauss).}

1848 Cyclas capensis, Krs., Südafr. Moll. p. 7. pl. 1, f. 6. D.F.

1854 Spherium capense, Krs., Desh., Cat. Conchif. p. 265. D.

1874 Cyclas capensis, Krs.,Jick., Fauna N.-O.-Afr.p.291.pl.11,f.14. D.F. 1877 Spherin capense, Krs., Cless., Conch. Cab. p.93.pl.10,f.3-5. D.F. 1883 ", subcapense, Bgt. (=capensis, Jick.), Bgt., Ann. Sci. nat. Paris, xv. p. 133. N.

1908 " capense, Krs. (= subcapense, Bgt.), Neuville \& Anthony, Ann. Sci. nat. Paris, viii. p. 338. N.

Type of capense in Stuttgart Museum; subcapense in Geneva Museum.

Hab. Cape of Good Hope. Knysna River (Krauss).

Rhodesia. Makabusi Falls, near Salisbury (Miss Weineck).

Without knowledge of the types it is impossible to say whether Bourguignat (1883) was right in separating Jickeli's Abyssinian form from capense, Krs., but, judging from the respective figures, there appears to be good ground for his decision.

\section{Spherium ferrugineum (Krauss).}

1848 Cyclas fermginea, Krs., Südafr. Moll. p. 7. pl. 1, f. 7. D.F. 1854 Pisum parasiticum, Parreyss, Desh., Cat. Conchif. p. 280. D. , , fervugineum, Krs., Desh., Cat. Conchif. p. 281. D.

1874 Limosina ferruginea, Krs., Jick., Fauna N.-O.-Afr. p. 293.pl. 11, f. 16,17 . D.F. f. 1-4. D.F. 
1883 Enpera parasitica, Parr., Bgt., Ann. Sci.nat. Paris, xv. p. 133. N. " $\quad$ " jickelii, Bgt., ivid. p. 134. D.

1892 Limosina parasitica, Parr., Smith, A.M.N.H. x. p. 126. N. 1897 Eupera $\quad, \quad$ Desh., von Mts., D.-O.-Afr. p. 261. N. 1909 ", " Parr., Pallary, Mém. Inst. Égypt. vi, 1. p. 75. pl. 4, f. 18-20. F.

Type of ferrugineum in Stuttgart Museum ; parasiticum in British Museum; jickelii in Geneva Museum.

Hab. Cape of Good Hope. Knysna River (fermgineum, Krauss). RHodesia. Victoria Falls (Connolly).

Jickeli (1874) placed examples of E. parasitica, which he collected in Abyssinia, in the synonymy of ferruginea, Krs., but the weight of more recent opinion, which in this case appears to me to be correct, is against these species being identical. References to both, as well as to jickelii, Bgt., are given above for purposes of comparison.

Parasitica is recorded from East and North-East Africa; jickelii from Abyssinia. Until more is known of Krauss' species, I prefer to leave it in its original Genus, rather than in Eupera, Bgt., which was constituted for two Brazilian shells. Limosina, Cless., 1872, is preoccupied by Limosina, Macq., in Diptera, 1835.

Genus PISIDIUM, C. Pfeiffer, 1821.

(Syst. Anordn. u. Beschr. deutscher L.-und W --Schnecken, pp. 17, 123.) (=Pisum, Gray, 1847, and Fluminina, Clessin, 1873.)

Type of Genus, P. obliquum (Lam.).

595. Pisidium langlexanum, Melv. \& Pons.

1891 Pisidium langleyanum, M. \& P., A.M.N.H. viii. p. 237. D. $1892 \quad, \quad, \quad$, ix.p.94.pl.5, f.7. F.

Type in British Museum.

Hab. Cape of Good Hope. Port Elizabeth (Langley).

596. Pisidium ovampicum, Ancey.

1890 Pisidium ovampicum, Ancey, Bull. Soc. Mal. Fr. vii. p. 162. D. 1910 ? " c.f. langleyanum, M. \& P., Bttg., Abh. Senckenb. Type-ubi? Naturf. Ges. Frankfurt, xxxii. p. 455. pl. 28,f.19. D.F.

Hab. Ovampoland. Ovambonde (Andersson and Chapman).

British Bechuanaland. Witkop (?langleyanum, subfossil, Schultze).

It appears probable, on geographical grounds, that Böttger's note may refer to $P$. ovampicum, which he seems to have overlooked 
in his article on German South-West Africa. It is, however, impossible to recognise this species, of which I bave been unable to trace an authentic example. It was not figured, and was apparently described from a closed shell, as no mention was made of the hinge or interior of the valves.

Species which have been erroneously attributed to South Africa, or whose names may, for various reasons, be removed from the list of collectible South African varietics.

Ceratoconcha schultzei, Simroth.

1907 Ceratoconcha schultzei, Srth., Zool. Anz. Leipsig, xxxi. p. 794, \&c. D.F.

1910

,

Bttg., Abh. Senckenb. Naturf. Ges. Frankfurt,xxxii.p.433. $N$.

Hab. Cape Peninsula. Cape Flats (Schultze).

Böttger points out that this is the larva of Microdon, Meig., and consequently has no place in Mollusca.

Ennea zanguebarica, Morelet.

1889 Ennea zanguebarica, Morel., J. de C. xxxvii. p. 10.pl.1, f. 7. D.F.

Type in British Museum.

Hab. CAPe of Good Hope. Port Elizabeth (Morelet, laps, cal.).

Owing to an obvious omission in Morelet's article, this species was erroneously attributed to Port Elizabeth. It is a synonym of E. obesa, Gibbons, an East African shell.

\section{Martensia jenynsi (Pfeiffer).}

1845 Helix jenynsi, Pfr., P.Z.S. p. 131. D.

1859 Nanina jenynsi, Pfr., von Mts., Mal. Blätt. vi. p. 211. L.

1897 Trochonanina jenynsi, Pfr., von Mts., D.-O.-Afr. p. 48. N.L.

Type in British Museum.

Hab. Lorenzo Marques. Tette (Peter's).

In 1859 and 1879 von Martens included $M$. jenynsi in the list of shells brought from Tette by Dr. Peters, but in 1897, though a large number of localities are quoted for this species by the same 
author, no mention is made of Tette or any other place within the limits of the present work. It may therefore be advisable to await further confirmation before including it in South African lists.

\section{Rhytida bullacea (Pfeiffer).}

1854 Helix bullacea, Pfr., P.Z.S. p. 53. D.

$1889 \quad$ „, ", Morel., J. de C. xxxvii. p. 19. L.

Hab. Cape of Good Hope. Port Elizabeth (fide Morelet).

An Australian species, erroneously referred to South Africa.

\section{Nanina pisolina, Gould.}

1859 Nanina pisolina, Gld., Proc. Boston Soc. N.H. vi. p. 423. D. $1862 \quad$ ", " , Otia Conch. p. 102. D. 1868 Helix " " $\quad$ Pfr., Mon. Hel. v. p. 54. D.

Hab. "Cape of Good Hope?" (Ringold \& Rodgers).

As the description of this unfigured species is insufticient, no authentic example can be traced, and its original habitat is doubtful, there can be no object in retaining its name among collectible varieties.

\section{Helix comatula, Férussac.}

1821 Helix(Helicella)comatula,Fér.,Tabl.Syst.Moll.pt.3.p.45 (or 41). L. 1874 Helicella comatula, Beck, von Mts., Jahrb. d. Deutsch. Mal. Ges. p. 120 . $N$.

Hab. Cape of Good Hope. On aloes (Delalande).

Nomen nudum.

Helix connexiva, Férussac.

1821 Helix (Helicostyla) connexiva, Fér., Tabl. Syst. Moll. pt. 3. p. 50 (or 46). $L$.

1874 " comexiva, Fér., von Mts., Jahrb. d. Deutsch. Mal. Ges. p. 120. N.

Hab. Cape of Good Hope (Delalande).

Nomen nudum.

Helix dolosa, Férussac.

1821 Helix (Helicostyla) dolosa,Fér.,Tabl.Syst.Moll.pt.3.p.50(or46).L. 1874 " dolosa, Fér., von Mts., Jahrb.d.Deutsch. Ial.Ges.p.120. N Hab. Cape of Good Hope (Delalande).

Nomen nudum. 
Helix ekloniana, Beck.

1837 Helix (Theba) etiloniana, Beck, Index Moll. p. 11. L.

1874 Theba eckloni,Beck,vonMts.,Jahrb.d.Deutsch.Mal.Ges.p.120. N.

Hab. "Afr. aust. Alg." (fide Beck).

Nomen nudum.

Helix fanulus, Pfeiffer.

1856 Helix fanulus, Pfr., P.Z.S. p. 33. D.

Type in British Museum.

Hab. Port Natal (coll. Cuming).

This shell, whose name was intended to be janulus, proved to be the top of an Emnea, and was omitted from Pfeiffer's Nomenclator in 1881 .

Hetix monticola, Beck.

1837 Helix (Bradybana) monticola, Beck, Index Moll. p. 20. L.

Hab. Cape of Good Hope.(fide Beck).

Nomen nudum.

Helix sectilis, Férussac.

1821 Helix (Helicella) sectilis, Fér., Tabl. Syst. Moll.pt.3.p.46(or42). L. 1874 Theba sectilis,Beck,vonMts.,Jahrb.d.Deutsch.Mal.Ges.p.120. N.

Hab. Cape of Good Hope. Caffraria (Delalande).

Nomen nudum.

Ena picturata (Morelet).

1889 Bulimus picturatus, Morel., J. de C. xxxvii. p. 7. pl. 1, f. 4. D.F. 1898 Bulimimus (Rhachis) picturatus, Morel, Stur., S.A. Moll.p.64. L.

Hab. Cape of Good Hope. Port Elizabeth (fide Sturany).

Sturany's locality is erroneous, the species having been described from Mogadoxo, Zanzibar.

\section{Jaminia cylindracea (Da Costa).}

1778 Turbo cylindraceus, Da Costa, Test. Brit. p. 89. pl. 5, f, 16. D.F. 1801 Pupa umbilicata, Drap., Tabl. Moll. Fr. p. 58. D.

1898

M. \& P., Proc, Mal. Soc, iii. p. 184. L.

Hab. Cape Town (fide M. \& P.).

This common European species may possibly exist in South Africa, but I have failed to trace any instance of its actual occurrence there.

Jaminia haploA (Melv. \& Pons.).

1893 Pupa haploa, M.\& P., A.M.N.H. xi. p. 21. pl. 3, f. 7. D.F. 1908

i. p. 77. $N$. 
Hab. Transvaal. Pretoria (fide M. \& P.).

Founded on a single specimen, "seemingly allied to P. pretoriensis" (M. \& P., 1908).

Jaminia psichion (Melv. \& Pons.).

1894 Pupa psichion, M. \& P., A.M.N.H. xiv. p. 93. pl. 1, f. 8. D.F. 1908

1911 , " i. p. $81 . N$.

Hab. Transvaal. Pretoria (fide M. \& P.). Bnp., A.M.N.H. vii. p. $409 . \quad$ N.

It appears advisable to transfer both the foregoing to the list of doubtful species. J. haploa was founded on a single specimen, which can only be regarded as lost, since it has proved quite impossible to trace its whereabouts. In the case of psichion, the type, the only known specimen, is so hopelessly broken that it is quite impossible even to determine to what group of the Genus it belonged. The original descriptions and figures of these minute forms are hardly in themselves sufficient, and the Pretoria District, whence they were recorded, has since been often carefully searched, and yielded only the species mentioned on pp. 180-185. It is probable that, if ever the missing type of haploa or co-types of psichion turn up, they will prove to be identical with some forms already named; meanwhile no useful purpose can be served by retaining them in the list of collectible varieties.

\section{Rumina decollata (Linné).}

1758 Helix decollata, Lin., Syst. Nat., Ed. 10. i. p. 773. D. 1898 Rumina ,, " M. \& P., Proc. Mal. Soc. iii. p. 184. I.

Hab. Cape of Good Hope. Port Elizabeth (coll. Layard).

In the Layard collection were two large examples of this species, found at Port Elizabeth in 1897. There is no record of their having been taken alive, and it is hardly possible that this destructive pest would have gained a footing in the country so many years ago without making its presence felt ere now. It is reasonable to suppose, therefore, that these shells, on which the South African record of $R$. decollata is based, were imported in dead condition, probably in flower-pots, and the name may be expunged from the South African list until, as is to be feared, a second, and more successful, invasion takes place.

\section{Auriculastra pellucens (Menke).}

1830 Auricula pellucens, Mke., Syn. Meth. Moll. p. 131. D. 1848

$$
\text { , Krs., Südafr, Moll. p. } 82 . \quad N \text {. }
$$


Described from South America.

Krauss attributed to $A$. pellucens specimens collected by Wahlberg in Natal. It is unlikely that they were that species; Nevill (Hand List, i. p. 226) assigns them definitely to radiolata, Morel. ; M. \& P. (A.M.N.H. iv, 1899, p. 193) suggest their identity with durbanica, II. \& P., which appears to be inseparable from radiolata.

Auriculastra pusilla (H. \& A. Adams).

1854 Ellobium pusillum, H. \& A. Ad., P.Z.S. p. 8. D.

1555 '́ralia pusilla, H. \& A. Ad., Gen. rec. Moll. iii. pl. 82, f. 8. F. 1898 Auricula pusilla, H.\& A. Ad., M.\& P., Proc. Mal. Soc.iii.p.180. L.

Type in British Museum.

Hab. Natal. Cato's Creek, Durban.

Described from the Philippines. The South African record was based on a single specimen, which appears to have been incorrectly identified.

\section{Planorbis caffer, "Krauss."}

1889 P. caffer, Krs., Morel., J. de C. xxxvii. p. 19. L. 1598 Planorbis caffer, Krs., Stur., S.A. Moll. p. 77. N.

Hab. Cape of Good Hope. Port Elizabeth (fide Morelet).

Sturany points out that the above appellation is probably a lapsus calani, as no such species can be traced in literature. Possibly Morelet's "P." caffer was intended for "Unio" caffer, Krs., which is known from Port Elizabeth, but not included in his list of shells from that district.

Cyclotus natalensis, Pfeiffer.

1861 Cyclotus natalensis, Pfr., P.Z.S. p. 388. D.

$1898 \quad$ " \# Ancey, Bull.Mus.Marseille,i.p.136. N. $1899 \quad$ ", $\quad$ M. \& P., A.M.N.H. iv. p. 193. N.

Type in British Museum.

Hab. Cape Natal (coll. Cuming).

"Cyclotus natalensis, Pfr., has been shown by Ancey to be the Cyclophorus klobukouskii, Morlet, from Tonkin (J. de C., 1884, p. 391), and must therefore be removed from South African lists."

\section{Tropidophora goudotiana (Sowerby).}

1847 Cyclostoma goudotianum, Sow., Thesaurus Conch. i. p. 130. pl. 29, f. 193. D.F. 
The type, in the British Museum, is labelled "Natal," but the species is Madagascan, not South African, and the locality is almost certainly erroneous.

\section{Tropidophora Letourneuxi (Ancey).}

1889 Cyclostoma letoumeuxi,Ancey,Bgt,,Moll.del'Afr.équat.p. 152. D. 1909 Ligatella letoumeuxi, Ancey (=zanguebarica, Pfr., nec Petit), Kob., Abh. Senckenb. Naturf. Ges. Frankfurt, xxxii.p. 78. L L.

Hab. South-East Africa (fide Kobelt).

Recorded from Zanzibar, Nyassa, \&c., but not yet from south of the Zambesi.

\section{Tropidophora zanguebarica (Petit).}

1850 Cyclostoma zanguebaricum, Petit, J. de C. i.p. 53. pl. 3, f. 5. D.F. 1854 „ parvispirum, Pfr., P.Z.S. p. 128. D.

$1889 \quad$ " " $\quad$ " Morel., J. de C. xxxvii. p. 20. L. 1894 " zanguebaricum, Petit (=parvispira, Pfr.), Smith, Proc. Mal. Soc. i. p. $166 . \quad N$.

Type of parvispira in British Museum; zanguebarica-ubi?

Hab. CAPE of Good Hope. Port Elizabeth (fide Morelet).

Zanguebaricum was described from Zanzibar, parvispira from "——? ?"

After careful research, I have failed to trace any authentic instance of the occurrence of this species south of the Zambesi.

Melania histrionica, Reeve.

1860 Melania histrionica, Rve., Conch. Icon. pl. 29, f. 192 . D.F'.

$1878 \quad$ ", " Brot, Conch. Cab. p. 365. N.

$1899 \quad$ ", M. \& P., A.M.N.H. iv. p. 193. N.

Type in British Museum.

Hab. "Cape Colony" (tide Reeve).

M. \& P. remark that $M$. histrionica was described from Cape Colony on the authority of the Cumingian collection. The type appears to be closely allied to, if not a variety of, Pachymelania aurita (Müll.), a West African species whose presence in South Africa appears to require further confirmation. Brot places histrionica in the synonymy of Claviger balteatus (Phil.) from Senegal, which he states is nearly aliied to aurita.

Melanopsis princeps, Lea.

1837 Mclanopsis princeps, Lea, Trans. Amer. Phil. Soc. v. p. 82. pl. 19, f. 74 . D.F'

Hab. CAPE of Good Hope (fide Lea). 
Placed by Brot (Conch. Cab., 1879, p. 411) in the synonymy of Faumus ater (Linn.). Lea's locality is in all probability erroneous.

\section{Theodoxis crepidularius (Lamarck).}

1822 Neritina crepidularia, Lam., Hist. nat. An. s. Vert. vi, 2. p. 186. D. 1848

1899 „ Krs., Südafr. Moll. p. 88. D.N. ,$\quad$ II. \& P., A.M.N.H. iv. p. 194. N.

Hab. Natal Coast (Wahlberg).

Krauss expresses some doubt as to the correctness of Wahlberg's locality. M. \& P. remark, "This species has been found in Ashanti, but we have not met with it at present from South Africa."

\section{List of Principal Authors and Works whose Names have been abbreviated in the Text.}

\section{(a) AUTHORS AND THEIR PUBLICATIONS.}

Adays, H. and A. The Genera of Recent Mollusca. Vols. i.-iii. 1853-58.

Albers, J. C. Die Heliceen. 1st Edition, Berlin, 1850, and 2nd Edition (edited by von Martens), Leipsig, 1860.

Anton, H. E. Verzeichniss der Conchylien ... Sammlung, \&e. Halle, 1839.

Веск, H. Index Molluscorum præsentis Evi, \&c. Hafn. Pp. 1-100, 1837; Remainder, 1838.

Benson, W. H. (Bs.). In Periodicals.

Binney, A. In Periodicals.

Binney, IV. G. The Terrestrial Air-breathing Mollusks of the United States, \&c. Vol. v. Cambridge, Mass., 1878.

Blainville, H. M. D. DE. Manuel de Malacologie et de Conchyliologie. Paris and Strasburg, 1925-27.

Buanford, IV. T. Observations on the Geology and Zoology of Abyssinia. London, 1870.

Böttger, O. (Bttg.). In Periodicals.

Bourguignat, J. R. (Bgt.). Aménités malacologiques. Paris, i., 1856 ; ii., 1860.

Hélixarionidées des Régions orientales ... de l’Afrique. Paris, 1895.

Mollusques de l'Afrique équatoriale, \&c. Paris, 1889.

Déscription de ... Mollusques de l'Égypte, de l'Abyssinie, de Zanzibar, du Sénégal et du Centre de l'Afrique. Paris, 1879.

Les Spiciléges malacologiques. Paris, 1862.

Brard, C. P. Histoire des Coquilles terrestres et fluviatiles qui vivent aux environs de Paris. 1815.

Brown, J. Illustrations of the Conchology of Great Britain and Ireland. London, 1827. 
Bruguik̀re, J. G. In Enc. Méth., q.v.

Burnup, H. C. (Bnp.). In Periodicals.

Chementz, J. H. In Conch. Cab., q.v.

Clessin, S. In Conch. Cab., \&c.

Cockerell, T. D. A. (Ckll.). In Periodicals.

Collinge, W. E. (Cllge.). In Periodicals.

Connolly, M. W. K. In Periodicals.

Craven, A. E. (Crvn.) In Periodicals.

Crawford, J. In litt. ad L. Péringuey.

Cuvier, Baron DE. Leçons d'Anatomie comparative. Paris, 1800.

Le Règne animal distribué d'après son Organisation. Vol. ii. Paris, 1817.

Da Costa, E. M. Historia Naturalis Testaceorum Britanniæ; or, The British Conchology. London, 1778.

Dautzenberg, P. In Pexiodicals.

Deshayes, G. P. Catalogue of the Conchifera or Bivalve Shells in the Collection of the British Museum. London, 1853.

Also in the 2nd Edition of Lamarck's "An. s. Vert.," 1838, and in Férussac's "Histoire Naturelle."

Dillwyn, L. W. A Deseriptive Catalogue of Recent Shells. London, 1817.

Draparnaud, J. P. R. Histoire Naturelle des Mollusques terrestres et Huviatiles de la France. Paris, 1805.

Tableau des Mollusques terrestres et fluviatiles de la France. Paris, 1801.

Ehrenberg, C. G. Symbolæ Physicæ. Berlin, 1828 (plates), and 1831 (text, without pagination).

FÉrussac, Baron A. E. DE. Histoire Naturelle générale et particulière des Mollusques terrestres et fluviatiles. Paris, 1818-52.

Tableaux systématiques des Animaux Mollusques, classés en Familles naturelles... suivis d'un Prodrome Général, \&c. Paris and London. Part i., 1822; Parts ii. and iii., 1821.

Fischer, P. Manuel de Conchyliologie. Paris, 1880-87.

Fleming, J. A History of British Animals. Edinburgh, 1828.

Frauenfeld, G. von (Frnfd.). In Periodicals.

Geoffrox, M. Traité sommaire des Coquilles ... qui se trouvent aux environs de Paris. 1767.

Gibbons, J. S. In Periodicals.

Guelin, J. F. 13th Edition of Linné's "Syst. Nat.," q.v.

Godwin-Austen, H. H. Land and Freshwater Mollusca of India. London, 1882-1910.

The Fauna of British India, Mollusca. London, 1908.

Gould, A. A. Otia Conchologica. Boston, 1839-62.

GraY, J. E. Synopsis of the Contents of the British Museum. London.

Also in "An Expedition of Discovery into the Interior of Africa," by Sir J. E. Alexander. 1838. 
Gray, J. E., and Pfeiffer, L. Catalogue of Pulmonata or Air-breathing Molluscr in the Collection of the British Museum. London, 1855.

Heynemann, F. D. In Periodicals.

Humphrey, G. Museum Calonnianum. London, 1797.

Note--As this work was published anonymously, the nomenclature proposed therein is not accepted.

JEFFreys, J. G. British Conchology. London. Vols. i., 1862, and v., 1869. JickeLt. C. F. Fauna der Land-und Siisswasser-Mollusken Nord-OstAfrika's. Dresden, 1874. (Published in Nova Acta der k-1. Leop.. Carol, Deutschen Ak. der Naturforscher, Vol, xxxvii).

Kовецt, W. In Conch. Cab., \&c.

Krauss, F. (Krs.). Die Sïdafrikanischen Mollusken. Stuttgart, 1848.

Küster, H. C. In Conch. Cab.

LAMArck, J. P. B. DE. Histoire naturelle des Animaux sans Vertèbres. Paris. Vol. vi., Part 1, 1819; Part 2, 1822. (Subsequently edited by Deshayes and Milne-Edwards.)

Layard, E. L. In litt. aủ J. H. Ponsonby.

LEA, I. Observations on the Genus Unio, \&c. Philadelphia, 1834-74.

Lehiann, R. In Periodicals, \&e.

Linné, C. Museum S:æ R:æ M:tis Ludovicæ Ulrieæ Reginæ, \&c. Holmiæ, 1764.

Systema Naturæ per Regna tria Naturæ. Holmiæ. Ed. 10, 1758; Ed. 12, 1767 ; Ed. 13 (by Gmelin), Leipsig, 1788 ; and English translation (by Turton), London, 1801.

Martens, E. von (von Mts.). Conchologische Mittheilungen, als Fortsetzung der Novitates Conchologicæ. Cassel. Vols. i.-iii., 1880-94.

Deutsch-Ost-Afrika. Vol. iv. Beschalte Weichthiere. Berlin, 1897.

Die preussische Expedition nach Ost-Asien. Zool. ii. Berlin, 1867.

Beiträge zur Meeresfauna der Insel Mauritius und der Seychellen. 1880.

"Mollusken" in Passarge's "Die Kalahari." Berlin, 1904.

Maton, W. G., and Racketr, J. In Periodicals.

Melvill, J. C., and Ponsonby, J. H. (M. \& P.). In Periodicals.

Möllendorfa, O. von. (Mildff.) In Conch. Cab., \&c.

Montagu, G. Testacea Britannica, or Natural History of British Shells. London. Parts 1 and 2, 1803; Supplement, 1808.

Montfort, D. DE. Conchyliologie Systématique, \&c. Paris, 1810.

Moquin-Tandon, A. Histoire naturelle des Mollusques terrestres et fluviatiles de France. Paris, 1855.

Morelet, A. Séries Conchyliologiques... de Mollusques terrestres et fluviatiles, \&c. Paris.

"Mollusques" in "Voyage du Dr. Friederich Welwitsch . . . dans les Royaumes d'Angola et de Benguella." Paris, 1868.

Mousson, A. In Periodicals, \&e.

MülLer, O.F. Vermium terrestrium et fluviatilium. Vol. ii. Copenhagen and Leipsig, 1774. 
Nevilu, G. Hand List of Mollusea in the Indian Museum, Calcutta. Part 1, 1878; Part 2, 1884.

Parrkyss, L. In Philippi, Hemmannsen, de.

Pennant, J. British Zoology. London, 1st Edition, 1777.

Pferffer, C. (C. Pfr.). Systematische Anordnung und Beschreibung deutscher Land-und WVasser-Schnecken. Cassel, 1821.

Pfarfer, L. (Pfr.). Catalogue of Auriculida . . . in the Collection of the British Museum. London, 1857.

Monographia Auriculaceorum viventium. Cassel, 1855.

Monographia Heliceorum viventium. Leipsig. Vols. i.-viii. 1847-77.

Monographia Pneumonopomorum viventium. Cassel, 1852; with :3 supplements, abbreviated as Mon. Pneum. ii., iii., and iv.

Novitates Conchologicre. Cassel. Vols. i.-v., 1854-79.

Nomenclator Heliceorum viventium. Cassel, 1878; concluded bỹ s. Clessin in 1881.

Symbolæ ad Historium Heliceorum. Cassel. Vols. i.-iii. 1841-46.

Philippi, R. A. Abbildungen und Beschreibungen neuer oder wenig gekannter Conchylien. Cassel. Vols. i. 1842-45; ii., 1844-47; iii., 1848-51.

Pilsbry, H. A. A Manual of Conchology. 2nd Series. Vols. v.-xx., 1sS91910.

Pollonera, C. ' In Periodicals.

Ponsonby, J. H. In Periodicals. See also Mruvill.

Preston, H. B. In Periodicals.

Pulteney, R. Catalogues of the Birds, Shells... of Dorsetshire. London, 1799.

Quoy, J. R. C., and Garuard, P. (Q. \& G.). Voyage de Découvertes de l'Astrolabe, Mollusques. Paris, 1832.

Reeve, L. (Rve.). Conchologia Iconica. London, 1843̈-65.

Conchologia Systematica. London, 1841-42.

Risso, A. Histoire naturelle des principales productions de l'Europe méridionale. Vol. iv. Paris, 1826.

Semper, C. Reisen im Archipel der Philippinen. Vol. ii, Pirt: Wiesbaden, 1870.

Simrotr, H. (Srth.). In Periodicals, de.

Sowerby, G. B. Conchologia Iconica. London, 1865-78.

Thesaurus Conchyliorum. Vol. i. London, 1847.

Stark, J. Elements of Natural History. Vol. ii. Lainburgh, 1825.

Sturany, R. Catalog der ... südafrikanischen Land-und SusswasserMollusken. (S.A. Moll.). Vienna, 1898 (also published in the Denkschriften der kais. Akad. der Wissenschaften, Wien. Vol. lxvii., pp. 537 et seq.).

Swarson, IV. Treatise on Malacology, ice. London, 1848. 
TAylor, J. W. Monograph of British Land and Fresh-water Mollusea. Leeds, 1894-1912.

Troscinz, F. H. Das Gebiss der Schnecken. Berlin, 1856-62.

Tryon, G. IV. Structural and Systematic Conchology. Philadelphia, $1882,1883$.

A Manual of Conchology. 2nd Series. Vols, i.-iv., 1885-89.

Turton, W. British Fauna. Swansea, 1807.

A Manual of the land and fresh-water Shells of the British Islands. 1st Edition, 1831; and 2nd Edition (by J. E. Gray), 1840.

A General System of Nature, \&c., translated from ... Systema Naturæ. Vol. iv. London, 1801.

\section{(b) SERIALS AND PERIODICALS.}

Abhandlungen Naturwissenschaftlicher Verein von Hamburg-Altona.

Abhandlungen Naturwissenschaftlicher Verein für Sachsen und Thuringen. Halle.

Abhandlungen der Senckenbergische naturforschende Gesellschaft, Frankfurt-am-Main.

Actes de la Société Linnéenne de Bordeaux.

American Journal of Conchology. Philadelphia.

Annalen des Kaiserliches-Königliches naturhistorisches:Hofmuseum, Wien.

A nales des Sciences naturelles. Paris.

Annals and Magazine of Natural History. (A.M.N.H.) Many series, commencing under present title in 1841.

Annals of the Lyceum of Natural History, New York.

Annals of the Natal Museum, Pietermaritzburg.

Annals of the New York Academy of Sciences.

Annals of Philosophy. London.

Annals of the Suth African Museum, Cape Town.

Anzeiger der Kaiserliche Akademie der Wissenschaften, Wien.

Archiv für Naturgeschichte. Berlin.

Archives de Zoologie expèrimentale et générale. Paris.

Atti della R. Accademia delle Scienze, Torino.

Bericht des Offenbacher Vereins fiir Naturkunde.

Bericht iiber die Senckenbergische naturforschende Gesellschaft, Frankfurt am-Main.

Bolletino del Musei . . . delle R. Universita di Torino.

Boston Journal of Natural History.

Bulletin of the Museum of Comparative Zoology, Harvard.

Bulletin du Muséum d'Histoire Naturelle, Paris.

Bulletin de la Société Linnéenne, Paris.

Bulletin de la Société Malacologique de France, Paris.

Bulletin des Sciences par la Société Philomatique, Paris.

Bulletin de la Société Vaudoise, Lausanne.

Bulletin de la Société Zoologique de France, Paris.

Bulletin of the United States National Museum, Washington.

Bulletino della Societa Malacologica Italiana. Pisa.

Conchologist, The. Birmingham. Vols. i. and ii., 1886-88. (Continued as Journal of Malacology.) 
Conchylien-Cabinet, Neues systematisches. (Conch. Cab.) Nuremberg. Founded in 1769 by Martini and Chemnitz, and continued by Schubert and Wagner, Küster, Philippi, von Martens, Brot, Clessin, von Möllendorff, Kobelt, \&c.

Conchological Magazine, The. By Y. Hirase, Kyoto.

Encyclopédie Méthodique. Paris and Liège, 1782-1825.

Jahrbüicher der Deutschen Malakozoologischen Gesellschaft, Frankfurt-amMain.

Journal of the Academy of Natural Sciences of Philadelphia.

Journal of the Asiatic Society of Bengal, Calcutta.

Journal of Conchology. London. Vol. ii., 1879, to present date.

Journal de Conchyliologie. Paris. (J. de C.)

Journal of Malacology. London. Vols. iii., 1894, to xii., 1905.

Journal of the Portland Society of Natural History. Portland, Maine.

Magasin de Zoologie. Paris.

Magazin der Gesellschaft Naturforschender Freunde, Berlin.

Malakozoologische Blätter. Cassel.

Memoirs and Proceedings of the Manchester Literary and Philosophical Society. (Manchester Memoirs.)

Mémoires presentées à l'institut Egyptien. Cairo.

Mémoires de la Société d'Histoire naturelle, Paris.

Mémoires de la Société Zoologique de France, Paris.

Monats-Berichte der königlich-preussische Akademie der Wissenschaften, Berlin.

Nachrichts-Blatt der deutschen Malakozoologischen Gesellschaft, Frankfurt. am-Main.

Nova Acta Academia Cæsarea Leopoldino-Carolina Germanicæ Naturæ Curiosorum.

Proceedings of the Academy of Natural Sciences of Philadelphia.

Proceedings of the Boston Society of Natural History.

Proceedings of the Malacological Society of London.

Proceedings of the United States National Museum, Washington.

Proceedings of the Zoological Society of London. (P.Z.S.)

Quarterly Journal of Conchology. London. Vol. i., 1874-78. (Continued as Journal of Conchology.)

Revue et Magasin de Zoologie. Paris.

Revue Zoologique par la Société Cuviérienne. Paris.

Sitzungs-Berichte der Gesellschaft naturforschender Freunde, Berlin.

Sitzungs-Berichte der Kaiserlichen Akademie der IVissenschaften, Wien.

Transactions of the Entomological Society of London.

Transactions of the Linnean Society, London. (Linn. Trans.)

Verhandlungen der k.k. Zoologisch-Botanischen Gesellschaft, Wien.

Videnskabelige Meddelelser fra naturhistorisk Forening, Copenhagen.

Zeitschrift für Malakozoologie. Hanover and Cassel.

Zeitschrift für Wissenschaftliche Zoologie. Leipsig.

Zoological Journal. London.

Zoologische Jahrbücher. Jena.

Zoologischer Anzeiger, \&c. Leipsig. 



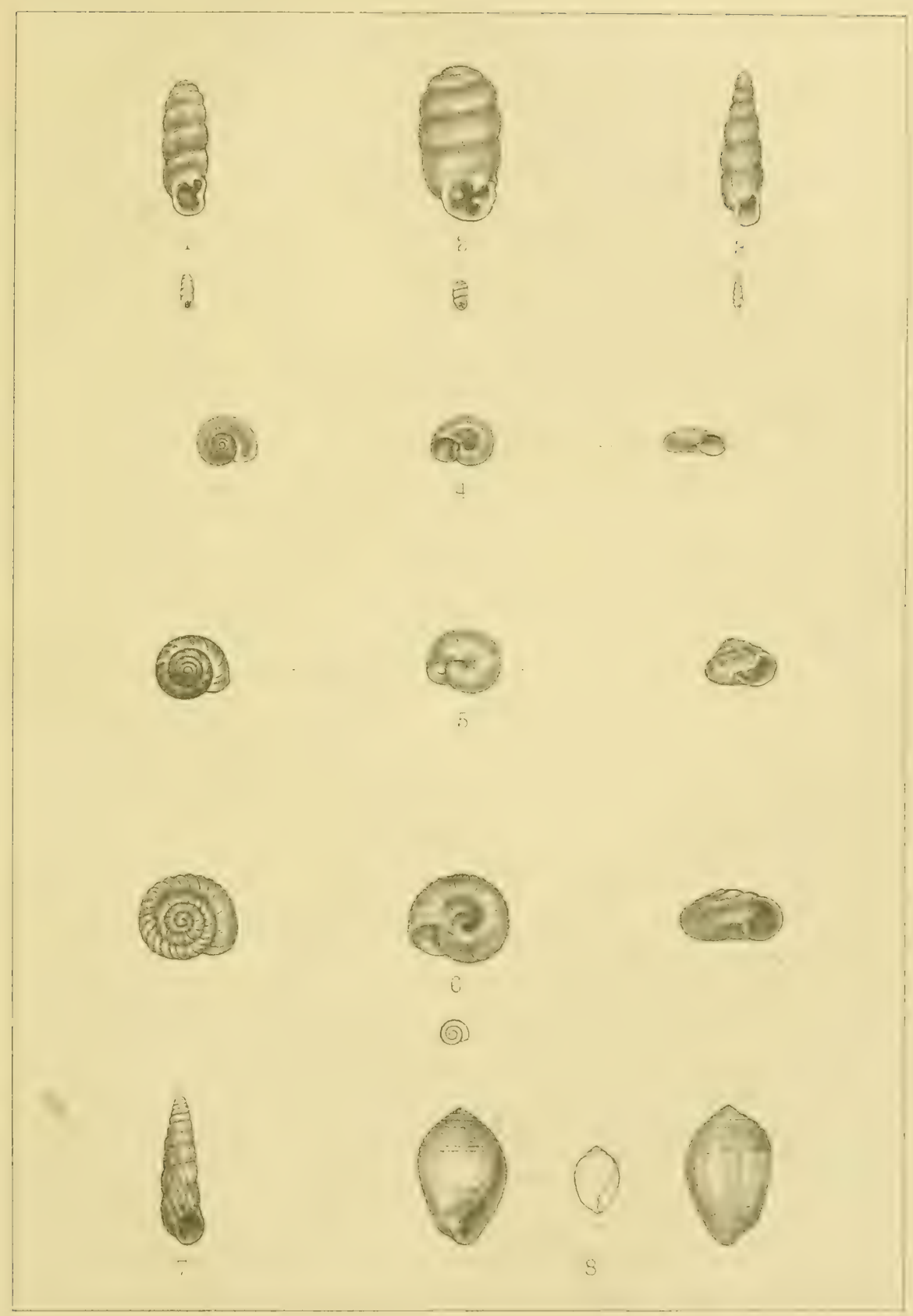

T.B.Stoney del.

West, Nowman lith.

1. Ennea magnolia.

Wnnea rhodesiana.

3. Streptostele herma.

Natalina tarachodes.

5. Trachycystis erythractis. : Trachycystis metallakter.

7. Euonyma gouldi.

. Melampus semiaratus. 



\section{ALPHABETICAL INDEX.}

Names of higher rank than Genera are printed in CAPITALS; Genera and Subgenera, in SMaLI CAPITALS; species in ordinary type (roman); varieties, synonyms, and forms not actually known in South Africa, in italics.

When a valid species is attributed to a Genus other than that in which it was originally placed, the author's name is printed in brackets; but in the case of synonyms and invalid species the original Genus is printed in brackets after the author's name.

The synonyms of the following species, though mentioned in the text, are omitted from the index:-P. draparnaudi; $L$. flavus; $A$. agrestis and lavis; H. lactea and pisana; C. acicula; and $M$. tuberculata.

PAGE

abyssinica, von Mts. Physopsis .. 250

ACAVIDAs, Pilsb. ........... 149

Acavus, de Montfort .......... 160

Aciratina, Lam............... 190

achatina, Lin. (Bulla) ........ 203

ACHATINID $\mathrm{E}$, von Mis. ..... $18 \mathrm{~s}$

ACHATININE, H. \& A. Adams 188

acicula (Mïll.) Crecilioides ...... 206

acinoides, Morel. Melampus...... 226

actinotricha (M. \& P.) Trach..... 133

acuminata (Morel.) Phytia ...... 232

acus, Morel. var. ............ 217

acuta (Miill.) Cochlicella ....... 157

acuta, Lam. Achatina ......... 194

adamsiana, Pfr. Ennea ....... 65

addita, Fér. (Helix.) .......... 156

adspersa, Beck (Helix) ......... 160

advena (Ancey) Crecilioides...... 206

cedigyra, see oedigyra

anea (Krs.) Trach. .......... 133

derope, Alb. .............. 89

Aferulus, von Mts.............. 250

affine, Sow. (Cyclostoma) ........ 255

affinis, Smith, Lanistes. . . . . . . . 258

attinis, Fér. Pedipes .......... 230

afra, Pfr. (Nanina) ........... 117

africa, Brown (Helix) ........ 143

africana, M. \& P. Balea ........ 188

africana (Krs.) Corbicula ..... 277

africana, Krs. Physopsis ....... 249

africana, Krs. Succinea ....... 219

africana, Bgt. Limnæa ....... 23t

africanus, von Mts. (Paludomus) 261

africanus, Bttg. Zonitoides ...... 116

africanus, Lea (Unio) ......... 271

Avrodont, M. \& P........... 127

AFrorhytida, Mlldff. ......... 90

AGNATHA, Miirch. .......... 6.

agrestis (Lin.) Agriolimax ..... 121
PAGE

Agrrolrarax, Mörch.......... 121

alabastrina (Morel.) Paludestrina 265

alabastris, Crvn. (Cyclotus)..... 25 1

albersi (Pfr.) Ennea ........... 66

albida (Krs.) Corbicula ......... 278

albopicta, von MIts. var. ....... 102

alcocki, M. \& P. Trach. ....... 133

alexandri, Gray, Dorcasia ..... 149

alexandrina, Bgt. Limnæa ..... 234

Alexia, Leach............... 232

alqoensis, "MIorel." (Physa) .... 243

aliciæ, M. \& P. Ennea ......... 66

allerian, Gray (Limax) . . . . . . . . 124

alliacea, Jeffr. (Helix) ......... 112

alliaria (Mill.) Polita........... 112

aloicola (M. \& P.) Peltatus ...... $10 \pm$

alpinus, Fér. (Limax) ......... 119

amcena, Morel. (Melania) ....... 261

Amalia, Mloq.-Tand........... 1:0

amarula (Lin.) Melania ... . . . 26.

ambigua, Stur. (Ennea) ....... \$ \$0

ambiguus, von Mts. var. ....... 25s

Ampelita, Beck. . . . . . . . . . . . 99

amplibia, Drap. (Succinea) ..... 219

amphodon, M. \& P. (Pupa) ..... 181

ampliatus (II. \& P.) Kerkophorus 107

ampullacea, Bttg. Achatina...... 191

ampullacea, Stur. (Ennea) ..... \$1

Ampullairia, Lam............. 257

AMPULLARIID $2 \mathrm{e}$, Gray. . . . . . . 257

anceyi, Bgt. (Trochonanina) .... $10^{*}$

Ancylus, Geoffroy .......... 23!

anderssoni, Ancey, Planorbis ... 25.

Andrarion, G.-Aust. .......... 101

angolensis (Morel.) Isidora ..... 24:

Anisoloma, Ancey ............ I I 7

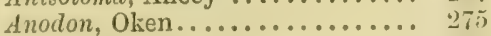

antiquorum, Fér. (Limas) …... 11!)

ApEra, Heynem. .......... i2 
APERID TE, Mlldif. ......... 69

aperostoma, M. \& P. Ennea .... 66

aprica (Krs.) 'Trach. ........... 134

arborea, Ad. it Ang. Succinea.... 220

arborea, Mouss. (Succinea) ...... 220

arboreus (Say) Zonitoides ...... 110

arboricola, Conn. Succinen ...... 220

Arehelix, Albers.............. 161

arenicola (Bs.) Ena ........... 165

arguta, M. \& P. Natalina........ 90

Arion, Fér................ 126

ARIONIDAs, Gray ............ 121

ARIONIN无, Morse .......... 126

ARIOPHAN'TIN E, G. Aust. .... 116

arnoldi, Stur. Ennea.......... 67

arnoldi, Stur. (Livinhacia) ...... 190

arnotti (Bs.) Peltatus .......... 104

aspersa, Miill. Helix ........... 160

ASPIDOBRANCHIA, Schweigger 269

Assemania, Leach ........... 267

ASSEMANIID正, H. A. Ad. .. 267

Assiminea, \&c. ............ 267

astartina (von Mts.) Corbicula .. 278

asthenes (M. \& P.) Peltatus .... 104

ater, Mublf. (Conovulus) _....226, 7

ater (Lin.) Faumus............. 288

aterrima (Gray) Oopelta ....... 124

aulacophora (Ancey) Irach. .... 134

Auricula, Lam. .......... 226-232

Auriculastra, von Mts. ....... 230

AURICULID $₫$, Gray ......... 225

AURICULIN E, Pfr. ......... 230

aurigaster, Layard. Testacella.... 64

auris-lepoxis, M. \& P. Ennea .... 67

aurita (Müll.) Pachymelania .... 287

aurora, Pfr. Achatina ........ 191

Austrocyclus, Ancey ........... 250

avellana, Morel. Melampus ...... 229

badia, Morel. Succinea......... 220

BAleA, Prideaux ............ 188

balteatus (Phil.) Claviger ........ 287

barbara, Bgt. (Helix)........... 158

barrakporensis (Pfr.) Kaliella .... 117

BASOMMATOPHORA, Kfstn. .. 225

Bathyaxis, Ancey ............ 219

bathycole (M. \& P.) Trach....... 134

beccarii, Palad. (Physa) ....... 246

beckeri, Fulton (Stenogyra) ...... 216

beckii, Sow. (Neritina) ......... 270

berthx, M. \& P. Ennea ....... 67

beyrichi (von Mts.) Natalina .... 90

bifasciata, Nev. Assemania ..... 267

bilamellaris, M. \& P. Endodonta 127

binominis, Stur. (Ennea) ....... 82

bisculpta, Smith, Achatina ...... 191

bisculpta (Bs.) 'I'rach. .......... 134

bisulcata (Jick.) Jaminia . . . . . . 178

bloyeti, Brt. Meladomus ....... 260

bloyeti, Bgt. Spatha. ......... 276

boivini (Morel.) Ena........... 165
PAGI

borbonica, Fér. (Helix) ......... 193

borbonica, Desh. (Helix) ....... 157

bormiana, Beck (Achatina) ...... 203

bourguignati, Grandid. (Limic.) .. 190

bourguignati, Ancey (Spathella).. 276

bowkerre, M. \& P. Ennea....... 67

bowkeri (Sow.) Ena ............ 165

bowkeri, M. \& P. Succinea ...... 220

bowkeri, M. \& P. Planorbis ..... 237

bowkeriana, Ancey (Helix) ...... 138

Bradybana, Beck ............ 284

brardiana, Pfr. (Helix) ............ 156

brevis, Krs. var. ........... 267

breweri, Newc. (Hyalina) ......... 116

bridouxiana, Bgt. (Burtoa) ...... 190

brocchii, Ehrn. Isidora......... 245

browningii, Bs. (Helix) ......... 143

brumeus, Drap. (Limax) . ....... 122

Buccinum, Lin......... 206, 234, 262

bulbus (Mke.) Dorcasia ....... 150

Buliminide, Auctt. ......... 163

Buliminus, Ehrn., see Ena, dc.

bulimoides, Moq.-Tand. (Helix) .. 158

butimoides, Swains. (Meladomus) 259

Bulimulus, Leach, see Ena

Bulimus, Brug., see Achatina, Ena,

Euonyma, \&c.

Bulla, Lin................. 203

bullacea (Pfr.) Rhytida....... 97, 283

burchelli (Gray) Ena ........ 166

burnupi, Smith, Apera........ 62

burnupi, M. \& P. Ennea ........ 67

burnupi (M. \& P.) Trach. ...... 135

burnupi (M. \& P.) Ena.......... 166

burnupi, Smith, Achatina ..... 191

burnupi, Walker, Ancylus ...... 242

burnupi, Cllge. Onchidium ...... 224

Bunntia, Walker............. 239

BurtoA, Bgt. .............. 189

cacuminata (M. \& P.) Euonyma 213

Cecilioides, Fér. ............ 205

Cacilionella, Bgt. ................ 205

caffer, Krs. (Bulimus) ......... 175

caffer, Krs. Ancylus ........... 239

caffer, "Krs." (Planorbis) ...... 286

caffer (Küist.) Melampus ........ 226

Cafferia, Simpson .......... 271

caffra (Krs.) Cafferia ............ 271

caffra (Fér.) Natalina ......... 90

caffra, Beck, var. .............. 255

caffrula, M. \& P. Natalina ...... 91

cairnsi, M. \& P. Ennen.......... 68

calaharicus (Bttg.) Leucoch. .... 177

calcarea (Sow.) 'Tropidophora.... 253

caledonensis, G.-Aust. Peltatus .. 105

caledonensis, Chaper (Hydrobia).. 266

callista, M. \& P. Ennea ....... 68

caloglypta, M. \& P. Curvella ... 208

calopasn, M. \& P. Ennea ..... 68

calorama, M. \& P. 'I'rach. ...... 135 


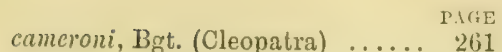
campestris, "Krs." (Limax) .... 120 candidula, Morel. Ennea....... 68 capense (Krs.) Sphxrium...... 280 capense (Pfr.) Phortion ....... 129 capensis (Krs.) Milax ........ 120 capensis (Kurr) Jaminia ........ 185 capensis, Pollon. Oopelta ....... 125 capensis, Mke (Helix) ........ 129 capensis, Alb. Achatina........ 20. 203 capensis, Walker, var. ........ 240 capensis, Krs. (Truncatella) ...... 266 capillacea, Morel. (Physa) ..... 216 capillata, Frnfd. Vivipara ..... 260 capsula (Bs.) Peltatus .......... 105 carinifera (M. \& P.) Ena ........ 166 caryatis, M. \& P. Ennea ....... 68 Cissiduli, Fér............ 231 castaneus, Mühlf. Melampus .... 229 catarractie (M. \& P.) Curvella .. 208 catonis (M. \& P.) Auriculastra .. 231 cavannc, Paul. (Hyalina)........ 112 cellaria (Müll.) Polita ......... 113 centrifuga, M. \& P. Trach. ..... 135 Ceratoconcha, Srth. ......... 28. cerea, Pfr. (Helix) ............ 92 cernua (von Mts.) Dorcasia ...... 150 cestus, 13s. (Helix) ............ 156 chaplini, M. \& P. Natalina ...... 92 chapmani, M. \& P. (Stenogyra) .. 211 chapmanni, Ancey (Sculptaria) .. 131 charybdica, M. \& P. (Pupa)...... 181 charybdis (Bs.) Trach. ......... 135 chemnitziana, Pfr. (Achatina) .... 203 Chersina, Humphrey ......... 203 Chilogymnus, Jouss. ........... 218 Chlamydephorus, Binn. ....... 62 Chondrocyclus, Ancey ....... 251 Choristoma, de Christ. \& Jan. ... 266 chrysoprasinus (M. \& P.) Microkerkus .............. churchilliana, M. \& P. Achatina cimolia, M. \& P. Ennea ........ cinctus, Dum. (Limax) .......... cinereus, Lister (Limax) ........ cingulatus (M. \& P.) Kerkophorus cinnamomea, M. \& P. Achatina Cionclla, Jeftr.

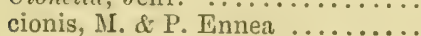
citrinus, IVesterl. (Limax) ...... CLAUSILIID E, B. B. Woodw. .. claustraria, M. \& P. Ennea ..... . clavulata, Morel. (Physa)........ Cleopatra, Troschel ............ coacta (Meusch.) Melania ...... coagulum (von Mts.) Trigonephrus Cochlicella, Fér. . . . . . . . . . . Cochlitoma, Fér. ............ Cochlohydra, Fér. ............ CCELIAXIN ⿷, Pilsb. ......... Ceelaxis, Ad. \& Ang. .........
PAgt:

cunopictus (Hutt.) Leucoch. .... 177 conotera (M. \& P.) Natalina .... 92 corneyensis, MI. \& P. Natalina .. 92 carvulea, Küst. qar. .......... 220 collaris (Pfr.) Sculptaria ........ 131 collieri, M. \& P. (Ennea) ........ 76 columnella, M. \& P. Ennea ... 69 comatula, Fér. (Helix) .......... 28: comburens, M. \& P. Tropidophora 253 companyoi, Nunnely (Limax) .... 119 compta, M. \& P. Isidora ........ 244 congellana, Krs. (Helix) ........ 108 conisalea (1I. \& P.) Trach. ...... 136 connexiva, Fér. (Helix) ......... 283 connollyi, M. \& P. Ennea........ 69 connollyi, Prest. Thapsiella .... 104 connollyi, M. \& P. Trach. ...... 136 connollyi, Prest. Succinea ...... 220 connollyi, Prest. Achatina ...... 192 connollyi, TValker, Ancylus...... 242

Conovulus, Lam. ............. 226 consobrina, Ancey, Ennea ...... 70 consobrina (Caill.) Corbicula . . 277, 279 contorta (Mich.) Isidora ........ 211 Conulinus, von Mts. ......... 164 conulus (Rve.) Ena ........... 167 convexiusculus (Pfr.) Chondr. .. 251 cor, Lam. (Cyrena) ........... 279 Corbicula, Mlegerle von Mühlfeld. 276 Coretus, Adauson ........... 236 Corilla, H. \& A. Ad. ............ 131 comalic, Pini (Limax) ......... 119 cornea, Morel. (Physa) .......... 249 corneus (Pfr.) Kerkophorus ...... 108 corrugata, Prest. Jaminia ...... 179 corticinus, Morel. Melampus .... 229 cosmia (Pfr.) Natalina ......... 92 costata, Prest. var. ........... 26. costulatus, Krs. Planorbis ..... 235 cotyledonis (Bs.) Peltatus ...... 105 couropa, Less. (Achatina) ...... 193 coxi, Prest. Helicarion .......... 100 coxi, Prest. Trach. ........... 136 crassa, von Mts, var. . . . . . . 190 crassidens, Pfr. Ennea......... 70 crassilabris, Crvn. Ennea ..... 70 craterodon, M. \& P. Ennea..... 70 craveni, Ancey (Physa) ......... 248 craveni, Stur. ('hysa) .......... 248 crawfordi, M. \& P. Ennea ...... 71 crawfordi (M. \& P.) Zingis ...... 99 crawfordi, Morel. Achatina ...... 192 crawfordi (MI. \& P.) Opeas ...... 211 crawfordi, M. \& P. Planorbis .... 235 crawfordi, Brot, Melania ....... 263 crawfordiana (M. \& P.) Jaminia.. 179 crepidularius (Lam.) Theodoxis .. 288 crispula, M. \& P. Ennea ....... 71 croslyi, Bnp. Curvella ........... 208 crossleyana, M. \& P. Ennen .... 71 crotallaria, Schum. (Achatina) .. 210 


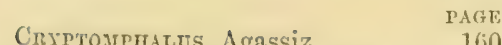

cryptoplax (M. \& P.) Janinia .... 179 crystallina (M. \& P.) Euonyma .. 213

crystallina (Miill.) Vitrea ........ 111 cupido, M. \& P. Zonitoides ...... 117

Convelua, Chaper ............ 207 custodita, M. \& P. (Pupa)......... 181

cyamus, Phil. (Unio) .......... 271 ('yclas, Bruc. .............. 280

CYCLOPHORID E, Fischer $\ldots . .250$ CYCLOPHORIN E, Gray........ 250

Cyclophoropsis, Dautzenberg .... 250 Crchoruorus, de Montfort ..... 250 Cyclostoma, Lam. ........ 251, \&c. Cyclostomatida, Auctt. ......... 252 Cycloiss, Guilding ......... 251, 2 cylindrica, Stur. var. ......... 77 cylindracea (Da Costa) Jaminia .. 284 Cyrena, Lam., see Corbicula cyrences, Nunn. (Limax) ........ 119 CYRENIDE, Gray ......... 276 cyrtonota, Bgt. (Physa)........ 218

dadion (Bs.) Jaminia............ 179 dxdalea, M. \& P. Envea ....... 71 dakaënsis, Stur. Limnøa ....... 233 dakaënsis, Stur. Succinea ..... 220 damarana, Bttg. Limnrea........ 233 damarensis (Ad.) Sculptaria .... 131 damarensis (Ad.) Ena ......... 167 damarensis, Pfr. Achatina ...... 192 damarica (Áncey) Jaminia ..... 179 dammarensis, see damarensis darglensis, M. \& P. Ennea ...... darglensis, Bnp. var........... debaizei, Bgt. Limna. .......... decollata (Lin.) Rumina ........

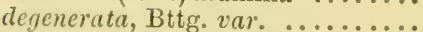
delalandei, Pfr. Succinea ...... delicata, M. \& P. Zingis ........ delicatula, Pfr. Ennea ........... densestriata, Prest. (Vivipara).... clensestriata, Conn, var, ........ diaphana (Krs) Isidora........... differens, Stur, (Ennea) ........ dimera (M. \& P.) Ena ........... dimidiata, Smith, Achatina .... dioryx, M. \& P. I'rochozonites ... discrepans, Stur. var. ......... distincta, M. \& P. Ennea ......... DITREMA'TA, Fischer \& Crosse docimasta, M. \& P. Ennea ..... dokimasta, M. \& P. (Ennea) .... dolichoskia, M. \& P. Ennea .... dolosa, Fér. (Helix) ............

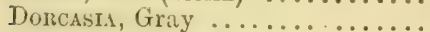

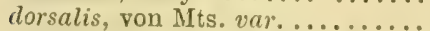
drakensbergensis, M. \& P. Ennea drakensbergensis (Smith) Ena ... drakensbergensis, M.\& P.(Achatina) draparnaudi (Beck) Polita ......
PACiv

(Stur.) Ena ........... 16!

clubiosus, Walker, var. ......... 241

dumeticola (Bs.) Natalina ...... 93

dunkeri, Pfr. Ennea ........... 73

durbanense, Stur. Opeas ....... 212

durbanensis, Stur. (Ennea) ...... 70 dubanica, M. \& P.(Auricula)... 231 dysorata (M. \& P.) Jaminia..... 180

Eburnea, Mouss. .............. 164

cburnea, Hartm. (Helix) ....... 111 ectima, M. \& P. Trach. ....... 130 ckloniana, Beck (Helix) ....... 284 Illa a, Hutton ................ 98 ELASMOGNATHA, Mörch. .... 219 elatior, von Mts. varr......... 103, 259 elevata, Bnp. Curvella ........ 208 elizabethensis, M. \& P. var....... 181 elliptica, M. \& P. Ennea ....... 73 ellipticus, von Mts. Lanistes .... 257 Ellobiince, H. \& A. Ad. ....... 225 Ellobium, H. \& A. Ad.......... 286 elongata, Fér. (Helix) ......... 221 elongata, Stur. var. .......... 67 emicans, M. \& P. Segmentina .. 238 emini, von Mts. var........... 190 Eva, Leach.............. 163 Endodonta, Albers .......... 127 ENDODONTIDE, Crosse ...... 127 endoplax, M. \& P. (Pupa)........ 181 ENID AE, B. B. Woodw. . . . . . 163 ENNEA, H. \& A. Ad. .......... 64 LNNEINA, Kob. ........... 64 epetrima (AI. \& P.) Trach. ...... 136 epixantha, Pfr. (Helix) ......... 157 equeefensis, Walker, Ancylus .... 242 cratina, M. \& P. (Helix) ....... 143 erythractis, Conn. Trach. ..... 136 Erpetrometra, Lowe ........... 266 eshowensis, M. \& P. Ennea .... 73 euconuloides, M. \& P. Kaliella .. 117 Euhyalinia, Albers ..........113,4 EULAMELLIBRANCHIA, Pels. 271 eulimoide (Prest.) Opeas ....... 212 eulimoides (Gray) Zootecus....... 218 Eulota, Hartmann .......... 156 eumacta (M. \& P.) Natalina .... 93 EUONYMA, M. \& P............ 213 Euparypha, Hartmann ......... 162 Eupera, Bgt. ............... 281 cuphratica, Lam. (Cyrena) ..... 279 euschemon, M. \& P. (Ennea) ... 70 custilbus, Bgt. (Zonites) ....... 112 euthymia, M. \& P. Ennea ..... 73 EU'IHYNEURA, Spengel. . . . . . 62 exarata, Krs. Succinea ......... 221 excedens, Stur, var. .......... 75 excentrica, Sterki, Vallonia...... 159 eximia, M. \& P. Ennea......... 74 cximia, Bgt. (Physa) .......... 250 cxserta, von Mts. var. ......... 234 
exsertus, M. \& P. Chondr. ..... P $25:$ exspectata, Mouss. var. ....... 168

fallax, Say (Bulimus) ........ 177

funulus, Pfr. (Helix) ........... 284

farquhari, M. \& P. Ennea ..... 74

farquhari, Bnp. Endodonta ..... 128

farquhari (M. \& P.) Trach. ...... 137

farquhari (M. \& P.) Jaminia .... 180

farquhari, Walker, var. ....... 210

fasciata, Küst. var. ......... 228

fasciata (Krs.) Assemania....... 267

fasciatus, von Mts. Urocyclus .... 123

fasciatus, Nilss. (Limax) ....... 126

Faula, H. \& A. Ad. ......... 18.

Fauxulus, Schauf. .......... 185

Fennissia, Wallier.......... 24:

ferruginea (Lea) Cleopatra ...... 261

ferrugineum (Krs.) Sphærium ..... 280

ferrussaci, Dkr. (Bulimus) ..... 174

fischeriana, Bgt. (Physa) ....... 246

tissidens, Bttg. Unio .......... 274

flavescens, Cllge. Oopelta ..... 125

flavescens (Kfstn.) Urocyclus .... 123

flavus, Lin. Limax............ 118

florentiæ, M. \& P. Hypolysia .... 210

fluminalis (Müll.) Corbicula...... $27 \mathrm{~s}$

Fluminina, Cless. ........... 281

fatida, Stark (Helix).......... 112

fontana (Krs.) Jaminia .......... 180

fonticola, Desh. (Pupa) ....... 185

fontinalis, Walker, Ancylus..... 242

foriclusa, M. \& P. Ennea ........ 74

formosa, M. \& P. Ennea ........ 74

forskali, Ehrn. Isidora ........ 245

fossaria, Mont. (Helix) ......... 234

foveolata (M. \& P.) Tropidophora 253

frivaldskyana, Zeleb. (Corbicula) 279

frustillum, M. \& P. (Pupa) ..... 181

fryana (Bs.) Jaminia ......... 186

fulgurata, Pfr. var. .......... 201

fulica (Fér.) Achatina ......... 193

fulva (Brug.) Achatina .......... 194

fuscata, Lam. (Cyrena) ...... 279

fuscicolor (M. \& P.) Kerkophorus 108

fuscolabris, von Mts. (Achatina) .. 188

fusca, Phil. (Auricula) ......... 229

fuscus (Müll.) Arion ......... 126

gagates (Drap.) Milax ......... 120

Gallandia, Bgt., see Peltatus, \&c. GASTROPODA, Cuv. ......... gaulus, Gld. (Aneylus) ......... gauritziana, Krss. (Cyrena) ..... genialis, M. \& P. Ennea ....... GEHYDROPHILA, Fér. ........ genulabris, von Mts. (Helix) .... Geomalacus, Allman .......... gibbonsi (Binn.) Apera ....... gibbonsi, Nelson, Planorbis ...... giraudi, Bgt. (Bulimus) .......
PHGE

Glandina, Schum. ......... 163 glanvilleana (Ancey) Jaminia .... 186 glanvilleana (Ancey) Trach. .... 137 glaphyra, Say (Helix) ........ 113 glaucocyanea, M. \& P. (Subulina) 217 glebaria, M. \& P. Trach......... 137 globosa (M. \& P.) Curvella ...... 208 globosa, Morel. Physopsis ..... 250 globosus, "Müll." (Helix)........ 151 globularis, "Morel." (Physopsis) 250 globulosa, Jick. var. .......... 181 globulus (Müll.) Trigonephrus .. 153 glutinosa, Pfr. Achatina ....... 194 gokweanus (Bttg.) Crcilioides .. 207 gordonensis, M. \& P. vav. . . . . 239 goudotiana (Sow.) Tropidophora .. 286 gouldi, Pfr. Ennea............ 75 gouldi, Conn. Euonyma ....... 214 gradata, M. \& P. (Physa)........ 245 grandidieri, Pilsb. var. .......... 190 granifer (Mouss.) Melampus .... 229 granulata, Pfr. Achatina ....... 194 granulata, Krs. var. .......... 204 granulosa, Cllge. Oopelta....... 125 granum, Morel. (Assemania) ... 268 greyi, da Costa, Achatina........ 195 griqualandica (M. \& P.) Jaminia 182 grisea, Gmel. (Helix)........... 160 gwandaensis, Prest. Streptaxis .. 64 gypsinus (M. \& P.) Trigonephrus 151 Gyraulus, Agassiz ........... 237

Hapalus, Alb. ........... 207 haploa, M. \& P. (Pupa).......... 281 harpula, Fér. (Physa) ........ 245 hartmanni, von Mits. (Spatha) .... 275 hartvigiana (Pfr.) Trach........ 137 hartvigiana (Pfr.) Tropidophora .. 254 hauttecouri, Bgt. Unio .......... 274 haygarthi, M. \& P. Zingis ..... 99 Helicarion, Fér. ............ 109 HELICARIONIN E, G.-Aust. . . 90 Helicella, Fér.. see Polita, \&c.

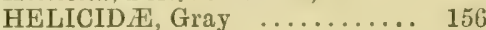
Helicodonta, Fér. .......... 132 Helicogena, Fér. ............ 161

Helicopsis, Beck............ 141

Helicostylı, Fér.............. 283 HELIX, Lin. ............. 160 hemprichi, Ehrn. (Isidora) ..... 245 herma, Conn. Streptostele ..... 89 hermanni, Bttg. Planorbis ..... 236 hevstoni, Coop. (Limax) ....... 121 hickeyana, M. \& P. Ennea ...... 75 hidalgoi (Gass.) Assemania ...... . 268 hiemalis, Drouet (Geomalacus) . . 127 IIijubiu, G.-Aust. .......... 250 himerothales, M. \& P. Ennea .... 75 histrionica, Rre. (Melania) ..... 287 HOLOGNA'THA, IV. G. Binn. .. 98 hortensis, Penn. (Helix) ....... 160 


\begin{tabular}{|c|c|}
\hline & PAGE \\
\hline hottentota (Grry) Ena.......... & 169 \\
\hline hottentota (M. \& P.) Trach. .... & 138 \\
\hline dgonn (Bs.) Peltatus.......... & 105 \\
\hline hudsonic, Bs. (Helix) ......... & 105 \\
\hline huttonice, "Bs." (Vitrina) ...... & 105 \\
\hline hyalina, M. \& P.var. ......... & 89 \\
\hline Hyalinia, Charp. ............ & 111 \\
\hline Hydrobia, Hartmann. . . . . . . . & 265 \\
\hline Hydroliidee, Auctt. ........... & 265 \\
\hline HYDrocent, Parr. . . . . . & 269 \\
\hline HYDROCENID 玉, Bgt. . & 269 \\
\hline Hydrolimax, Malm. .......... & 122 \\
\hline hygapanus, Bttg. Unio .......... & 274 \\
\hline HYGROPHILA, Fér. . . . . . . . & 232 \\
\hline hypochlora, M. \& P. (Nanina) .... & 105 \\
\hline HYPOLYSIA, M. \& P. ........... & 209 \\
\hline hypsoma, M. \& P. Ennea ..... & 75 \\
\hline Hyridella, Swainson .......... & 274 \\
\hline
\end{tabular}

immaculata, Lam. Achatina .... 195 impervia, M. \& P. var. ........ 65 inclara (Morel.) Trach. ....... 138 incommodus, Hutt. (Limax) ...... 126 inconspicua, Bnp. var. ........ 184 indotata, Rve. Achatina ...... 196 infans, Crvn. Ennea.......... 76 infrendens, von MIts. Ennea .... 76 ingens, Ancey, var. ......... 259 ingens, Stur. Ennea ......... 76 inhambanica, von Mts. (Melania) 264 inhluzana (M. \& P.) Natalina .. 94 inhluzaniensis, Bnp. Endodonta 128 inops, Morel. (Helix) ........... 138 insignis, M. \& P. Natalina ..... 94 instabilis, Stur. Ennea ....... 76 insularis (Pfr.) Tropidophora .... 254 intermedius, Normand, Arion .. 127 intradentata, Bnp. (Pupa) ..... 183 inunctus (M. \& P.) Kerkophorus. . 108 iota (M. \& P.) Jaminia ... . . . . . 182 Iridina, Lam. ............. 275 irrorata, Zglr. (Helix) ........ 129 Isidora, Ehrn. .............. 243 isipingöensis, Stur. Ennea ..... 77 isipingöensis (Stur.) Chondr. .... 252 isomerioides, M. \& P. Dorcasia .. 152

jacobi, da Costa, Achatina ...... 196 Jamria, Leach in Risso ........ 178 janulus, Pfr., see fanulus japonicum, Pilsb. Pupisoma .... 158 jejunus, M. \& P. (Buliminus) .... 173 jenynsi (Pfr.) Martensia ........ 282 jickelii, Bgt. Eupera ........... 281 jickelii, Krs. (Physa) ........... 246 johannesburgensis, M. \& P. Ennea 65,77 jouberti, Bgt. (Burtoa) ......... 190 jouberti, Bgt. Limnæa ......... 234 juxtidens, M. \& P. Ennea ...... 77

KALiella, Blanford ........ 117
Kiriophorus, G.-Aust. . . . . P. 107

lierœa, M. \& P. (Pupa) ......... 181

kirki, Gray, Urocyclus ......... 123

klobukowskii, Morlet, Cyclophorus 286 knorri (Récluz) Theodoxis ...... 270 knysnaensis (Pfr.) Natalina...... 94 linysnaensis, Prest. Helicarion .. 100 knysnaensis, Prest. Trach. ..... 138 knysnaensis (Krs.) Assemania.... 268

kosiensis, M. \& P. Ennea....... 77 kransii, Rve. (Achatina) ....... 204 kraussi, Pfr. Ennea ........... 78 kxaussi (Pfr.) Natalina .......... 94 kraussi (Pfr.) Metachatina ...... 188 liraussi, Rive, v(1). ........... 201 kralissiana (Pfr.) Tropidophora .. 254 kraussianus (Heynem.) Urocyclus 123 kraussii, Küst. (Auricula)....... 231 kraussii, H. \& A. Ad. (Limax) . 120 Kryniclia, Fischer .......... 122 kunenensis, Mouss. Unio........ 274 kurri, Krs. (Pupa) . . . . . . . . . . 185 kurri, von Mts. var. ........... 221 küsteri (Krs.) Melampus ........ 227 kynganica, Bgt. (Cleopatra)...... 261

labrella (Desh.) Cassidula ..... 231 labyrinthea, M. \& P. Ennea .... 78 labyrinthica, "M. \& P." Ennea .. 78 lactea, Müll. Helix .......... 161 læocochlis, M. \& P. Euonyma .... 214 lævis (Miill.) Agriolimax ....... 122 Laimodonta, Nuttall ......... 230 lamarcliana, Pfr. (Achatina) .... 198 LAMELLIBRANCHIA, de Blainv. 271 lamellosa, Roth (Physa) ....... 246 lanceolata (Pfr.) Euonyma ...... 214 langleyanum, M. \& P. Pisidium .. 281 Lanistes, de Montfort ......... 257 laticostata, M. \& P. Trach...... 139 laurenti, Bgt. Limnæa .......... 2 234 lavigeriana, Bgt. (Burtoa) ..... 190 lavigeriana, Bgt. Limnæa ...... 234 layardi, Ancey (Ennea) ...... 68 layardi (M. \& P.) Ena ......... 169 layardi (Bs.) Jaminia .......... 182 layardi, Pfr. Achatina .......... 196 layardi, Ad. \& Ang. Coeliaxis .... 219 lechaptoisi, Ancey (Achatina) .... 198 ledoulxi, Bgt. (Rachis) ......... 173 Ledoulxia, Bgt. ........... 101, 102 lepidum, Conn. Opeas ......... 212 leppani, Stur. Ennea........... 78 letoumeuxi (Ancey) Tropidophora 287 Leucochila, Alb.............. 176 LEvCOCHILOIDES, Pfr. ......... 176 leucochilus, M. \& P. Planorbis .. 236 leucospira (Pfr.) Kerkophorus .... 103 levis, Conn. var. ............ 216 ligata (Müll.) Tropidophora...... 255 Ligatella, von MIts. . . . . . . . . 253 


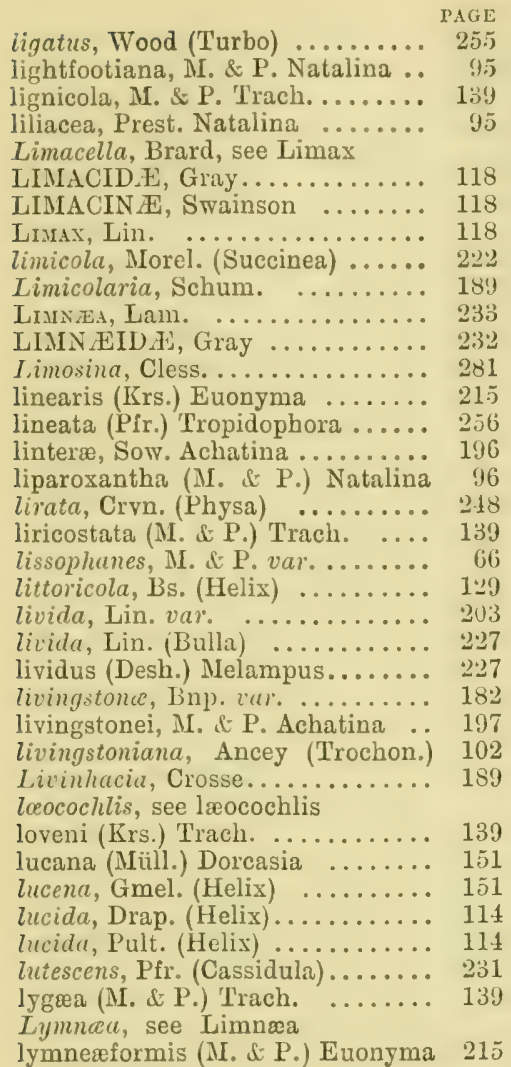

mabillei d mabillianus, Baud (Geo.) 127 machachensis, Smith, Achatina.. 197

Macrocrcloides, von Mits. ..... 89

Macroogona, Pilsb. .......... 149

maculata, Plate, Onchidella .... 2.25 magilensis, Crvn. (Cyclophorus) .. 251 magnolia, Conn. Ennea ....... 78 maitenguensis, Stur. Spatha .... 275 Marzania, Bgt. ............. 250 majubana, Conn. Curvella ..... 209 mamboiensis, Smith (Buliminus).. 165 mamillata (Crvn.) Subulina..... 210 mareotica, Parr. (Physa) ....... 245 margarettæ, M. \& P. Ennea...... 79 marginata, Pini (Limax) . . . . . . 121 marginatus (Say) Leucoch. ...... 177 marix, M. \& P. Ennea ......... 79 marinella, "King" (Auricula).... 230 Marindel, King............ 230 maritzburgensis, M. \& P. Ennea.. 79 maritzburgensis (M. \& P.) Ena .. 170 Martensia, Semper ........... maura (Heynem.) Veronicella..... 2.. 2.3 mauritiana, Lam. (Achatina) .... 1:;: mauritziana, de Blainv. (Peronia) 221 maurus, Q. \& G. (Limax)........ 120 maximus, Lin. Limax ......... 119 mcbeani, M. \& P. Opeas ....... 21: mcbeaniana, Bnp. Ena ....... 169 mebeaniana (H. \& P.) Jaminia .. 187 HeLadoyos, Swainson ........ 257 IELAMPIN E, H. \& A. Ad. .... 225 Melampus, de Montf. ......... 226 melanacme (Pfr.) Ena ......... 170 JELAVIA, Lam. ............ 262 MELANIIDE, d'Orb. ........ 262 MELANIIN E, H. \& A. Ad. .... 262 melanopneumon, Bergh (Onchidium) 225 Melanopsis, Fér. ........... 287 melvilli, G.-Aust. Kerkophorus .. 109 melvilliana, Ancey (Sculptaria) .. 131 menkeana (Pfr.) Ennea ........ 79 menkeanum (Pfr.) Phortion...... 129 meridionalis (Pfr.) Ena......... 170 meridionalis, M. \& P. var. ..... 106 Mesembrinus, Albers ........... 165 Mletachatina, Pilsb............ 188 metallakter, Conn. Trach. ...... 140 metuloides (Smith) Ena ....... 171 micans, Pfr. (Bulimus) ......... 214 Microdon, Meig.............. 282

Micrónerisus, G.-Aust.......... 111 microscopica (Krs.) Trach. . . 140, 159 Mrcrostele, Bttg............. 178 microstriata, Prest. Trach...... 140 microthauma, M. \& P. Ennea .. so Mistx, Gray ............. 121 miniata, lirs, var............. minimus, M. \& P. (Cyclophorus) .. 252 minimus, Srth. (Arion) ......... 127 j9S, 168, 175, minor, Pfr. var'. ....... 205, 252, 255 minor, Bttg. var. ............ 150 minor, Pollon. Oopelta ....... 125 minor, Bs. vart. ........... 182, 25\% minor, Fér. iar.............. 19s minor, Küst. var. .......... 227 minusculus (Mouss.) Leucoch. .. 177 minuta, Morel. Ennea ......... 80 minuta, Say (Helix) .......... 159 minuta, Drap. (Limnæa) ........ 23t minythodes (M. \& P.) Zingis .... 99 modesta, Conn. Curvella ....... 209 modesta, Lea (Spatha) ......... 275 mollerii, Pollon. (Ariōn) ......... 127 MOLLUSCA, Cuv. ........... 62 monile, Rve. (Auricula).......... 227 montana, M. \& P. Ennea........ 80 monticola, Beck (Helix)......... 28. 28 mooiensis, Walker, Ancylus .... 241 moreleti, Desh. (Melania) ........ 263 
morrelli, Prest. Cleopatra...... 262 mossambica, Branczik (Achatina) 198 mossambicensis (Ptrs.) Cafferia .. 273 mossambicensis, "Pfr." Martensia 102 moussoni, von Mts. Succinea .... 221 movenensis, Stur. (Buliminus).... 165 mozambica, Bgt. (Trochomorpha) 102 mozambicensis (Pfr.) Ena ...... 171 mozambicensis (Pfr.) Martensia .. 109 multidentata, Stur. Ennea ..... 80 munda, Bs. (Helix) .......... munita, M. \& P. Ennea ........ IIutela, Scopoli .............. MUTELID E, Gray ........... myosotis (Drap.) Phytia .......

namaquanum (von Mits.) Phortion 130 namaquensis (M. \& P.) Trigonephrus 155 namibicus (Bttg.) Zootecus ...... 218 Nanina, Gray......... 105, 282, 283 nanus, Walker, var. .......... 239 natalensis, Cllge. Apera ....... 63 natalensis, Crvn. Ennea ....... 81 natalensis, Morel. (Ennea) ..... 82 natalensis (Pfr.) Peltatus ........ 106 natalensis (Krs.) Kerkophorus .. 109 natalensis (Krs.) Ena ......... 171 natalensis, Pfr. Achatina ....... 197 natalensis (Bnp.) Euonyma .... 215 natalensis (von Rapp) Veronicella natalensis, "Krs." (Limax) ...... natalensis, Krs. Limnæa ........ natalensis, Krs. Planorbis ...... natalensis (Krs.) Isidora ........ natalensis, Pfr. Cyclotus ........ natalensis (Rve.) Theodoxis...... ratalensis, Lea (Unio) .......... natalensis, Lea (Spatha) ....... natalensis, Krs. Corbicula ...... Natalia, G.-Aust. ............. natalica, Bgt. (Physa) ......... Natalina, Pilsb. ............ ratalis, "Krs." Planorbis ...... navigioliformis, Lea (Unio) ....... Nerita, Lin. ........... 255, 264 NERITID $\mathrm{E}$, Fleming ........ 270 Neritina, Lam. ............ 270 nigra, Phil. (Auricula) ......... 230 nigricolus, Tate (Limax) ....... 121 nigropunctata, Mörch, Oopelta .. 125 nilotica (Pfr.) Burtoa .......... 189 nitens, Gmel. (Helix)............ 113 nitida, Drap. (Helix).......... 114 nitidissimus, Bgt. (Meladomus) .. 260 Nodularia, Conrad ........... 272 noitei, Bttg. Leucoch. ......... 178 nonotiensis, M. \& P. Ennea...... 81 noticola, Bs. Hydrocena ........ 269 novemlamellaris, Bnp. Endod. .. 128 novenaria, Anton (Achatina) .... 210 nuptialis (M. \& P.) Ena ....... nyassana, Bgt. (Corbicula) ..... 280

Obeliscus, Beck, see Euonyma

obesa, Gibb. Ennea .......... 282

obesa, Pfr. var. ............. 205

obliqua, von Mts. var......... 190

obliquus, Krs. (Ancylus) ....... 240

oblonga, von Mts. var......... 190

oblongus, Bttg. Leucoch. ..... 178

oblongus, Küst. va1. ......... 227

obovata, Pfr. Ennea ........... 81

obscurus, Küist. var. . ........ 22!

occidentalis, Mouss. Ampullaria.. 257

ochracea (M. \& P.) 'Iropidophora 256

oconnori, Prest. Natalina....... 96

octona (Brug.) Subulina ....... 210

oedigyra, M. \& P. Achatina ...... 197

oliphantensis, Crvn. Corbicula .. 279

olivacea, Irrs. var............. 277

olivaceus (Sow.) Lanistes ...... 258

omicronaria, M. \& P. (Pupa) .... 181

omphalion, Bs. (Helix) ......... 93

Oncaa, Gistel ............... 190

ONCHIDELLA, Gray........... 225

ONCHIDIID E, Gray ........ 224

Onchiniosi, Buch. ............. 224

Oncidiella, see Onchidella

Oncidium, see Onchidium

Oopeita, Mörch .............. 124

OOPELTIN $A$, Gkill............... 124

Opeas, Albers................ 211

opposita (Mouss.) Ena .......... 172

oppugnans, M. \& P. Ennea ...... 82

orcula (Bs.) Pupisoma .......... 159

ordinaria, M. \& P. Trach. ..... 141

ordinarius, M. \& P. Melampus .. 228

oreina, M. \& P. Trach. ....... 141

orientalis, Lam. (Cyrena) . . . . . . . 279

orophila, Morel. var. ........ 234

Orala, Schum. ............. 160

ottonis, Pfr. (Helix) ......... 116

ovampicum, Ancey, Pisidium .... 281

ovampoensis, M. \& P. (Pupa) .... 179

ovampoensis (M. \& P.), Cæcil .... 207

ovata, da Costa (Achatina) ..... 195

ovata (Krs.) Assemania.......... 268

ovata, Küst. var. ........... 228

ovoidea, Bgt. (Physa) ........ 250

ovularis, Kurr (Pupa) ........ 185

ovum, Ptrs. Lanistes........... 258

Pachnodos, Albers. .......... 164

pallescens, Ckll. Urocyclus . . . . . 123

pallida, Gibb.var. .......... 123

Paldoestrina, d'Orb. .......... 265

PALUDESTRINIDE, B. B. Woodw. 265

paludicola (Bs.) Phortion........ 130

Paludina, Lam. ................ 260

Paludinida, Auctt. ........... 260

Paludomus. Swains. ........... 261

pamphorodon (Bs.) Jaminia .... 187 
PAGE

panthera (Fér.) Achatina ..... 197

P'arachatina, Bgt. ............ 190

parallela, M. \& P. Ennea ..... 82

parasitica (Parr.) Eupera........ 280

parietalis (Mouss.) Isidora ...... 246

parma, Brard (Limacella)....... 119

Parmarion, Fischer .......... 123

parthenia, M. \& P. Achatina .... 198

parvispirum, Pfr. (Cyclostoma) .. 287

parvulus, Nuttall, Melampus .... 228

passargei, von MIts. Achatina .... 199

passargei, von Mts. Vivipara .... 260

patentissima, Mke. Succinea .... 222

patera, M. . P. Trach. ....... $1 \pm 1$

Patula, Held ............... 98

paula, M. \& P. (Trach.) ....... 138

PECTINIBRANCHIA, Cuvier.... 250

Pedipes, Fér. ............. 230

Pelecypoda, Goldf. = Submytilacea Pella, Alb., see Phortion.

pellicula (Fér.) Peltatus ....... 106 pellucens (Mke.) Auriculastra.. 231, 285

PELTATIN A, G.- Aust. ........ 104

Peltatus. G.-Aust. ........... 101 penestes, M. \& P. Achatina ... 199

pentheri, Stur. Ennea ......... 82

pentheri (Stur.) Eni ........ 172

pentheri, Stur. (Pupa) ......... 184

pentheri, Stur. Achatina ........ 199

pentheri, Stur. var. ......... 272

pentodon, Morel. Kinnea ........ 82

pepita, King, Marinula ......... 230

pereximia (N. \& P.) Jaminia .... 187

perfida, Bnp. Endodonta ........ 128

periploca, M. \& P. Ennea ..... 82

perissodonta, Stur. Ennea ..... 82

perlevis, Prest. Zingis ......... 99

permeata, M. \& P. Irach. ...... 141

peroni, Cuv. Onchidium ........ 224

Peronia, de Blainville ......... 224

perplexa (Bnp.) Jaminia .......... 183

perplicata (Bs.) Trach. ....... 141

perspicua, M. \& P. Ennea....... 83

perspicuæformis, Stur. Enrea.... 83

petersi (Pfr.) Ena ............ 173

petersi, von Mts. (Achatina)...... 194

petersi (von Mts.) Veronicella .. 223

petersi, von Mits. Spatha ....... 275

pethericki, Bgt. (Burtoa) ....... 190

petrobia (Bs.) Trach. ......... 142

pfeifferi (Kr's.) Ennea ......... 83

pfeifferi, Krs. Planorbis ....... 237

phædimus (M. \& P.) Kerkophorus 110

Phasis, Alb. .............. 129

Prontion, Preston .......... 129

phragma, M. \& P. Ennea........ 83

Physa, Drap., see Isidora

Physopsis, Krs. ..............

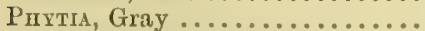

phytostylus (Bs.) Peltatus .....

picturata, Morel. Ena ......... pietersburgensis (Prest.) Euonyma 216 pinguis (Krs.) Trach. ......... 142

Pintoa, Bgt. ............. 190

pisana, Müll. Helix ......... 162

piscinalis, M. \& P. Succinea .... 222

Prsidiux, C. Pfi. ............. 281

pisolina, Gould (Nanina) ....... 283

Pisum, Gray ..............280, 281

placenta, M. \& P. 'Trochomorpha 103

planodiscus, M. \& P. Segmentina 239

Planonbis, Geoffroy .......... 235

planti, Pfr. Ennea .......... 83

planti (Pfr.) Helicarion ....... 100

planti (Pfr.) Trach. ........... 142

planti, Pfr, var.............. 189

planti, Pfr. Succinea.......... 222

platti, Pfr. (Helix) ........... 142

platyacme, M. \& P. Euonyma.... 216

PLATYPODA, Semper ....... 250

plicicutis, Bs. (Cyclophorus) .... 252

plurilirata, Fulton, Tropidophora 256

poppigi (Mke.) Kerkophorus .... 110

Polita, Held ............... 112

polita, M. \& P. Ennea .......... 84

Polygyra, say .............. 131

polypunctata, Cllge. Oopelta .... 126

Pomatia, Leach .............. 160

pomatia, Müll. (Helix) ......... 153

POMA'TIID E, B. B. Woodw. .... 252

pondoensis, G.-Aust. Microkerkus 111

ponsonbyana (Morel.) Jaminia .. 187

ponsonbyi, Fult. Dorcasia ..... 152

ponsonbyi (Cllge.) Milax ........ 121

ponsonbyi, Morel. Assemania.... 268

porphyrostoma (M. \& P.) Trig. .. 155

pottebergensis, Krs. (Pupa) ...... 185

premnodes, Stur. Ennea ........ 84

pretoriensis (M. \& P.) Jaminia .. 183

pretoriensis, M. \& P. (Helix) .... 117

princeps, Lea, Melanopsis........ 287

prionacis (Bs.) Trach. .......... 143

PRORHIPIDOGLOSSOMORPHA,

Grobben ................ 62

pruizenensis, Conn. Euonyma .. 216

psammophila (Bttg.) Ena........ 173

psichion, M. \& P. (Pupa) ........ 285

ptychaxis (Smith) Ena.......... 165

pulchella (Mïll.) Vallonia........ 159

pulchella, M. \& P. Ennea........ 84

pulchella, Morel. (Alexia) ..... 232

pulcherrima, Ang. (Neritina) .... 271

pulligerus (Lin.) Theodoxis ...... 270

PULMONATA, Cuv. ......... 62

pumilio (M. \& P.) Andrarion .... 101

punctata (Anton) Ena ......... 173

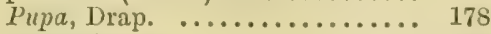

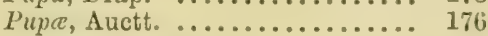

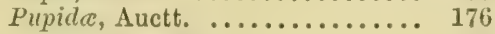

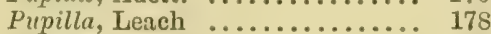

Pupisoura, Stol. ............. 158

Pupoides, Pfr. .............. 176 
PAGE

purcelli, Cllge. Apera ........ 63

purcelli (M. \& P.) Euonyma .... 216

jurpureus (Jonas) Lanistes ...... 259 jusilla (H. \& A. Ad.) Auriculastra 286 pusilla, Morel. (Fnnea)......... 80 pusilla (Parr.) Corbicula ... 277, \&c. pyenotrichn, M. \& P. I'Irach. .... 144 pygmeus, H. Ad. (Bulimulus) .... 169 pyrum, Poro (Physa) ....... 245

quantula (M. \& P.) Jaminia ... 183 queketti, M. \& P. Ennea ........ 84 quekettiana (M. \& P.) Natalina .. 96

linchisellus, Bgt.

16.1 radiata (Parr.) Corbicula ......... 279 radiolata (Morel.) Auriculastra .. 231 rariplicata (Bs.) Trach. ........ 144 rectilinearis, Sow. (Unio) ..... 272 regularis, M. \& P. Ennea........ 84 remota, Bs. (Helix) .......... 112 restitutoris, Humph. (Helix) ... 160 retisculpta (von Mts.) Sculptaria 132 reymondi, Bgt. (Bulimus)........ 190 rhabdota, M. \& P. Achatina .... 199 Rimachidina, Thiele .......... 164 Iihachis, Albers ............. 1fit Rirachiseluus, Bgt. . . . . . . . . . 164 Iihanada, Albers ............ 157 IHHIPIDOGLOSSA, Troschel .... 269

rhodesiana, Conn. Ennea....... 85 Rhodostoma, Swainson ......... 231 rhysodes, M. \& P. (Trach.) ..... 141 Rhytida, Albers ...........89, \&c. RHY'IDIDE, Pilsb. ......... 89 ridibunda, M. \& P. (Pupa) ..... 179 rinteleni, Bttg. var. .......... 132 rivularis (Krs.) Trach. .......... 145 rivularis, Phil. (Physa)......... 245 Rochebrunia, Bgt............ 253 rogersi, M. \& P. Ennea ....... 85 rosaceus (Mïll.) Trigonephrus .. 155 rosenbergi, Prest. Zingis ....... 100 roseri, Krs. (Helix)............. 147 rotula, M. \& P. (Trach.) ........ 128 rotundata, Mouss. var......... 150 rufofilosus, M. \& P. var. ........ 106 Irumina, Iisso ............ 285 rippelli, Dkr. Planorbis ........ 237 russofulgens (M. \& P.) Helicarion 101 rutilans, M. \& P. Trach. ....... 146

sabuletorum (Bs.) Trach. ...... 146 salinarum, Morel. Planorbis .... 238 sambesiensis, Stur. Vivipara .... 260 sarcodes (Pfr.) Tropidophora .... 25ั6 sarissa, Pilsb.var............. 218 saulcyi, Bgt. (Corbicula) ....... 279 saundersæ, Conn. Curvella ...... 209 Savignyi, Semp. Onchidium..... 225 saxicola, Ckll. Veronicella ..... 224 scrvola, M. \& P. Achatina ..... 139 scalaris, Dkr. (Bulinus) ....... 2.46 scalata, Merian (Physa) ....... 215 schackoi, Jick. Isidora ......... 217 schrerfire (Pfr.) Natalina ........ 96 schencki, von Mts. Achatina .... 200 schinziana, Mouss. Achatina .... 200 schmidti, Dkr. (Bulinus) ....... 216 schrencli, see schencki schultzei, Bttg. Ena ........... 17. schultzei, Srth. (Ceratoconcha) .. 282 schweinfurthi, von Mts. var. .... 190 scolopendra, M. \& P. Trach. .... 146 scrobiculata, M. \& P. Ennea ... 85 Sculptaria, Pfr. ............ 131 sculpturata (Gray) Sculptaria .... 132 sebasmia, Bgt. (Burtoa) ....... 190 sectilis, Fér. (Helix) .......... 281 Segmentina, Fleming ......... 238 sejuncta, Stur. (Ennea)......... 78 semiaratus, Conn. Melampus .... 228 semidecussata, Mke. Achatina .. 200 semigranosa, Pfr. (Achatina) .... 194 semiplicata, Morel. (Physa) ..... 216 semisulcatus, Mouss. Melampus .. 229 senegalensis (Morel.) Leucoch. .. 177 separata, Stur. Ennea ......... 86 sericina, Jick. Isidora ......... 247 Serpora, Bgt. ............... 190 sesamorum, Bgt. (Pachnodus) .... 170 Sheldonia, Ancey ............ 99 Sidula, Gray .............. 231 sigurensis, G.-Aust. Kaliella .... 117 siliqua, Conn. Euonyma ....... 217 similaris (Fér.) Eulota ......... 156 simillima, Stur. var.......... 77 simplex, Smith, Achatina ..... 201 simplex, M. \& P. Trach. ........ 147 sinensis, Nev. Assemania ........ 269 sinica, Nev. Assemania......... 269 sinistrorsa, Crvn. (Pupa) ....... 184 sinuosa, M. \& P. Curvella ..... 209 smithi, Crvn. Achatina......... 201 socratica, M. \& P. Ennea........ 86 solatus, Bs. (Bulimus) ......... 173 solidus, Smith, Lanistes ....... 257 sollers (M. \& P.) Phortion ..... 130 somersetensis (M. \& P.) Trach. .. 147 souverbianus (Montr.) Theodoxis 270 spadicea (Mlke.) Ena ........... 174 SPAтнA, Lea .............. 275 spathuliformis, Bgt. Spatha ..... 276 spekei, Bgt. (Bulimus) .......... 171 Sphzrium, Scopoli ........... 280 Sphalerostoma, Girard .......... 219 spilogramma (von MIts.) Ena ... 175 spiracea, Parr. (Physa)......... 245 spissicosta, M. \& P. Trach. .... 147 spumosa, Lowe (Helix) ........ 160 squalida, Zglr. (Helix) ......... 156 stabilis, Pollon. (Arion) ....... 126 
standeri, Conn. Euonyma $\ldots \ldots . \quad \begin{array}{rr}\text { P.IGE } \\ 217\end{array}$ stauroma, M. \& P. (Ennea) ..... 69 stenochorias, M. \& P. var. ..... 210 Stenogyra, Shutt..........211, ic. STENOGYRINE, Fischer ...... 207 sticta (von Mts.) Ena ......... 175 stimpsoni, Pfr. (Helix) ........ 157 stoaphora, Bs. (Pupa) ......... 1s3 straminea, Bnp. Curvella....... 209 STREPTAXID $\mathrm{E}$, Gray ....... 64 STREPTAXIN E, G.-Aust. .... 64 Streptaxis, Gray .......... 64 STREPTONEURA, Spengel .... 250 Streptostele, Dohrn ......... 89 striata, Krs. Succinea ........ 22. striatus, Pse. Melampus ....... 229 strigile (M. \& P.) Opeas ....... 212 strobilodes, M. \& P. (Helix) ...... 148 Strombus, Lin. ............. 263 sturmiana, Pfr. (Helix) .......... 95 STYLOMIATOPHORA, A. Schmidt 62 subcapense, Brt. Sphærium ...... 280 subcornea, Prest. (Helicarion) .... 109 subcylindrica, Prest. Achatina .. 201 subfuscus, Drap. (Limax) ....... 126 sublineare, Bttg. Opeas ....... 213 SUBMYTILACEA, de Blainville 271 subovata, da Costa (Achatina) .... 195 subradiata, Bttg. var. ........ 163 subteres, Bttg. Ena .......... 175 subtruncatula, Bttg. Limnæa .... 234 SubUlina, Beck. ........... 210 Siccinea, Drap. ............... 219 succinea, Bnp. Curvella ....... 209 SUCCINEID E, Tryon .......... 219 sulcata, Lam. (Cyclostoma) ..... 253 sulculosus, von Mts. Melampus .. 229 sydneyensis, Cox (Helix) ....... 113 sylesi (M. \& P.) Jaminia ........ 18t sylvaticus, Morel. (Limax) ...... 119 sylvia, M. \& l'. Ennea ......... 86 symmetricus (Crvn.) Microlierkus 111

tabula, Sow. (Anodon) .......... tabula, Chaper (Helicopsis)....... tabularis (M. \& P.) Jaminia .... TANIOGLOSSA, Troschel ..... tanganikana, Bgt. (Corbicula) .... tarachodes, Conn. Natalina ...... tasmanicus, Tate (Limax) ..... Tellina, Lin. .............. teres, Pfr. Truncatella .......... teretiuscula, M. \& P. Trach. .... terveriana, IVebb, Cochlicella .... Testacelua, Cuv. ............ TESTACELLID E, Gray......... tetrodus (Bttg.) Jaminia ........ Thipsiella, Gude ............ tharfieldensis, M. \& P. Ennea.... thaumasta, M. \& P. (Pupa) ..... Theba, Leach in Risso ......... thelodonta, M. \& P. Ennea ...... Tueodoxis, de Montfort ........ thermarum, M. \& P. Zingis...... thiarella, Lam. Melania ........ Trara, Bolten ............... tollini (Alb.) Trach. ............ Tonicmin, Bs............... tomlini, Bnp. var. ............ tonganum, Q. \& G. (Onchidium).. Tiscurcrstis, pilsb.............. transiens, Stur. Ennea........ 87 translucens, King (Helix) ....... 156 translucidum, Sow. (Cyclostoma) 251 transvaalensis (Crvn.) Helicarion 101 transvaalensis (M. \& P.) Ena .... 17i; transvaalensis, Smith, Achatina.. 201 transvaalensis, Crvn. Ancylus.... 241 transvaalensis (M. \& P.) Trop. .. 256 trapeziformis, Furt. var. ....... 25s trapezoideus, Bttg. var. ....... 240 trichostiroma (M. \& P.) Trach. .. 14s triglochis, M.\& P. Ennea ..... 87 Trigonephrus, Pilsb. ......... 153 trilamellaris, M. \& P. Endodonta 12s trimeni (M. \& P.) Natalina ...... 97 tristis (Morel.) Paludestrina ... 26.5 tristis, Jay (Bulimus) . . . . . . 250 triviu, Bttg. var. ........... 150 trochlea, Pfr. (Achatina) ........ 210 Trochomorpha, Alber's........ 103 Trochonanina, Mouss. ........ 10.2 TROCHONANININE, CONn.... 101 Trochozonites, Pfeffer........ 103 tropica (Krs.) Isidora ......... 247 TropidophorA, Troschel ...... 2.52 trotterianus (Bs.) Peltatus ..... 107 truncata, Fér. (Physa) ........ 24. Truveatella, lisso .......... 266 TRUNCATELLID E, Gray...... 266 Truncatula, Leach........... 26t; truncatula (Müll.) Limnæa...... . 234 truncatus, Brug. (Bulimus) ..... 234 tuberculata (Miill.) Melania..... 261 tugelense (M. \& P.) Opeas ...... 213 tuguriolum, M. \& P. (Trach.) .... 14s 'Tulbaghinia, M. \& P. . . . . . . 15: Turbo, Lin. ............. turmalis (Morel.) Trach. ........ 119 turriculata, Morel. (Physa) ..... 246 turriformis (Krs.) Euonyma...... 217 tyttha, M. \& P. (Assiminea) ... 269

uitenhagense (Krs.) Phortion .... 130 umbilicata, Drap. (Pupa) ....... 2\$. umlaasiana, Smith, Assemania .. 269 umlaasianus, Küst. Limnæa .... 23.j umlaasianus (Krs.) Melampus .. 229 unicolor (Oliv.) Vivipara ........ 260 unicornis, Conn. Euonyma ...... 218 Uxıo, Retz. ............. 274 UNIONID.E, Fleming ......... 


\begin{tabular}{|c|c|c|c|}
\hline & & & \\
\hline$\ldots \ldots$ & 19 & tus, \&c. & \\
\hline$\ldots \ldots$ & i23 & A, de Montfort . & \\
\hline Lus, Gray & 123 & VIVIPARID E, Gray & \\
\hline$\ldots \ldots \ldots$ & 21 & $\begin{array}{l}\text { vorticialis (Bs.) Trach. } . . . . . . . . ~ \\
\text { vulgaris, da Costa (Cochlea) .... }\end{array}$ & \\
\hline er ( & 272 & & \\
\hline & $2 \cdot 2$ & wahlbergi (Krs.) Ennea & \\
\hline tianus, Fér. (Limax) & 119 & (Bs.) Cyclophorus & \\
\hline ris, M. \& P. (Ennea) & 74 & (Krs.) Spatha .... & \\
\hline sso $\ldots \ldots \ldots \ldots$. & 15 & , Krs. (Physa) .... & \\
\hline (Crvn.) Helicarıon & 10 & If. \& P. Ennea .... & \\
\hline ea .. & 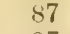 & ch. (Physa) .... & \\
\hline nea $\quad .$. & 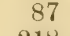 & var...... & \\
\hline$\ldots \ldots$ & 2 & $\ldots \ldots$ & \\
\hline$\cdots \cdots$ & 2( & nnea .. & \\
\hline $\begin{array}{c}\ldots \ldots \\
\ldots \ldots\end{array}$ & $16 \pm$ & Mill. & \\
\hline cus)...... & 127 & $a_{\ldots} . . . .$. & \\
\hline$\ldots \ldots$ & 26 & & \\
\hline p. (Bulimus) .... & 15 & s) $\ldots$ & \\
\hline Helix) & 1 & s, Walker, Aneylus .. & \\
\hline ...... & 27 & , Prest. Unio & \\
\hline & 97 & ssis, Petit (Melania) .. & \\
\hline$\cdot$ & 20 & Morel. (E) & \\
\hline ray ... & 2 & (Petit) Tr & \\
\hline o) $\ldots \ldots$ & 2 & $n$, Pfr. (C) & \\
\hline $1 s \quad \ldots \ldots$. & 2 & Cless.) Isi & \\
\hline$\ldots \ldots \ldots$ & 21 & • & \\
\hline o) $\ldots \ldots$ & 9 & - & \\
\hline$\cdots \cdots \cdots$ & & - & \\
\hline . Woodw... & & - & \\
\hline$\ldots \ldots \ldots$ & & $a \ldots$. & \\
\hline & & - & \\
\hline$\cdots \cdots \cdots$ & 2 & - & \\
\hline ncylus .... & $24:$ & Kerkophorus & 11 \\
\hline 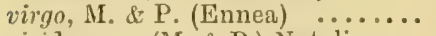 & S & see Polita, \&c. & \\
\hline Natalina .. & & & \\
\hline sophorus .. & & b. & \\
\hline & & a. $\quad \ldots \ldots \ldots \ldots$ & \\
\hline$\ldots \ldots$ & 7 & sterl. & \\
\hline 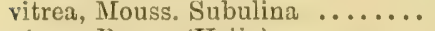 & & . & \\
\hline x) $\ldots \ldots \ldots$ & & & \\
\hline$\ldots \ldots \ldots$ & & & \\
\hline
\end{tabular}



PARTS OF THE ANNALS PREVIOUSLY ISSUED:-

Vol. I.-Part 1, 7/6; Part 2, 10/-: Pant 8, 5/-; oomplete 81 2s. 6 d.

Vol. 11.-Part 1, 2/6; Part $8,6 /-$; Part $8,1 /-$; Part $4,2 / 6$; Part 5, 1/-; Part 6, 2/6;

Part 7, 1/-; Part 8, 2\%6; l'art 9, 1-/;

Part 10,6/-: Vaxt 11. 2/6; Index, de, 1 /-; complete $218 \% .6 \%$.

VoI. III. Part 1. 2/-: Patt 3, 1/-; Part 3, 5/-;

Part 4, 2/6; Part 5, 5/-; Part 6, 6/-;

Part $7 ; 1 /-;$ Part $8,2 / 6$; Part 9, 1/-;

Index, Title, \&c., $1 /-\quad \cdots \quad \because \quad$ complete $£ 17 \mathrm{~s}, 0 d$.

Vol. IV. (containing Palæontological papers published

in conjunction with the Geological Survey).--

Part 1, 10/-; Part 2, 6/-; Part . 3, 4/-;

Part 4, 4/-; Pait 5, 2/-; Part 6, 4/-;

Part $7,12 / 6$; Fant 8. $7 /$ - . . complete $1298.6 d$.

Vol. V.-Part 1, 4/-; Part $2,7 / 6$; Part $3,2 /-$;

Part 4, $1 /-$; Part 5, $1 / 6$; Part 6, 4/6;

Part $7,2 / 6$; Part $8,4 /-$; Part $9,4 /-$;

Index, Title, de., $1 /=$. . . . complete $\$ 112 \mathrm{~s} .0 \mathrm{~d}$.

Vol. VI.-Part 1, 12/-; Part 2, 4/-; Part 3, 3/-;

Part 4, 27/-; Index, Title, \&c., $1 /$ o complete $£ 278.0 d$.

Vol. VII. (containing Palæontological papers published

in conjunction with the Geological Survey).-

Part $1,2 / 6 ; 2,12 / 6 ;$ Part $3,4 / 6$;

Paut 4, $7 /$; Part $5,5 /-$.

Vol, VIII.-Part 1, 40/-.

Vol. IX.-Part 1, 4/- ; Part 2; 5/-.

Vol. X.-Part 1, 2/6; Part 2, 2/-; Part 3, $1 / 6$.

Vol. XI.-Part 1, 3/-; Part $2,1 / 6$.

The Annals of the Soith African Miseum will be issued at irregular intervals; as matter for publication is available.

Copies may be obtained from-

MEssRs. WEST, NEWMAN \& Co.,

54, HatTon GaRDEN, London.

Mesirs. WILLIAM WESLEY \& SON,

28, Egsex Street, Strand, London.

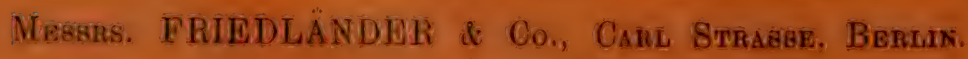

Or.

THE LibRarian, South Amgioan Muszum, Cape Town. 





